\title{
EMPREGO DE FORMULAÇÕES NÃO-CONVENCIONAIS DE ELEMENTOS FINITOS NA ANÁLISE LINEAR BIDIMENSIONAL DE SÓLIDOS COM MÚLTIPLAS FISSURAS
}

Dissertação apresentada à Escola de Engenharia de São Carlos, Universidade de São Paulo, como parte dos requisitos para a obtenção do título de Mestre em Engenharia de Estruturas.

Orientador: Prof. Tit. Sergio Persival Baroncini Proença

São Carlos 
AUTORIZO A REPRODUÇÃO E DIVULGAÇÃO TOTAL OU PARCIAL DESTE TRABALHO, POR QUALQUER MEIO CONVENCIONAL OU ELETRÔNICO, PARA FINS DE ESTUDO E PESQUISA, DESDE QUE CITADA A FONTE.

Ficha catalográfica preparada pela Seção de Tratamento da Informação do Serviço de Biblioteca - EESC/USP

Argôlo, Higor Sérgio Dantas de

A693e Emprego de formulações não-convencionais de elementos finitos na análise linear bidimensional de sólidos com múltiplas fissuras / Higor Sérgio Dantas de Argôlo ; orientador Sergio Persival Baroncini Proença. -- São Carlos, 2010.

Dissertação (Mestrado-Programa de Pós-Graduação e Área de Concentração em Engenharia de Estruturas) -- Escola de Engenharia de São Carlos da Universidade de São Paulo, 2010 .

1. Método dos elementos finitos. 2. Método dos elementos finitos - formulações não-convencionais. 3. Formulação híbrida-Trefftz de tensão. 4. Método dos elementos finitos generalizados. 5. Método de partição. 6. Fator de intensidade de tensão. I. Título. 
FOLHA DE JULGAMENTO

Candidato: Engenheiro HIGOR SÉRGIO DANTAS DE ARGÔLO.

Dissertação defendida e julgada em 24.09.2010 perante a Comissão Julgadora:

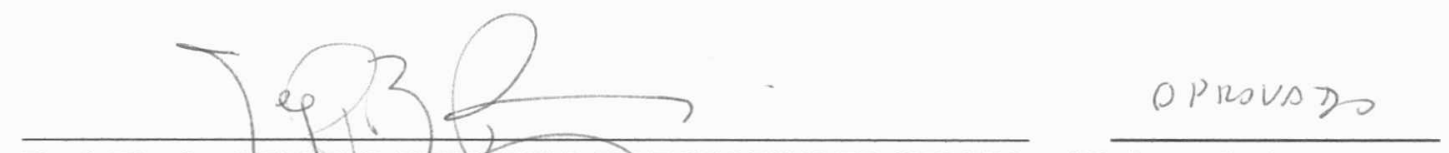

Prof. Titular SERGIO PERSIVAL BARONCINI PROENÇA - (Orientador)

(Escola de Engenharia de São Carlos/USP)

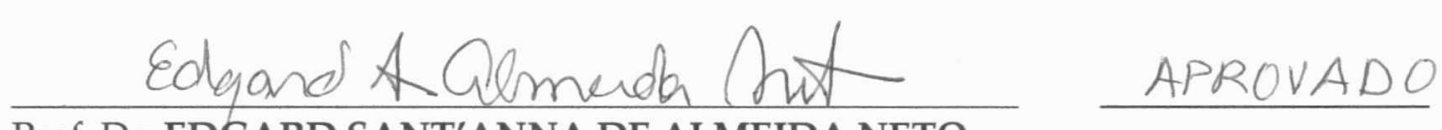

Prof. Dr. EDGARD SANT'ANNA DE ALMEIDA NETO (Escola Politécnica/USP)

Prof. Titular WALTER SAVASSI

(Escola de Engenharia de São Carlos/USP)
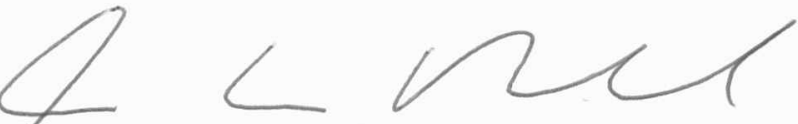

Prof. Associado MARCIO ANTONIO RAMALHO

Coordenador do Programa de Pós-Graduação em

Engenharia Civil (Engenharia de Estruturas)

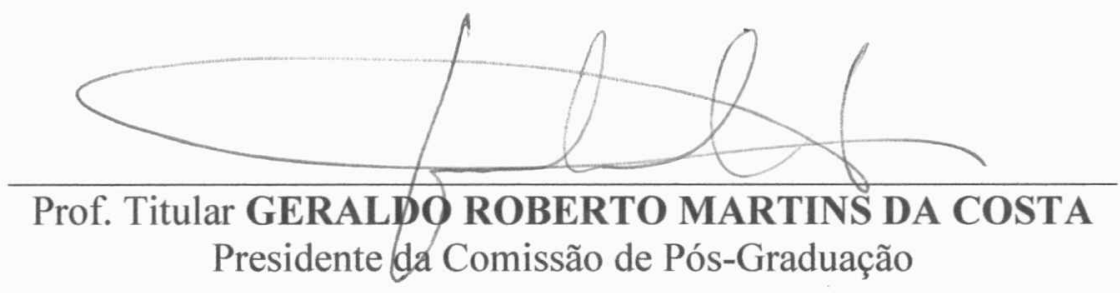



Dedico esse trabalho aos meus pais Sérgio e Silvânia, pois sem eles eu não teria a oportunidade de chegar até aqui. 


\section{AGRADECIMENTOS}

Aos meus pais Sérgio e Silvânia que sempre se empenharam em me dar uma boa educação.

Aos meus avós Edla, Sinval, Helena e Argôlo.

À Milena que apesar da distância esteve junto de mim nesses anos de trabalho.

Aos meus irmãos Herick e Hiago pela convivência durante todos esses anos.

Aos meus amigos de Aracaju.

Aos meus amigos da Universidade Federal de Sergipe, bem como os professores que me ajudaram a dar esse primeiro passo rumo à engenharia.

Aos amigos da USP de São Carlos que conviveram comigo durante esses anos de mestrado.

Aos professores e funcionários da Escola de Engenharia de São Carlos.

Ao meu orientador Sergio Proença que sempre esteve disposto a dividir seu conhecimento comigo.

Ao CNPq por ter financiado esta pesquisa. 
"Que os vossos esforços desafiem as impossibilidades, lembrai-vos de que as grandes coisas do homem foram conquistadas do que parecia impossível."

(Charles Chaplin) 


\section{RESUMO}

ARGÔLO, H. S. D. de (2010). Emprego de formulações não-convencionais de elementos finitos na análise linear bidimensional de sólidos com múltiplas fissuras. Dissertação de Mestrado, Escola de Engenharia de São Carlos, Universidade de São Paulo, São Carlos.

O trabalho trata da utilização de formulações não-convencionais de elementos finitos na obtenção de fatores de intensidade de tensão associados a múltiplas fissuras distribuídas num domínio bidimensional. A formulação do problema de múltiplas fissuras baseia-se numa abordagem de sobreposição proposta pelo Método da Partição ("Splitting Method"). Segundo essa abordagem a solução do problema pode ser encontrada a partir da sobreposição de três subproblemas combinados de tal forma que o fluxo de tensão resultante nas faces das fissuras seja nulo. O uso do Método dos Elementos Finitos (MEF) em sua forma convencional pode requerer um refinamento excessivo da rede nesse tipo de problema, aumentando o custo computacional da análise. Objetivando reduzir este custo, empregam-se duas formulações não-convencionais, de forma independente, num dos subproblemas, dito local: a formulação híbrido-Trefftz de tensão e o Método dos Elementos Finitos Generalizados (MEFG). Na formulação híbrido-Trefftz é adotado o recurso do enriquecimento seletivo mediante o refinop na aproximação dos campos de deslocamento no contorno do elemento. Já com relação ao MEFG, empregam-se funções polinomiais e a solução analítica da mecânica da fratura como funções enriquecedoras. Exemplos de simulação numérica são apresentados no sentido de comprovar que a utilização dessas formulações não-convencionais juntamente com o Método da Partição viabiliza a obtenção de resultados com boa aproximação com recurso a redes pouco refinadas, reduzindo significativamente o custo computacional de toda a análise.

Palavras-chave: Formulações não-convencionais de elementos finitos, Formulação híbridoTrefftz de tensão, Método dos Elementos Finitos Generalizados, Método da Partição, Fator de Intensidade de Tensão. 


\section{ABSTRACT}

ARGÔLO, H. S. D. de (2010). Use of non-conventional formulations of finite element method in the analysis of linear two-dimensional solids with multiple cracks. M.Sc. Dissertation, Escola de Engenharia de São Carlos, Universidade de São Paulo, São Carlos.

This paper treats with the use of non-conventional finite element formulations to obtain the stress intensity factor of multiple cracks located in a two-dimensional domain. The formulation of the multiple cracks problem is based on an overlapping approach suggested by the Splitting Method. Accordingly, the solution of the problem can be achieved by dividing the problem in three steps, combined so that the resulting stress flux is zero on the cracks face. The use of the Finite Element Method (FEM) in its conventional formulation requires a mesh refinement in this kind of problem, then increasing the computational cost. Aiming to reduce this cost, two non-conventional formulations are used independently to solve the local problem: the Hybrid-Trefftz stress formulation and the Generalized Finite Elements Method (GFEM). The Hybrid-Trefftz formulation is applied with selective enrichment using prefinement in the displacements field on the element boundaries. The GFEM employs polynomial functions and analytical solutions of the fracture mechanics as enrichment functions. Examples of numerical simulations are presented in order to show that nonconventional formulations and the Splitting Method can provide accurate results with coarse mesh, thus reducing the computational cost.

Keywords: Non-conventional formulations of finite element method, Hybrid-Trefftz stress formulation, Generalized Finite Elements Method, Splitting Method, stress intensity factor. 


\section{LISTA DE FIGURAS}

Figura 2.1 - Representação genérica de um sólido bidimensional. ................................ 6

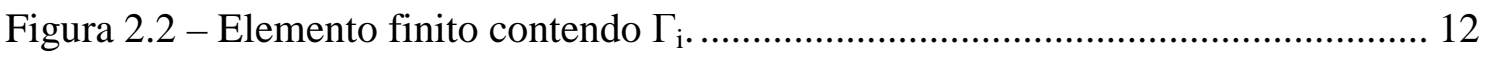

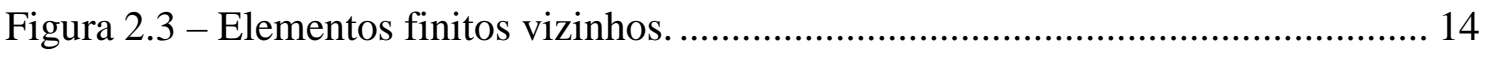

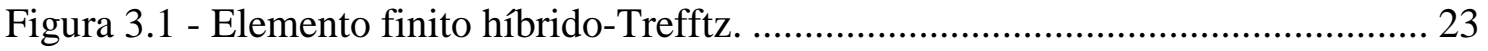

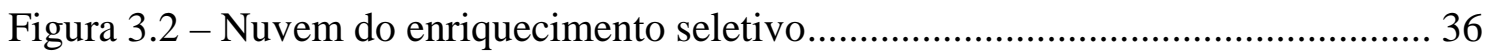

Figura 3.3 - Representação gráfica das funções da base hierárquica ............................. 38

Figura 3.4 - Representação gráfica das funções da base não-hierárquica ...................... 39

Figura 3.5 - Representação gráfica das funções da base trigonométrica....................... 40

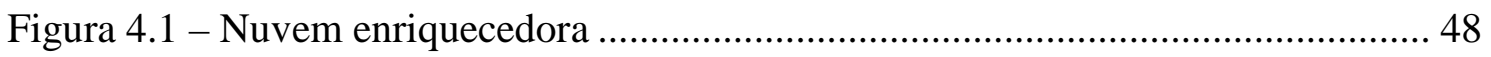

Figura 4.2 - Elemento finito em coordenadas cartesianas ......................................... 50

Figura 4.3 - Elemento finito em coordenadas adimensionais .................................... 51

Figura 4.4 - Elemento finito com os quatro nós enriquecidos ...................................... 56

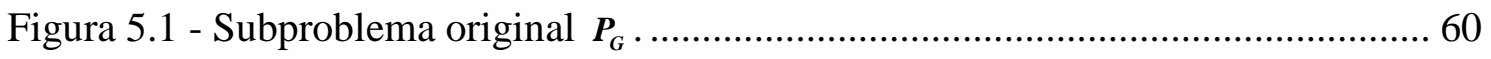

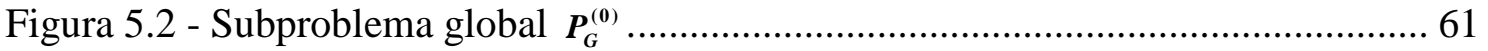

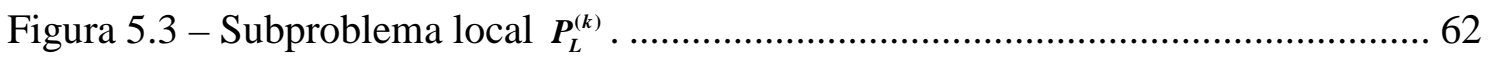

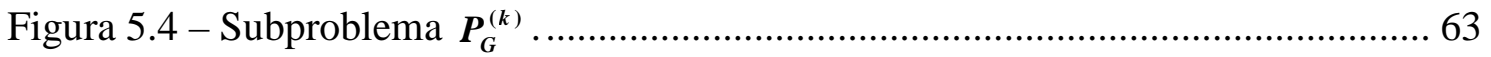

Figura 6.1 - Chapa com uma fissura e carregamento constante ................................... 69

Figura 6.2 - Modelo do $1^{\circ}$ problema utilizando a formulação híbrido-Trefftz .............. 70

Figura 6.3 - Rede do $1^{\circ}$ problema utilizando a formulação híbrido-Trefftz ................... 71

Figura 6.4 - Detalhe da região próximo à fissura .............................................................. 71

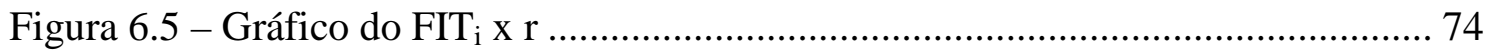

Figura 6.6 - Modelo do $1^{\circ}$ problema utilizando o MEFG ............................................ 75

Figura 6.7 - Rede do $1^{\mathrm{o}}$ problema utilizando o MEFG................................................ 75

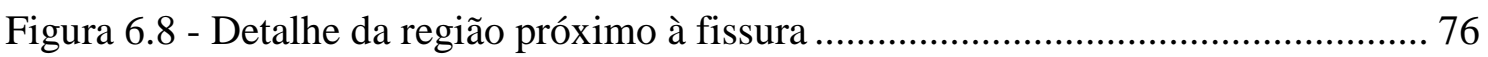

Figura 6.9 - Rede de elementos finitos híbrido-Trefftz do $2^{\circ}$ problema ........................ 78

Figura 6.10 - Detalhe da região interna ao contorno gama.......................................... 78

Figura 6.11 - Primeiro subproblema local. ............................................................... 79

Figura 6.12 - Modelo para o primeiro subproblema local .......................................... 79

Figura 6.13 - Curva de FIT $_{\mathrm{i}}$ x r para o primeiro subproblema local ............................. 80

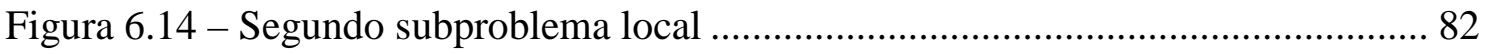




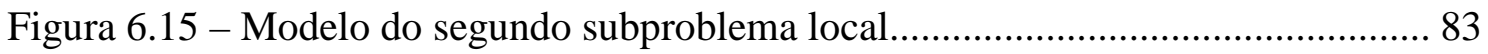

Figura 6.16 - Curva de FIT $_{\mathrm{i}}$ x r para o segundo subproblema local ............................... 83

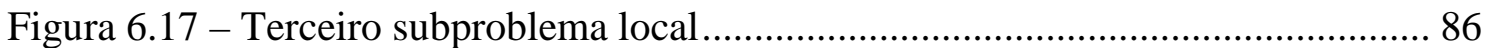

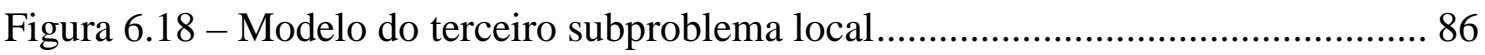

Figura 6.19 - Curva de FIT $_{\text {i }}$ x r para o terceiro subproblema local............................... 87

Figura 6.20 - Rede de elementos finitos do segundo problema utilizando o MEFG..... 90

Figura 6.21 - Detalhe da região interna ao contorno gama .......................................... 90

Figura 6.22 - Modelo para o primeiro subproblema local ............................................ 91

Figura 6.23 - Modelo para o segundo subproblema local ........................................... 92

Figura 6.24 - Modelo para o terceiro subproblema local .............................................. 93

Figura 6.25 - Chapa com duas fissuras e carregamento não - uniorme .......................... 94

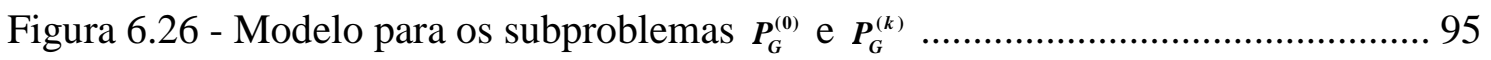

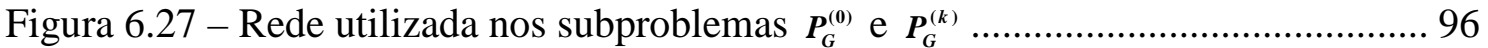

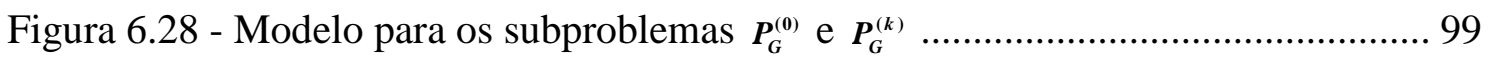

Figura 6.29 - Rede dos subproblemas $\mathrm{P}_{G}^{(0)}$ e $\mathrm{P}_{G}^{(k)}$ do $3^{\circ}$ problema................................ 99

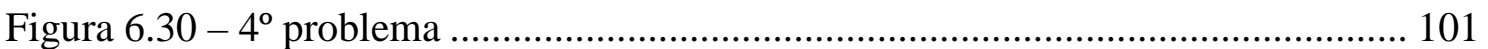

Figura 6.31 - Modelo dos subproblemas $\boldsymbol{P}_{G}^{(0)}$ e $\boldsymbol{P}_{G}^{(k)}$ do $4^{\mathrm{o}}$ problema .............................. 102

Figura 6.32 - Modelo do subproblema $\boldsymbol{P}_{L}^{(k)}$ do $4^{\mathrm{o}}$ problema...................................... 103

Figura 6.33 - Rede dos subproblemas $\boldsymbol{P}_{G}^{(0)}$ e $\boldsymbol{P}_{G}^{(k)}$ do $4^{\mathrm{o}}$ problema................................... 103

Figura 6.34 - Rede do subproblema $\boldsymbol{P}_{L}^{(k)}$ do $4^{\mathrm{o}}$ problema............................................ 104

Figura 6.35 - Detalhe do contorno gama da rede do subproblema $\boldsymbol{P}_{L}^{(k)}$ do $4^{\mathrm{o}}$ problema104

Figura 6.36 - Rede dos subproblemas $\boldsymbol{P}_{L}^{(k)}$ do $4^{\mathrm{o}}$ problema ....................................... 107

Figura 6.37 - Rede dos subproblemas $\boldsymbol{P}_{G}^{(0)}$ e $\boldsymbol{P}_{G}^{(k)}$ do $4^{\mathrm{o}}$ problema .............................. 108 


\section{LISTA DE TABELAS}

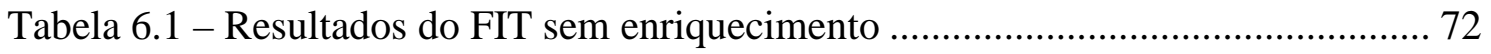

Tabela 6.2 - Resultados do FIT com enriquecimento hierárquico ................................ 72

Tabela 6.3 - Resultados do FIT com enriquecimento não-hierárquico......................... 72

Tabela 6.4 - Resultados do FIT com enriquecimento trigonométrico ........................... 73

Tabela 6.5 - Resultados do FIT utilizando o MEFG ..................................................... 76

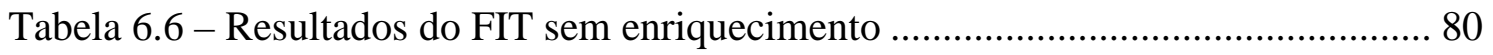

Tabela 6.7 - Resultados do FIT com enriquecimento hierárquico................................ 81

Tabela 6.8 - Resultados do FIT com enriquecimento não-hierárquico......................... 81

Tabela 6.9 - Resultados do FIT com enriquecimento trigonométrico ........................... 81

Tabela 6.10 - Resultados do FIT sem enriquecimento ............................................. 84

Tabela 6.11 - Resultados do FIT com enriquecimento hierárquico ............................... 84

Tabela 6.12 - Resultados do FIT com enriquecimento não-hierárquico ......................... 84

Tabela 6.13 - Resultados do FIT com enriquecimento trigonométrico ........................... 85

Tabela 6.14 - Resultados do FIT sem enriquecimento .................................................. 87

Tabela 6.15 - Resultados do FIT com enriquecimento hierárquico .............................. 88

Tabela 6.16 - Resultados do FIT com enriquecimento não-hierárquico ........................ 88

Tabela 6.17 - Resultados do FIT com enriquecimento trigonométrico .......................... 88

Tabela 6.18 - Resultados do FIT com MEFG …........................................................... 91

Tabela 6.19 - Resultados do FIT com MEFG ................................................................ 92

Tabela 6.20 - Resultados do FIT com MEFG ……........................................................ 93

Tabela 6.21 - Resultados do $3^{\circ}$ problema sem enriquecimento ................................... 97

Tabela 6.22 - Resultados do $3^{\circ}$ problema com enriquecimento hierárquico ................. 97

Tabela 6.23 - Resultados do $3^{\circ}$ problema com enriquecimento não-hierárquico ........... 97

Tabela 6.24 - Resultados do $3^{\circ}$ problema com enriquecimento trigonométrico............. 98

Tabela 6.25 - Resultados do $3^{\circ}$ problema com o MEFG …......................................... 100

Tabela 6.26 - Resultados do $4^{\mathrm{o}}$ problema sem enriquecimento.................................... 105

Tabela 6.27 - Resultados do $4^{\circ}$ problema com enriquecimento hierárquico ................. 105

Tabela 6.28 - Resultados do $4^{\circ}$ problema com enriquecimento não-hierárquico .......... 105

Tabela 6.29 - Resultados do $4^{\circ}$ problema com enriquecimento trigonométrico............ 106

Tabela 6.30 - Resultados do $4^{\circ}$ problema com o MEFG …........................................ 108 


\section{LISTA DE ABREVIATURAS E SIGLAS}

PVC - Problema de valor de contorno.

MEF - Método dos Elementos Finitos.

MSM - Método Sem Malha.

MEFG - Método dos Elementos Finitos Generalizados.

MP - Método da Partição.

FIT - Fator de intensidade de tensão.

HT-D - Hybrid - Trefftz displacement.

LFE - Large finite elements.

HTS - Hybrid - Trefftz stress.

MEC - Método dos Elementos de Contorno.

EPT - Estado plano de tensões.

EPD - Estado Plano de Deformações.

PU - Partição da unidade.

MEFPU - Método dos Elementos Finitos Partição da Unidade. 


\section{LISTA DE SÍMBOLOS}

$\Omega$ - Domínio do sólido ou da estrutura.

b - Vetor de forças volumétricas.

$\Gamma$ - Contorno do sólido ou da estrutura.

$\Gamma_{\mathrm{u}}$ - Fronteira cinemática do contorno do sólido ou da estrutura.

$\Gamma_{\sigma}$ - Fronteira estática do contorno do sólido ou da estrutura.

$\overline{\mathbf{t}}$ - Vetor de forças de superfície prescritas.

$\overline{\mathbf{u}}$ - Vetor de deslocamentos prescritos.

L - Matriz formada por operadores diferenciais.

$\boldsymbol{\varepsilon}$ - Tensor de deformações.

u - Vetor de deslocamentos.

f - Tensor constitutivo de flexibilidade.

$\boldsymbol{\sigma}-$ Tensor de tensões.

D - Tensor constitutivo de rigidez.

I - Matriz formada pelas componentes de um vetor unitário normal a $\Gamma_{\sigma}$.

$\partial / \partial \mathrm{x}$ - Derivada parcial em relação a x.

$\partial / \partial y$ - Derivada parcial em relação a y.

$\sigma_{\mathrm{x}}$ - Componente normal do tensor de tensões na direção x.

$\sigma_{\mathrm{y}}$ - Componente normal do tensor de tensões na direção y.

$\tau_{\mathrm{xy}}$ - Componente normal do tensor de tensões.

$b_{x}$ - Componente do vetor de forças volumétricas na direção $x$.

$b_{y}$ - Componente do vetor de forças volumétricas na direção y.

$\varepsilon_{\mathrm{x}}$ - Componente de deformação linear específica do tensor de deformações na direção x.

$\varepsilon_{\mathrm{y}}$ - Componente de deformação linear específica do tensor de deformações na direção y.

$\gamma_{\mathrm{xy}}$ - Componente referente à distorção angular do tensor de deformações.

$\mathrm{u}_{\mathrm{x}}$ - Componente do vetor de deslocamento na direção $\mathrm{x}$.

$\mathrm{u}_{\mathrm{y}}$ - Componente do vetor de deslocamento na direção y.

E - Módulo de Young.

$v$ - Coeficiente de Poisson.

$\mathrm{n}_{\mathrm{x}}$ - Componentes de um vetor unitário normal a $\Gamma_{\sigma}$.

$\mathrm{n}_{\mathrm{y}}$ - Componentes de um vetor unitário normal a $\Gamma_{\sigma}$. 
$\overline{\mathrm{t}}_{\mathrm{x}}$ - Componente do vetor de forças de superfície prescritas na direção $\mathrm{x}$.

$\overline{\mathrm{t}}_{\mathrm{y}}$ - Componente do vetor de forças de superfície prescritas na direção y.

$\overline{\mathrm{u}}_{\mathrm{x}}-$ Componente do vetor de deslocamentos prescritos na direção $\mathrm{x}$.

$\overline{\mathrm{u}}_{\mathrm{y}}$ - Componente do vetor de deslocamentos prescritos na direção y.

$\mathbf{M}_{\boldsymbol{\Omega}}, \mathbf{P}_{\boldsymbol{\Omega}}, \mathbf{V}_{\Gamma_{\boldsymbol{\sigma}}}$ - Funções peso.

$\mathbf{m}, \mathbf{p}, \mathbf{v}$ - Campos aproximados quaisquer.

$\delta \mathrm{m}_{\Omega}, \delta \mathrm{p}_{\Omega}, \delta \mathrm{v}_{\Gamma_{\sigma}}$ - Parâmetros da aproximação de $\mathbf{m}, \mathbf{p}, \mathbf{v}$, respectivamente.

$\mathbf{u}_{\Gamma}$ - Vetor de deslocamentos no contorno estático do elemento.

$\mathbf{S}_{\boldsymbol{\Omega}}$ - Matriz que coleta as funções de aproximação da combinação linear de $\boldsymbol{\sigma}$.

$\mathbf{U}_{\boldsymbol{\Omega}}$ - Matriz que coleta as funções de aproximação da combinação linear de $\mathbf{u}$.

$\mathbf{U}_{\Gamma_{\boldsymbol{\sigma}}}$ - Matriz que coleta as funções de aproximação da combinação linear de $\mathbf{u}_{\Gamma}$.

$\mathbf{X}$ - Vetor de parâmetros da combinação linear de $\boldsymbol{\sigma}$.

$\mathbf{q}_{\Omega}$ - Vetor de parâmetros da combinação linear de $\mathbf{u}$.

$\mathbf{q}_{\Gamma_{\boldsymbol{\sigma}}}$ - Vetor de parâmetros da combinação linear de $\mathbf{u}_{\Gamma}$.

$\boldsymbol{e}_{\Gamma_{\mathrm{u}}}, \mathbf{Q}_{\Omega}, \mathbf{Q}_{\Gamma_{\boldsymbol{\sigma}}}-$ Vetores do sistema linear das formulações não -convencionais.

$\mathbf{F}, \mathbf{G}_{\boldsymbol{\Omega}}, \mathbf{G}_{\Gamma_{\boldsymbol{\sigma}}}$ - Matrizes do sistema linear das formulações não-convencionais.

$\Gamma_{\mathrm{i}}$ - Fronteira comum a dois elementos.

$\mathbf{t}_{\Gamma_{\mathbf{u}}}$ - Vetor de forças do contorno cinemático.

$\mathbf{u}_{\Gamma_{\mathrm{i}}}$ - Vetor de deslocamentos em $\Gamma_{\mathrm{i}}$.

$\mathbf{t}_{\Gamma_{\mathrm{i}}}$ - Vetor de forças em $\Gamma_{\mathrm{i}}$

$\mathbf{W}_{\Gamma_{\mathrm{u}}}, \mathbf{U}_{\Gamma_{\mathrm{i}}}, \mathbf{W}_{\Gamma_{\mathrm{i}}}$ - Matrizes da combinação linear de $\mathbf{t}_{\Gamma_{\mathrm{u}}}, \mathbf{u}_{\Gamma_{\mathrm{i}}}$ e $\mathbf{t}_{\Gamma_{\mathrm{i}}}$, respectivamente.

$\mathbf{z}_{\Gamma_{u}}, \mathbf{q}_{\Gamma_{\mathrm{i}}}, \mathbf{z}_{\Gamma_{\mathrm{i}}}-$ Vetores da combinação linear de $\mathbf{t}_{\Gamma_{\mathbf{u}}}, \mathbf{u}_{\Gamma_{\Gamma_{i}}}$ e $\mathbf{t}_{\Gamma_{\mathrm{i}}}$, respectivamente.

$\mathbf{G}_{\Gamma_{\mathbf{i}}}$ - Matrizes do sistema linear das formulações não-convencionais numa rede com mais de um elemento.

$\mathbf{Q}_{\Gamma_{\mathbf{i}}}$ - Vetores do sistema linear das formulações não -convencionais numa rede com mais de um elemento.

$\mathbf{U}_{\Gamma_{\mathbf{i}}}$ - Matriz que coleta as funções de aproximação da combinação linear de $\mathbf{u}_{\Gamma_{\mathbf{i}}}$. 
$\mathbf{u}_{\Gamma_{\mathbf{i}}}$ - Vetor de deslocamentos em $\Gamma_{\mathrm{i}}$.

$\mathbf{t}$ - Vetor de forças de contorno.

$\mathbf{o}_{\Gamma_{\mathrm{i}}}$ - Vetor de parâmetros da combinação linear de $\mathbf{t}$.

$\mathbf{C}_{\Gamma_{\mathrm{i}}}^{+}, \mathbf{C}_{\Gamma_{\mathrm{i}}}^{-}-$Matrizes de $\mathbf{Q}_{\Gamma_{\mathrm{i}}}$

$\boldsymbol{\delta} \mathbf{t}$ - Forças virtuais em $\Gamma_{\mathrm{i}}$.

$\boldsymbol{\delta} \mathbf{p}_{\Gamma_{\mathbf{i}}}$ - Parâmetros da aproximação de $\boldsymbol{\delta} \mathbf{t}$.

$\delta \mathbf{u}_{\Gamma_{\mathrm{i}}}$ - Deslocamentos virtuais em $\Gamma_{\mathrm{i}}$.

$\delta \mathbf{q}_{\Gamma_{\mathbf{i}}}$ - Parâmetros da aproximação de $\delta \mathbf{u}_{\Gamma_{\mathbf{i}}}$.

$\phi_{1}, \phi_{2}$ - Funções de forma lineares.

$\mathfrak{R}($.$) - Operador matricial.$

$\hat{\mathbf{u}}$ - Vetor de deslocamentos referente a solução particular.

$\mathbf{u}_{\mathbf{h}}$ - Vetor de deslocamentos referente a solução homogênea.

$\hat{\mathbf{u}}_{\mathbf{x}}$ - Componente de $\hat{\mathbf{u}}$ na direção $\mathrm{x}$.

$\hat{\mathbf{u}}_{\mathbf{y}}$ - Componente de $\hat{\mathbf{u}}$ na direção y.

$\mathbf{N}$ - Matriz que armazena as funções aproximadoras para a combinação linear de $\mathbf{u}_{\mathbf{h}}$.

c - Vetor de parâmetros para a combinação linear de $\mathbf{u}_{\mathbf{h}}$.

$\mathbf{N}_{\mathbf{i}}$ - Vetor de funções aproximadoras que compõe $\mathbf{N}$.

$c_{i}$ - Vetor de parâmetros que compõe c.

$\mathrm{N}_{\mathrm{ix}}, \mathrm{N}_{\mathrm{iy}}$ - Componentes de $\mathbf{N}_{\mathrm{i}}$.

Z - Número complexo.

$\overline{\mathrm{Z}}$ - Número complexo conjugado.

$\mathrm{F}(\mathrm{z}), \mathrm{G}(\mathrm{z})$ - Pares de funções arbitrárias.

i - Unidade imaginária de um número complexo.

$\mathbf{N}_{\mathbf{A k}}$ - Sequência 1 de vetores da solução homogênea.

$\mathbf{N}_{\mathbf{B k}}$ - Sequência 2 de vetores da solução homogênea.

$\mathbf{N}_{\mathbf{C k}}$ - Sequência 3 de vetores da solução homogênea.

$\mathbf{N}_{\mathbf{D k}}$ - Sequência 4 de vetores da solução homogênea. 
$A_{k}$ - Funções que definem a sequência $\mathbf{N}_{\mathbf{A k}}$.

$\mathrm{B}_{\mathrm{k}}$ - Funções que definem a sequência $\mathbf{N}_{\mathbf{B k}}$.

$\mathrm{C}_{\mathrm{k}}$ - Funções que definem a sequência $\mathbf{N}_{\mathbf{C k}}$.

$D_{k}$ - Funções que definem a sequência $\mathbf{N}_{\mathbf{D k}}$.

$\omega$ - Rotação do sólido (de corpo rígido).

$\mathbf{S}$ - Matriz que armazena as funções aproximadoras para a combinação linear de $\boldsymbol{\sigma}$ no domínio do elemento híbrido-Trefftz.

$\mathbf{S}_{\mathbf{i}}$ - Vetor de funções aproximadoras que compõe $\mathbf{S}$.

$\mathrm{T}$ - Matriz que armazena as funções aproximadoras para a combinação linear de $\mathbf{t}$.

$\mathbf{T}_{\mathbf{i}}$ - Vetor de funções aproximadoras que compõe $\mathbf{T}$.

$\tilde{\mathbf{u}}$ - Vetor da aproximação dos deslocamentos no contorno estático do elemento híbridoTrefftz.

$\widetilde{\mathbf{U}}$ - Matriz que contém as funções de forma para a combinação linear de $\tilde{\mathbf{u}}$.

d - Vetor que contém os graus de liberdade para a combinação linear de $\tilde{\mathbf{u}}$.

$\mathrm{u}_{\mathrm{x} 1}, \mathrm{u}_{\mathrm{x} 2}, \mathrm{u}_{\mathrm{y} 1}, \mathrm{u}_{\mathrm{y} 2}-$ Graus de liberdade.

$\xi, \eta$ - Coordenadas adimensionais.

$\mathrm{n}_{\mathrm{c}}$ - Dimensão do vetor $\mathbf{c}$.

$\mathrm{n}_{\mathrm{d}}$ - Dimensão do vetor $\mathbf{d}$.

$\mathbf{K}_{\text {Hт }}$ - Matriz resultante da operação entre as matrizes $\mathbf{F}$ e $\mathbf{G}$.

$\tilde{\mathbf{u}}^{\mathrm{i}}, \tilde{\mathbf{u}}^{\mathrm{j}}$ - Vetor da aproximação dos deslocamentos no contorno estático dos elementos $i$ e $j$ respectivamente.

$\boldsymbol{q}_{\boldsymbol{\sigma} k l}$ - Vetor de forças nodais equivalentes provenientes de tensão.

$\boldsymbol{q}_{\boldsymbol{i} k l}$ - Vetor de forças nodais equivalentes provenientes do carregamento.

$\mathbf{d}_{\mathbf{t}}$ - Vetor de graus de liberdade em deslocamento do sistema global.

$\mathbf{q}_{\mathbf{t}}$ - Vetor de forças nodais equivalentes do sistema global.

$\beta_{0}$ - Base de aproximação inicial.

$\beta_{\mathrm{g}}$ - Base enriquecida genérica.

$\beta_{\mathrm{E}}$ - Base enriquecedora genérica.

$h_{j}$ - Funções enriquecedoras. 
$\beta_{1}$ - Base enriquecida com refinamento polinomial hierárquico.

$\beta_{2}$ - Base enriquecida com refinamento polinomial não hierárquico.

$\beta_{3}$ - Base enriquecida com funções trigonométricas.

$\tilde{\mathbf{u}}_{\mathbf{E}}$ - Vetor da aproximação dos deslocamentos em um contorno enriquecido.

$\widetilde{\mathbf{U}}_{\mathbf{E}}$ - Matriz que contém as funções de forma para a combinação linear de $\tilde{\mathbf{u}}_{\mathbf{E}}$.

$\mathbf{d}_{\mathbf{E}}$ - Vetor que contém os graus de liberdade para a combinação linear de $\tilde{\mathbf{u}}_{\mathbf{E}}$.

$\Delta \mathrm{u}_{\mathrm{xi}}, \Delta \mathrm{u}_{\mathrm{yi}}$ - Graus de liberdade decorrentes do enriquecimento.

$\widetilde{\mathbf{U}}_{\Gamma_{\mathrm{E}}}$ - Matriz que contém as funções de forma $\phi_{1}$ e $\phi_{2}$.

$\Delta \widetilde{\mathbf{U}}_{\Gamma_{\mathrm{E}}}$ - Matriz que contém as bases de aproximação provenientes do enriquecimento.

$\mathbf{d}_{\Gamma_{\mathrm{E}}}$ - Vetor que contém os graus de liberdade iniciais.

$\Delta \mathbf{d}_{\Gamma_{\mathrm{E}}}$ - Vetor que contém os novos parâmetros decorrentes do enriquecimento.'

$\mathbf{G}_{\mathbf{E}}$ - Matriz $\mathbf{G}$ do elemento enriquecido.

$\mathbf{G}_{\Gamma_{\mathbf{E}}}$ - Parcela de $\mathbf{G}_{\mathbf{E}}$ referente à aproximação inicial.

$\Delta \mathbf{G}_{\Gamma_{\mathbf{E}}}$ - Parcela de $\mathbf{G}_{\mathbf{E}}$ decorrente do enriquecimento.

$\mathbf{q}_{\mathbf{E}}$ - Vetor $\mathbf{q}$ do elemento enriquecido.

$\mathbf{q}_{\Gamma_{\mathbf{E}}}$ - Parcela de $\mathbf{q}_{\mathbf{E}}$ referente à aproximação inicial.

$\Delta \mathbf{q}_{\Gamma_{\mathrm{E}}}$ - Parcela de $\mathbf{q}_{\mathbf{E}}$ decorrente do enriquecimento.

$\Delta \mathbf{d}$ - Vetor que coleta os graus de liberdade decorrentes do enriquecimento do elemento. $\Delta \mathbf{q}$ - Vetor que coleta os graus de liberdade decorrentes do enriquecimento do elemento.

$\Delta \mathbf{d}_{\mathrm{t}}$ - Vetor que coleta os graus de liberdade decorrentes do enriquecimento do sistema global.

$\Delta \mathbf{q}_{\mathbf{t}}$ - Vetor que coleta os graus de liberdade decorrentes do enriquecimento do sistema global.

$\phi_{j}(x)$ - Função PU no nó j.

$\mathbb{F}_{n}$ - Família de funções de forma do MEFG.

$\Phi$ - Função resultante do produto entre a PU e a função enriquecedora.

$\mathrm{x}_{\mathrm{i}}, \mathrm{y}_{\mathrm{i}}$ - Coordenadas do no $i$ do elemento.

J - Matriz Jacobiana. 
$\mathrm{H}$ - Matriz inversa da matriz Jacobiana.

B $^{*}$ - Matriz que coleta as derivadas parciais em relação a $\xi$ e $\eta$ das funções de forma.

B - Matriz que relaciona as deformações aos graus de liberdade.

$\mathbf{K}_{\mathbf{e}}$ - Matriz de Rigidez do elemento.

$\mathbf{r}$ - Vetor de cargas nodais.

$f_{j}^{u}$ - Função enriquecedora no nó $j$ na direção $u$.

$f_{j}^{v}$ - Função enriquecedora no nó $j$ na direção $v$.

$b_{j}^{u}$ - Função enriquecedora no nó $j$ na direção $u$.

$b_{j}^{v}$ - Função enriquecedora no nó $j$ na direção $v$.

$\mathbf{B}_{\mathbf{e}}^{*}$ - Matriz $\mathbf{B}^{*}$ enriquecida.

$P_{G}^{(0)}$ - Subproblema global.

$\boldsymbol{t}_{\boldsymbol{G}}^{(\mathbf{0})}$ - Tensões nas linhas que representam as fissuras do subproblema global.

$b_{i, j}$ - Constantes da combinação linear de $\boldsymbol{t}_{\boldsymbol{G}}^{(\mathbf{0})}$.

$Q_{j}\left(\xi_{i} / a_{i}\right)$ - Termos de aproximação.

$a_{i}$ - Comprimento da fissura $i$.

$P_{L}^{(k)}$ - Subproblemas locais.

$t_{L}^{(k)}$ - Tensões nas linhas que representam as fissuras dos subproblemas locais.

$P_{G}^{(k)}$ - Subproblemas globais.

$\boldsymbol{t}_{G}^{(k)}$ - Tensões nas linhas que representam as fissuras dos subproblemas globais.

$c_{i, j}^{(k)}$ - Constantes para a combinação linear.

$P_{G}$ - Problema global.

$\alpha$ - Fator de escala para a determinação do Fator de Intensidade de Tensão.

$M$ - Número máximo de problemas $P_{L}^{(k)}$ e $P_{G}^{(k)}$.

$Q_{j 2}\left(\xi_{i} / a_{i}\right)$ - Função ponderadora.

$[I G]$ - Matriz de influência geral.

$K^{(i)}$ - Fator de intensidade final.

$K_{j}^{(i)}$ - Fator de intensidade de tensão calculado no $P_{L}^{(k)}$. 
$G$ - Módulo de elasticidade transversal.

$F_{x}, F_{y}$ - Funções formadas pela solução analítica da mecânica da fratura na direção x e y, respectivamente.

$\kappa$ - Constante utilizada na determinação do Fator de Intensidade de Tensão.

$\Delta \varsigma$ - Deslocamento entre duas faces da fissura. 


\section{Sumário}

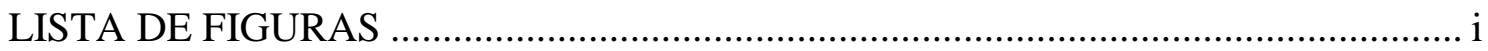

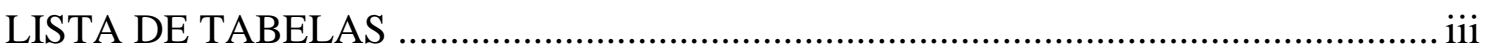

LISTA DE ABREVIATURAS E SIGLAS ….................................................... iv

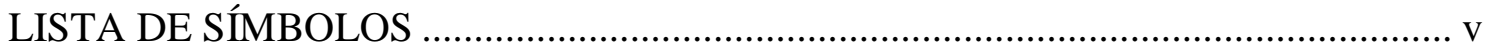

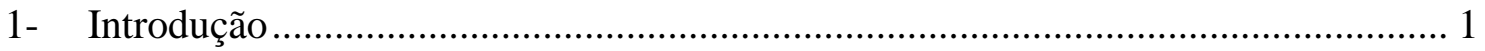

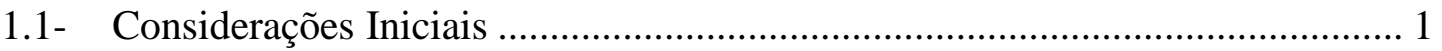

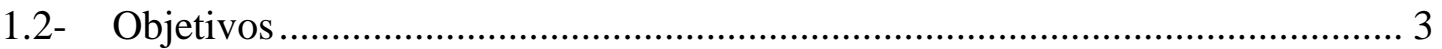

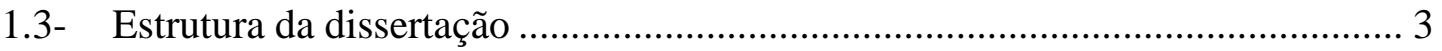

2- $\quad$ Formulações não-convencionais de elementos finitos .......................................... 5

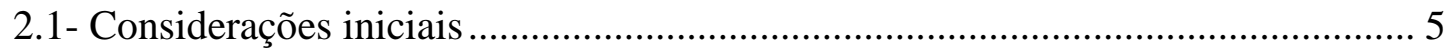

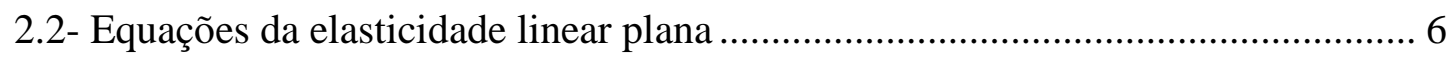

2.3- Equações da elasticidade linear plana em resíduos ponderados ........................... 8

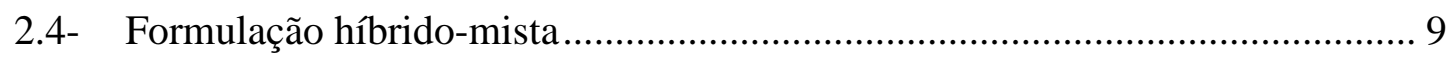

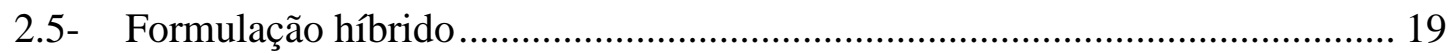

3- Formulação híbrido-Trefftz de tensão ............................................................. 21

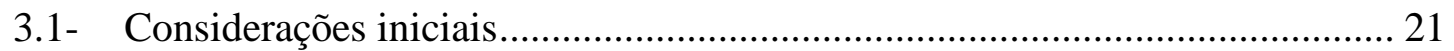

3.2- Elemento finito híbrido-Trefftz de tensão..................................................... 22

3.2.1- Equação de Navier ................................................................................... 23

3.2.2- Aproximação do campo de deslocamentos no domínio do elemento atendendo a equação de Navier .......................................................................... 24

3.2.3- Aproximação do campo de tensões no domínio ......................................... 28

3.2.4- Aproximação do campo de tensões no contorno.......................................... 29

3.2.5- Aproximação do campo de deslocamentos no contorno ............................. 29

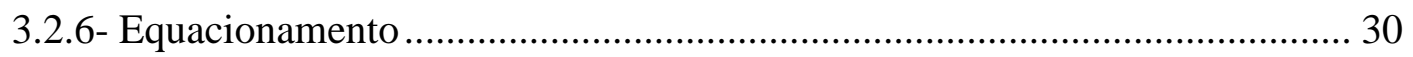

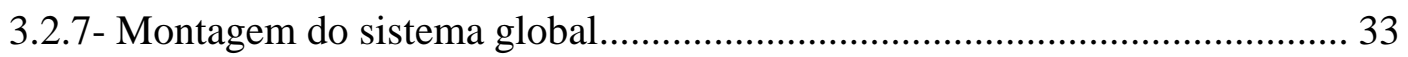


3.3- Elemento híbrido-Trefftz de tensão com enriquecimento seletivo

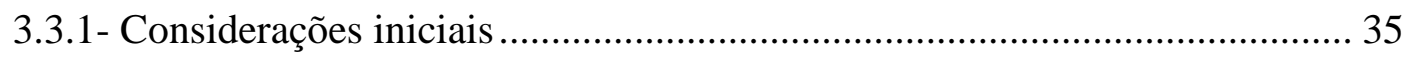

3.3.2- Bases enriquecedoras ...................................................................... 36

3.3.3- Aproximação do campo de deslocamento numa fronteira enriquecida ....... 40

3.3.4- Equacionamento levando em conta o enriquecimento ............................ 41

3.3.5- Montagem do sistema global considerando o enriquecimento ................... 43

4- $\quad$ O Método dos Elementos Finitos Generalizados ............................................... 47

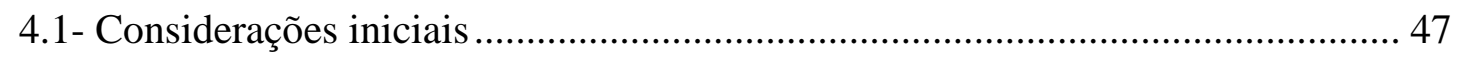

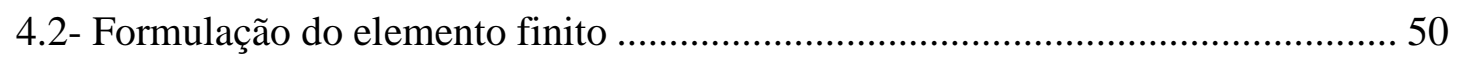

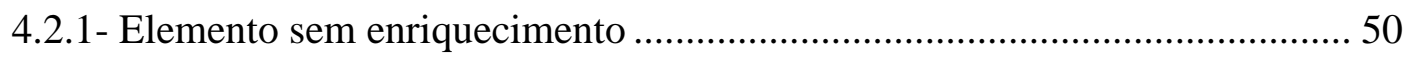

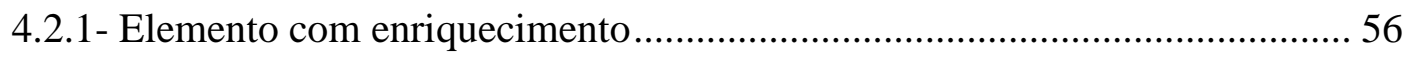

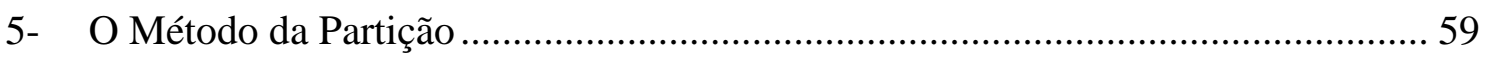

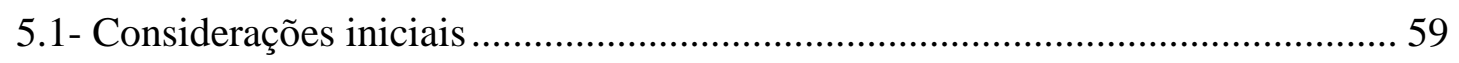

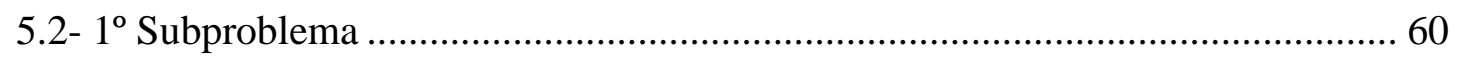

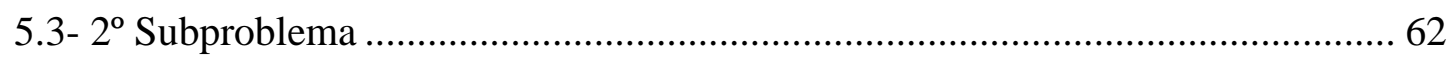

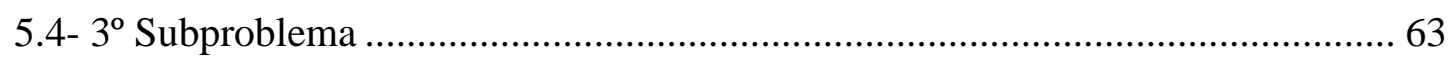

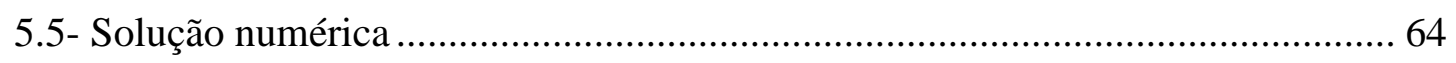

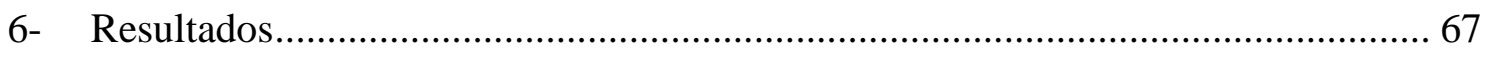

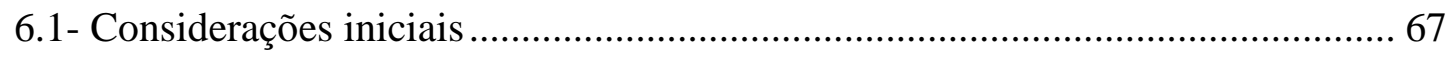

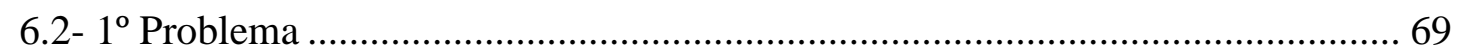

6.2.1 - Análise utilizando a formulação híbrido-Trefftz .................................... 70

6.2.2 - Análise utilizando o MEFG .............................................................. 74

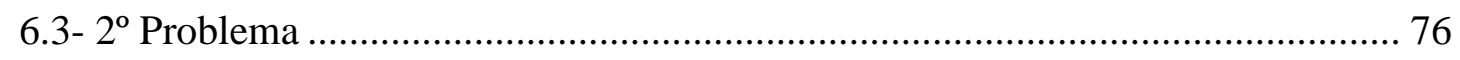

6.3.1 - Análise utilizando a formulação híbrido-Trefftz .................................... 77

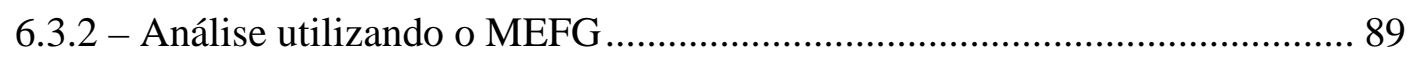

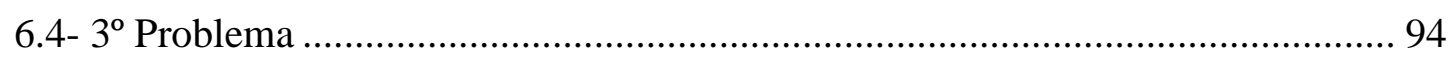

6.4.1- Análise utilizando a formulação híbrido-Trefftz....................................... 95 
6.4.2 - Análise utilizando o MEFG....

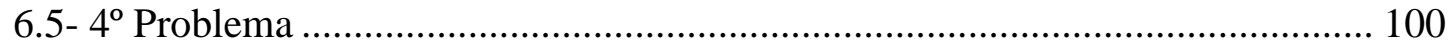

6.5.1 - Análise utilizando a formulação híbrido-Trefftz..................................... 102

6.5.2 - Análise utilizando o MEFG ............................................................... 106

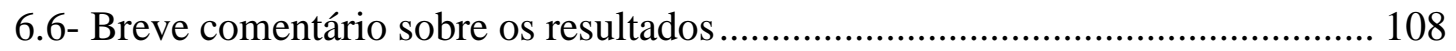

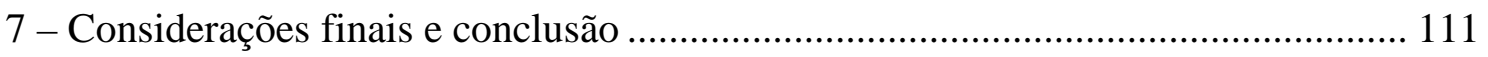

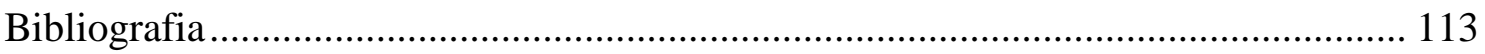

Anexo A - Matrizes utilizadas na aproximação dos campos de deslocamentos e tensões em domínios contínuos: Construção a partir da Equação de Navier.

Anexo B - Dedução do sistema linear final do Método da Partição. 



\section{1- Introdução}

\section{1- Considerações Iniciais}

Um dos grandes desafios do engenheiro estrutural é a concepção de um modelo matemático capaz de prever com relativa precisão seu comportamento real da estrutura. Em geral, tal modelo é regido por equações matemáticas que representam três condições básicas: de equilíbrio, de compatibilidade e constitutiva. Essas equações têm por incógnitas componentes de três campos importantes para a análise da estrutura: os campos de deslocamento, de tensão e de deformação.

As equações matemáticas devem ainda atender a certas restrições dadas pelas condições de contorno da estrutura. Por esse motivo, problemas desse tipo são denominados problema de valor de contorno (PVC). Apesar das hipóteses simplificadoras, a solução analítica do PVC é restrita a poucos problemas estruturais. Diante desse entrave a busca de soluções aproximativas justifica-se plenamente.

Os métodos numéricos fornecem a solução aproximada do problema em pontos da estrutura que constituem sua discretização. Dentre estes métodos existentes na literatura, o Método dos Elementos Finitos (MEF) com formulação em deslocamento é o mais utilizado dada sua simplicidade e capacidade de proporcionar soluções próximas da solução analítica.

Apesar disso, o MEF pode apresentar alguns percalços:

- Sensibilidade à distorção dos elementos;

- O emprego de funções polinomiais para a construção da aproximação da solução exige um grande refinamento da rede em problemas que contenham singularidades, elevando o custo computacional;

- Perda de precisão nos níveis superiores de derivadas da aproximação como, por exemplo, ao determinar os campos de deformações e de tensões devidos à derivação da solução em deslocamento. 
Entre as alternativas para superar tais limitações destacam-se as formulações nãoconvencionais de elementos finitos: formulação híbrido-mista, híbrido e híbrido-Trefftz (FREITAS; ALMEIDA; PEREIRA, 1999). Tais formulações podem ser obtidas a partir da ponderação independente das equações de equilíbrio, de compatibilidade e constitutiva do modelo mecânico. Observa-se que tais formulações são ditas híbridos porque aproximam campos independentes de deslocamento, tensão (ou deformação) no domínio e deslocamento ou tensão no contorno do elemento.

Por outro lado, os Métodos Sem Malhas (MSM) fornecem uma alternativa para a construção de aproximações da solução que dispensa o uso de elementos finitos. Já o Método dos Elementos Finitos Generalizados (MEFG) (DUARTE; BABUŠKA; ODEN, 2000) essencialmente combina as alternativas na medida em que consiste na união do MEF com a idéia de enriquecimento seletivo nodal proposto pelos Métodos Sem Malhas. O enriquecimento nodal com funções especiais permite captar elevados gradientes da solução, típicas dos problemas de fratura.

Por apresentar um elevado gradiente de tensões na ponta da fissura, esses problemas exigem um grande refinamento da rede ao utilizar o MEF. Já com as formulações nãoconvencionais como o MEFG, pode-se abrir mão desse grande refinamento, pois a aproximação nodal enriquecida é mais eficiente para captar o elevado gradiente de tensões.

Mais recentemente as formulações numéricas não-convencionais tem sido estendidas para tratar problemas com múltiplas fissuras (SOUZA; PROENÇA, 2009; DUARTE; KIM; BABUŠKA, 2007)

Um dos métodos para a análise dessa classe de problemas é o Método da Partição ("splitting method") (ANDERSSON; BABUŠKA; STEHLIN, 1998; BABUŠKA; ANDERSSON, 2005). Este método propõe a busca de solução dividindo o problema em três subproblemas: subproblema global $\left(P_{G}^{(0)}\right)$, subproblemas locais $\left(P_{L}^{(k)}\right)$ e subproblemas globais $\left(P_{G}^{(k)}\right)$. Sobrepondo a solução de cada um dos subproblemas tem-se a solução do problema global $P_{G}$.

O Método da Partição, a formulação híbrido-Trefftz e o MEFG, utilizados no presente trabalho, serão detalhados no decorrer do texto. 


\section{2- Objetivos}

Esse trabalho aborda fundamentalmente os problemas de sólidos contendo múltiplas fissuras segundo uma metodologia descrita pelo Método da Partição (MP) e com o emprego da formulação híbridra-Trefftz de tensão, com alternativa de enriquecimento proposta por SOUZA (2008).

Além disso, de modo original, insere-se o MEFG no Método da Partição a fim de ampliar as possibilidades de busca de soluções numéricas por elementos finitos. Para a análise utilizando o MEFG, buscou-se explorar o uso de funções analíticas de problemas da mecânica da fratura como funções enriquecedoras.

Nesse estudo não se trata da questão de propagação das fissuras, porém, tendo-se em vista sua consideração futura, uma grandeza de interesse a determinar é o fator de intensidade de tensão (FIT), particulamente atrelado ao modo I de abertura das fissuras. Os valores obtidos em cada análise são comparados com valores analíticos ou com valores obtidos pelo MEF convencional.

Para avaliar o desempenho de cada alternativa numérica, faz-se uma comparação entre o número de graus de liberdade usados na análise dos problemas resolvidos pela formulação híbrido-Trefftz, pelo MEFG e pelo MEF.

\section{3- Estrutura da dissertação}

Incluindo-se este capítulo de introdução, a estrutura da dissertação é composta de sete capítulos, tendo como intuito conceituar cada formulação adotada nesse trabalho, detalhar cada método empregado e expor os resultados finais. Os capítulos que seguem são compostos da seguinte forma:

- O capítulo 2 esclarece os conceitos básicos sobre as formulações nãoconvencionais dos PVC, mais especificamente a formulação híbrido-mista e 
formulação híbrido. As equações relativas à Teoria da Elasticidade são expostas, bem como o equacionamento de cada formulação citada.

- O capítulo 3 fornece detalhes sobre a formulação híbrido-Trefftz de tensão. Inicialmente é apresentada uma breve revisão bibliográfica sobre o tema e em seguida são expostas suas vantagens. Seu equacionamento é descrito tanto para o caso de elemento sem enriquecimento quanto para o caso de elemento enriquecido (SOUZA, 2008).

- O capítulo 4 é destinado aos comentários sobre o MEFG. Uma breve revisão bibliográfica é feita, abordando conceitos comuns aos Métodos Sem Malhas. Em seguida sua formulação é apresentada tomando-se por base um elemento finito quadrilateral isoparamétrico de quatro nós.

- O capítulo 5 apresenta o Método da Partição, sendo esse método ilustrado por um dos problemas resolvidos neste trabalho.

- O capítulo 6 expõe os exemplos numéricos analisados no trabalho.

- O capítulo 7 é referente às considerações finais e conclusões. 


\section{2- Formulações não-convencionais de elementos finitos}

\section{1- Considerações iniciais}

O presente capítulo faz uma breve discussão sobre formulações não-convencionais de elementos finitos, focando suas características básicas e suas vantagens em relação aos elementos finitos convencionais. A partir das equações do modelo elástico linear são apresentadas as formulações para duas classes de elementos finitos não-convencionais: os elementos híbrido-misto e híbrido. Tal abordagem é baseada no trabalho de Proença (2008). O elemento híbrido-Trefftz, foco principal da pesquisa, será analisado com mais detalhes no capítulo seguinte.

Por não-convencionais denominam-se as formulações em elementos finitos que não se baseiam em princípios variacionais e sim no método dos resíduos ponderados. São três as que se enquadram nessa categoria: as formulações híbrido-mista, híbrido e híbrido-Trefftz (FREITAS; ALMEIDA; PEREIRA, 1999). Os elementos delas derivados se caracterizam por aproximar de forma independente os campos de tensão e de deslocamento (ou deformação) no domínio e no contorno do elemento.

Tais formulações são utilizadas a fim de corrigir alguns entraves apresentados pelo MEF convencional como, por exemplo, a sensibilidade à distorção de forma. Além disso, em problemas que contenham fissuras (caracterizado por elevados gradientes de tensão em suas pontas), os elementos híbridos-Trefftz viabilizam a obtenção de resultados precisos sem a necessidade de um refinamento excessivo da rede.

A diferença básica entre as três formulações está na restrição quanto ao atendimento de condições de equilíbrio e compatibilidade imposta em forma forte aos campos de aproximação no domínio do elemento. Na formulação híbrido-mista todas as condições são atendidas em forma fraca. A híbrido é um caso particular da híbrido-mista, sendo obtida pela restrição da aproximação do domínio a fim de satisfazer localmente a equação de equilíbrio ou de compatibilidade. Já a formulação híbrido-Trefftz é obtida através da formulação híbrido 
impondo à aproximação do domínio o atendimento à equação diferencial governativa do problema (equação de Navier).

Cada formulação pode ser ainda desenvolvida utilizando dois modelos: de tensão e de deslocamento, de acordo com a forma como se compatibilizam os elementos ao longo de suas fronteiras. Ao utilizar o modelo de tensão, o equilíbrio de forças entre elementos é imposto em forma fraca e a compatibilidade de deslocamentos entre elementos é imposta em forma forte. Ao contrário, o modelo de deslocamento impõe em forma forte o equilíbrio de forças entre elementos e em forma fraca a compatibilidade de deslocamento.

\section{2- Equações da elasticidade linear plana}

Antes de apresentar a formulação dos elementos não-convencionais são revistas as equações que governam o PVC em forma forte da elasticidade linear plana (TIMOSHENKO; GOODIE, 1980).

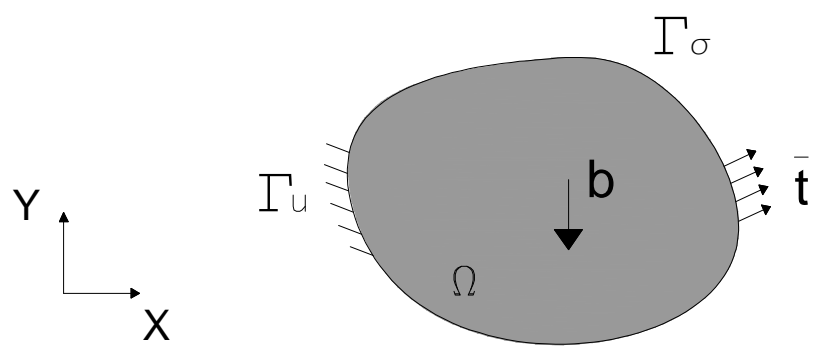

Figura 2.1 - Representação genérica de um sólido bidimensional.

A Figura 2.1 representa genericamente um sólido bidimensional. $\mathrm{O}$ vetor $\boldsymbol{b}$ guarda as componentes das forças de volume. O símbolo $\Omega$ representa o volume e $\Gamma$ o contorno do sólido, sendo $\Gamma_{\sigma}$ o contorno estático, onde forças prescritas $\bar{t}$ são impostos e $\Gamma_{u}$ o contorno cinemático, onde deslocamentos $\bar{u}$ são prescritos. As regiões de contorno são complementares, de modo que a fronteira $\Gamma$ pode ser representada pela seguinte expressão: $\Gamma=\Gamma_{\sigma} \cup \Gamma_{u}$.

As equações básicas do PVC são: 


$$
\begin{array}{cc}
\boldsymbol{L} \boldsymbol{\sigma}+\overline{\boldsymbol{b}}=\mathbf{0} & \text { em } \Omega \\
\boldsymbol{\varepsilon}=\boldsymbol{L}^{\mathrm{T}} \boldsymbol{u} & \text { em } \Omega \\
\boldsymbol{\varepsilon}=\boldsymbol{f} \boldsymbol{\sigma} & \text { em } \Omega \\
\text { ou } \boldsymbol{\sigma}=\boldsymbol{D} \boldsymbol{\varepsilon} & \text { em } \Omega \\
\boldsymbol{I} \boldsymbol{\sigma}=\overline{\boldsymbol{t}} & \text { em } \Gamma_{\sigma} \\
\boldsymbol{u}=\overline{\boldsymbol{u}} & \text { em } \Gamma_{u}
\end{array}
$$

A Equação (2.1) é a equação que exprime o equilíbrio no domínio. A matriz $\boldsymbol{L}$ reúne operadores diferenciais, o vetor $\boldsymbol{\sigma}$ contém as componentes do tensor de tensões e $\overline{\boldsymbol{b}}$ o vetor das forças volúmicas. Tais elementos são representados por:

- $\quad \boldsymbol{L}=\left[\begin{array}{ccc}\partial / \partial x & 0 & \partial / \partial y \\ 0 & \partial / \partial y & \partial / \partial x\end{array}\right]$, sendo $\partial / \partial x$ e $\partial / \partial y$ derivadas parciais;

- $\boldsymbol{\sigma}^{\mathrm{T}}=\left\{\begin{array}{lll}\sigma_{x} & \sigma_{y} & \tau_{x y}\end{array}\right\}$, sendo $\sigma_{x}$ e $\sigma_{y}$ as componentes de tensão normal e $\tau_{x y}$ a componente cisalhante;

- $\boldsymbol{b}^{\mathrm{T}}=\left\{\begin{array}{ll}b_{x} & b_{y}\end{array}\right\}$, sendo $b_{x}$ e $b_{y}$ as componentes do vetor segundo os eixos de referência.

A Equação (2.2) é dita equação de compatibilidade, onde $\boldsymbol{\varepsilon}$ é o vetor representativo do tensor de deformações e $\boldsymbol{u}$ é o vetor de deslocamentos. Esses elementos são representados por:

- $\boldsymbol{\varepsilon}^{\mathrm{T}}=\left\{\begin{array}{lll}\varepsilon_{x} & \varepsilon_{y} & \gamma_{x y}\end{array}\right\}$, sendo $\varepsilon_{x}, \varepsilon_{y}$ componentes de deformação linear específica e $\gamma_{x y}$ a distorção angular;

- $\boldsymbol{u}^{\mathrm{T}}=\left\{\begin{array}{ll}u_{x} & u_{y}\end{array}\right\}$, sendo $u_{x}$ e $u_{y}$ componentes do vetor de deslocamento.

As equações (2.3) e (2.4) constituem alternativas para a relação constitutiva do sólido. A primeira é escrita em termos de $f$ que é o tensor constitutivo de flexibilidade, representado por: 
- $f=\frac{1}{\mathrm{E}}\left[\begin{array}{ccc}1 & -v & 0 \\ -v & 1 & 0 \\ 0 & 0 & 2(1+v)\end{array}\right]$, sendo E o módulo de elasticidade e $v$ o coeficiente de Poisson.

A segunda é escrita em termos de $\boldsymbol{D}$ que é o tensor constitutivo de rigidez, representado por:

$$
\text { - } \boldsymbol{D}=\frac{E}{1-v^{2}}\left[\begin{array}{ccc}
1 & v & 0 \\
v & 1 & 0 \\
0 & 0 & \frac{(1-v)}{2}
\end{array}\right]
$$

A relação (2.5) é denominada de condição de contorno de Neumann. A matriz I é formada pelas componentes de um vetor unitário normal à $\Gamma_{\sigma}$ e $\bar{t}$ é o vetor de forças de superfície prescritas em $\Gamma_{\sigma}$ (tensões de Cauchy). Essas grandezas são representadas por:

- $\boldsymbol{I}=\left[\begin{array}{ccc}\mathrm{n}_{\mathrm{x}} & 0 & \mathrm{n}_{\mathrm{y}} \\ 0 & \mathrm{n}_{\mathrm{y}} & \mathrm{n}_{\mathrm{x}}\end{array}\right]$, sendo $n_{x}$ e $n_{y}$ as componentes de um vetor unitário normal a $\Gamma_{\sigma}$;

- $\overline{\boldsymbol{t}}^{T}=\left\{\begin{array}{ll}\overline{\mathrm{t}}_{\mathrm{x}} & \overline{\mathrm{t}}_{\mathrm{y}}\end{array}\right\}$, sendo $\overline{\mathrm{t}}_{\mathrm{x}}$ e $\overline{\mathrm{t}}_{\mathrm{y}}$ as componentes do vetor de forças de superfície prescrita.

A Equação (2.6) é dita condição de contorno de Dirichlet. O vetor $\bar{u}$ é o vetor de deslocamentos prescritos no contorno $\Gamma_{u}$, representado por:

- $\overline{\boldsymbol{u}}=\left\{\begin{array}{ll}\bar{u}_{x} & \bar{u}_{y}\end{array}\right\}$, sendo $\bar{u}_{x}$ e $\bar{u}_{y}$ componentes do vetor de deslocamentos prescritos no contorno $\Gamma_{u}$;

\section{3- Equações da elasticidade linear plana em resíduos ponderados}

Com as relações da elasticidade linear, podem-se deduzir as expressões para as formulações não-convencionais. Inicialmente, utiliza-se o método dos resíduos ponderados nas equações (2.1), (2.2), (2.3), (2.5), admitindo-se que as restrições aos deslocamentos no contorno $\Gamma_{u}$ (Equação (2.6)) sejam previamente atendidas em forma forte. 
As expressões em resíduos ponderados são:

$$
\begin{gathered}
\int_{\Omega} \boldsymbol{M}_{\Omega}^{\mathrm{T}}\left(\boldsymbol{L}^{\mathrm{T}} \boldsymbol{u}-\boldsymbol{f} \boldsymbol{\sigma}\right) \mathrm{d} \Omega=0 \\
\int_{\Omega} \boldsymbol{P}_{\Omega}^{\mathrm{T}}(\boldsymbol{L} \boldsymbol{\sigma}+\overline{\boldsymbol{b}}) \mathrm{d} \Omega=0 \\
\int_{\Gamma_{\sigma}} \boldsymbol{V}_{\Gamma_{\sigma}}^{\mathrm{T}}(\overline{\boldsymbol{t}}-\boldsymbol{I} \boldsymbol{\sigma}) \mathrm{d} \Gamma=0
\end{gathered}
$$

Nota-se que a Equação (2.7) combina as relações de compatibilidade (2.2) e constitutiva do material (2.3). As funções peso são obtidas através das seguintes aproximações:

$$
\begin{gathered}
\boldsymbol{m}=\boldsymbol{M}_{\Omega}^{T} \boldsymbol{\delta} \boldsymbol{m}_{\Omega} \\
\boldsymbol{p}=\boldsymbol{P}_{\Omega}^{T} \boldsymbol{\delta} \boldsymbol{p}_{\Omega} \\
\boldsymbol{v}=\boldsymbol{V}_{\Gamma_{\sigma}}^{T} \boldsymbol{\delta} \boldsymbol{v}_{\Gamma_{\sigma}}
\end{gathered}
$$

Integrando por partes a Equação (2.7), obtém-se:

$$
\int_{\Omega}\left(\boldsymbol{L} \boldsymbol{M}_{\Omega}\right)^{\mathrm{T}} \boldsymbol{u} \mathrm{d} \Omega+\int_{\Omega} \boldsymbol{M}_{\Omega}^{\mathrm{T}} \boldsymbol{f} \boldsymbol{\sigma} \mathrm{d} \Omega-\int_{\Gamma_{\sigma}}\left(\boldsymbol{I}_{\Omega}\right)^{\mathrm{T}} \boldsymbol{u}_{\Gamma} \mathrm{d} \Gamma-\int_{\Gamma_{\mathrm{u}}}\left(\boldsymbol{I} \boldsymbol{M}_{\Omega}\right)^{\mathrm{T}} \overline{\boldsymbol{u}} \mathrm{d} \Gamma=0
$$

\section{4- Formulação híbrido-mista}

$\mathrm{Na}$ formulação híbrido-mista as aproximações dos campos de tensão e deslocamento são independentes no domínio (por isso é dita mista). Além disso, outra aproximação de deslocamentos é realizada no contorno $\Gamma_{\sigma}$ independente daquela do domínio (por isso é dita híbrido). Tais aproximações são representadas por:

$$
\begin{array}{cc}
\boldsymbol{\sigma}=\boldsymbol{S}_{\Omega} \boldsymbol{X} & \text { em } \Omega \\
\boldsymbol{u}=\boldsymbol{U}_{\Omega} \boldsymbol{q}_{\Omega} & \text { em } \Omega \\
\boldsymbol{u}_{\Gamma}=\boldsymbol{U}_{\Gamma_{\sigma}} \boldsymbol{q}_{\Gamma_{\sigma}} & \text { em } \Gamma_{\sigma}
\end{array}
$$


onde os vetores $\boldsymbol{\sigma}, \boldsymbol{u}$ e $\boldsymbol{u}_{\Gamma}$ definem os campos de tensões e deslocamentos no domínio e o campo de deslocamento no contorno estático, respectivamente. As matrizes $S_{\Omega}, \boldsymbol{U}_{\Omega}$ e $\boldsymbol{U}_{\Gamma_{\boldsymbol{\sigma}}}$ coletam as funções de aproximação da combinação linear e os vetores $\boldsymbol{X}, \boldsymbol{q}_{\Omega}$ e $\boldsymbol{q}_{\Gamma_{\sigma}}$ são os parâmetros a serem determinados.

Substituindo as três equações anteriores nas relações (2.13), (2.8) e (2.9), obtém-se o seguinte sistema linear:

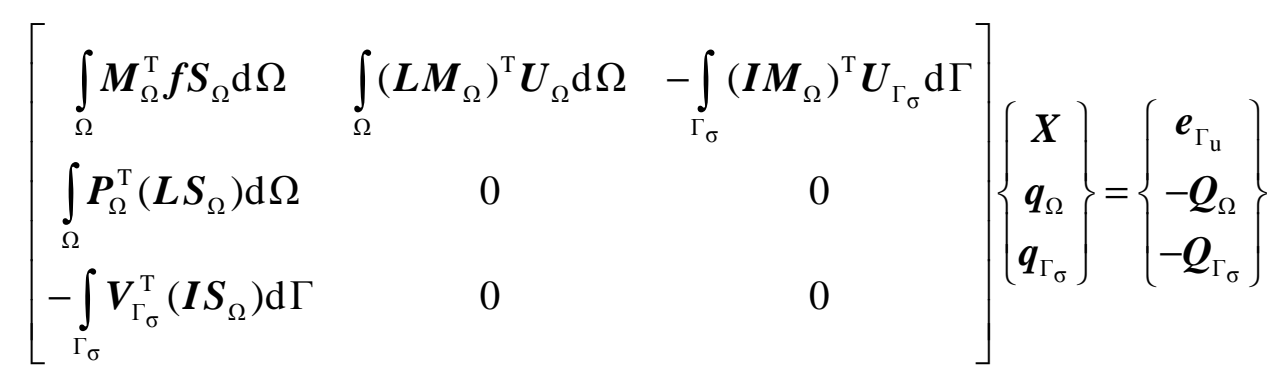

onde:

$$
\begin{gathered}
\boldsymbol{e}_{\Gamma_{\boldsymbol{u}}}=\int_{\Gamma_{\mathrm{u}}}\left(\boldsymbol{I}_{\Omega}\right)^{\mathrm{T}} \overline{\boldsymbol{u}} \mathrm{d} \Gamma \\
\boldsymbol{Q}_{\Omega}=\int_{\Omega} \boldsymbol{P}_{\Omega}^{\mathrm{T}} \overline{\boldsymbol{b}} \mathrm{d} \Omega \\
\boldsymbol{Q}_{\Gamma_{\sigma}}=\int_{\Gamma_{\sigma}} \boldsymbol{V}_{\Gamma_{\sigma}^{\mathrm{T}}}^{\mathrm{T}} \mathrm{d} \Gamma
\end{gathered}
$$

A fim de garantir a simetria das matrizes que compõem o sistema, adotam-se as seguintes aproximações de Galerkin:

$$
\begin{gathered}
\boldsymbol{M}_{\Omega}=\boldsymbol{S}_{\Omega} \\
\boldsymbol{P}_{\Omega}=\boldsymbol{U}_{\Omega} \\
\boldsymbol{V}_{\Gamma_{\sigma}}=\boldsymbol{U}_{\Gamma_{\sigma}}
\end{gathered}
$$

Assim, o sistema pode ser escrito da seguinte forma: 


$$
\left[\begin{array}{ccc}
\boldsymbol{F} & \boldsymbol{G}_{\Omega} & -\boldsymbol{G}_{\Gamma_{\sigma}} \\
\boldsymbol{G}_{\Omega}^{\mathrm{T}} & 0 & 0 \\
-\boldsymbol{G}_{\Gamma_{\sigma}}^{\mathrm{T}} & 0 & 0
\end{array}\right]\left\{\begin{array}{c}
\boldsymbol{X} \\
\boldsymbol{q}_{\Omega} \\
\boldsymbol{q}_{\Gamma_{\sigma}}
\end{array}\right\}=\left\{\begin{array}{c}
\boldsymbol{e}_{\Gamma_{\mathrm{u}}} \\
-\boldsymbol{Q}_{\Omega} \\
-\boldsymbol{Q}_{\Gamma_{\sigma}}
\end{array}\right\}
$$

Onde:

$$
\begin{gathered}
\boldsymbol{F}=\int_{\Omega} \boldsymbol{S}_{\Omega}^{\mathrm{T}} \boldsymbol{f} \boldsymbol{S}_{\Omega} \mathrm{d} \Omega \\
\boldsymbol{G}_{\Omega}=\int_{\Omega}\left(\boldsymbol{L} \boldsymbol{S}_{\Omega}\right)^{\boldsymbol{T}} \boldsymbol{U}_{\Omega} \mathrm{d} \Omega \\
\boldsymbol{G}_{\Gamma_{\sigma}}=\int_{\Gamma_{\sigma}}\left(\boldsymbol{I} \boldsymbol{S}_{\Omega}\right)^{\mathrm{T}} \boldsymbol{U}_{\Gamma_{\sigma}} \mathrm{d} \Gamma \\
\boldsymbol{e}_{\Gamma_{\mathrm{u}}}=\int_{\Gamma_{\mathrm{u}}}\left(\boldsymbol{I} \boldsymbol{S}_{\Omega}\right)^{\mathrm{T}} \overline{\boldsymbol{u}} \mathrm{d} \Gamma \\
\boldsymbol{Q}_{\Omega}=\int_{\Omega} \boldsymbol{U}_{\Omega}^{\mathrm{T}} \overline{\boldsymbol{b}} \mathrm{d} \Omega \\
\boldsymbol{Q}_{\Gamma_{\sigma}}=\int_{\Gamma_{\sigma}} \boldsymbol{U}_{\Gamma_{\sigma}^{\mathrm{T}}}^{\mathrm{T}} \overline{\boldsymbol{t}} \mathrm{d} \Gamma
\end{gathered}
$$

Quando do emprego do MEF, deve-se garantir que o modelo discretizado represente bem o modelo contínuo, ou seja, que condições de continuidade sejam obedecidas entre elementos. Mais especificamente, deve-se garantir que as condições de equilíbrio e compatibilidade sejam atendidas na fronteira comum entre os elementos.

Considerando-se um elemento isolado, os deslocamentos e forças na fronteira interior $\Gamma_{\mathrm{i}}$ (Figura 2.2) são desconhecidos, constituindo-se em novas incógnitas do sistema. Portanto, elas também podem ser aproximadas por campos independentes:

$$
\begin{gathered}
\boldsymbol{t}_{\Gamma_{\mathrm{u}}}=\boldsymbol{W}_{\Gamma_{\mathrm{u}}} \boldsymbol{z}_{\Gamma_{\mathrm{u}}} \text { em } \Gamma_{\mathrm{u}} \\
\boldsymbol{u}_{\Gamma_{\mathrm{i}}}=\boldsymbol{U}_{\Gamma_{\mathrm{i}}} \boldsymbol{q}_{\Gamma_{\mathrm{i}}} \text { em } \Gamma_{\mathrm{i}} \\
\boldsymbol{t}_{\Gamma_{\mathrm{i}}}=\boldsymbol{W}_{\Gamma_{\mathrm{i}}} \boldsymbol{z}_{\Gamma_{\mathrm{i}}} \text { em } \Gamma_{\mathrm{i}}
\end{gathered}
$$




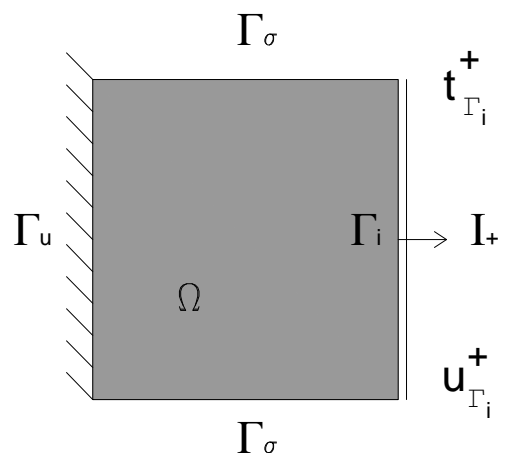

Figura 2.2 - Elemento finito contendo $\Gamma_{\mathrm{i}}$.

As condições de compatibilidade e equilíbrio (2.24) devem ser estendidas de modo a incluir o contorno $\Gamma_{\mathrm{i}}$. Nesse sentido, uma relação análoga a (2.9) é adicionada ao sistema e a Equação (2.13) passa a ter uma integral a mais. Tal desenvolvimento leva ao seguinte sistema:

$$
\left[\begin{array}{cccc}
\boldsymbol{F} & \boldsymbol{G}_{\Omega} & -\boldsymbol{G}_{\Gamma_{\sigma}} & -\boldsymbol{G}_{\Gamma_{\mathrm{i}}} \\
\boldsymbol{G}_{\Omega}^{\boldsymbol{T}} & 0 & 0 & 0 \\
-\boldsymbol{G}_{\Gamma_{\sigma}}^{\boldsymbol{T}} & 0 & 0 & 0 \\
-\boldsymbol{G}_{\Gamma_{\mathrm{i}}}^{\boldsymbol{T}} & 0 & 0 & 0
\end{array}\right]\left\{\begin{array}{c}
\boldsymbol{X} \\
\boldsymbol{q}_{\Omega} \\
\boldsymbol{q}_{\Gamma_{\sigma}} \\
\boldsymbol{q}_{\Gamma_{\mathrm{i}}}^{+}
\end{array}\right\}=\left\{\begin{array}{c}
\boldsymbol{e}_{\Gamma_{\mathrm{u}}} \\
-\boldsymbol{Q}_{\Omega} \\
-\boldsymbol{Q}_{\Gamma_{\sigma}} \\
-\boldsymbol{Q}_{\Gamma_{\mathrm{i}}}^{+}
\end{array}\right\}
$$

As novas matrizes do sistema valem:

$$
\begin{gathered}
\boldsymbol{Q}_{\Gamma_{\mathrm{i}}}^{+}=\int_{\Gamma_{\mathrm{i}}} \boldsymbol{U}_{\Gamma_{\mathrm{i}}}^{\mathrm{T}} \boldsymbol{t}_{\Gamma_{\mathrm{i}}}^{+} \mathrm{d} \Gamma \\
\boldsymbol{G}_{\Gamma_{\mathrm{i}}}=\int_{\Gamma_{\mathrm{i}}}\left(\boldsymbol{I}_{+} \boldsymbol{S}_{\Omega}\right)^{\mathrm{T}} \boldsymbol{U}_{\Gamma_{\mathrm{i}}} \mathrm{d} \Gamma
\end{gathered}
$$

Vale observar que $\boldsymbol{t}_{\Gamma_{\mathrm{i}}}^{+}$não é conhecida, portanto o sistema (2.34) envolve um conjunto de cinco vetores incógnitos e quatro equações.

Quando da montagem do sistema correspondente a uma rede de elementos, condições adicionais devem ser impostas em relação aos contornos internos $\Gamma_{\mathrm{i}}$ :

- Os deslocamentos devem atender a uma condição de continuidade;

- As tensões devem atender a uma condição de reciprocidade. 
Para diminuir o número de incógnitas do sistema, uma das condições acima pode ser imposta em forma forte e a outra em forma fraca. A depender do campo escolhido para se impor a forma fraca, o contorno $\Gamma_{\mathrm{i}}$ será nomeado de contorno de Neumann (ou contorno estático) ou de Dirichlet (ou contorno cinemático).

Antes de apresentar o equacionamento para dois elementos vizinhos, é interessante analisar o significado de cada equação do sistema (2.34).

A primeira equação representa o resultado da integração por partes das equações de compatibilidade e constitutiva com o acréscimo da parcela que representa a compatibilização $\operatorname{com} \Gamma_{\mathrm{i}}$ (Equação (2.37)).

$$
\int_{\Omega} \boldsymbol{S}_{\Omega}^{\mathrm{T}} \boldsymbol{f} \sigma \mathrm{d} \Omega=-\int_{\Omega}\left(\boldsymbol{L} \boldsymbol{S}_{\Omega}\right)^{\mathrm{T}} \boldsymbol{u} \mathrm{d} \Omega+\int_{\Gamma_{\sigma}}\left(\boldsymbol{I} \boldsymbol{S}_{\Omega}\right)^{\mathrm{T}} \boldsymbol{u}_{\Gamma_{\sigma}} \mathrm{d} \Gamma+\int_{\Gamma_{\mathrm{i}}}\left(\boldsymbol{I}_{+} \boldsymbol{S}_{\Omega}\right)^{\mathrm{T}} \boldsymbol{u}_{\Gamma_{\mathrm{i}}} \mathrm{d} \Gamma+\int_{\Gamma_{\mathrm{u}}}\left(\boldsymbol{I} \boldsymbol{S}_{\Omega}\right)^{\mathrm{T}} \overline{\boldsymbol{u}} \mathrm{d} \Gamma
$$

Introduzindo as aproximações (2.14), (2.15), (2.16) e (2.32), a equação acima, resulta em:

$$
\boldsymbol{F} \boldsymbol{X}+\boldsymbol{G}_{\Omega} \boldsymbol{q}_{\Omega}-\boldsymbol{G}_{\Gamma_{\sigma}} \boldsymbol{q}_{\Gamma_{\sigma}}-\boldsymbol{G}_{\Gamma_{i}} \boldsymbol{q}_{\Gamma_{i}}=\boldsymbol{e}_{\Gamma_{u}}
$$

Sendo as matrizes $\boldsymbol{F}, \boldsymbol{G}_{\Omega}, \boldsymbol{G}_{\Gamma_{\boldsymbol{\sigma}}}$ e $\boldsymbol{e}_{\Gamma_{\boldsymbol{u}}}$ definidas em (2.25), (2.26), (2.27) e (2.28), respectivamente, e $\boldsymbol{G}_{\Gamma_{i}}$ expressa por (2.36).

Normalmente, chama-se atenção que a condição de contorno em $\Gamma_{u}$ é atendida em forma forte.

Quanto à fronteira comum entre elementos, ela pode ser representada na seguinte forma:

$$
\int_{\Gamma_{\mathrm{i}}} U_{\Gamma_{\mathrm{i}}}^{T}\left(I_{+} \sigma-t_{\Gamma_{i}}^{+}\right) \mathrm{d} \Gamma=0
$$

Ao substituir a aproximação de tensões no domínio (Equação (2.14)) na equação acima, resulta a quarta equação do sistema:

$$
\boldsymbol{G}_{\Gamma_{i}}^{T} \boldsymbol{X}-\boldsymbol{Q}_{\Gamma_{i}}^{+}=0
$$

Sendo as matrizes $\boldsymbol{Q}_{\Gamma_{i}}^{+}$e $\boldsymbol{G}_{\Gamma_{i}}$ expressas em (2.35) e (2.36) respectivamente. 
As outras equações do sistema definido em (2.34) referem-se ao equilíbrio no volume e a condição de Neumann no contorno $\Gamma_{\sigma}$.

Agora, considere-se uma rede formada por dois elementos finitos ilustrados na Figura 2.3. A princípio, adotam-se as seguintes aproximações:

$$
\begin{gathered}
\boldsymbol{u}_{\Gamma_{i}}^{+}=\boldsymbol{U}_{\Gamma_{i}}^{+} \boldsymbol{q}_{\Gamma_{i}}^{+} \\
\boldsymbol{t}_{\Gamma_{i}}^{+}=\boldsymbol{W}_{\Gamma_{i}}^{+} \boldsymbol{o}_{\Gamma_{i}}^{+} \\
\boldsymbol{u}_{\Gamma_{i}}^{-}=\boldsymbol{U}_{\Gamma_{i}}^{-} \boldsymbol{q}_{\Gamma_{i}}^{-} \\
\boldsymbol{t}_{\Gamma_{i}}^{-}=\boldsymbol{W}_{\Gamma_{i}}^{-} \boldsymbol{o}_{\Gamma_{i}}^{-}
\end{gathered}
$$

Como tais aproximações são independentes, o sistema reunindo as equações de cada elemento apresenta um total de oito equações e dez incógnitas. As duas equações que precisam ser adicionadas ao sistema provém da condição de continuidade e reciprocidade em $\Gamma_{\mathrm{i}}$.

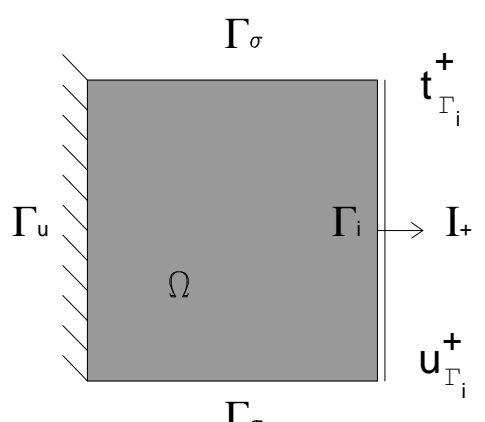

$\Gamma_{\sigma}$

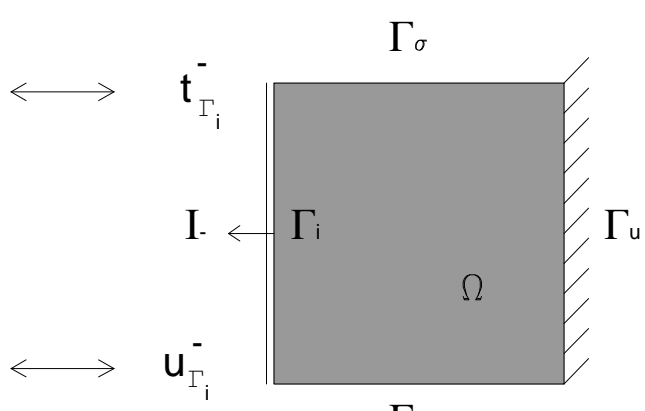

$\Gamma_{\sigma}$

Figura 2.3 - Elementos finitos vizinhos.

Pode-se recorrer a duas hipóteses para montar o sistema final do problema:

1. Impor a condição de reciprocidade em forma forte e a de continuidade em forma fraca. Assim, o contorno $\Gamma_{\mathrm{i}}$ é denominado de contorno de Dirichlet;

2. Impor a condição de continuidade em forma forte e a de reciprocidade em forma fraca, Assim, o contorno $\Gamma_{\mathrm{i}}$ é denominado de contorno de Neumann; 
Ao impor uma das condições em forma forte, o sistema passa a ter nove equações e nove incógnitas, tornando-se determinado.

A fim de equacionar o problema, considere-se a primeira hipótese, onde as forças em $\Gamma_{\mathrm{i}}$ passam a ser representadas por:

$$
\boldsymbol{t}_{\Gamma_{i}}^{+}=-\boldsymbol{t}_{\Gamma_{i}}^{-}=\boldsymbol{t}=\boldsymbol{W}_{\Gamma_{i}} \boldsymbol{o}_{\Gamma_{i}}
$$

As relações de equilíbrio nas fronteiras dos elementos são representadas por:

$$
\begin{aligned}
& \boldsymbol{G}_{\Gamma_{i}}^{+^{T}} \boldsymbol{X}^{1}=\boldsymbol{Q}_{\Gamma_{i}}^{+} \\
& \boldsymbol{G}_{\Gamma_{i}}^{-^{T}} \boldsymbol{X}^{2}=\boldsymbol{Q}_{\Gamma_{i}}^{-}
\end{aligned}
$$

sendo as matrizes $\boldsymbol{Q}_{\Gamma_{i}}^{+}$e $\boldsymbol{G}_{\Gamma_{i}}$ expressas em (2.35) e (2.36) respectivamente.

Devido à reciprocidade (Equação (2.45)), $\boldsymbol{Q}_{\Gamma_{\mathrm{i}}}^{+}$e $\boldsymbol{Q}_{\Gamma_{\mathrm{i}}}^{-}$passam a ser escritos da seguinte maneira:

$$
\begin{aligned}
& \boldsymbol{Q}_{\Gamma_{\mathrm{i}}}^{+}=\int_{\Gamma_{\mathrm{i}}^{+}} \boldsymbol{U}_{\Gamma_{\mathrm{i}}}^{+^{\mathrm{T}}} \boldsymbol{t} \mathrm{d} \Gamma \\
& \boldsymbol{Q}_{\Gamma_{\mathrm{i}}}^{-}=-\int_{\Gamma_{\mathrm{i}}^{-}} \boldsymbol{U}_{\Gamma_{\mathrm{i}}}^{-\mathrm{T}} \boldsymbol{t} \mathrm{d} \Gamma
\end{aligned}
$$

Substituindo (2.45) em (2.48) e (2.49), as formas lineares $\boldsymbol{Q}_{\Gamma_{\mathrm{i}}}^{+}$e $\boldsymbol{Q}_{\Gamma_{\mathrm{i}}}^{-}$para os elementos 1 e 2 são representadas por (2.50) e (2.51), respectivamente.

$$
\begin{gathered}
\boldsymbol{Q}_{\Gamma_{i}}^{+}=\underbrace{\left[\int_{\Gamma_{i}^{+}} \boldsymbol{U}_{\Gamma_{i}}^{+T} \boldsymbol{W}_{\Gamma_{i}} d \Gamma\right]}_{C_{\Gamma_{i}}^{T^{T}}} \boldsymbol{o}_{\Gamma_{i}}=\boldsymbol{C}_{\Gamma_{i}}^{+^{T}} \boldsymbol{o}_{\Gamma_{i}} \\
\boldsymbol{Q}_{\Gamma_{i}}^{-}=-\underbrace{\left[\int_{\Gamma_{i}^{-}} \boldsymbol{U}_{\Gamma_{i}}^{-T} \boldsymbol{W}_{\Gamma_{i}} d \Gamma\right]}_{c_{\Gamma_{i}}^{-T}} \boldsymbol{o}_{\Gamma_{i}}=-\boldsymbol{C}_{\Gamma_{i}}^{-T} \boldsymbol{o}_{\Gamma_{i}}
\end{gathered}
$$


Substituindo (2.50) em (2.46) e (2.51) em (2.47), obtêm-se as relações de equilíbrio para os elementos 1 e 2 , respectivamente:

$$
\begin{gathered}
\boldsymbol{G}_{\Gamma_{i}}^{+^{T}} \boldsymbol{X}^{1}-\boldsymbol{C}_{\Gamma_{i}}^{+^{T}} \boldsymbol{o}_{\Gamma_{i}}=0 \\
\boldsymbol{G}_{\Gamma_{\mathrm{i}}}^{-^{\mathrm{T}}} \boldsymbol{X}^{2}+\boldsymbol{C}_{\Gamma_{\mathrm{i}}}^{-^{\mathrm{T}}} \boldsymbol{o}_{\Gamma_{\mathrm{i}}}=0
\end{gathered}
$$

Tendo em mãos a equação final do equilíbrio devido à reciprocidade, deve-se impor a relação de continuidade em forma fraca. Para isso, utilizam-se forças virtuais como funções ponderadoras, expressa como:

$$
\int_{\Gamma_{\mathrm{i}}} \delta \boldsymbol{t}^{\mathrm{T}}\left(\boldsymbol{u}_{\Gamma_{\mathrm{i}}}^{+}-\boldsymbol{u}_{\Gamma_{\mathrm{i}}}^{-}\right) \mathrm{d} \Gamma=0
$$

Considerando a seguinte aproximação para as forças virtuais:

$$
\delta t=W_{\Gamma_{\mathrm{i}}} \delta p_{\Gamma_{\mathrm{i}}}
$$

Combinando as duas equações acima, têm-se:

$$
\delta \boldsymbol{p}_{\Gamma_{\mathrm{i}}}^{\mathrm{T}} \int_{\Gamma_{\mathrm{i}}} \boldsymbol{W}_{\Gamma_{\mathrm{i}}}^{\mathrm{T}}\left(\boldsymbol{u}_{\Gamma_{\mathrm{i}}}^{+}-\boldsymbol{u}_{\Gamma_{\mathrm{i}}}^{-}\right) \mathrm{d} \Gamma=0
$$

Separando as parcelas da integração, utilizando a aproximação dada por (2.41) e (2.43) e sabendo que a igualdade acima é verdadeira para qualquer $\delta \boldsymbol{p}_{\Gamma_{\mathrm{i}}}^{\mathrm{T}}$, a equação resulta em:

$$
\begin{gathered}
\underbrace{\left[\int_{\Gamma_{i}} \boldsymbol{W}_{\Gamma_{i}}^{T} \boldsymbol{U}_{\Gamma_{i}}^{+} d \Gamma\right]}_{C_{\Gamma_{i}}^{+}} \boldsymbol{q}_{\Gamma_{i}}^{+}-\underbrace{\left[\int_{\Gamma_{i}} \boldsymbol{W}_{\Gamma_{i}}^{T} \boldsymbol{U}_{\Gamma_{i}}^{-} d \Gamma\right]}_{C_{\Gamma_{i}}^{-}} \boldsymbol{q}_{\Gamma_{i}}^{-}=0 \\
\boldsymbol{C}_{\Gamma_{\mathrm{i}}}^{+} \boldsymbol{q}_{\Gamma_{\mathrm{i}}}^{+}-\boldsymbol{C}_{\Gamma_{\mathrm{i}}}^{-} \boldsymbol{q}_{\Gamma_{\mathrm{i}}}^{-}=0
\end{gathered}
$$

Portanto, o sistema resolvente para a discretização com dois elementos é formado pela contribuição das equações de equilíbrio e compatibilidade no volume, equilíbrio nos contornos $\Gamma_{\sigma}$ e $\Gamma_{\mathrm{i}}$ e a relação de continuidade dos deslocamentos em $\Gamma_{\mathrm{i}}$. Tal sistema é representado por: 


$$
\left[\begin{array}{ccccccccc}
\boldsymbol{F}_{\Omega}^{1} & \boldsymbol{G}_{\Omega}^{1} & -\boldsymbol{G}_{\Gamma_{\sigma}}^{1} & -\boldsymbol{G}_{\Gamma_{\mathrm{i}}}^{+} & 0 & 0 & 0 & 0 & 0 \\
\boldsymbol{G}_{\Omega}^{1^{\mathrm{T}}} & 0 & 0 & 0 & 0 & 0 & 0 & 0 & 0 \\
-\boldsymbol{G}_{\Gamma_{\sigma}}^{1^{\mathrm{T}}} & 0 & 0 & 0 & 0 & 0 & 0 & 0 & 0 \\
-\boldsymbol{G}_{\Gamma_{\mathrm{i}}}^{+\mathrm{T}} & 0 & 0 & 0 & 0 & 0 & 0 & 0 & \boldsymbol{C}_{\Gamma_{\mathrm{i}}}^{+\mathrm{T}} \\
0 & 0 & 0 & 0 & \boldsymbol{F}_{\Omega}^{2} & \boldsymbol{G}_{\Omega}^{2} & -\boldsymbol{G}_{\Gamma_{\sigma}}^{2} & -\boldsymbol{G}_{\Gamma_{\mathrm{i}}}^{-} & 0 \\
0 & 0 & 0 & 0 & \boldsymbol{G}_{\Omega}^{2^{\mathrm{T}}} & 0 & 0 & 0 & 0 \\
0 & 0 & 0 & 0 & -\boldsymbol{G}_{\Gamma_{\sigma}}^{2^{\mathrm{T}}} & 0 & 0 & 0 & 0 \\
0 & 0 & 0 & 0 & -\boldsymbol{G}_{\Gamma_{\mathrm{i}}}^{-\mathrm{T}} & 0 & 0 & 0 & -\boldsymbol{C}_{\Gamma_{\mathrm{i}}}^{-\boldsymbol{T}} \\
0 & 0 & 0 & \boldsymbol{C}_{\Gamma_{\mathrm{i}}}^{+} & 0 & 0 & 0 & -\boldsymbol{C}_{\Gamma_{\mathrm{i}}}^{-} & 0
\end{array}\right]\left\{\begin{array}{c}
\boldsymbol{X}^{1} \\
\boldsymbol{q}_{\Omega}^{1} \\
\boldsymbol{q}_{\Gamma_{\sigma}}^{1} \\
\boldsymbol{q}_{\Gamma_{\boldsymbol{i}}}^{+} \\
\boldsymbol{X}^{2} \\
\boldsymbol{q}_{\Omega}^{2} \\
\boldsymbol{q}_{\Gamma_{\sigma}}^{2} \\
\boldsymbol{q}_{\Gamma_{\boldsymbol{i}}}^{-} \\
\boldsymbol{o}_{\Gamma_{\boldsymbol{i}}}
\end{array}\right\}=\left\{\begin{array}{c}
\boldsymbol{e}_{\Gamma_{\boldsymbol{u}}}^{1} \\
-\boldsymbol{Q}_{\Omega}^{1} \\
-\boldsymbol{Q}_{\Gamma_{\sigma}}^{1} \\
0 \\
\boldsymbol{e}_{\Gamma_{\boldsymbol{u}}}^{2} \\
-\boldsymbol{Q}_{\Omega}^{2} \\
-\boldsymbol{Q}_{\Gamma_{\sigma}}^{2} \\
0 \\
0
\end{array}\right\}
$$

A segunda hipótese consiste em impor a condição de continuidade dos deslocamentos na fronteira comum em forma forte (Equação (2.60)). Assim, a reciprocidade das forças passa a ser escrita em forma ponderada.

$$
\boldsymbol{u}_{\Gamma_{\mathrm{i}}}^{+}=\boldsymbol{u}_{\Gamma_{\mathrm{i}}}^{-}=\boldsymbol{u}_{\Gamma_{\mathrm{i}}}=\boldsymbol{U}_{\Gamma_{\mathrm{i}}} \boldsymbol{q}_{\Gamma_{\mathrm{i}}}
$$

A primeira equação do sistema linear passa a sofrer uma pequena modificação no termo associado a $\Gamma_{\mathrm{i}}$, já que os parâmetros referentes à aproximação dos deslocamentos nessa fronteira passam a ser iguais. As equações de compatibilidade de ambos os elementos em $\Gamma_{\mathrm{i}}$ passam a ser representadas por:

$$
\begin{aligned}
& \int_{\Gamma_{\mathrm{i}}^{+}}\left(\boldsymbol{I}_{+} \boldsymbol{S}_{\Omega}^{1}\right)^{\mathrm{T}} \boldsymbol{u}_{\Gamma_{\mathrm{i}}}^{+} \mathrm{d} \Gamma=\underbrace{\left[\int_{\Gamma_{\mathrm{i}}}\left(\boldsymbol{I}_{+} \boldsymbol{S}_{\Omega}^{1}\right)^{\mathrm{T}} \boldsymbol{U}_{\Gamma_{\mathrm{i}}} \mathrm{d} \Gamma\right]}_{\boldsymbol{G}_{\Gamma_{\mathrm{i}}}^{+}} \boldsymbol{q}_{\Gamma_{\mathrm{i}}}=\boldsymbol{G}_{\Gamma_{\mathrm{i}}}^{+} \boldsymbol{q}_{\Gamma_{\mathrm{i}}} \\
& \int_{\Gamma_{\overline{\mathrm{i}}}^{-}}\left(\boldsymbol{I}_{-} \boldsymbol{S}_{\Omega}^{2}\right)^{\mathrm{T}} \boldsymbol{u}_{\Gamma_{\mathrm{i}}}^{-} \mathrm{d} \Gamma=-\underbrace{\left[\int_{\Gamma_{\mathrm{i}}}\left(\boldsymbol{I}_{-} \boldsymbol{S}_{\Omega}^{2}\right)^{\mathrm{T}} \boldsymbol{U}_{\Gamma_{\mathrm{i}}} \mathrm{d} \Gamma\right]}_{\boldsymbol{G}_{\Gamma_{\mathrm{i}}}^{-}} \boldsymbol{q}_{\Gamma_{\Gamma_{\mathrm{i}}}}=-\boldsymbol{G}_{\Gamma_{\mathrm{i}}}^{-} \boldsymbol{q}_{\Gamma_{\mathrm{i}}}
\end{aligned}
$$

Já a reciprocidade é representada pela seguinte equação:

$$
\int_{\Gamma_{\mathrm{i}}} \delta \boldsymbol{u}_{\Gamma_{\mathrm{i}}}^{\mathrm{T}}\left(\boldsymbol{t}_{\Gamma_{\mathrm{i}}}^{+}+\boldsymbol{t}_{\Gamma_{\mathrm{i}}}^{-}\right) \mathrm{d} \Gamma=0
$$


Utilizando a aproximação $\delta \boldsymbol{u}_{\Gamma_{\mathrm{i}}}^{\mathrm{T}}=\delta \boldsymbol{q}_{\Gamma_{\mathrm{i}}}^{\mathrm{T}} \boldsymbol{U}_{\Gamma_{\mathrm{i}}}^{\mathrm{T}}$ na equação acima, separando os termos da integral e sabendo que a igualdade deve ser verdade para qualquer $\delta \boldsymbol{q}_{\Gamma_{\mathrm{i}}}^{\mathrm{T}}$, têm-se:

$$
\int_{\Gamma_{i}} U_{\Gamma_{i}}^{T} t_{\Gamma_{i}}^{+} d \Gamma+\int_{\Gamma_{i}} U_{\Gamma_{i}}^{T} t_{\Gamma_{i}}^{-} d \Gamma=0
$$

Uma vantagem dessa formulação é que a condição de equilíbrio do contorno $\Gamma_{\mathrm{i}}$ pode ser atendida utilizando o regime de tensões no volume de cada elemento (Equação (2.5)), reduzindo o número de incógnitas do sistema. Assim, a Equação (2.64) pode ser escrita como:

$$
\int_{\Gamma_{i}} \boldsymbol{U}_{\Gamma_{i}}^{T} \boldsymbol{I}_{+} \sigma_{\Omega}^{1} \mathrm{~d} \Gamma+\int_{\Gamma_{i}} \boldsymbol{U}_{\Gamma_{i}}^{T} \boldsymbol{I}_{-} \sigma_{\Omega}^{2} \mathrm{~d} \Gamma=0
$$

Utilizando a aproximação de tensão no domínio (Equação (2.14)) na equação acima, têm-se:

$$
\begin{gathered}
\underbrace{\left[\int_{\Gamma_{i}} \boldsymbol{U}_{\Gamma_{i}}^{T} \boldsymbol{I}_{+} \boldsymbol{S}_{\Omega}^{l} d \Gamma\right]}_{\boldsymbol{G}_{\Gamma_{i}}^{+T}} \boldsymbol{X}^{1}+\underbrace{\left[\int_{\Gamma_{i}} \boldsymbol{U}_{\Gamma_{i}}^{T} \boldsymbol{I}_{-} \boldsymbol{S}_{\Omega}^{2} d \Gamma\right]}_{\boldsymbol{G}_{\Gamma_{i}}^{-T}} \boldsymbol{X}^{2}=0 \\
\boldsymbol{G}_{\Gamma_{\mathrm{i}}^{+\mathrm{T}}}^{\boldsymbol{X}^{\mathrm{T}}} \boldsymbol{X}^{1}+\boldsymbol{G}_{\Gamma_{\Gamma_{\mathrm{i}}}^{-\mathrm{T}}}^{\boldsymbol{X}^{2}}=0
\end{gathered}
$$

Portanto, o sistema final fica composto pelas relações de compatibilidade e equilíbrio no volume, equilíbrio no contorno $\Gamma_{\sigma}$ de cada elemento mais a relação de reciprocidade de forças no contorno $\Gamma_{\mathrm{i}}$. Tal sistema é representado por:

$$
\left[\begin{array}{ccccccc}
\boldsymbol{F}_{\Omega}^{1} & \boldsymbol{G}_{\Omega}^{1} & -\boldsymbol{G}_{\Gamma_{\sigma}}^{1} & 0 & 0 & 0 & -\boldsymbol{G}_{\Gamma_{\mathrm{i}}}^{+} \\
\boldsymbol{G}_{\Omega}^{\mathbf{1}^{\boldsymbol{T}}} & 0 & 0 & 0 & 0 & 0 & 0 \\
-\boldsymbol{G}_{\Gamma_{\sigma}}^{\mathbf{T}^{\boldsymbol{T}}} & 0 & 0 & 0 & 0 & 0 & 0 \\
0 & 0 & 0 & \boldsymbol{F}_{\Omega}^{2} & \boldsymbol{G}_{\Omega}^{2} & -\boldsymbol{G}_{\Gamma_{\sigma}}^{2} & -\boldsymbol{G}_{\Gamma_{\mathrm{i}}}^{-} \\
0 & 0 & 0 & \boldsymbol{G}_{\Omega}^{2^{T}} & 0 & 0 & 0 \\
0 & 0 & 0 & -\boldsymbol{G}_{\Gamma_{\sigma}}^{2^{\boldsymbol{T}}} & 0 & 0 & 0 \\
-\boldsymbol{G}_{\Gamma_{\mathrm{i}}}^{+\boldsymbol{T}^{T}} & 0 & 0 & -\boldsymbol{G}_{\Gamma_{\mathrm{i}}}^{-\boldsymbol{T}} & 0 & 0 & 0
\end{array}\right]\left\{\begin{array}{c}
\boldsymbol{X}^{1} \\
\boldsymbol{q}_{\Omega}^{1} \\
\boldsymbol{q}_{\Gamma_{\sigma}}^{1} \\
\boldsymbol{X}^{2} \\
\boldsymbol{q}_{\Omega}^{2} \\
\boldsymbol{q}_{\Gamma_{\sigma}}^{2} \\
\boldsymbol{q}_{\Gamma_{\mathrm{i}}}
\end{array}\right\}=\left\{\begin{array}{c}
\boldsymbol{e}_{\Gamma_{\mathrm{u}}}^{1} \\
-\boldsymbol{Q}_{\Omega}^{1} \\
-\boldsymbol{Q}_{\Gamma_{\sigma}}^{1} \\
\boldsymbol{e}_{\Gamma_{\mathrm{u}}}^{2} \\
-\boldsymbol{Q}_{\Omega}^{2} \\
-\boldsymbol{Q}_{\Gamma_{\sigma}}^{2} \\
0
\end{array}\right\}
$$


Na primeira hipótese, foi admitida 'a priori' a reciprocidade das forças na fronteira comum aos elementos e a equação adicional foi a continuidade dos deslocamentos. Assim, a formulação passa a ser chamada de Híbrido-Mista de Deslocamento. Na segunda, a equação admitida 'a priori' foi a continuidade de deslocamentos, sendo a reciprocidade a equação adicional. Portanto, tal formulação é dita Híbrido-Mista de Tensão.

\section{5- Formulação híbrido}

A formulação híbrido é encontrada, basicamente, da mesma maneira que a híbridomista, com a diferença que agora a aproximação de tensões no domínio dos elementos deve obedecer a equação de equilíbrio em forma forte. Assim, substituindo a Equação (2.14) em (2.1) e desconsiderando as forças de volume, tem-se:

$$
L S_{\Omega}=0
$$

Com isso, a matriz $\boldsymbol{G}_{\Omega}$ se anula. Os sistemas para as formulações híbridos de deslocamento e híbridos de tensão assumem as seguintes formas, respectivamente:

$$
\begin{aligned}
& {\left[\begin{array}{ccccccc}
\boldsymbol{F}_{\Omega}^{1} & -\boldsymbol{G}_{\Gamma_{\sigma}}^{1} & -\boldsymbol{G}_{\Gamma_{\boldsymbol{i}}}^{+} & 0 & 0 & 0 & 0 \\
-\boldsymbol{G}_{\Gamma_{\sigma}}^{\mathbf{T}^{T}} & 0 & 0 & 0 & 0 & 0 & 0 \\
-\boldsymbol{G}_{\Gamma_{\boldsymbol{i}}}^{+\boldsymbol{T}} & 0 & 0 & 0 & 0 & 0 & \boldsymbol{C}_{\Gamma_{\boldsymbol{i}}}^{+\boldsymbol{T}} \\
0 & 0 & 0 & \boldsymbol{F}_{\Omega}^{2} & -\boldsymbol{G}_{\Gamma_{\sigma}}^{2} & -\boldsymbol{G}_{\Gamma_{\boldsymbol{i}}}^{-} & 0 \\
0 & 0 & 0 & -\boldsymbol{G}_{\Gamma_{\sigma}}^{2^{T}} & 0 & 0 & 0 \\
0 & 0 & 0 & -\boldsymbol{G}_{\Gamma_{\boldsymbol{i}}}^{-\boldsymbol{T}} & 0 & 0 & -\boldsymbol{C}_{\Gamma_{\boldsymbol{i}}}^{-\boldsymbol{T}} \\
0 & 0 & \boldsymbol{C}_{\Gamma_{\boldsymbol{i}}}^{+} & 0 & 0 & -\boldsymbol{C}_{\Gamma_{\boldsymbol{i}}}^{-} & 0
\end{array}\right]\left\{\begin{array}{c}
\boldsymbol{X}^{1} \\
\boldsymbol{q}_{\Gamma_{\sigma}}^{1} \\
\boldsymbol{q}_{\Gamma_{\boldsymbol{i}}}^{+} \\
\boldsymbol{X}^{2} \\
\boldsymbol{q}_{\Gamma_{\sigma}}^{2} \\
\boldsymbol{q}_{\Gamma_{\boldsymbol{i}}}^{-} \\
\boldsymbol{o}_{\Gamma_{\boldsymbol{i}}}
\end{array}\right\}=\left\{\begin{array}{c}
\boldsymbol{e}_{\Gamma_{\boldsymbol{u}}}^{1} \\
-\boldsymbol{Q}_{\Gamma_{\sigma}}^{1} \\
0 \\
\boldsymbol{e}_{\Gamma_{\boldsymbol{u}}}^{2} \\
\boldsymbol{Q}_{\Gamma_{\sigma}}^{2} \\
0 \\
0
\end{array}\right\}} \\
& {\left[\begin{array}{ccccc}
\boldsymbol{F}_{\Omega}^{1} & -\boldsymbol{G}_{\Gamma_{\sigma}}^{1} & 0 & 0 & -\boldsymbol{G}_{\Gamma_{\boldsymbol{i}}}^{+} \\
-\boldsymbol{G}_{\Gamma_{\sigma}}^{\mathbf{1}^{\boldsymbol{T}}} & 0 & 0 & 0 & 0 \\
0 & 0 & \boldsymbol{F}_{\Omega}^{2} & -\boldsymbol{G}_{\Gamma_{\sigma}}^{2} & -\boldsymbol{G}_{\Gamma_{\boldsymbol{i}}}^{-} \\
0 & 0 & -\boldsymbol{G}_{\Gamma_{\sigma}}^{2^{T}} & 0 & 0 \\
\boldsymbol{G}_{\Gamma_{\boldsymbol{i}}}^{+{ }^{T}} & 0 & -\boldsymbol{G}_{\Gamma_{\boldsymbol{i}}}^{-\boldsymbol{T}^{T}} & 0 & 0
\end{array}\right]\left\{\begin{array}{c}
\boldsymbol{X}^{1} \\
\boldsymbol{q}_{\Gamma_{\sigma}}^{1} \\
\boldsymbol{X}^{2} \\
\boldsymbol{q}_{\Gamma_{\sigma}}^{2} \\
\boldsymbol{q}_{\Gamma_{\boldsymbol{i}}}
\end{array}\right\}=\left\{\begin{array}{c}
\boldsymbol{e}_{\Gamma_{\boldsymbol{u}}}^{1} \\
-\boldsymbol{Q}_{\Gamma_{\sigma}}^{1} \\
\boldsymbol{e}_{\Gamma_{\boldsymbol{u}}}^{2} \\
-\boldsymbol{Q}_{\Gamma_{\sigma}}^{2} \\
0
\end{array}\right\}}
\end{aligned}
$$


Nota-se que na formulação híbrido apenas um campo é aproximado no domínio do elemento, no caso a tensão.

Mais informações sobre essas duas formulações podem ser encontradas no trabalho de Góis (2004) e Góis (2009). 


\section{3- Formulação híbrido-Trefftz de tensão}

\section{1- Considerações iniciais}

A formulação em estudo é baseada no Método de Trefftz, apresentada por Trefftz ${ }^{1}$ (1926 apud JIROUSEK; WRÓBLEWSKI, 1996). Esse método consiste em utilizar campos aproximados que atendam à equação diferencial governativa do problema, a equação de Navier (3.2).

O Método de Trefftz pode ser utilizado em combinação com qualquer método numérico. Os primeiros trabalhos sobre seu emprego no MEF foram realizados limitando sua aplicação em apenas partes do domínio do problema, sendo que no restante do domínio era utilizado o MEF clássico. Por exemplo, Stein² (1973 apud JIROUSEK; WRÓBLEWSKI, 1996) e Ruoff ${ }^{3}$ (1973 apud JIROUSEK; WRÓBLEWSKI, 1996) utilizaram a formulação em uma parte retangular de casca abatida.

Com o desenvolvimento computacional ao passar dos anos, a utilização desse método no âmbito do MEF passou a ganhar grande destaque. Jirousek e Leon (1977) foram os primeiros a formular um elemento baseado no Método de Trefftz que englobasse todo o domínio da estrutura. Eles apresentaram um elemento quadrilateral de placa fina com vinte e quatro graus de liberdade. Jirousek (1978) desenvolveu quatro elementos híbrido-Trefftz cuja aplicação também envolvia todo o domínio do elemento. Desses quatro, um elemento se destacou sobre os demais: o HT-D (“hybrid Trefftz displacement”). Jirousek e Teodorescu (1982) aplicaram a formulação em problemas da elasticidade plana e placas de Kirchhoff, mencionando esses elementos como "large finite elements" (LFE). Jirousek e Venkatesh (1992) analisaram o desempenho da formulação em diversos problemas da elasticidade plana.

\footnotetext{
${ }^{1}$ TREFFTZ, E. (1926). Ein Gegenstück zum Ritzschen Verfahren. Procedures 2nd International Congress of Applied Mechanics, Zurich, p. 131-137.

2 STEIN, E. (1973). Die Kombination des modifizierten Trefftzschen Verfahrens mit der Methode der Finiten Elemente. Finite Elemente in der Statik (K. Buch, D. Scharpf, E. Stein, and W. Wunderlich, eds.), p.172-185.

${ }^{3}$ RUOFF, G. (1973). Die praktische Berechnung der Kopplungsmatrizen bei der Kombination der Trefftzschen Metode und der Metode der finiten Elemente beu flachen Schalen. Finite Elemente in der Statik (K. Buch, D. Scharpf, E. Stein, and W. Wunderlich, eds.), p. 242-259.
} 
Como se pode observar, Jirousek foi um grande estudioso dessa formulação, trazendo vários avanços para essa área. Além dele, outros pesquisadores utilizaram essa formulação em seus trabalhos como, por exemplo, Petrolito (1996) que aplicou a formulação em placas espessas e Freitas (1998) apresentou o elemento HTS ("hybrid Trefftz stress"). Este elemento possuía uma descontinuidade nos campos de deslocamentos no contorno estático do elemento, mais especificamente nas suas quinas. Segundo o autor, tal descontinuidade proporcionava uma melhor aproximação dos campos de tensão. Ele também mostrou que apesar do nome, o elemento HT-D de Jirousek é um elemento de tensão.

Ao garantir que os campos de tensões atendam à equação de Navier, todas as integrais do sistema ficam definidas apenas no contorno do elemento, proporcionando ao elemento finito essa grande vantagem, característica do Método dos Elementos de Contorno (MEC). Com isso, a aproximação do problema não apresenta sensibilidade à distorção da rede e abre a possibilidade da utilização de elementos com diversos tipos de geometria.

Em torno disso, portanto, a restrição de atendimento da equação de Navier pelos campos de tensão faz com que essa formulação apresente melhores resultados em relação às outras formulações não-convencionais em problemas da elasticidade linear.

\section{2- Elemento finito híbrido-Trefftz de tensão}

Neste trabalho, foi adotado o elemento finito híbrido-Trefftz quadrilateral de quatro nós, o mesmo apresentado por Souza (2008), representado pela Figura 3.1. Tal elemento é formulado baseado no modelo de tensão (equilíbrio nos contornos internos expresso em forma fraca), comentado no capítulo anterior.

Os campos de tensões são aproximados no domínio do elemento utilizando coordenadas cartesianas com origem no seu centróide. Já no contorno do elemento, os campos de deslocamentos são aproximados de forma independente utilizando um sistema de coordenadas adimensionais para cada lado com origem na respectiva mediana. 


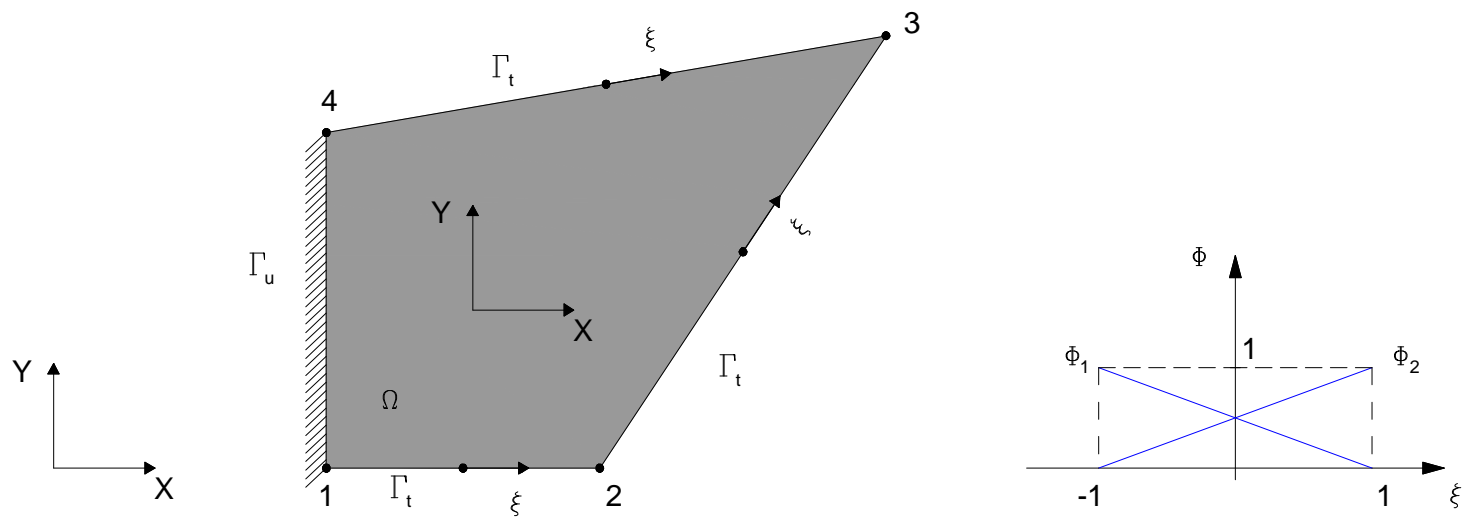

Figura 3.1 - Elemento finito híbrido-Trefftz.

\subsection{1- Equação de Navier}

Para deduzir as equações da formulação híbrido-Trefftz de tensão é necessário, inicialmente, conhecer a equação diferencial que governa o problema elástico linear: a equação de Navier.

Uma vez conhecidas as equações de equilíbrio, compatibilidade e constitutiva da elasticidade, apresentadas no item anterior, deduz-se a equação de Navier pelos seguintes passos: inicialmente substitui-se a Equação (2.2) em (2.4):

$$
\sigma=D L^{T} u
$$

Em seguida, é feita a substituição da Equação (3.1) na (2.1), obtendo-se a equação de Navier:

$$
\boldsymbol{L}\left(\boldsymbol{D} \boldsymbol{L}^{T} u\right)=-b
$$

Podemos simplificá-la pela seguinte representação:

$$
\mathfrak{R}(\boldsymbol{u})=-\boldsymbol{b}
$$

Onde $\mathfrak{R}$ é um operador matricial. 


\subsection{2- Aproximação do campo de deslocamentos no domínio do elemento atendendo a equação de Navier}

Para empregar o Método de Trefftz deve-se encontrar os campos de deslocamento que atendam a equação de Navier. As bases da aproximação de tensão no domínio serão derivadas das bases encontradas para o deslocamento no domínio

Assim, dada a relação (3.3), pode-se aproximar o campo de deslocamentos por duas parcelas: uma solução particular $\hat{\boldsymbol{u}}$ e uma solução homogênea $\boldsymbol{u}_{\boldsymbol{h}}$. Essa aproximação é dada por:

$$
\boldsymbol{u}=\hat{\boldsymbol{u}}+\boldsymbol{u}_{h}, \text { em } \Omega
$$

Matematicamente falando, a solução particular $\hat{\boldsymbol{u}}$ é a solução da equação diferencial quando a mesma não é homogênea, como, por exemplo, ao adotar $\boldsymbol{b} \neq 0$. Portanto, representa-se essa solução por:

$$
\Re(\hat{\boldsymbol{u}})=-\boldsymbol{b}, \text { em } \Omega
$$

onde:

$$
\hat{\boldsymbol{u}}^{\boldsymbol{T}}=\left\{\begin{array}{ll}
\hat{u}_{x} & \hat{u}_{y}
\end{array}\right\}
$$

Sendo $\hat{u}_{x}$ e $\hat{u}_{y}$ componentes da solução particular.

Já a solução homogênea diz respeito à equação diferencial escrita na forma:

$$
\Re\left(\boldsymbol{u}_{\boldsymbol{h}}\right)=0, \text { em } \Omega
$$

Pode-se aproximar a solução homogênea por uma combinação linear:

$$
u_{h}=\sum_{i=1}^{n} c_{i} N_{i}=N c
$$

Na solução anterior $c$ é o vetor de parâmetros da combinação linear, representado por:

$$
c^{T}=\left\{\mathrm{c}_{1} \cdots \mathrm{c}_{\mathrm{n}}\right\}
$$


e a matriz $N$ é constituída pelos vetores $N_{i}$ que representam as bases de aproximação da solução. Ela é dada por:

$$
N=\left[N_{1} \cdots N_{n}\right]
$$

onde:

$$
N_{i}^{T}=\left[\begin{array}{ll}
\mathrm{N}_{\mathrm{ix}} & \mathrm{N}_{\mathrm{iy}}
\end{array}\right], \text { para } \mathrm{i}=1, \ldots, \mathrm{n}
$$

Cada vetor $N_{i}$ é solução da homogênea, isto é:

$$
\Re\left(N_{i}\right)=0, \text { em } \Omega
$$

já que a Equação (3.7) é verdadeira para qualquer valor de $\boldsymbol{c}_{\boldsymbol{i}}$.

Unindo as Equações (3.4) e (3.8), obtemos a expressão para a aproximação do campo de deslocamentos no domínio do elemento:

$$
u=\hat{u}+u_{h}=\hat{u}+\sum_{i=1}^{n} c_{i} N_{i}=\hat{u}+N c, \text { em } \Omega
$$

sendo $\boldsymbol{c}_{i}$ são os parâmetros a serem determinados. Eles podem ser obtidos utilizando qualquer método numérico. Segundo Jirousek e Wróblewski (1996), a solução converge para a solução exata à medida que o número de termos da aproximação aumenta.

Neste trabalho, desconsideram-se as forças volúmicas, tornando a solução particular igual a zero $(\hat{\boldsymbol{u}}=0)$. Consequentemente, a aproximação do campo de deslocamento passa a ser escrita como:

$$
u=u_{h}=\sum_{i=1}^{n} c_{i} N_{i}=N c, \text { em } \Omega
$$

Tendo em mãos a aproximação do deslocamento no domínio do elemento, resta agora encontrar as funções $N_{i}$. 


\subsubsection{1- Solução Homogênea}

Como já foi comentado, o campo de aproximação dos deslocamentos no domínio do elemento deve atender a equação de Navier. Para isso, o conjunto de vetores $\boldsymbol{N}_{\boldsymbol{i}}$ deve atender a Equação (3.12).

A solução da Equação (3.12) para o estado plano de tensões (EPT) foi proposta por Muskhelishvili (1953 apud JIROUSEK; VENKATESH, 1992) utilizando variáveis complexas. Assim, as componentes $\boldsymbol{u}_{\boldsymbol{x}}$ e $\boldsymbol{u}_{\boldsymbol{y}}$ do campo dos deslocamentos devem atender à seguinte equação:

$$
E\left(u_{x}+i u_{y}\right)=(3-v) F(z)-(1+v)\left[z \overline{F^{\prime}(z)}+\overline{G(z)}\right]
$$

onde $E$ é o módulo de elasticidade do material, $v$ o coeficiente de Poisson, $F(z)$ e $G(z)$ são pares de funções arbitrárias, $z=x+i y$ é um número complexo, composto por uma parte real $(\operatorname{Re}(z)=x)$ e uma parte imaginária $(\operatorname{Im}(z)=y)$. A notação $i$ representa a unidade imaginária, dada por $i=\sqrt{-1} \cdot \bar{z}=x-i y$ é o conjugado de $z, \overline{G(z)}$ representa o conjugado de $G(\bar{z})$ e $\overline{F^{\prime}(z)}$ é o conjugado de $F^{\prime}(\bar{z})$, sendo $F^{\prime}(z)=\frac{d F(z)}{d z}$.

Podem-se obter infinitas funções de $u_{x}$ e $u_{y}$ a depender da escolha de $F(z)$ e $G(z)$. Nesse trabalho adota-se o conjunto de funções polinomiais utilizados no trabalho de Jirousek e Venkatesh (1992), para definir os seguintes pares de funções $F(z)$ e $G(z)$ :

$$
\begin{aligned}
& F(z)=i z^{k} \text { e } G(z)=0 \\
& F(z)=z^{k} \text { e } G(z)=0 \\
& F(z)=0 \text { e } G(z)=i z^{k} \\
& F(z)=0 \text { e } G(z)=z^{k}
\end{aligned}
$$

onde $\mathrm{k}=1, \ldots, \mathrm{n}$. 
Cada par de funções acima (com $\mathrm{k}=1, \ldots, \mathrm{n})$ gera uma base de vetores $\boldsymbol{N}_{\boldsymbol{i}}$ linearmente independentes. A base se completa pela união de todos os vetores $\boldsymbol{N}_{\boldsymbol{i}}$ formados a partir dos pares de funções dadas por (3.16), (3.17), (3.18) e (3.19).

Para esclarecer o procedimento de obtenção das bases $N_{i}$, considere o par de funções dadas por (3.16), onde, para esse par, $F^{\prime}(z)=i k z^{k-1}, \overline{F^{\prime}(z)}=-i k \bar{z}^{k-1}$ e $\overline{G(z)}=0$. Substituindo essas relações em (3.15), obtêm-se a seguinte expressão:

$$
E\left(u_{x}+i u_{y}\right)=(3-v) i z^{k}+(1+v)\left(k i z \bar{z}^{k-1}\right)
$$

nomeando o termo da direita como $A_{k}=(3-v) i z^{k}+(1+v)\left(k i z \bar{z}^{k-1}\right)$, identificam-se: $u_{x}=\frac{\operatorname{Re}\left(A_{k}\right)}{E}$ e $u_{y}=\frac{\operatorname{Im}\left(A_{k}\right)}{E}$, sendo $u_{x}$ e $u_{y}$ as componentes de $N_{A k}$.

A base total de vetores da aproximação é dada por:

$$
\begin{gathered}
N_{A k}=\frac{1}{E}\left\{\begin{array}{l}
\operatorname{Re}\left(A_{k}\right) \\
\operatorname{Im}\left(A_{k}\right)
\end{array}\right\}, \text { sendo } A_{k}=(3-v) i z^{k}+(1+v)\left(k i z \bar{z}^{k-1}\right) \\
N_{B k}=\frac{1}{E}\left\{\begin{array}{l}
\operatorname{Re}\left(B_{k}\right) \\
\operatorname{Im}\left(B_{k}\right)
\end{array}\right\} \text {, sendo } B_{k}=(3-v) z^{k}-(1+v)\left(k z \bar{z}^{k-1}\right) \\
N_{C k}=\frac{1}{E}\left\{\begin{array}{l}
\operatorname{Re}\left(C_{k}\right) \\
\operatorname{Im}\left(C_{k}\right)
\end{array}\right\}, \text { sendo } C_{k}=(1+v) i \bar{z}^{k} \\
N_{D k}=\frac{1}{E}\left\{\begin{array}{l}
\operatorname{Re}\left(D_{k}\right) \\
\operatorname{Im}\left(D_{k}\right)
\end{array}\right\}, \text { sendo } D_{k}=-(1+v) \bar{z}^{k}
\end{gathered}
$$

onde $k=1, \ldots, \mathrm{n}$.

Ao substituir $k=1$ em (3.21), obtemos as seguintes expressões abaixo:

$$
\begin{aligned}
& u_{x}=-4 E^{-1} y \\
& u_{y}=4 E^{-1} x
\end{aligned}
$$

que estão associadas a giro de corpo rígido dados por: 


$$
\omega=\frac{1}{2}\left(\frac{\partial u_{x}}{\partial y}-\frac{\partial u_{y}}{\partial x}\right)=-4 E^{-1}
$$

Portanto, ao utilizar a base de vetores $N_{i}$, deve-se excluir a base $N_{A 1}$, pois ela representa os movimentos de corpo rígido do sólido.

Assim, o número $n$ de vetores $\boldsymbol{N}_{i}$ e de coeficientes $\boldsymbol{c}_{i}$ da Equação (3.8), é dado por $n=4 k-1$. Portanto, pode-se representar $N$ por:

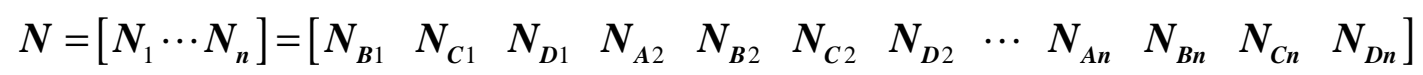

Ou, utilizando as relações (3.21), (3.22), (3.23) e (3.24), representa-se $N$ por:

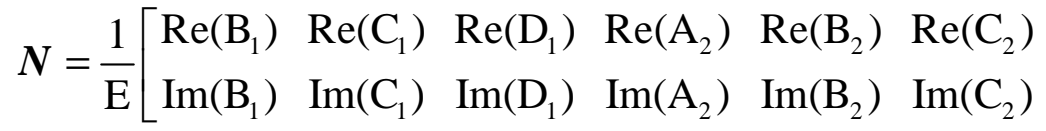

$$
\begin{aligned}
& \operatorname{Re}\left(\mathrm{D}_{2}\right) \quad \operatorname{Re}\left(\mathrm{A}_{\mathrm{n}}\right) \operatorname{Re}\left(\mathrm{B}_{\mathrm{n}}\right) \operatorname{Re}\left(\mathrm{C}_{\mathrm{n}}\right) \operatorname{Re}\left(\mathrm{D}_{\mathrm{n}}\right) \\
& \operatorname{Im}\left(D_{2}\right){ }^{\cdots} \operatorname{Im}\left(A_{n}\right) \quad \operatorname{Im}\left(B_{n}\right) \quad \operatorname{Im}\left(C_{n}\right) \quad \operatorname{Im}\left(D_{n}\right)
\end{aligned}
$$

No anexo (A), retirado do trabalho de Souza (2008), encontra-se a extensão da matriz $N$ até a $47^{\mathrm{a}}$ coluna.

\subsection{3- Aproximação do campo de tensões no domínio}

A aproximação do campo de tensões no domínio do elemento é obtida a partir da aproximação do campo de deslocamento do domínio encontrada no capítulo anterior. Para isso, deve-se utilizar a equação de compatibilidade e a constitutiva (Equações (2.2) e (2.4), respectivamente). Assim, obtêm-se:

$$
\sigma=\sum_{i=1}^{n} c_{i} S_{i}=S c \quad, \text { em } \Omega
$$

onde $S_{i}=D L^{T} N_{i}$ e $S=D L^{T} N$ 
O vetor $\boldsymbol{c}$ coleta os parâmetros $c_{i}$ da combinação linear. Vale destacar que eles não representam graus de liberdade, portanto deve-se realizar um pós-processamento para encontrar o valor das tensões nos pontos desejados.

A matriz $S$ coleta as funções de aproximação $S_{i}$ obtidas a partir de $N_{i}$.

No anexo (A), retirado do trabalho de Souza (2008), encontra-se a extensão da matriz $S$ até a $47^{\text {a }}$ coluna.

\subsection{4- Aproximação do campo de tensões no contorno}

A aproximação do campo de tensões no contorno do elemento é obtida da aproximação do campo de tensões no domínio pelo teorema de Cauchy. Assim, utilizando a Equação (3.29) e em seguida a (2.5), temos:

$$
\boldsymbol{t}=\boldsymbol{I} \boldsymbol{\sigma}=\sum_{i=1}^{n} c \boldsymbol{T}_{i}=\boldsymbol{T} \boldsymbol{c}, \mathrm{em} \Gamma
$$

onde $\boldsymbol{T}_{i}=\boldsymbol{I} \boldsymbol{S}_{i}$ e $\boldsymbol{T}=\boldsymbol{I S}$

\subsection{5- Aproximação do campo de deslocamentos no contorno}

A cada lado do elemento é atrelada uma aproximação independente, tendo como referência o sistema de coordenadas adimensionais com origem em cada mediana (ver Figura 3.1). Os deslocamentos do lado coincidente com o contorno cinemático do sólido são conhecidos e impostos em forma forte.

A equação que define a aproximação dos deslocamentos é representada por:

$$
\tilde{\boldsymbol{u}}=\widetilde{\boldsymbol{U}} \boldsymbol{d}, \operatorname{em} \Gamma_{\sigma}
$$

Sendo $\tilde{\boldsymbol{u}}$ a aproximação do campo dos deslocamentos, $\widetilde{\boldsymbol{U}}$ a matriz contendo as funções de forma e $\boldsymbol{d}$ o vetor que contém os graus de liberdade em deslocamentos. 
Para ilustrar, considere-se o trecho que contém os nós 1 e 2 da Figura 3.1. A Equação (3.31) referente a esse trecho é escrita da seguinte forma:

$$
\left\{\begin{array}{l}
u_{x} \\
u_{y}
\end{array}\right\}=\left[\begin{array}{llll}
\phi_{1} & 0 & \phi_{2} & 0 \\
0 & \phi_{1} & 0 & \phi_{2}
\end{array}\right]\left\{\begin{array}{l}
u_{x 1} \\
u_{y 1} \\
u_{x 2} \\
u_{y 2}
\end{array}\right\}
$$

onde:

- $u_{x}$ e $u_{y}$ são as componentes do vetor de deslocamento $\tilde{\boldsymbol{u}}$;

- $\quad \phi_{1}$ e $\phi_{2}$ são as funções de forma escritas em coordenadas adimensionais. Elas valem:

$$
\left\{\begin{array}{l}
\phi_{1}=\frac{1}{2}(-\xi+1) \\
\phi_{2}=\frac{1}{2}(\xi+1)
\end{array}\right.
$$

- $\quad u_{x 1}$ e $u_{y 1}$ são os graus de liberdade em x e y do nó 1 , respectivamente.

- $u_{x 2}$ e $u_{x 2}$ são os graus de liberdade em x e y do nó 2, respectivamente.

\subsection{6- Equacionamento}

O equacionamento da formulação híbrido-Trefftz segue a mesma sequência da formulação híbrido; a diferença é que agora a restrição imposta à aproximação de tensão no domínio exige o atendimento à equação de Navier.

Assim, substituindo a equação de compatibilidade (2.2) e a relação constitutiva (2.4) na Equação (3.2) e desprezando as forças volúmicas, tem-se:

$$
\boldsymbol{L}\left(\boldsymbol{D} \boldsymbol{L}^{T} \boldsymbol{u}\right)=\boldsymbol{L}(\boldsymbol{D} \boldsymbol{\varepsilon})=\boldsymbol{L}(\boldsymbol{\sigma})=\mathbf{0}, \mathrm{em} \boldsymbol{\Omega}
$$

Substituindo a aproximação (3.29) e sabendo que a igualdade acima é válida para qualquer valor do parâmetro $c_{i}$, tem-se que:

$$
\boldsymbol{L} \boldsymbol{S}=\mathbf{0}, \text { em } \boldsymbol{\Omega}
$$


Assim, substituindo a equação acima no sistema linear (2.24), observa-se que a matriz $\boldsymbol{G}_{\boldsymbol{\Omega}}$ torna-se trivial. Como não há aproximação do campo de deslocamentos no domínio do elemento, os índices subscritos $\Omega$ e $\Gamma_{\sigma}$ serão omitidos.

O sistema linear fica representado por:

$$
\left[\begin{array}{cc}
F & -G \\
-G^{T} & 0
\end{array}\right]\left\{\begin{array}{l}
c \\
d
\end{array}\right\}=\left\{\begin{array}{c}
e \\
-q
\end{array}\right\}
$$

No sistema acima, os vetores $\boldsymbol{e}$ e $\boldsymbol{q}$ são os mesmos que as variáveis definidas em (2.28) e (2.30), respectivamente.

Substituindo a aproximação de tensões no contorno do elemento $\boldsymbol{T}=\boldsymbol{I} \boldsymbol{S}$ em (2.27), a matriz $\boldsymbol{G}$ fica representada por:

$$
\boldsymbol{G}=\int_{\Gamma} T^{T} \widetilde{U} \mathrm{~d} \Gamma
$$

Como os campos de tensão no domínio do elemento são auto-equilibrados, pode-se representar a matriz $\boldsymbol{F}$ por uma integral no contorno do elemento. Isso pode ser verificado pelo seguinte procedimento: dado a expressão de $\boldsymbol{F}$ em (2.25), sabendo que $\boldsymbol{S}=\boldsymbol{D} \boldsymbol{L}^{\boldsymbol{T}} \boldsymbol{N}$ e que a matriz de flexibilidade $f$ é a inversa da matriz de rigidez $\boldsymbol{D}$, tem-se:

$$
F=\int_{\Omega} S^{T} f S \mathrm{~d} \Omega=\int_{\Omega} S^{T} f D L^{T} N \mathrm{~d} \Omega=\int_{\Omega} S^{T} L^{T} N \mathrm{~d} \Omega
$$

Integrando a equação acima por partes, obtêm-se:

$$
\boldsymbol{F}=\int_{\Omega} \boldsymbol{S}^{T} \boldsymbol{L}^{T} \boldsymbol{N d} \Omega=-\int_{\Omega}(\boldsymbol{L S})^{T} \boldsymbol{N d} \Omega+\int_{\Gamma}(\boldsymbol{I S})^{T} \boldsymbol{N} \mathrm{d} \Gamma
$$

Pela condição (3.35) e como a aproximação de tensões no contorno é dada por $\boldsymbol{T}=\boldsymbol{I} \boldsymbol{S}$, a Equação (3.39) passa a ser:

$$
\boldsymbol{F}=\int_{\Gamma} \boldsymbol{T}^{\boldsymbol{T}} \boldsymbol{N d} \Gamma
$$


Vale observar que a matriz $\boldsymbol{F}$ é formada pelo produto de matrizes que possuem colunas linearmente independentes. Segundo Freitas (1998) essa condição garante que a matriz seja não-singular e positiva definida.

A condição necessária e suficiente para a existência e unicidade da solução para formulações mistas foi tratada por Zienkiewicz et al. (1986). Abaixo se descreve esse mesmo estudo para a formulação híbrido-Trefftz.

Inicialmente, considere-se a matriz $\boldsymbol{F}$ de dimensões $\left(\mathrm{n}_{\mathrm{c}} \times \mathrm{n}_{\mathrm{c}}\right)$, a matriz $\boldsymbol{G}$ de dimensões $\left(\mathrm{n}_{\mathrm{c}} \times \mathrm{n}_{\mathrm{d}}\right)$, os vetores $\boldsymbol{c}$ e $\boldsymbol{e}$ de dimensões $\left(\mathrm{n}_{\mathrm{c}}\right)$ e os vetores $\boldsymbol{d}$ e $\boldsymbol{q}$ de dimensões $\left(\mathrm{n}_{\mathrm{d}}\right)$.

Levando em conta a primeira equação do sistema (3.36), pode-se obter o vetor $\boldsymbol{c}$ :

$$
c=F^{-1} G d+F^{-1} e
$$

sendo $\boldsymbol{F}$ uma matriz não singular.

Substituindo a Equação (3.41) na segunda equação do sistema (3.36), obtém-se:

$$
\begin{gathered}
\underbrace{G^{T} F^{-1} G}_{K_{H T}} d+G^{T} F^{-1} e=q \\
K_{H T} d=q-G^{T} F^{-1} e
\end{gathered}
$$

Percebe-se que a existência e unicidade do sistema dependem da matriz $\boldsymbol{K}_{H T}$ definida acima. A fim de analisar as dimensões das matrizes, considere-se que:

$$
\boldsymbol{K}_{\boldsymbol{H} \boldsymbol{T}}=\boldsymbol{B}_{\left(\mathrm{n}_{\mathrm{d}} \times \mathrm{n}_{\mathrm{c}}\right)} \boldsymbol{G}_{\left(\mathrm{n}_{\mathrm{c}} \times \mathrm{n}_{\mathrm{d}}\right)}
$$

Sendo $B=G^{T} F^{-1}$

Para que a matriz $\boldsymbol{K}_{\boldsymbol{H} T}$ não seja singular, o número de colunas da matriz $\boldsymbol{B}$ não deve ser inferior ao número de colunas da matriz $\boldsymbol{G}$ (HOFFMAN, K.; KUNZER, R., 1971). Algebricamente, a condição necessária para a solução do sistema (3.36) é dada por:

$$
\mathrm{n}_{\mathrm{c}} \geq \mathrm{n}_{\mathrm{d}}
$$

Portanto o número de parâmetros de tensão não deve ser menor que o número de graus de liberdade em deslocamento. 
A condição suficiente é obtida com a positividade da matriz $\boldsymbol{K}_{\boldsymbol{H T}}$. Para isso, a seguinte condição tem que ser atendida:

$$
d^{T} K_{H T} d>0, \forall d \neq 0
$$

Mas como $\boldsymbol{K}_{\boldsymbol{H}}=\boldsymbol{G}^{T} \boldsymbol{F}^{-1} \boldsymbol{G}$, e $\boldsymbol{F}$ é uma matriz positiva definida, então a condição acima é atendida se:

$$
\boldsymbol{G d}>0, \forall \boldsymbol{d} \neq 0
$$

Escrevendo a equação acima segundo uma transformação linear, temos que:

$$
\mathrm{T}: \mathbb{R}^{\mathrm{n}_{\mathrm{d}}} \rightarrow \mathbb{R}^{\mathrm{n}_{\mathrm{c}}}, \text { tal que } \mathrm{T}(\boldsymbol{d})=\boldsymbol{G} \boldsymbol{d} \neq \mathbf{0}, \forall \boldsymbol{d} \neq 0
$$

Para que a Equação (3.48) seja satisfeita, a transformação linear deve ser injetora (HOFFMAN, K.; KUNZER, R., 1971). Assim, ela deve atender as seguintes condições:

- $\mathrm{n}_{\mathrm{c}} \geq \mathrm{n}_{\mathrm{d}}$;

- Posto (número de colunas linearmente independentes) de $\boldsymbol{G}$ igual a $\mathrm{n}_{\mathrm{d}}$.

Como $\boldsymbol{G}$ possui dimensões $\left(\mathrm{n}_{\mathrm{c}} \times \mathrm{n}_{\mathrm{d}}\right)$ ambas as condições são atendidas. Percebe-se que o atendimento da primeira condição já garante o atendimento da segunda, pois as matrizes envolvidas para o cálculo de $\boldsymbol{G}$ apresentam colunas linearmente independentes.

Portanto, para a formulação híbrido-Trefftz, apenas o atendimento da equação de estabilidade já garante a existência e unicidade da solução da Equação (3.36).

\subsection{7- Montagem do sistema global}

O sistema dado pela Equação (3.36) é aplicado apenas a um elemento finito. Para utilizar uma rede de elementos finitos deve-se montar o sistema resolutivo levando em conta as mesmas condições para as formulações apresentadas no capítulo anterior. 
Como o elemento do presente trabalho é baseado no modelo de tensão, deve-se considerar na fronteira comum aos elementos vizinhos $\left(\Gamma_{\boldsymbol{i}}\right)$ a continuidade dos deslocamentos em forma forte e o equilíbrio das forças nodais equivalentes em média.

Assim, a condição de continuidade dos deslocamentos em $\Gamma_{i}$ vale:

$$
\tilde{u}^{i}=\tilde{\boldsymbol{u}}^{j}
$$

Onde $i$ e $j$ representam elementos com uma fronteira em comum.

Já a condição do equilíbrio das forças nodais equivalentes num nó $k$ pertencente a um lado $l$ do elemento finito vale:

$$
\sum \boldsymbol{q}_{\sigma k l}=\sum \boldsymbol{q}_{\bar{t} k l}
$$

Onde $\boldsymbol{q}_{\sigma k l}$ representa o vetor de forças nodais equivalentes provenientes de tensão e $\boldsymbol{q}_{\bar{t} k l} \mathrm{O}$ vetor de forças nodais equivalentes provenientes do carregamento.

Para ilustrar a montagem do sistema global, considere-se uma rede contendo $n$ elementos finitos com um total de $n_{t}$ graus de liberdade em deslocamento. Assim, o sistema é representado por:

$$
\left(\begin{array}{ccccc}
F_{1} & 0 & & 0 & \overbrace{-G_{1}}^{1,2, \ldots, n_{t}} \\
0 & F_{2} & \cdots & 0 & -G_{2} \\
& & \ddots & \vdots \\
0 & 0 & & F_{n} & -G_{n} \\
-G_{1}^{T} & -G_{2}^{T} & & -G_{n}^{T} & 0
\end{array}\right)\left\{\begin{array}{c}
c_{1} \\
c_{2} \\
\vdots \\
c_{n} \\
d_{t}
\end{array}\right\}=\left\{\begin{array}{c}
e_{1} \\
e_{2} \\
\vdots \\
e_{n} \\
-q_{t}
\end{array}\right\}
$$

onde o vetor $\boldsymbol{d}_{\boldsymbol{t}}$ representa os $n_{t}$ graus de liberdade em deslocamento e o vetor $\boldsymbol{q}_{\boldsymbol{t}}$ pode ser interpretado como um vetor de forças nodais equivalentes.

A condição de compatibilidade de deslocamentos, dada por (3.49), e o equilíbrio das forças, dado por (3.50), é atendido através da alocação de cada matriz $\boldsymbol{G}$, onde sua posição na linha depende de cada elemento, e do vetor $\boldsymbol{q}_{\boldsymbol{t}}$ pelo compartilhamento dos graus de liberdade nodais entre elementos vizinhos. 
Um esboço mais detalhado da montagem do sistema global pode ser encontrado no trabalho de SOUZA (2008).

A existência e unicidade da solução do sistema global ainda estão atreladas à condição de estabilidade dada por (3.45). Porém, essa condição pode ser relaxada devido ao compartilhamento de graus de liberdade em deslocamento entre elementos vizinhos. Assim, a Equação (3.45) deve ser verificada para cada conjunto possível de elementos da rede. Esse conjunto é denominado de "mosaico" (ZIENKIEWICZ et al., 1986). Vale destacar que o não atendimento dessa condição num único "mosaico" pode comprometer a estabilidade da solução mesmo que ela seja atendida na totalidade dos graus de liberdade da rede.

\section{3- Elemento híbrido-Trefftz de tensão com enriquecimento seletivo}

\subsection{1- Considerações iniciais}

Como comentado, as aproximações dos deslocamentos no contorno do elemento são feitas mediante bases de funções lineares. Essas bases causam um empobrecimento na solução final ao utilizar uma rede pouco refinada, já que não leva em conta a distribuição dos vetores de forma diferente da linear. Além do grau, a quantidade de funções utilizadas, apenas duas, também pode exigir um refinamento excessivo da rede a fim de proporcionar uma solução confiável.

Assim, neste trabalho, utilizam-se as técnicas de enriquecimento no contorno do elemento híbrido-Trefftz, propostas por SOUZA (2008), com a finalidade de promover uma melhora na aproximação.

O enriquecimento realizado nesse elemento consiste em utilizar o clássico refino-p, onde serão adicionadas novas bases de aproximação no campo de deslocamento do contorno do elemento. Para aprimorar o enriquecimento, faz-se recurso ao enriquecimento seletivo, inspirando-se no MEFG. O enriquecimento não é realizado em todos os elementos da rede e sim em certos elementos que possuem um nó em comum. Essa região é denominada de nuvem (Figura 3.2). 


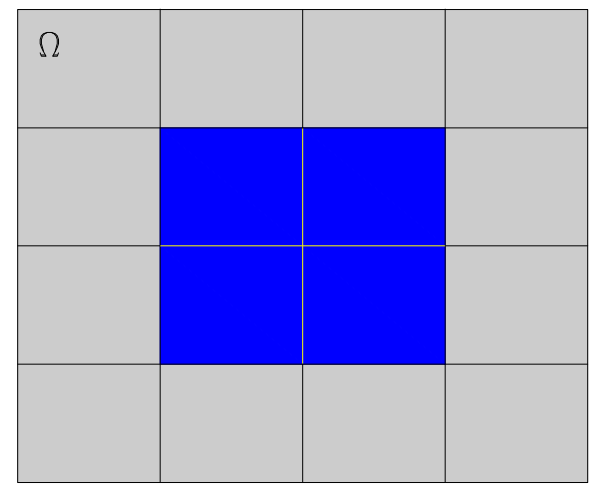

Trechos enriquecidos

Nuvem

Figura 3.2 - Nuvem do enriquecimento seletivo

Como cada trecho do elemento é aproximado de forma independente dos demais, eles também serão enriquecidos de forma independente.

A adição de novas bases de aproximação gera novos graus de liberdade em deslocamento. Eles não apresentam qualquer significado físico, mas fazem parte do sistema resolutivo do problema. Esses novos graus de liberdade devem ser levados em conta no teste do "mosaico", portanto pode ser necessária a adição de novos graus de liberdade em tensão.

Neste trabalho, foram utilizadas três bases de aproximações: uma base polinomial hierárquica, uma base polinomial não-hierárquica e uma base trigonométrica. Elas serão apresentadas a seguir.

\subsection{2- Bases enriquecedoras}

Para garantir uma boa solução final, as bases enriquecedoras devem possuir boas características de aproximação do problema. Além disso, é necessário que elas sejam fruto de uma partição da unidade (PU), já que isso garantirá a continuidade dos vetores de deslocamento entre os trechos dos elementos.

Como já foi comentada, a adição de novas funções de aproximação geram novos graus de liberdade em deslocamento. Nesse trabalho, essas funções serão do tipo "bolha", ou seja, elas devem ter valores nulos nos nós. Isso é conveniente, pois mantém o significado físico dos graus de liberdade originais. 
A base de aproximação original dos deslocamentos no contorno do elemento é formada pelas funções de forma $\phi_{1}$ e $\phi_{2}$, que consistem em uma PU. Essa base é representada por:

$$
\beta_{0}=\left\{\phi_{1}, \phi_{2}\right\}
$$

Com o enriquecimento, novas bases de aproximação são adicionadas. A nova base enriquecida pode ser representada genericamente por:

$$
\beta_{g}=\left\{\phi_{1}, \phi_{2}\right\} \cup \beta_{E}
$$

Sendo $\beta_{E}$ a base enriquecedora formada por funções $h_{j}, j=1, \ldots, n$, resultado da multiplicação de uma PU com uma função de interesse. Portanto, podemos representar $\beta_{g}$ por:

$$
\beta_{g}=\left\{\phi_{1}, \phi_{2}, h_{1}, \ldots, h_{n}\right\}
$$

As três bases enriquecedoras comentadas na seção anterior, e referidas aqui como $\beta_{1}$, $\beta_{2}$ e $\beta_{3}$, serão apresentadas na sequência.

\subsubsection{1- Enriquecimento polinomial com refinamento hierárquico}

Essa base enriquecedora, denominada de $\beta_{1}$, é gerada pela sequência:

$$
\beta_{1}=\left\{\phi_{1}, \phi_{2}\right\} \cup\left\{\xi^{n-1}\left(1-\xi^{2}\right)\right\}, \text { com } n \geq 1 \text { e } n \in \mathbb{Z}
$$

Sendo $\mathbb{Z}$ o conjunto dos números inteiros.

Para ilustrar, os primeiros oito termos da base $\beta_{1}$ valem:

$$
\beta_{1}=\left\{\frac{(\xi+1)}{2}, \frac{(-\xi+1)}{2}, 1-\xi^{2}, \xi\left(1-\xi^{2}\right), \xi^{2}\left(1-\xi^{2}\right), \xi^{3}\left(1-\xi^{2}\right), \xi^{4}\left(1-\xi^{2}\right), \xi^{5}\left(1-\xi^{2}\right)\right\}
$$


Sendo cada termo dessa base igual a $m_{i}$, com $i=1, \ldots, 8$, a representação de cada função é dada pela Figura 3.3:

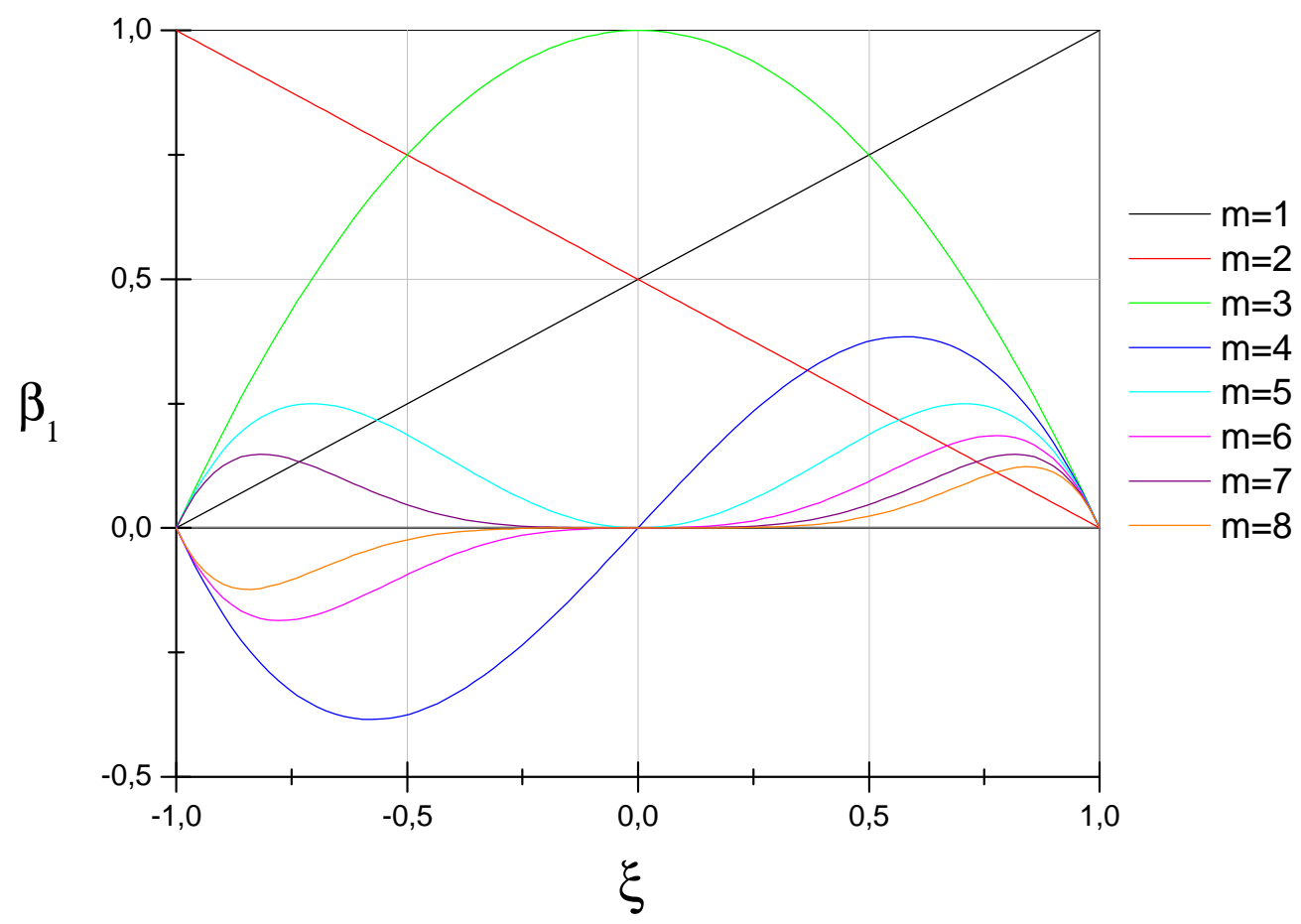

Figura 3.3 - Representação gráfica das funções da base hierárquica

\subsubsection{2- Enriquecimento polinomial com refinamento não hierárquico}

Essa base enriquecedora, denominada de $\beta_{2}$, é gerada pela sequência:

$$
\beta_{2}=\left\{\phi_{1}, \phi_{2}\right\} \cup\left\{(\xi+1)^{2 n}(\xi-1)^{2 n},(\xi+1)^{2 n-1}(\xi-1)^{2 n},-(\xi+1)^{2 n}(\xi-1)^{2 n-1}\right\}, \quad \text { com }
$$

$n \geq 1$ e $n \in \mathbb{Z}$

Sendo $\mathbb{Z}$ o conjunto dos números inteiros.

Para ilustrar, os primeiros oito termos da base $\beta_{2}$ valem:

$$
\begin{aligned}
\beta_{2}= & \left\{\frac{(\xi+1)}{2}, \frac{(-\xi+1)}{2},(\xi+1)^{2}(\xi-1)^{2},(\xi+1)^{1}(\xi-1)^{2},-(\xi+1)^{2}(\xi-1)^{1},(\xi+1)^{4}(\xi-1)^{4},\right. \\
& \left.(\xi+1)^{3}(\xi-1)^{4},-(\xi+1)^{4}(\xi-1)^{3}\right\}
\end{aligned}
$$


Sendo cada termo dessa base igual a $m_{i}, \operatorname{com} i=1, \ldots, 8$, a representação de cada função é dada pela Figura 3.4:

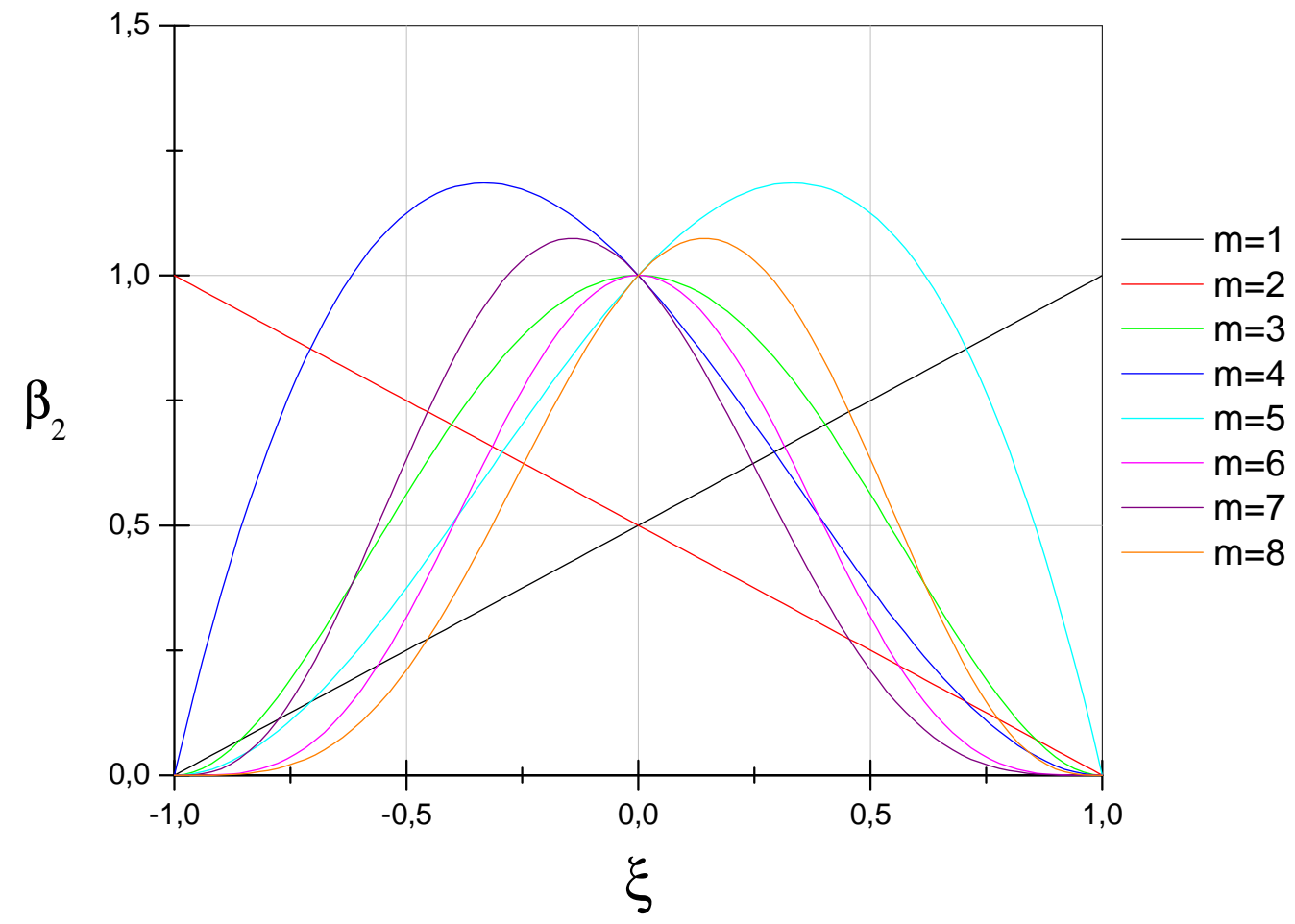

Figura 3.4 - Representação gráfica das funções da base não-hierárquica

\subsubsection{3- Enriquecimento trigonométrico}

Essa base enriquecedora, denominada de $\beta_{3}$, é gerada pela sequência:

$$
\beta_{3}=\left\{\phi_{1}, \phi_{2}\right\} \cup\left\{\left(1-\xi^{2}\right) \cos (n \xi),\left(1-\xi^{2}\right) \operatorname{sen}(n \xi)\right\}, \text { com } n \geq 1 \text { e } n \in \mathbb{Z}
$$

Sendo $\mathbb{Z}$ o conjunto dos números inteiros.

Para ilustrar, os primeiros oito termos da base $\beta_{3}$ valem:

$$
\begin{aligned}
\beta_{3}= & \left\{\frac{(\xi+1)}{2}, \frac{(-\xi+1)}{2},\left(1-\xi^{2}\right) \cos (\xi),\left(1-\xi^{2}\right) \operatorname{sen}(\xi),\left(1-\xi^{2}\right) \cos (2 \xi),\left(1-\xi^{2}\right) \operatorname{sen}(2 \xi),\right. \\
& \left.\left(1-\xi^{2}\right) \cos (3 \xi),\left(1-\xi^{2}\right) \operatorname{sen}(3 \xi)\right\}
\end{aligned}
$$


Sendo cada termo dessa base igual a $m_{i}$, com $i=1, \ldots, 8$, a representação de cada função é dada pela Figura 3.5:

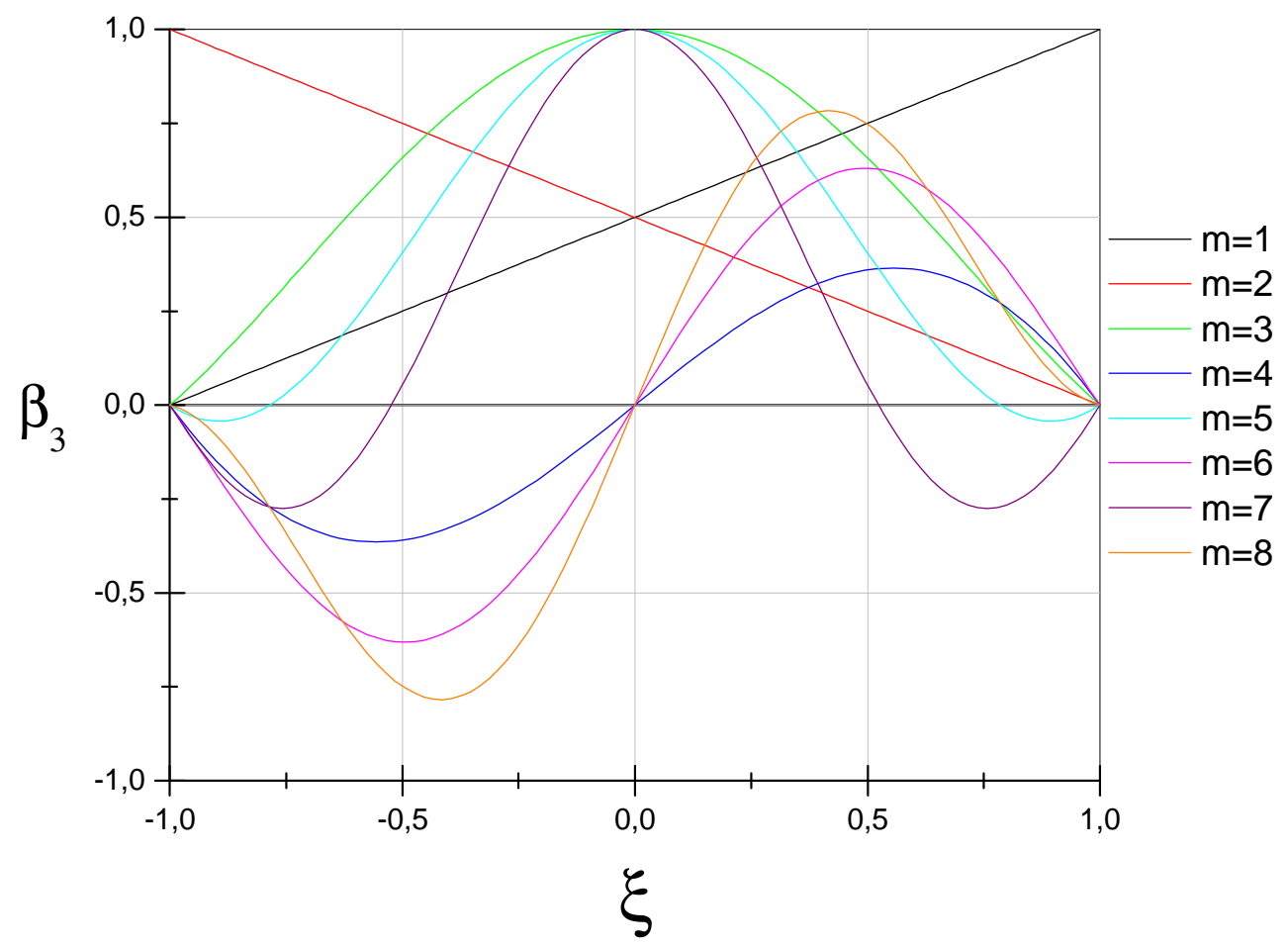

Figura 3.5 - Representação gráfica das funções da base trigonométrica

\subsection{3 - Aproximação do campo de deslocamento numa fronteira enriquecida}

Considere a aproximação de deslocamentos em uma fronteira não enriquecida dada pela Equação (3.31). Como já comentado, o enriquecimento num lado do elemento adiciona novas bases de aproximação à inicial. Portanto, considere um único trecho enriquecido identificado por $\Gamma_{\mathrm{E}}$. A nova aproximação do deslocamento nessa fronteira é dada por:

$$
\tilde{\boldsymbol{u}}_{E}=\widetilde{\boldsymbol{U}}_{E} \boldsymbol{d}_{E}
$$

Sendo $\tilde{\boldsymbol{u}}_{\boldsymbol{E}}$ a aproximação dos deslocamentos após o enriquecimento, $\widetilde{\boldsymbol{U}}_{\boldsymbol{E}}$ a matriz que guarda as bases da aproximação, tanto as originais quanto aquelas devido ao enriquecimento, e $\boldsymbol{d}_{\boldsymbol{E}} \mathrm{o}$ vetor dos parâmetros nodais, constituído tanto pelos originais quanto aqueles devido ao enriquecimento. 
A Equação (3.55) pode também ser representada em notação matricial:

$$
\left\{\begin{array}{l}
u_{x} \\
u_{y}
\end{array}\right\}=\left[\begin{array}{ccccccccc}
\phi_{1} & 0 & \phi_{2} & 0 & h_{1} & 0 & \cdots & h_{n} & 0 \\
0 & \phi_{1} & 0 & \phi_{2} & 0 & h_{1} & \cdots & 0 & h_{n}
\end{array}\right]\left\{\begin{array}{c}
u_{x 1} \\
u_{y 1} \\
u_{x 2} \\
u_{y 2} \\
\Delta u_{x 1} \\
\Delta u_{y 1} \\
\vdots \\
\Delta u_{x n} \\
\Delta u_{y n}
\end{array}\right\}
$$

Sendo $h_{i}$ as funções enriquecedoras, $n$ o número máximo de funções enriquecedoras, $\Delta u_{x i} \mathrm{e}$ $\Delta u_{y i}$ os novos parâmetros provenientes do enriquecimento.

Portanto, pode-se representar a matriz $\widetilde{\boldsymbol{U}}_{\boldsymbol{E}}$ e $\boldsymbol{d}_{\boldsymbol{E}}$ por:

$$
\begin{gathered}
\widetilde{\boldsymbol{U}}_{\boldsymbol{E}}=\left[\begin{array}{ll}
\widetilde{\boldsymbol{U}}_{\Gamma_{E}} & \Delta \widetilde{\boldsymbol{U}}_{\Gamma_{E}}
\end{array}\right] \\
\boldsymbol{d}_{\boldsymbol{E}}=\left[\begin{array}{c}
\boldsymbol{d}_{\Gamma_{E}} \\
\Delta \boldsymbol{d}_{\Gamma_{E}}
\end{array}\right]
\end{gathered}
$$

Onde $\widetilde{\boldsymbol{U}}_{\Gamma_{\mathrm{E}}}$ é a matriz que representa as funções de forma iniciais, $\Delta \widetilde{\boldsymbol{U}}_{\Gamma_{E}}$ representa as bases de aproximação provenientes do enriquecimento, $\boldsymbol{d}_{\Gamma_{E}}$ os graus de liberdade iniciais e $\Delta \boldsymbol{d}_{\Gamma_{E}}$ os novos parâmetros.

\subsection{4- Equacionamento levando em conta o enriquecimento}

Logicamente, ao modificar a aproximação de deslocamento no contorno $\Gamma_{\mathrm{E}}$, as matrizes e vetores envolvidos na formulação também sofrerão modificação.

Assim, a matriz $\boldsymbol{G}$ passa a ser representada por $\boldsymbol{G}_{\boldsymbol{E}}$, indicada na equação abaixo: 


$$
G_{E}=\int_{\Gamma_{E}} T^{T} \widetilde{U}_{E} \mathrm{~d} \Gamma
$$

Utilizando a mesma notação que na seção anterior, a matriz $\boldsymbol{G}_{\boldsymbol{E}}$ pode ser representada por:

$$
\boldsymbol{G}_{\boldsymbol{E}}=\left[\begin{array}{ll}
\boldsymbol{G}_{\Gamma_{E}} & \Delta \boldsymbol{G}_{\Gamma_{E}}
\end{array}\right]
$$

onde a matriz $\boldsymbol{G}_{\Gamma_{E}}$ representa a parcela da aproximação inicial e $\Delta \boldsymbol{G}_{\Gamma_{E}}$ a parcela referente ao enriquecimento. Elas são dadas por:

$$
\begin{gathered}
\boldsymbol{G}_{\Gamma_{E}}=\int_{\Gamma_{E}} \boldsymbol{T}^{\boldsymbol{T}} \widetilde{\boldsymbol{U}}_{\Gamma_{E}} \mathrm{~d} \Gamma \\
\Delta \boldsymbol{G}_{\Gamma_{E}}=\int_{\Gamma_{E}} \boldsymbol{T}^{\boldsymbol{T}} \Delta \widetilde{\boldsymbol{U}}_{\Gamma_{E}} \mathrm{~d} \Gamma
\end{gathered}
$$

Além dessa matriz, o vetor $\boldsymbol{q}$ também sofre uma modificação, passando a ser denotado por $\boldsymbol{q}_{\boldsymbol{E}}$ :

$$
\boldsymbol{q}_{E}=\int_{\Gamma_{E}} \widetilde{\boldsymbol{U}}_{\boldsymbol{E}}^{\boldsymbol{T}} \overline{\boldsymbol{t}} \mathrm{d} \Gamma
$$

ou:

$$
\boldsymbol{q}_{\boldsymbol{E}}=\left[\begin{array}{ll}
\boldsymbol{q}_{\Gamma_{E}} & \Delta \boldsymbol{q}_{\Gamma_{E}}
\end{array}\right]
$$

onde $\boldsymbol{q}_{\Gamma_{E}}$ é a parcela referente à aproximação inicial e $\Delta \boldsymbol{q}_{\Gamma_{E}}$ a referente ao enriquecimento. Elas valem:

$$
\begin{gathered}
\boldsymbol{q}_{\Gamma_{E}}=\int_{\Gamma_{E}} \widetilde{\boldsymbol{U}}_{\Gamma_{E}}^{\boldsymbol{T}} \overline{\mathrm{d}} \mathrm{d} \Gamma \\
\Delta \boldsymbol{q}_{\Gamma_{E}}=\int_{\Gamma_{E}} \Delta \widetilde{\boldsymbol{U}}_{\Gamma_{E}}^{\boldsymbol{T}} \overline{\boldsymbol{t}} \mathrm{d} \Gamma
\end{gathered}
$$


Com as novas matrizes e vetores definidos, parte-se para a montagem do sistema do elemento.

Tendo em mãos as equações (3.58), (3.60) e (3.64), pode-se ver que o sistema do elemento finito sofre um acréscimo de termos na condição de compatibilidade e um acréscimo de relações de reciprocidade devido ao enriquecimento. Ele pode ser representado por:

$$
\left[\begin{array}{ccc}
\boldsymbol{F} & -\boldsymbol{G} & -\Delta \boldsymbol{G}_{\Gamma_{E}} \\
-\boldsymbol{G}^{\boldsymbol{T}} & 0 & 0 \\
-\Delta \boldsymbol{G}_{\Gamma_{E}}^{\boldsymbol{T}} & 0 & 0
\end{array}\right]\left\{\begin{array}{c}
\boldsymbol{c} \\
\boldsymbol{d} \\
\Delta \boldsymbol{d}_{\Gamma_{E}}
\end{array}\right\}=\left\{\begin{array}{c}
\boldsymbol{e} \\
-\boldsymbol{q} \\
-\Delta \boldsymbol{q}_{\Gamma_{E}}
\end{array}\right\}
$$

O sistema acima foi montado considerando apenas um trecho enriquecido de um elemento. Percebe-se que mais de um trecho do elemento pode ser enriquecido. Portanto, para escrever em uma forma geral, o sistema linear para um elemento é dado por:

$$
\left[\begin{array}{ccc}
\boldsymbol{F} & -\boldsymbol{G} & -\Delta \boldsymbol{G} \\
-\boldsymbol{G}^{\boldsymbol{T}} & 0 & 0 \\
-\Delta \boldsymbol{G}^{\boldsymbol{T}} & 0 & 0
\end{array}\right]\left\{\begin{array}{c}
\boldsymbol{c} \\
\boldsymbol{d} \\
\Delta \boldsymbol{d}
\end{array}\right\}=\left\{\begin{array}{c}
\boldsymbol{e} \\
-\boldsymbol{q} \\
-\Delta \boldsymbol{q}
\end{array}\right\}
$$

onde $\Delta \boldsymbol{d}$ é o vetor que representa os parâmetros nodais totais vindos do enriquecimento, $\Delta \boldsymbol{G}$ a matriz referente à parcela do enriquecimento e $\Delta \boldsymbol{q}$ o vetor que promove a contribuição das cargas externas devido ao enriquecimento.

Vale destacar que a existência e a unicidade da solução continuam atreladas à condição (3.45), sendo que os parâmetros do vetor $\Delta \boldsymbol{d}$ devem ser levados em conta nessa equação.

\subsection{5- Montagem do sistema global considerando o enriquecimento}

O sistema dado por (3.68) é válido apenas para um elemento com um ou mais trechos enriquecidos. Para a construção do sistema de uma rede de elementos finitos, os novos parâmetros vindos do enriquecimento numa fronteira $\Gamma_{\boldsymbol{E}}$ comum a dois elementos vizinhos devem atender às mesmas condições dos graus de liberdade original. 
Assim, dados dois elementos $i$ e $j$ com um ou mais trechos em comum, os deslocamentos devem atender a uma condição de continuidade em forma forte, o que implica em impor mesmos enriquecimentos nos trechos dos elementos que irão compor a fronteira comum. Essa condição se exprime por:

$$
\tilde{\boldsymbol{u}}_{E}^{i}=\tilde{\boldsymbol{u}}_{\mathrm{E}}^{j}
$$

Já a condição adicional de reciprocidade das tensões é imposta em forma fraca mediante a utilização da matriz das bases de aproximação devido ao enriquecimento como função ponderadora. Considerando um caso geral em que existam forças de superfície $\overline{\boldsymbol{t}}$ na fronteira enriquecida, tem-se:

$$
\int_{\Gamma_{E}} \Delta \widetilde{U}_{\Gamma_{E}}^{\boldsymbol{T}}\left(\boldsymbol{t}^{i}+\boldsymbol{t}^{j}-\overline{\boldsymbol{t}}\right) \mathrm{d} \Gamma=0
$$

Os graus de liberdade originais ainda atendem ao equilíbrio dado por (3.50)

O sistema global para uma rede contendo $n$ elementos finitos com um total de $n_{t}$ graus de liberdade em deslocamentos e $\Delta n_{t}$ graus de liberdade provenientes do enriquecimento é representado por:

$$
\left(\begin{array}{cccccc}
F_{1} & 0 & \cdots & 0 & \overbrace{-G_{1}}^{1, \ldots, n_{t}} & \overbrace{-\Delta G_{1}}^{1, \ldots, n_{t}} \\
0 & F_{2} & \cdots & 0 & -G_{2} & -\Delta G_{2} \\
\vdots & \vdots & \ddots & \vdots & \vdots & \vdots \\
0 & 0 & \cdots & F_{n} & -G_{n} & -\Delta G_{n} \\
-G_{1}^{T} & -G_{2}^{T} & \cdots & -G_{n}^{T} & 0 & 0 \\
-\Delta G_{1}^{T} & -\Delta G_{2}^{T} & \cdots & -\Delta G_{n}^{T} & 0 & 0
\end{array}\right)\left\{\begin{array}{c}
c_{1} \\
c_{2} \\
\vdots \\
c_{n} \\
d_{t} \\
\Delta d_{t}
\end{array}\right\}=\left\{\begin{array}{c}
e_{1} \\
e_{2} \\
\vdots \\
e_{n} \\
-q_{t} \\
-\Delta q_{t}
\end{array}\right\}
$$

onde $\Delta \boldsymbol{d}_{t}$ é o vetor que coleta os $\Delta n_{t}$ parâmetros em deslocamento e $\Delta \boldsymbol{q}_{t}$ o vetor que representa as forças na fronteira enriquecida.

As matrizes $\Delta \boldsymbol{G}_{\boldsymbol{i}}$ e $\Delta \boldsymbol{G}_{\boldsymbol{j}}$ referentes a fronteira enriquecida $\Gamma_{\mathrm{E}}$, devem ser inseridas no sistema na mesma coluna, porém nas linhas correspondentes à cada elemento. 
Um esboço mais detalhado da montagem do sistema global considerando o enriquecimento pode ser encontrado no trabalho de SOUZA (2008).

Mais uma vez, a condição de existência e unicidade da solução deve atender ao teste do "mosaico". Lembrando que os parâmetros acrescidos devido ao enriquecimento devem ser considerados. 


\section{4- 0 Método dos Elementos Finitos Generalizados}

\section{1- Considerações iniciais}

Além das formulações descritas no capítulo anterior, outros métodos surgiram a fim de superar algumas limitações do MEF, destacando-se, entre eles, os denominados métodos sem malhas. Tais métodos se caracterizam por gerar uma aproximação para o problema utilizando uma distribuição dispersa de nós no domínio do sólido, dispensando o uso de redes de elementos finitos. Cada nó possui um domínio de influência, denominado nuvem sobre o qual se define uma aproximação local. As funções de aproximação podem variar para cada nuvem.

Uma vantagem dessa técnica é a facilidade de utilizar funções especiais para uma melhor aproximação da solução do problema, ao contrário do MEF, onde, em geral, as funções são polinomiais. Essas funções especiais permitem uma melhor aproximação da solução em problemas que contenham singularidades. Um limitante desses métodos é a compatibilização das aproximações locais para construir a aproximação global. Outro limitante é que o aumento do grau da aproximação global exige a adição de nós no domínio do sólido, aumentando o custo computacional da análise.

A fim de proporcionar conformidade para a aproximação global, o conceito de funções PU foi introduzido.

A função PU em um dado nó $j$, representada por $\phi_{j}(x)$, possui as seguintes características:

- $\quad \phi_{j}(x) \geq 0$ em todo o domínio, sendo $j$ o número do nó;

- $\quad \sum_{j=1}^{n} \phi_{j}(x)=1$, onde $n$ é o número de nós;

- $\phi_{j}(x)$ deve ser igual a um no nó $j$ e decrescer até o valor nulo nos demais nós.

A fim de evitar a adição de novos nós no domínio para a construção de funções de aproximação de grau elevado, Duarte e Oden (1996) propuseram o Método das Nuvens hp. 
Tal método consiste em construir uma aproximação inicial baseada em uma PU polinomial de baixa ordem e enriquecê-la mediante sua multiplicação por outras funções de interesse com um correspondente acréscimo de novos parâmetros nodais. A denominação " $h p$ " do método é devido à facilidade de implementar o refinamento $h$ (aumento de pontos nodais) e o refinamento $p$ (adição de novas funções na aproximação).

Por outro lado, Melenk e Babuška (1996) propuseram empregar uma rede de elementos finitos de modo a aproveitar as funções de forma utilizadas no MEF para compor a PU. Assim, surgiu o Método dos Elementos Finitos Partição da Unidade (MEFPU).

A combinação do MEFPU com o Método das Nuvens $h p$ levou a criação do MEFG (DUARTE; ODEN; BABUŠKA, 2000). Essa união trouxe as seguintes vantagens:

- Facilidade na geração da PU (vantagem vinda do MEF);

- A nuvem atrelada a determinado nó é definida pelo conjunto de elementos finitos que tenham esse nó em comum (Figura 4.1) (vantagem vinda do MEF);

- Facilidade em impor as condições de contorno essenciais (vantagem vinda do MEF);

- Facilidade em utilizar funções de aproximação especiais no enriquecimento em forma seletiva sobre regiões do domínio (vantagem vinda do Método das Nuvens $h p$ );

- O MEFG não é sensível à distorção da rede, já que o enriquecimento é feito em coordenadas globais.

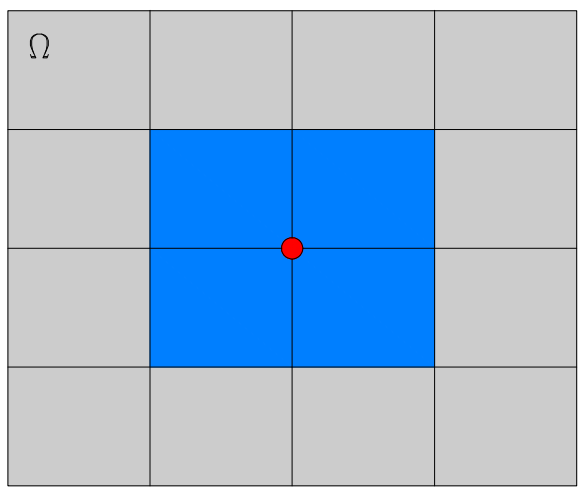

Nó enriquecido

$\square$ Nuvem

Figura 4.1 - Nuvem enriquecedora 
Tendo-se em vista suas características gerais, o MEFG propõe a construção de aproximações locais atreladas a nós (centro de nuvens formadas pelos elementos que os dividem). Em cada nuvem as funções de forma básicas de cada elemento são tomadas como PU. O método promove o enriquecimento da aproximação nodal nas nuvens definidas sobre regiões de interesse. Esse enriquecimento é dado pela multiplicação de uma PU por uma função enriquecedora $f$, polinomial ou não. Pode-se também utilizar mais de uma função enriquecedora no mesmo nó. Portanto, a família de funções de forma do MEFG pode ser representada por:

$$
\mathbb{F}_{n}=\left\{\Phi_{\alpha}^{i}, \alpha=1, \ldots, n / i=1, \ldots, n f\right\}
$$

sendo:

$\alpha$ - o índice que representa a nuvem;

$n$ - o número total de nuvens;

$i$ - o contador para o número de funções enriquecedoras;

nf - o número total de funções enriquecedoras;

$\Phi$ - a função resultante do produto entre a PU e a função enriquecedora $f_{i}$ da nuvem

Nota-se que o enriquecimento por uma função polinomial de uma PU também polinomial pode gerar dependências lineares que se refletem num sistema resolutivo com matriz dos coeficientes positiva semi-definida. Não obstante tal limitação, o sistema pode ser resolvido eficientemente utilizando um procedimento iterativo proposto por Strouboulis, Babuška e Copps (2000).

Mais informações sobre as potencialidades e aplicações do MEFG podem ser encontradas nos trabalhos de Barros (2002), Torres (2003), Proença e Torres (2008), Duarte e Kim (2008), Proença (2008), Alves, Argôlo, e Proença, (2009). 


\section{2- Formulação do elemento finito}

Dada a característica de definição das nuvens no MEFG, as funções de forma do método podem ser construídas observando-se os elementos finitos diretamente. Nesse sentido toda a estrutura original do MEF pode ser preservada.

O elemento finito utilizado neste trabalho é o elemento quadrilateral, bilinear, com dois graus de liberdade por nó (deslocamentos na direção x e y) e isoparamétrico (ASSAN, 2003). Em seguida, expõem-se as equações para a montagem do elemento sem enriquecimento e com enriquecimento.

\subsection{1- Elemento sem enriquecimento}

Considere o elemento finito em coordenadas cartesianas globais representado na Figura 4.2 .

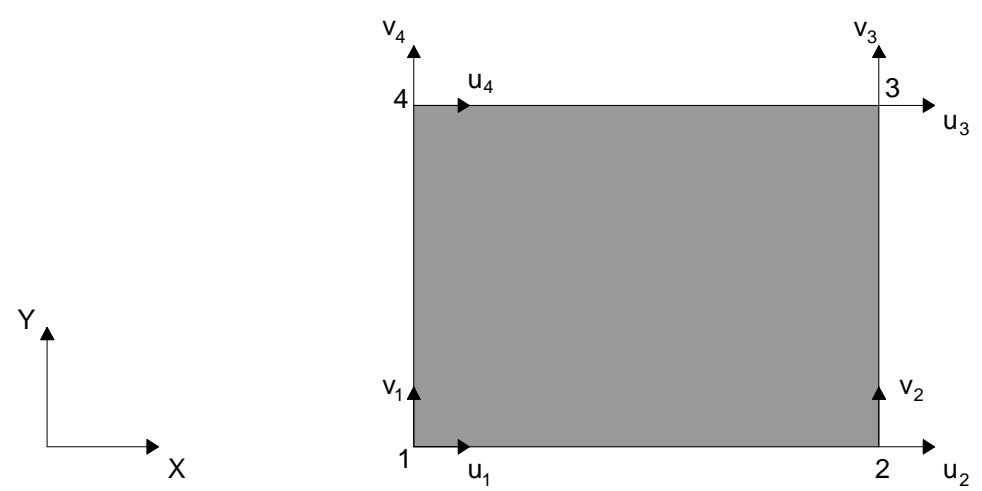

Figura 4.2 - Elemento finito em coordenadas cartesianas

Para facilitar o equacionamento, adota-se um elemento de referência definido num sistema de

coordenadas adimensionais $(\xi, \eta)$, onde $\xi=\frac{x}{a}$ e $\eta=\frac{y}{a}$, com origem em seu baricentro (Figura 4.3). 


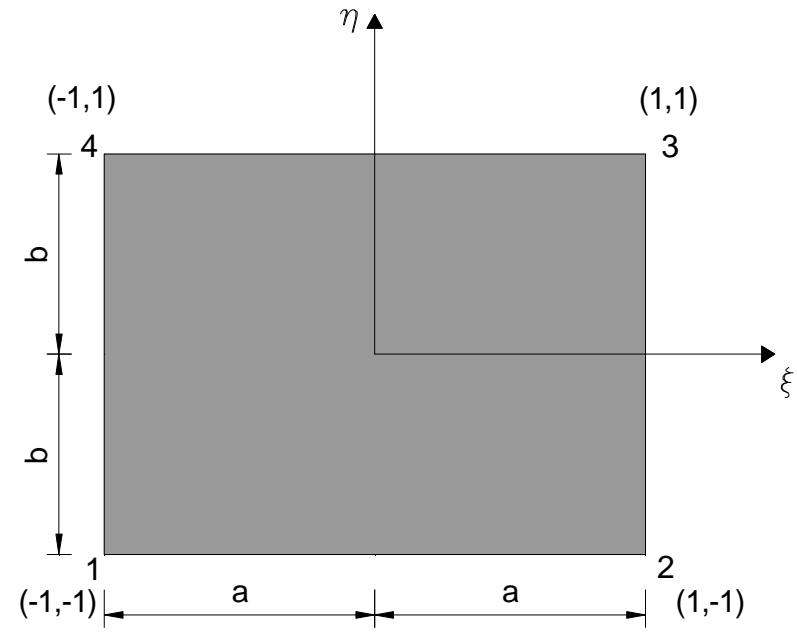

Figura 4.3 - Elemento finito em coordenadas adimensionais

As funções de forma do elemento atreladas a cada nó valem:

$$
\begin{aligned}
\phi_{1} & =\frac{1}{4}(1-\xi)(1-\eta) \\
\phi_{2} & =\frac{1}{4}(1+\xi)(1-\eta) \\
\phi_{3} & =\frac{1}{4}(1+\xi)(1+\eta) \\
\phi_{4} & =\frac{1}{4}(1-\xi)(1+\eta)
\end{aligned}
$$

Assim, de acordo com a formulação isoparamétrica, a aproximação dos deslocamentos num ponto qualquer do elemento pode ser representada matricialmente por:

$$
\left\{\begin{array}{l}
u \\
v
\end{array}\right\}=\underbrace{\left[\begin{array}{cccccccc}
\phi_{1} & 0 & \phi_{2} & 0 & \phi_{3} & 0 & \phi_{4} & 0 \\
0 & \phi_{1} & 0 & \phi_{2} & 0 & \phi_{3} & 0 & \phi_{4}
\end{array}\right]}_{\phi}\left\{\begin{array}{l}
u_{1} \\
v_{1} \\
u_{2} \\
v_{2} \\
u_{3} \\
v_{3} \\
u_{4} \\
v_{4}
\end{array}\right\}
$$

onde $u$ e $v$ são os deslocamentos nas direções $\mathrm{x}$ e $\mathrm{y}$, respectivamente e $u_{i}$ e $v_{i}$ os deslocamentos ou graus de liberdade do nó $i$ nas direções x e y, respectivamente. 
A equação de compatibilidade é dada por:

$$
\left\{\begin{array}{c}
\varepsilon_{x} \\
\varepsilon_{y} \\
\gamma_{x y}
\end{array}\right\}=\left[\begin{array}{cc}
\frac{\partial}{\partial x} & 0 \\
0 & \frac{\partial}{\partial y} \\
\frac{\partial}{\partial y} & \frac{\partial}{\partial x}
\end{array}\right]\left\{\begin{array}{l}
u \\
v
\end{array}\right\}
$$

Substituindo a Equação (4.3) em (4.4), obtém-se:

$$
\left\{\begin{array}{c}
\varepsilon_{x} \\
\varepsilon_{y} \\
\gamma_{x y}
\end{array}\right\}=\underbrace{\left[\begin{array}{cccccccc}
\frac{\partial \phi_{1}}{\partial x} & 0 & \frac{\partial \phi_{2}}{\partial x} & 0 & \frac{\partial \phi_{3}}{\partial x} & 0 & \frac{\partial \phi_{4}}{\partial x} & 0 \\
0 & \frac{\partial \phi_{1}}{\partial y} & 0 & \frac{\partial \phi_{2}}{\partial y} & 0 & \frac{\partial \phi_{3}}{\partial y} & 0 & \frac{\partial \phi_{4}}{\partial y} \\
\frac{\partial \phi_{1}}{\partial y} & \frac{\partial \phi_{1}}{\partial x} & \frac{\partial \phi_{2}}{\partial y} & \frac{\partial \phi_{2}}{\partial x} & \frac{\partial \phi_{3}}{\partial y} & \frac{\partial \phi_{3}}{\partial x} & \frac{\partial \phi_{4}}{\partial y} & \frac{\partial \phi_{4}}{\partial x}
\end{array}\right]}_{B}\left\{\begin{array}{c}
u_{1} \\
v_{1} \\
u_{2} \\
v_{2} \\
u_{3} \\
v_{3} \\
u_{4} \\
v_{4}
\end{array}\right\}
$$

Nota-se que o vetor de deformações está sendo representado em coordenadas cartesianas. Para que sejam utilizadas coordenadas adimensionais $(\xi, \eta)$, é necessário recorrer à regra da cadeia dada por:

$$
\begin{aligned}
& \varepsilon_{x}=\frac{\partial u}{\partial x}=\frac{\partial u}{\partial \xi} \frac{\partial \xi}{\partial x}+\frac{\partial u}{\partial \eta} \frac{\partial \eta}{\partial x} \\
& \varepsilon_{y}=\frac{\partial v}{\partial y}=\frac{\partial v}{\partial \xi} \frac{\partial \xi}{\partial y}+\frac{\partial v}{\partial \eta} \frac{\partial \eta}{\partial y} \\
& \gamma_{x y}=\frac{\partial u}{\partial y}+\frac{\partial v}{\partial x}=\frac{\partial u}{\partial \xi} \frac{\partial \xi}{\partial y}+\frac{\partial u}{\partial \eta} \frac{\partial \eta}{\partial y}+\frac{\partial v}{\partial \xi} \frac{\partial \xi}{\partial x}+\frac{\partial v}{\partial \eta} \frac{\partial \eta}{\partial x}
\end{aligned}
$$

A fim de modelar adequadamente os contornos irregulares do problema, foi utilizado o elemento finito isoparamétrico. As coordenadas de um ponto qualquer desse elemento são mapeadas utilizando as funções de forma descritas em (4.2): 


$$
\left\{\begin{array}{l}
x \\
y
\end{array}\right\}=\underbrace{\left[\begin{array}{cccccccc}
\phi_{1} & 0 & \phi_{2} & 0 & \phi_{3} & 0 & \phi_{4} & 0 \\
0 & \phi_{1} & 0 & \phi_{2} & 0 & \phi_{3} & 0 & \phi_{4}
\end{array}\right]}_{\phi}\left\{\begin{array}{l}
x_{1} \\
y_{1} \\
x_{2} \\
y_{2} \\
x_{3} \\
y_{3} \\
x_{4} \\
y_{4}
\end{array}\right\}
$$

onde $x$ e $y$ são as coordenadas de um ponto genérico do elemento e $x_{i}$ e $y_{i}$ as coordenadas do nó $i$ do elemento.

As regras da cadeia dadas em (4.6) podem ser representadas por:

$$
\left\{\begin{array}{l}
\frac{\partial u}{\partial \xi} \\
\frac{\partial u}{\partial \eta} \\
\frac{\partial v}{\partial \xi} \\
\frac{\partial v}{\partial \eta}
\end{array}\right\}=\left[\begin{array}{cccc}
\frac{\partial x}{\partial \xi} & \frac{\partial y}{\partial \xi} & 0 & 0 \\
\frac{\partial x}{\partial \eta} & \frac{\partial y}{\partial \eta} & 0 & 0 \\
0 & 0 & \frac{\partial x}{\partial \xi} & \frac{\partial y}{\partial \xi} \\
0 & 0 & \frac{\partial x}{\partial \eta} & \frac{\partial y}{\partial \eta}
\end{array}\right]\left\{\begin{array}{l}
\frac{\partial u}{\partial x} \\
\frac{\partial u}{\partial y} \\
\frac{\partial v}{\partial y}
\end{array}\right\}
$$

Assim, a matriz Jacobiana, responsável pela transformação das coordenadas locais pelas coordenadas globais, é definida por:

$$
J=\left[\begin{array}{ll}
\frac{\partial x}{\partial \xi} & \frac{\partial y}{\partial \xi} \\
\frac{\partial x}{\partial \eta} & \frac{\partial y}{\partial \eta}
\end{array}\right]
$$

a Equação (4.8) passa a ser escrita por: 


$$
\left\{\begin{array}{l}
\frac{\partial u}{\partial \xi} \\
\frac{\partial u}{\partial \eta} \\
\frac{\partial v}{\partial \xi} \\
\frac{\partial v}{\partial \eta}
\end{array}\right\}=\left[\begin{array}{ll}
J & 0 \\
0 & J
\end{array}\right]\left\{\begin{array}{l}
\frac{\partial u}{\partial y} \\
\frac{\partial v}{\partial x} \\
\frac{\partial v}{\partial y}
\end{array}\right\}
$$

Agora, utilizando as igualdades dadas em (4.6) e explicitando as derivadas de $u$ e $v$ em relação $x$ e a $y$, as equações podem ser representadas por:

$$
\left\{\begin{array}{l}
\frac{\partial u}{\partial x} \\
\frac{\partial u}{\partial y} \\
\frac{\partial v}{\partial x} \\
\frac{\partial v}{\partial y}
\end{array}\right\}=\left[\begin{array}{cccc}
\frac{\partial \xi}{\partial x} & \frac{\partial \eta}{\partial x} & 0 & 0 \\
\frac{\partial \xi}{\partial y} & \frac{\partial \eta}{\partial y} & 0 & 0 \\
0 & 0 & \frac{\partial \xi}{\partial x} & \frac{\partial \eta}{\partial x} \\
0 & 0 & \frac{\partial \xi}{\partial y} & \frac{\partial \eta}{\partial y}
\end{array}\right]\left\{\begin{array}{l}
\frac{\partial u}{\partial \xi} \\
\frac{\partial u}{\partial \eta} \\
\frac{\partial v}{\partial \xi} \\
\frac{\partial v}{\partial \eta}
\end{array}\right\}
$$

definindo a matriz inversa da matriz Jacobiana:

$$
H=\left[\begin{array}{ll}
\frac{\partial \xi}{\partial x} & \frac{\partial \eta}{\partial x} \\
\frac{\partial \xi}{\partial y} & \frac{\partial \eta}{\partial y}
\end{array}\right]
$$

a Equação (4.11) pode ser representada por:

$$
\left\{\begin{array}{l}
\frac{\partial u}{\partial x} \\
\frac{\partial u}{\partial y} \\
\frac{\partial v}{\partial x} \\
\frac{\partial v}{\partial y}
\end{array}\right\}=\left[\begin{array}{ll}
H & 0 \\
0 & H
\end{array}\right]\left\{\begin{array}{l}
\frac{\partial u}{\partial \xi} \\
\frac{\partial u}{\partial \eta} \\
\frac{\partial v}{\partial \xi} \\
\frac{\partial v}{\partial \eta}
\end{array}\right\}
$$


$\mathrm{O}$ vetor $(3 \times 1)$ da relação de compatibilidade passa a ser escrito como um vetor $(4 \times 1)$ através da seguinte transformação:

$$
\left\{\begin{array}{l}
\varepsilon_{x} \\
\varepsilon_{y} \\
\gamma_{x y}
\end{array}\right\}=\left[\begin{array}{llll}
1 & 0 & 0 & 0 \\
0 & 0 & 0 & 1 \\
0 & 1 & 1 & 0
\end{array}\right]\left\{\begin{array}{l}
\frac{\partial u}{\partial x} \\
\frac{\partial u}{\partial y} \\
\frac{\partial v}{\partial x} \\
\frac{\partial v}{\partial y}
\end{array}\right\}
$$

da Equação (4.3) tem-se que:

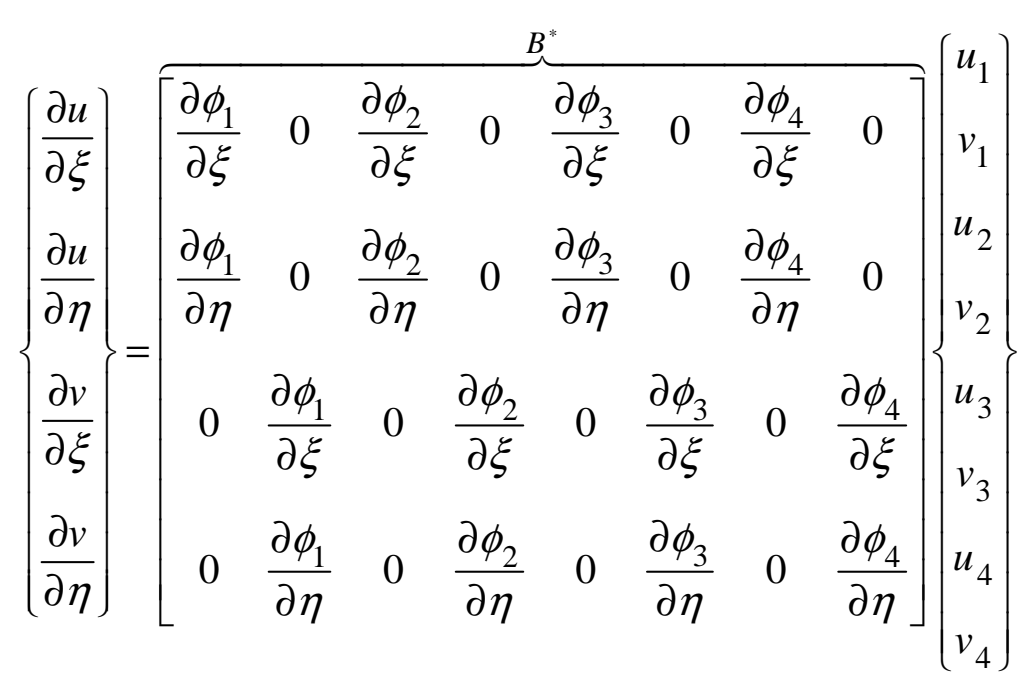

A matriz $B$ relaciona as deformações aos graus de liberdade. Portanto, ela pode ser obtida combinando as equações (4.13), (4.14) e (4.15), resultando:

$$
B=\left[\begin{array}{llll}
1 & 0 & 0 & 0 \\
0 & 0 & 0 & 1 \\
0 & 1 & 1 & 0
\end{array}\right]\left[\begin{array}{ll}
H & 0 \\
0 & H
\end{array}\right]\left[B^{*}\right]
$$

A matriz de rigidez do elemento é representada por:

$$
K_{e}=\int_{-a-b}^{a} \int_{-b}^{b} B^{T} D B d x d y
$$


sendo a matriz $D$ definida em (2.4). Transformando a equação acima em coordenadas adimensionais, tem-se:

$$
K_{e}=\int_{-1}^{1} \int_{-1}^{1} B^{T} D B J d \xi d \eta
$$

onde:

$$
d x d y=J d \xi d \eta
$$

De maneira análoga, o vetor de forças nodais equivalentes é representado por:

$$
r=\int_{-1}^{1} \int_{-1}^{1} \phi^{T} \phi_{q} q J d \xi d \eta
$$

sendo $\phi_{q}$ o vetor das funções de aproximação do carregamento. No caso, $\phi_{q}$ foi utilizado igual a $\phi$.

\subsection{1- Elemento com enriquecimento}

Para ilustrar a formulação devido ao enriquecimento, considere o elemento finito com os quatro nós enriquecidos dado pela Figura 4.4.

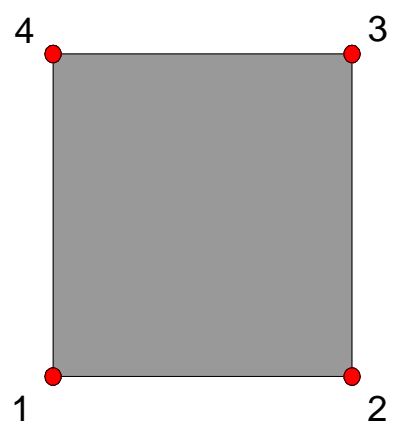

Figura 4.4 - Elemento finito com os quatro nós enriquecidos

A aproximação dos deslocamentos desse elemento é dada por: 


$$
\begin{aligned}
& u=\sum_{i=1}^{4} \phi_{i}\left(u_{i}+\sum_{j=1}^{n f} f_{j}^{u_{i}} b_{j}^{u_{i}}\right) \\
& v=\sum_{i=1}^{4} \phi_{i}\left(v_{i}+\sum_{j=1}^{n f} f_{j}^{v_{i}} b_{j}^{v_{i}}\right)
\end{aligned}
$$

sendo:

$f_{j}^{d_{i}}$ - a função enriquecedora $j$ do nó $i$ referente ao grau de liberdade $d$;

$n f$ - número total de funções enriquecedoras;

$b_{j}^{d_{i}}$ - parâmetro adicional $j$ devido ao enriquecimento do nó $i$ referente ao grau de liberdade $d$.

Um trecho da Equação (4.21) de um nó genérico $i$ pode ser representado de forma matricial por:

$$
\left\{\begin{array}{l}
u \\
v
\end{array}\right\}=\left[\begin{array}{cccccccccccc}
\cdots & \phi_{i} & \phi_{i} f_{1}^{u_{i}} & \cdots & \phi_{i} f_{n f}^{u_{i}} & 0 & 0 & \cdots & 0 & \cdots \\
\cdots & 0 & 0 & \cdots & 0 & \phi_{i} & \phi_{i} f_{1}^{v_{i}} & \cdots & \phi_{i} f_{n f}^{v_{i}} & \cdots
\end{array}\right]\left\{\begin{array}{c}
\vdots \\
u_{i} \\
b_{1}^{u_{i}} \\
\vdots \\
b_{n f}^{u_{i}} \\
v_{i} \\
b_{1}^{v_{i}} \\
\vdots \\
b_{n f}^{v_{i}} \\
\vdots
\end{array}\right\}
$$

e o trecho da matriz $B_{e}^{*}$ ( matriz $B^{*}$ enriquecida) referente a um nó genérico $i$ é dado por: 


$$
\begin{aligned}
& B_{e}^{*}=\left[\begin{array}{ccccc}
\cdots & \frac{\partial \phi_{i}}{\partial \xi} & \frac{\partial \phi_{i}}{\partial \xi} f_{1}^{u_{i}}+\phi_{i} \frac{\partial f_{1}^{u_{i}}}{\partial \xi} & \cdots & \frac{\partial \phi_{i}}{\partial \xi} f_{n f}^{u_{i}}+\phi_{i} \frac{\partial f_{n f}^{u_{i}}}{\partial \xi} \mid \\
\cdots & \frac{\partial \phi_{i}}{\partial \eta} \frac{\partial \phi_{i}}{\partial \eta} f_{1}^{u_{i}}+\phi_{i} \frac{\partial f_{1}^{u_{i}}}{\partial \eta} & \cdots & \frac{\partial \phi_{i}}{\partial \eta} f_{n f}^{u_{i}}+\phi_{i} \frac{\partial f_{n f}^{u_{i}}}{\partial \eta} \mid \\
\cdots & 0 & 0 & \cdots & 0 \\
\cdots & 0 & 0 & \cdots & 0
\end{array}\right.
\end{aligned}
$$

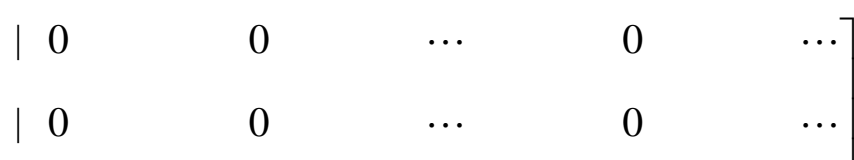

$$
\begin{aligned}
& \mid \frac{\partial \phi_{i}}{\partial \xi} \frac{\partial \phi_{i}}{\partial \xi} f_{1}^{v_{i}}+\phi_{i} \frac{\partial f_{1}^{v_{i}}}{\partial \xi} \cdots \frac{\partial \phi_{i}}{\partial \xi} f_{n f}^{v_{i}}+\phi_{i} \frac{\partial f_{n f}^{v_{i}}}{\partial \xi} \cdots \\
& \left.\mid \frac{\partial \phi_{i}}{\partial \eta} \frac{\partial \phi_{i}}{\partial \eta} f_{1}^{v_{i}}+\phi_{i} \frac{\partial f_{1}^{v_{i}}}{\partial \eta} \cdots \frac{\partial \phi_{i}}{\partial \eta} f_{n f}^{v_{i}}+\phi_{i} \frac{\partial f_{n f}^{v_{i}}}{\partial \eta} \cdots\right]
\end{aligned}
$$

A matriz de rigidez e o vetor de forças nodais equivalentes do elemento são obtidos pelas equações (4.18) e (4.20), respectivamente. Numa rede de elementos finitos, a matriz de rigidez e o vetor de forças nodais globais devem ser formados pela contribuição de cada elemento, lembrando que os parâmetros adicionais devem ser considerados no sistema resolutivo.

Vale destacar que o enriquecimento deve ser realizado sobre as coordenadas globais. 


\section{5- O Método da Partição}

\section{1- Considerações iniciais}

Nesse capítulo descreve-se o Método da Partição ("splitting method") (ANDERSSON; BABUŠKA; STEHLIN, 1998; BABUŠKA; ANDERSSON, 2005). Cada passo do método é apresentado utilizando como exemplo um problema simples da mecânica da fratura. Apesar de ser um problema bidimensional com apenas duas fissuras, a modelagem aqui descrita pode ser estendida para problemas com várias fissuras, estando contidas num meio tridimensional. Trabalhos complementares sobre o método podem ser encontrados em Alves, Argôlo e Proença (2009) e Alves (2010).

O Método da Partição foi apresentado pela primeira vez com o nome de splitting method por Andersson, Babuška e Stehlin (1998) como um procedimento para análise de sólidos com múltiplas fissuras. O referido método tem por base o Princípido da Sobreposição (LACHENBRUCH, 1961; ALIABADI; ROOKE, 1991) e o Princípio de Bueckner (BUECKNER, 1958). O primeiro princípio resolve o problema de uma única fissura pela sobreposição de dois subproblemas: um em que o sólido com as condições de contorno prescritas é analisado sem a presença da fissura e o outro refere-se ao problema da fissura imersa num meio infinito. O Princípio de Bueckner garante que o fator de intensidade de tensão (FIT) de uma fissura inserida em chapa sob certo carregamento externo pode ser determinado de modo equivalente num problema de fissura imersa num meio infinito com carregamento aplicado nas suas faces.

Outros métodos, como o "Alternating Method", aplicado originalmente por Smith, Emery e Kobayashi ${ }^{4}$ (1967 apud ALIABADI; ROOKE, 1991), também utilizam do Princípio da Sobreposição, podendo todos eles serem referenciados como Métodos de Decomposição. O que difere essencialmente o "Splitting Method" do "Alternating Method" é que o primeiro elimina o procedimento iterativo presente no segundo. Isto é feito pela proposição de um terceiro subproblema que compõe a sobreposição.

\footnotetext{
${ }^{4}$ SMITH, F.W., EMERY, A. F., \& KOBAYASHI, A. S. (1967). Stress intensity factor for semi-circular cracks, Part 2 Semi-infinite solid. Jounal of Applied Mechanics, 34, 953-959.
} 
Assim, inicialmente, o Método da Partição procura resolver o problema original dividindo-o em três subproblemas. A combinação entre os três subproblemas é tal a obedecer a condição de nulidade de tensões nas faces da fissura. Para um melhor entendimento do método, considere-se o problema bidimensional ilustrado na Figura 5.1:

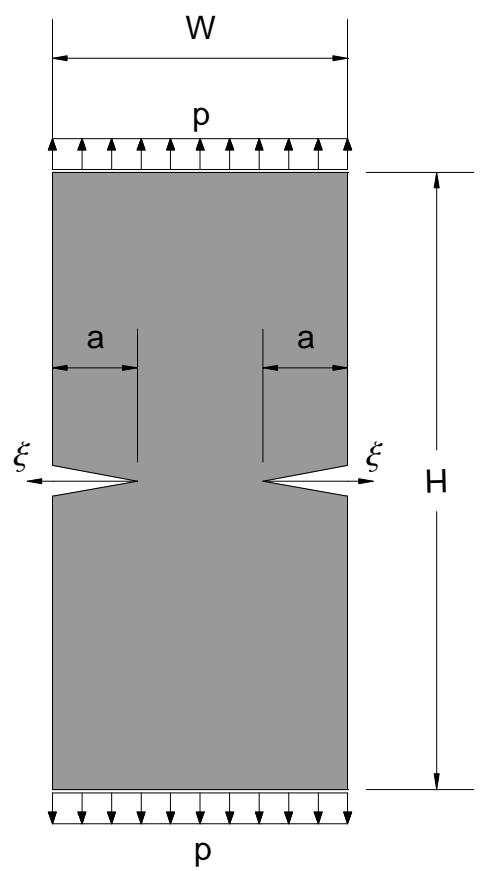

Figura 5.1 - Subproblema original $\boldsymbol{P}_{G}$.

A Figura 5.1 mostra uma chapa de largura $W$ e altura $H$, submetida a um carregamento externo $p$ e contendo duas fissuras com comprimentos iguais a a. O objetivo do problema é obter o FIT para ambas fissuras.

Para auxiliar a solução numérica, adota-se um eixo local de coordenada $\xi$ cuja origem está na ponta da fissura e segue em direção a sua raiz (no caso, a borda da chapa). Cada um dos três subproblemas que compõem o Método da Partição são detalhados em seguida.

\section{2- 10 Subproblema}

O primeiro desses subproblemas é dito subproblema global $\left(P_{G}^{(0)}\right)$ e consiste na análise do problema original com as condições de contorno impostas, porém sem a presença de fissuras (Figura 5.2). 


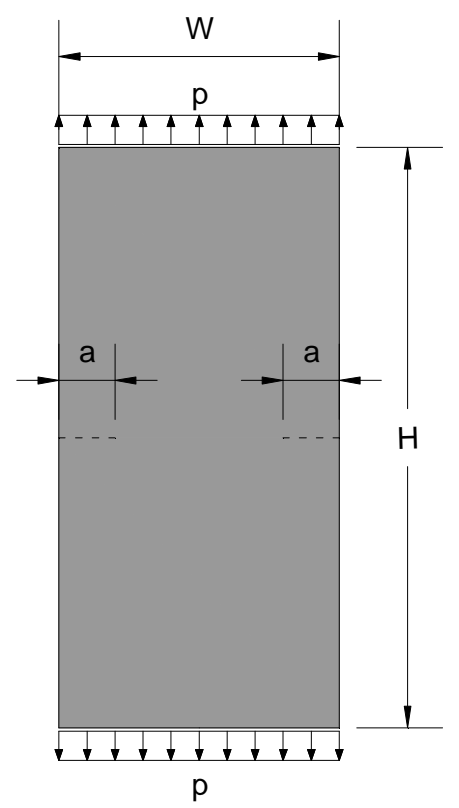

Figura 5.2 - Subproblema global $P_{G}^{(0)}$.

O objetivo dessa etapa é a descrição das tensões, genericamente indicadas por $\boldsymbol{t}_{\boldsymbol{G}}^{(\mathbf{0})}$, nas linhas que representam as posições das fissuras. As tensões determinadas na solução deste subproblema empregando certa discretização são aproximadas por uma combinação linear, utilizando funções (chamados aqui de termos de aproximação) adimensionalizadas pelo comprimento de cada fissura (Equação (5.1)).

$$
t_{G}^{(0)} \approx \sum_{j=1}^{J} b_{i, j} \cdot Q_{j}\left(\xi_{i} / a_{i}\right)
$$

Na expressão acima, $J$ é o número máximo de termos utilizados na combinação linear, $i$ é o índice que representa a fissura em análise, $j$ o índice que representa os termos de aproximação, $b_{i, j}$ são constantes e $Q_{j}\left(\xi_{i} / a_{i}\right)$ os termos de aproximação propriamente ditos, representados pela seguinte expressão:

$$
Q_{j}\left(\xi_{i} / a_{i}\right)=\left(\frac{\xi_{i}}{a_{i}}\right)^{j-1}
$$

Observe que as constantes $b_{i, j}$ são conhecidas nessa etapa. 


\section{3- 2o Subproblema}

O segundo é denominado de subproblema local $\left(P_{L}^{(k)}\right)$ e consiste na análise da fissura padrão contida em um meio infinito. O objetivo essencial é determinar o FIT. Nesse subproblema, as condições de contorno do problema original, tanto em força quanto em deslocamento, não são levadas em conta (Figura 5.3).

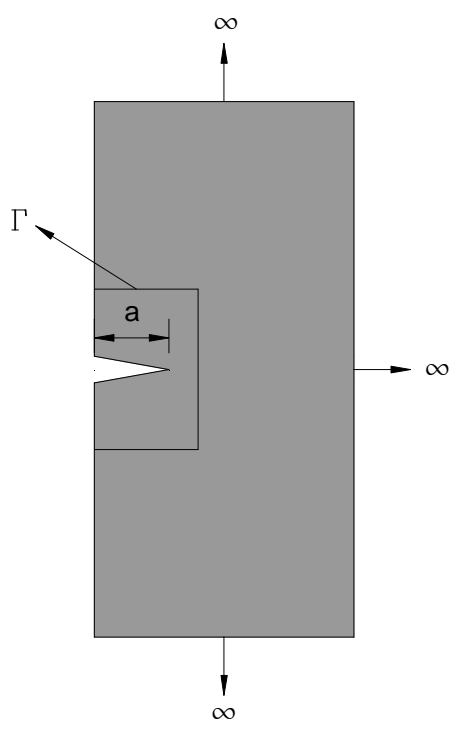

Figura 5.3 - Subproblema local $P_{L}^{(k)}$.

As mesmas bases de funções aproximativas empregadas para a aproximação de tensões do $P_{G}^{(0)}$ (Equação (5.2)) são utilizadas aqui, agora como carregamentos com valores máximos unitários aplicados nas faces da fissura. Para cada termo de aproximação aplicado, determinam-se os FIT e os deslocamentos e tensões em uma região arbitrária, chamada de contorno $\Gamma$. Essa região deve estar próxima à fissura e contorná-la completamente (Figura 5.3). As tensões, genericamente indicadas por $t_{L}^{(k)}$, são representadas por:

$$
t_{L}^{(k)}=-\sum_{j=1}^{J} \alpha_{j+(i-1) J} \cdot Q_{j}\left(\xi_{i} / a_{i}\right)
$$

Na expressão acima, $Q_{j}\left(\xi_{i} / a_{i}\right)$ é dado pela Equação (5.2). 
O valor máximo do índice $k$ é igual ao produto entre o número de fissuras diferentes do problema original $(I)$ e o número de termos utilizados para a aproximação de tensões $(J)$. Assim, os subproblemas $P_{L}^{(k)}$ são resolvidos $k$ vezes.

\section{4- 3o Subproblema}

O último subproblema é composto por subploblemas globais $\left(P_{G}^{(k)}\right)$ e tem por objetivo representar os efeitos das interações entre as fissuras. Nos $P_{G}^{(k)}$, utilizam-se o domínio do problema original com as condições de contorno em deslocamento, porém sem a presença das fissuras e sem os carregamentos externos.

Para simular os efeitos que uma fissura provoca sobre as demais são aplicados os deslocamentos obtidos no problema $P_{L}^{(k)}$ no mesmo contorno $\Gamma$ (Figura 5.4).

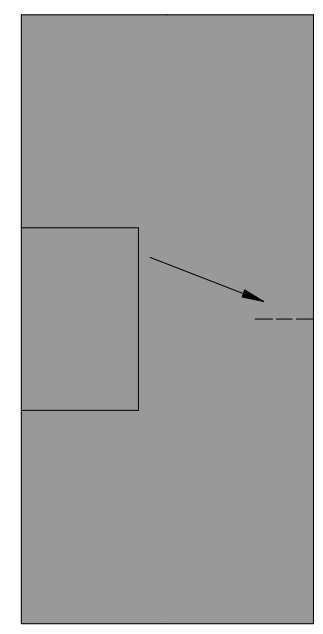

Figura 5.4 - Subproblema $\boldsymbol{P}_{G}^{(k)}$.

O objetivo desse subproblema é a determinação das tensões, genericamente indicadas por $\boldsymbol{t}_{G}^{(k)}$, nas linhas que representam as fissuras externas ao contorno $\Gamma$. As tensões naquelas linhas, determinadas na solução do problema com certa discretização, também são aproximadas pelos mesmos termos utilizados no $P_{G}^{(0)}$. As tensões $\boldsymbol{t}_{\boldsymbol{G}}^{(\boldsymbol{k})}$ são representadas por: 


$$
t_{G}^{(k)}=\sum_{j=1}^{J} c_{i, j}^{(k)} \cdot Q_{j}\left(\xi_{i} / a_{i}\right)
$$

Nesse subproblema, não se consideram os efeitos desses deslocamentos na fissura interna ao contorno $\Gamma$. Observa-se que a diferença entre o subproblema $P_{G}^{(0)}$ e $P_{G}^{(k)}$ está apenas no carregamento aplicado.

$\mathrm{Na}$ Equação (5.4), $c_{i, j}^{(k)}$ representa as constantes da combinação linear e $Q_{j}\left(\xi_{i} / a_{i}\right)$ é dado pela Equação (5.2).

Observa-se ainda que as constantes $c_{i, j}^{(k)}$ são conhecidas nessa etapa e que, assim como nos subproblemas $P_{L}^{(k)}$, os $P_{G}^{(k)}$ são resolvidos $k$ vezes.

\section{5- Solução numérica}

A representação matemática para a sobreposição pode ser dada pela seguinte equação:

$$
P_{G}=P_{G}^{(0)}+\sum_{k=1}^{M} \alpha_{k} \cdot P_{L}^{(k)}+\sum_{k=1}^{M} \alpha_{k} \cdot P_{G}^{(k)}
$$

O símbolo $\alpha_{k}$ representa os fatores de escala utilizados na determinação dos FIT finais das fissuras e $M$ representa o número máximo de problemas $P_{L}^{(k)}$ e $P_{G}^{(k)}$, ou seja:

$$
M=I . J
$$

Na equação acima, $I$ representa o número de fissuras diferentes e $J$ representa o número de termos de aproximação.

$\mathrm{Na}$ Equação (5.5), os valores de $P_{L}^{(k)}$ no domínio externo ao contorno $\Gamma$ são desconsiderados. Ao contrário, no $P_{G}^{(k)}$ os valores desconsiderados são os da região interna ao contorno $\Gamma$.

A montagem do sistema para o cálculo dos parâmetros $\alpha$ decorre da imposição em forma fraca de tensões nulas nas faces das fissuras. Essa imposição é realizada em forma de resíduos ponderados (Equação (5.7)). 


$$
\int_{0}^{a_{i}} t^{T} Q_{j 2}\left(\xi_{i} / a_{i}\right) d \xi=0 \quad \text { com } j 2=1, \ldots, J
$$

onde:

$$
\begin{gathered}
\boldsymbol{t}=\boldsymbol{t}_{\boldsymbol{G}}^{(0)}+\boldsymbol{t}_{\boldsymbol{L}}^{(k)}+\boldsymbol{t}_{\boldsymbol{G}}^{(k)} \\
Q_{j 2}\left(\xi_{i} / a_{i}\right)=\left(\frac{\xi_{i}}{a_{i}}\right)^{j-1}
\end{gathered}
$$

sendo que $\boldsymbol{t}$ representa a soma das tensões nas faces das fissuras de cada um dos três subproblemas (Equação (5.8)). O índice $i$ faz referência à fissura, $Q_{j 2}$ é a função ponderadora (Equação (5.9)), função do comprimento da fissura $\left(a_{i}\right)$ e da coordenada $\xi$ ao longo da fissura. A variável $j$ da Equação (5.9) representa o número do termo de aproximação de tensão utilizado.

Observando as Equações (5.4) e (5.5), obtém-se a expressão final para $t_{G}^{(k)}$ :

$$
t_{G}^{(k)}=\sum_{j=1}^{J} \sum_{k=1}^{M} \alpha_{k} \cdot c_{i, j}^{(k)} \cdot Q_{j}\left(\xi_{i} / a_{i}\right)
$$

sendo $\alpha$ o parâmetro a ser determinado (observe que ele aparece tanto em $P_{L}^{(k)}$ quanto em $P_{G}^{(k)}$, pois eles são dependentes).

As variáveis $t_{G}^{(0)}$ e $t_{L}^{(k)}$ são representadas pelas Equações (5.1), (5.3).

Substituindo as Equações (5.1), (5.3) e (5.10) em (5.8) e o resultado disso em (5.7) temse:

$$
\int_{0}^{a_{i}} \sum_{j=1}^{J}\left[b_{i, j}-\alpha_{j+(i-1) J}+\sum_{k=1}^{M} \alpha_{k} \cdot c_{i, j}^{(k)}\right] Q_{j}\left(\xi_{i} / a_{i}\right) \cdot Q_{j 2}\left(\xi_{i} / a_{i}\right) d \xi=0
$$

Isolando os termos dependentes de $\alpha$ da equação acima obtêm-se o seguinte sistema linear:

$$
[I G] \cdot\{\alpha\}=\{r\}
$$


Onde $[I G]$ é chamada de matriz de influência geral, já que mantém os termos responsáveis pela interação entre fissuras. O vetor $\alpha$ fornece os fatores de escala responsáveis para encontrar os FIT finais para cada fissura:

$$
K^{(i)}(J)=\sum_{j=1}^{J} \alpha_{j+(i-1) \cdot J} \cdot K_{j}^{(j)}
$$

sendo $K_{j}^{(j)}$ os FIT calculados no subproblema $P_{L}^{(k)}$ para cada termo e $K^{(i)}(J)$ os FIT finais para cada fissura.

A dedução do sistema linear dado pela Equação (5.12) pode ser encontrada no Anexo (B) retirado do trabalho de Alves (2010).

Um ponto a ser observado é que os problemas $P_{G}^{(0)}$ e $P_{L}^{(k)}$ são independentes entre si, assim como os problemas $k$ do $P_{L}^{(k)}$ e do $P_{G}^{(k)}$. Portanto esse método permite explorar o recurso da paralelização, diminuindo, assim, o custo computacional. Além disso, em problemas cujas fissuras são iguais, só é necessário realizar o problema $P_{L}^{(k)}$ uma vez, pois o resultado é o mesmo para todas as fissuras. 


\section{6- Resultados}

\section{1- Considerações iniciais}

Neste capítulo apresentam-se quatro aplicações elaboradas a fim de verificar o desempenho da formulação híbrido-Trefftz e do MEFG aplicados a sólidos contendo fissuras. Inicialmente, foi analisado um problema contendo uma única fissura modelado sem o uso do Método da Partição. Neste caso, o intuito é o de validar cada uma das formulações numéricas. Em seguida, as formulações foram aplicadas no Método da Partição em problemas contendo duas fissuras. A grandeza de interesse para as análises é o FIT do modo I de abertura da fissura. Os valores encontrados foram comparados com os analíticos ou com valores de referência obtidos mediante o MEF convencional pelo software ANSYS ${ }^{\circledR}$. Para ambas formulações, as redes foram geradas com o auxílio do software ANSYS ${ }^{\circledR}$.

Observa-se que nos problemas contendo duas fissuras simétricas o subproblema local foi resolvido para uma das fissuras, já que os dados obtidos na análise são os mesmos para a outra fissura. Para todos os casos, o módulo de elasticidade transversal e o coeficiente de Poisson valem, respectivamente, $G=1,0$ e $v=0,3$.

No uso da formulação híbrido-Trefftz a aproximação dos campos de deslocamentos no contorno do elemento foi obtida mediante a utilização de quatro bases de aproximação: $\beta_{0}$, $\beta_{1}, \beta_{2}$ e $\beta_{3}$. Tais bases foram definidas no capítulo 2. Vale destacar que em todos os problemas a simetria horizontal foi explorada ao utilizar esta formulação.

Para a análise com o MEFG foi utilizado o elemento isoparamétrico quadrilateral com quatro nós representado na Figura 4.3. Como a solução para o problema contendo uma ou mais fissuras possui uma parcela regular (no entorno da ponta da fissura) e uma parcela irregular (predominante na ponta da fissura), o enriquecimento nodal foi feito com funções polinomiais e funções que representam a solução analítica de problemas da mecânica da fratura. As funções formadas pela solução analítica serão representadas pela letra $F$ seguido de $x$ se a função for definida na direção de $\mathrm{x}$ e seguindo de $y$ se a função for definida na direção de y. Elas podem ser encontradas no trabalho de Alves (2010). Ao contrário da 
aplicação da formulação híbrido-Trefftz, ao empregar o MEFG não foi explorada a simetria horizontal dos problemas. Isso porque as funções enriquecedoras utilizadas neste trabalho não são do tipo "bolha", ou seja, os parâmetros adicionais a elas associados não valem zero nos nós e destroem o significado dos parâmetros nodais originais. Portanto, a imposição da condição de contorno homogênea requer uma relação de dependência entre os parâmetros, de implementação computacional mais trabalhosa. Assim, para evitar essa dificuldade, optou-se por realizar a análise de todo o domínio do sólido.

Para a resolução dos problemas foi admitido um regime de linearidade física e geométrica, bem como a hipótese de forças volúmicas nulas. Para simplificar o procedimento, as unidades de cada grandeza foram omitidas.

A extração do FIT para os problemas analisados com a formulação híbrido-Trefftz é obtida com base no campo de tensões próximo a ponta da fissura. Assim, toma-se como referência uma faixa a partir da ponta da fissura até uma distância igual a $10 \%$ do seu comprimento. O valor do FIT é dado pela Equação (6.1).

$$
F I T=\lim _{r \rightarrow 0} \sqrt{2 \pi r} \sigma_{i}
$$

Sendo:

- $\quad r$ a distância dos pontos considerados até a ponta da fissura;

- $\sigma_{i}$ o valor da tensão no ponto considerado.

Já para os problemas analisados com o MEFG a extração dessa mesma grandeza foi obtida pela técnica de correlação dos deslocamentos (SOUZA, 2008 e LEONEL, 2006). Nessa técnica foram escolhidos pontos das faces da fissura situados a partir da ponta a uma distância de $10 \%$ do seu comprimento. O deslocamento vertical desses pontos determina o valor do FIT pela Equação (6.2).

$$
F I T=\lim _{r \rightarrow 0} \frac{G}{\kappa+1} \sqrt{\frac{2 \pi}{r}} \Delta \varsigma
$$

Sendo:

- $G=\frac{E}{2(1+v)}$ o módulo de elasticidade transversal; 
- $\quad E, v$ o módulo de elasticidade e o coeficiente de Poisson respectivamente;

- $\kappa=3-4 v$ para o Estado Plano de Deformações (EPD) e $\kappa=\frac{3-v}{1+v}$ para o Estado Plano de Tensões (EPT);

- $\quad r$ a distância dos pontos de referência até a ponta da fissura;

- $\Delta \varsigma$ o deslocamento entre as duas faces da fissura dado pelos pontos considerados.

Nos exemplos, foi adotada uma rede de elementos finitos fixa para cada subproblema local. As redes apresentadas foram as que proporcionaram os melhores resultados considerando um erro relativo em torno de $1 \%$. Destaca-se ainda que o comprimento da fissura é invariável, ou seja, sua propagação não foi analisada.

\section{2- 1ㅇ Problema}

O primeiro problema trata da análise de uma chapa contendo uma fissura horizontal submetida a um carregamento constante em suas bordas (Figura 6.1).

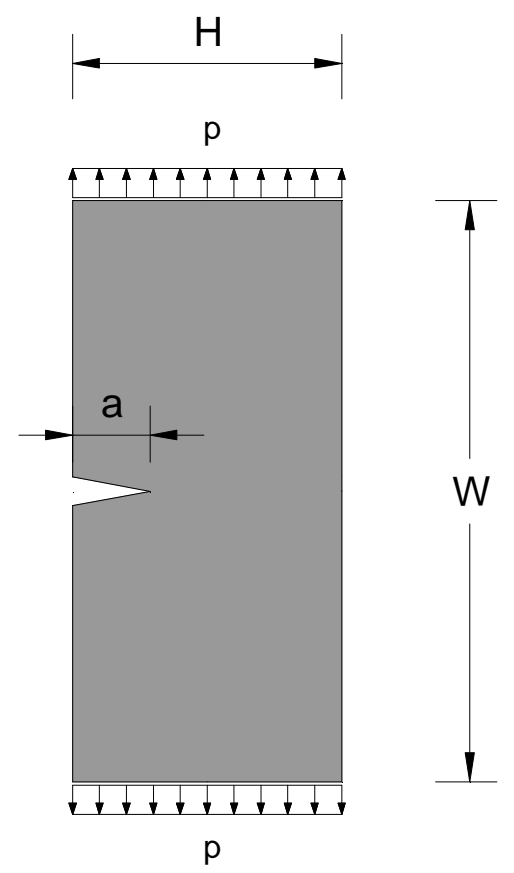

Figura 6.1 - Chapa com uma fissura e carregamento constante. 
No dimensionamento, adotou-se a largura $H$ da chapa igual a 10 , o comprimento $W$ igual a 20 e o comprimento a da fissura igual a 1 . O carregamento uniformemente distribuído $p$ vale 1 .

O valor analítico do FIT desse problema vale 2,098 (ALVES, 2010).

Para a análise do problema não foi utilizado o Método da Partição, pois seu uso não é necessário em problemas contendo uma só fissura. Além disso, o objetivo neste caso é o de avaliar o desempenho das formulações numéricas híbrido-Trefftz e MEFG.

\subsection{1 - Análise utilizando a formulação híbrido-Trefftz}

Para a modelagem foi utilizada a simetria horizontal do problema (Figura 6.2).

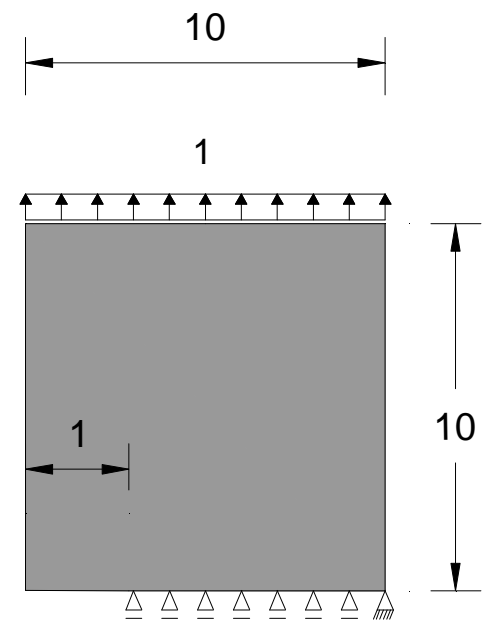

Figura 6.2 - Modelo do $1^{\circ}$ problema utilizando a formulação híbrido-Trefftz.

A rede que compõe a discretização do problema é ilustrada na Figura 6.3 e o detalhe da região próximo à fissura é dado pela Figura 6.4. 


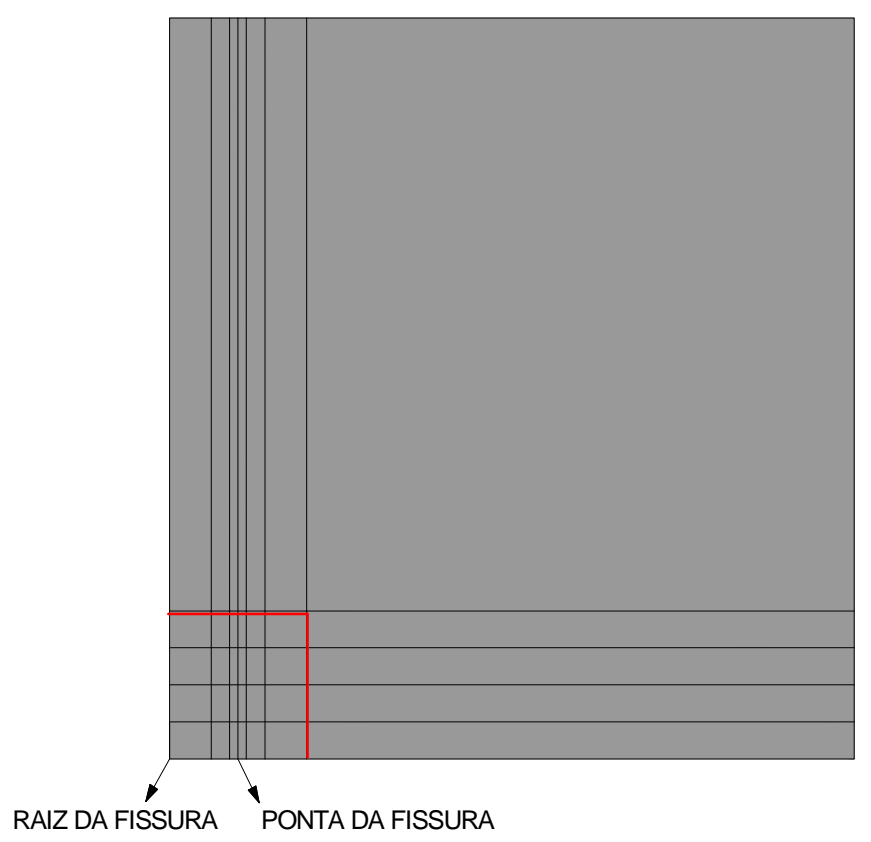

Figura 6.3 - Rede do $1^{\circ}$ problema utilizando a formulação híbrido-Trefftz.

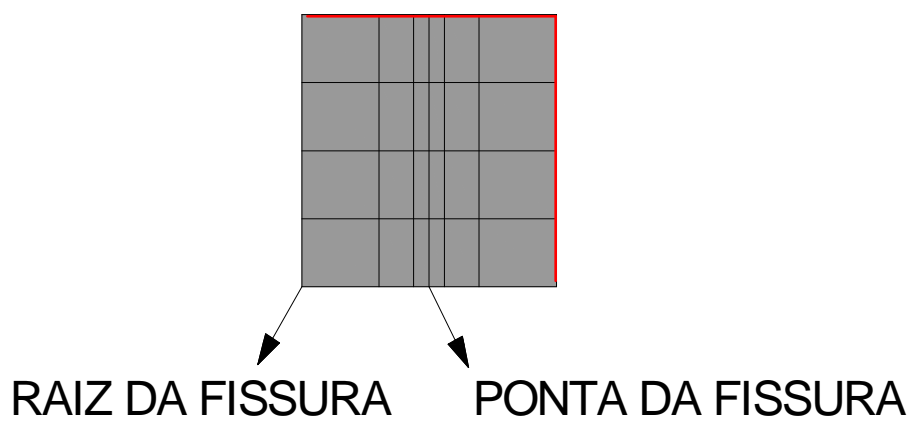

Figura 6.4 - Detalhe da região próximo à fissura.

A chapa foi discretizada utilizando o elemento finito híbrido-Trefftz descrito neste trabalho. A rede contém um total de 35 elementos e 48 nós, sendo que todos os contornos foram enriquecidos, exceto aqueles vinculados.

Os resultados obtidos são apresentados nas Tabela 6.1 a Tabela 6.4. Inicialmente é apresentado o resultado para a análise sem enriquecimento (base $\beta_{0}$ ). Em seguida são mostrados os resultados para os enriquecimentos utilizando as bases $\beta_{1}, \beta_{2}$ e $\beta_{3}$ variando a dimensão das bases enriquecedoras. 
Tabela 6.1 - Resultados do FIT sem enriquecimento

\begin{tabular}{c|c|c|c|c}
\hline \multicolumn{5}{c}{ SEM ENRIQUECIMENTO $\left(\beta_{0}\right)$} \\
\hline $\begin{array}{c}\text { Dimensão da } \\
\text { base } \\
\text { enriquecedora }\end{array}$ & FIT final & $\begin{array}{c}\text { FIT } \\
\text { analítico }\end{array}$ & $\begin{array}{c}\text { Erro relativo } \\
(\%)\end{array}$ & $\begin{array}{c}\text { Graus de } \\
\text { liberdade } \\
\text { totais }\end{array}$ \\
\hline 0 & 0,580 & 2,098 & 72,35 & 1735 \\
\hline
\end{tabular}

Tabela 6.2 - Resultados do FIT com enriquecimento hierárquico

\begin{tabular}{c|c|c|c|c}
\hline \multicolumn{5}{c}{ ENRIQUECIMENTO HIERÁRQUICO $\left(\beta_{1}\right)$} \\
\hline $\begin{array}{c}\text { Dimensão da } \\
\text { base } \\
\text { enriquecedora }\end{array}$ & FIT final & $\begin{array}{c}\text { FIT } \\
\text { analítico }\end{array}$ & $\begin{array}{c}\text { Erro } \\
\text { relativo }(\%)\end{array}$ & $\begin{array}{c}\text { Graus de } \\
\text { liberdade } \\
\text { totais }\end{array}$ \\
\hline 1 & 1,125 & 2,098 & 46,38 & 1891 \\
\hline 2 & 1,519 & 2,098 & 27,60 & 2047 \\
\hline 3 & 1,821 & 2,098 & 13,20 & 2203 \\
\hline 4 & 2,129 & 2,098 & 1,48 & 2359 \\
\hline 5 & 2,411 & 2,098 & 14,92 & 2515 \\
\hline 6 & 2,559 & 2,098 & 21,97 & 2671 \\
\hline
\end{tabular}

Tabela 6.3 - Resultados do FIT com enriquecimento não-hierárquico

\begin{tabular}{c|c|c|c|c}
\hline \multicolumn{4}{c}{ ENRIQUECIMENTO NÃO-HIERÁRQUICO $\left(\beta_{2}\right)$} \\
\hline $\begin{array}{c}\text { Dimensão da } \\
\text { base } \\
\text { enriquecedora }\end{array}$ & FIT final & $\begin{array}{c}\text { FIT } \\
\text { analítico }\end{array}$ & $\begin{array}{c}\text { Erro } \\
\text { relativo }(\%)\end{array}$ & $\begin{array}{c}\text { Graus de } \\
\text { liberdade } \\
\text { totais }\end{array}$ \\
\hline 1 & 0,777 & 2,098 & 62,96 & 1891 \\
\hline 2 & 1,539 & 2,098 & 26,64 & 2047 \\
\hline 3 & 1,821 & 2,098 & 13,20 & 2203 \\
\hline 4 & 1,973 & 2,098 & 5,96 & 2359 \\
\hline 5 & 2,288 & 2,098 & 9,06 & 2515 \\
\hline 6 & 2,535 & 2,098 & 20,83 & 2671 \\
\hline
\end{tabular}


Tabela 6.4 - Resultados do FIT com enriquecimento trigonométrico

\begin{tabular}{c|c|c|c|c}
\hline \multicolumn{5}{c}{ ENRIQUECIMENTO TRIGONOMÉTRICO $\left(\beta_{3}\right)$} \\
\hline $\begin{array}{c}\text { Dimensão da } \\
\text { base } \\
\text { enriquecedora }\end{array}$ & FIT final & $\begin{array}{c}\text { FIT } \\
\text { referência }\end{array}$ & $\begin{array}{c}\text { Erro relativo } \\
(\%)\end{array}$ & $\begin{array}{c}\text { Graus de } \\
\text { liberdade } \\
\text { totais }\end{array}$ \\
\hline 1 & 0,931 & 2,098 & 55,62 & 1891 \\
\hline 2 & 1,403 & 2,098 & 33,13 & 2047 \\
\hline 3 & 1,752 & 2,098 & 16,49 & 2203 \\
\hline 4 & 2,077 & 2,098 & 1,00 & 2359 \\
\hline 5 & 2,352 & 2,098 & 12,11 & 2515 \\
\hline 6 & 2,523 & 2,098 & 20,26 & 2671 \\
\hline
\end{tabular}

Analisando os dados das tabelas acima, verifica-se que o menor erro relativo é atingido quando se utiliza o enriquecimento trigonométrico (Tabela 6.4) com uma dimensão da base enriquecedora igual a quatro.

Comparando os resultados da Tabela 6.2 com a Tabela 6.4, nota-se que os valores dos erros relativos estão bastante próximos. Já o enriquecimento com base não-hierárquica (Tabela 6.3) apresentam valores de erros relativos muito altos. Finalmente, comparando os resultados da Tabela $6.1 \mathrm{com}$ as demais tabelas, verifica-se uma grande queda do erro relativo, comprovando a melhora na aproximação da solução ao utilizar o enriquecimento.

Observa-se que ao utilizar uma dimensão da base enriquecedora maior que quatro, o erro relativo volta a subir. Isso pode estar relacionado ao não atendimento da condição (3.45) causa instabilidade na aproximação da solução.

Para ilustrar a obtenção do FIT, considere o gráfico $F I T_{i}$ x $r$ para o caso com enriquecimento utilizando a base $\beta_{3}$ e com a dimensão da base enriquecedora igual a quatro (Figura 6.5). No gráfico, $F I T_{i}$ representa o valor $\sqrt{2 \pi r} \sigma_{i}$ no ponto "i" considerado e $r$ representa a distância entre a ponta da fissura e o ponto considerado para a obtenção do FIT. 


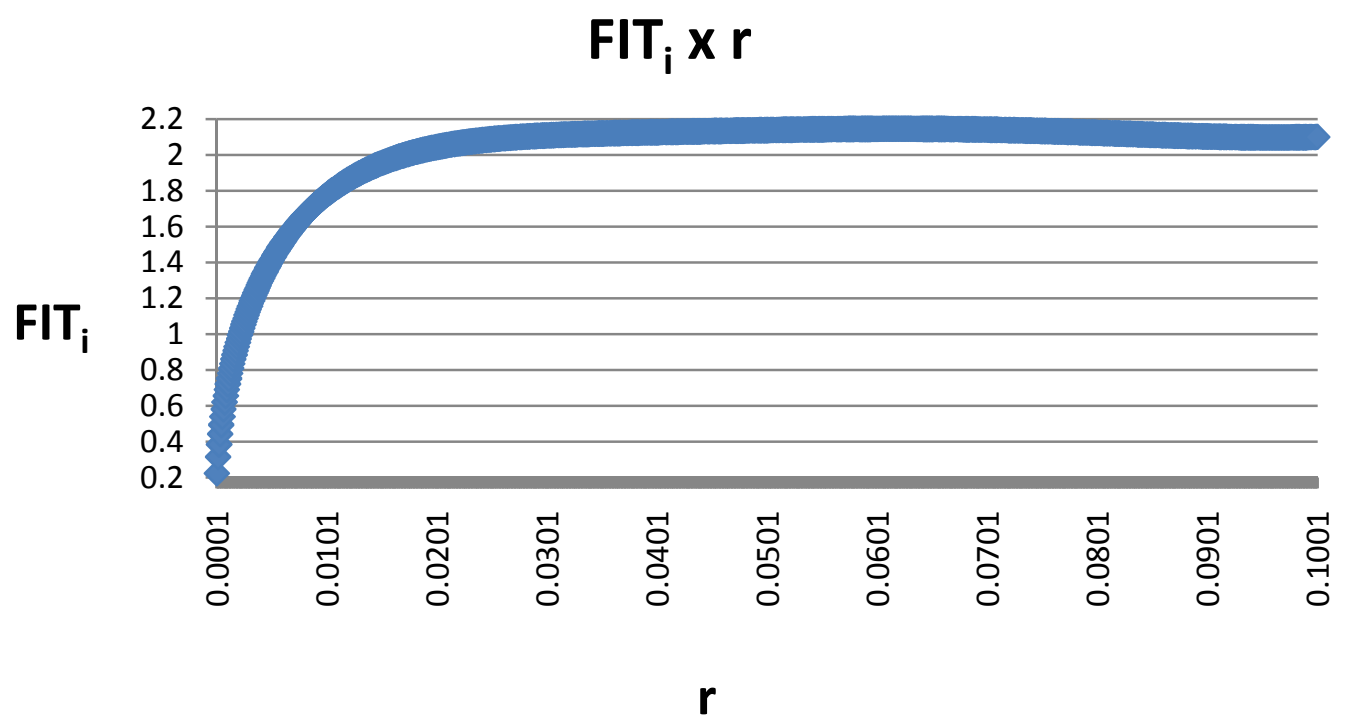

Figura 6.5 - Gráfico do FIT $_{\mathrm{i}}$ x r

Percebe-se que os pontos mais próximos da ponta da fissura sofrem uma grande perturbação com relação aos valores de $\mathrm{FIT}_{\mathrm{i}}$, pois o valor de $r$ tende a zero. Em pontos afastados, também ocorre uma perturbação.

Portanto, para a extração do FIT, foi feita uma média utilizando mínimos quadrados no trecho entre $r=0,02$ e $r=0,08$, já que nessa faixa observamos uma estabilidade da curva. Ao todo, foram coletados valores de FIT $_{i}$ em 600 pontos (para todos os casos, a quantidade desses pontos foi arbitrário), sendo a distância entre eles igual a 0,001.

\subsection{2 - Análise utilizando o MEFG}

Para a modelagem não foi utilizada a simetria horizontal do problema. Os dados geométricos do problema estão mostrados na Figura 6.6. 

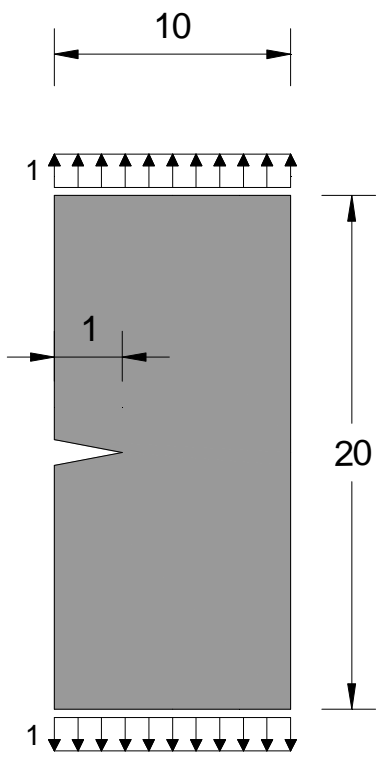

Figura 6.6 - Modelo do $1^{\circ}$ problema utilizando o MEFG

A rede utilizada no problema é ilustrada pela Figura 6.7 e o detalhe da região próximo à fissura é dado pela Figura 6.8.

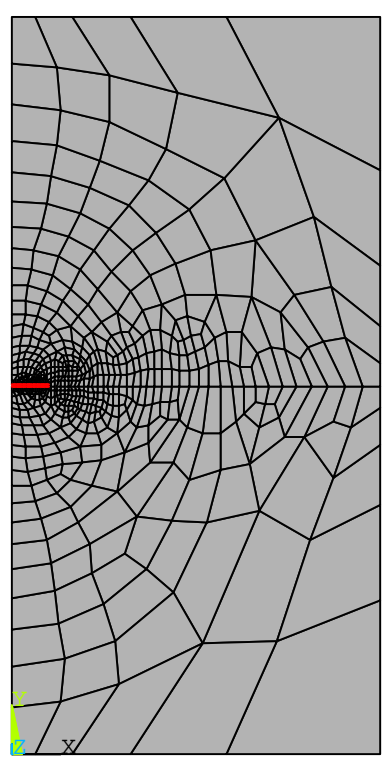

Figura 6.7 - Rede do $1^{\circ}$ problema utilizando o MEFG 


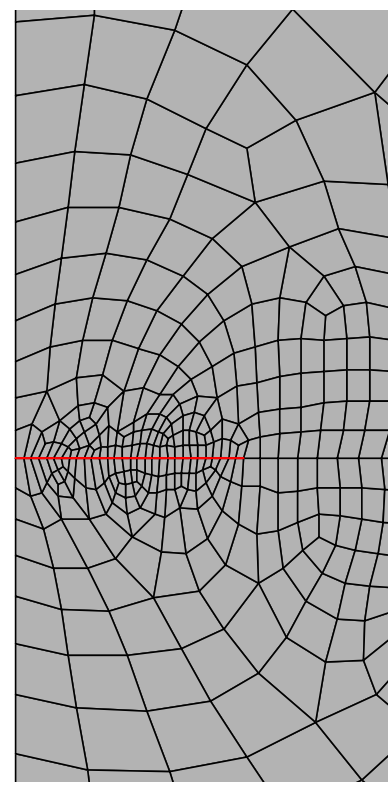

Figura 6.8 - Detalhe da região próximo à fissura

Na Figura 6.7 e Figura 6.8 a linha vermelha indica a fissura. A rede contém um total de 717 nós e 656 elementos. Todos os nós foram enriquecidos em ambas as direções (x e y), exceto aqueles vinculados. As funções enriquecedoras utilizadas são: $\mathrm{x}, \mathrm{x}^{2}, \mathrm{x}^{3}, \mathrm{x}^{4}, \mathrm{x}^{5}, F x$ na direção x e y, $\mathrm{y}^{2}, \mathrm{y}^{3}, \mathrm{y}^{4}, \mathrm{y}^{5}, F y$ na direção y. O resultado obtido com todos os enriquecimentos aplicados simultaneamente é apresentado na Tabela 6.5 .

Tabela 6.5 - Resultados do FIT utilizando o MEFG

\begin{tabular}{c|c|c|c}
\hline $\begin{array}{c}\text { FIT } \\
\text { obtido }\end{array}$ & FIT referência & $\begin{array}{c}\text { Erro relativo } \\
(\%)\end{array}$ & $\begin{array}{c}\text { Graus de } \\
\text { liberdades } \\
\text { totais }\end{array}$ \\
\hline 2,064 & 2,098 & 1,62 & 9870 \\
\hline
\end{tabular}

\section{3- 2을 Problema}

O segundo problema enfatiza a análise de três casos dos subproblemas locais $\left(P_{L}^{(k)}\right)$ :

- Carregamento constante aplicado nas faces de uma fissura de comprimento "a" imersa num meio infinito; 
- Carregamento linear aplicado nas faces de uma fissura de comprimento "a" imersa num meio infinito;

- Carregamento quadrático aplicado nas faces de uma fissura de comprimento "a" imersa num meio infinito.

O valor do FIT do primeiro caso foi comparado com o valor analítico. Os demais foram comparados com os valores de referência. Os valores de referência para o FIT foram retirados do trabalho de Alves (2010). Naquele trabalho, para a modelagem dos subproblemas $P_{L}^{(k)}$ foram utilizados elementos finitos quadrilaterais de oito nós (deslocamentos em duas direções por nó), aproveitando a simetria horizontal do problema. A rede foi composta de 25107 nós e 8310 elementos, num total de 50214 graus de liberdade. A análise foi realizada pelo MEF clássico.

Nota-se que a ênfase é sobre o campo local de tensões, pois, ao utilizar o Princípio de Bueckner, apenas nas vizinhanças da ponta da fissura é que se observa a equivalência de distribuição de tensões entre os casos em que o carregamento é aplicado nas faces da fissura e aplicado nas bordas da chapa.

\subsection{1 - Análise utilizando a formulação híbrido-Trefftz}

Para a modelagem dos três casos foi explorada a simetria horizontal do problema. Adotou-se a dimensão infinita da chapa como uma dimensão igual ao da chapa do $3^{\circ}$ problema (que será visto adiante), pois nele serão utilizados esses mesmos subproblemas $P_{L}^{(k)}$. Essa coincidência de dimensões não trará prejuízo ao resultado final já que se trata de um problema contendo poucas fissuras.

A rede de elementos finitos híbrido-Trefftz utilizada nos três casos pode ser visualizada na Figura 6.9. Ela contém um total de 120 nós e 99 elementos. O interior ampliado do contorno gama pode ser visto na Figura 6.10. Todos os trechos da rede foram enriquecidos, com exceção daqueles que possuem nós vinculados. 


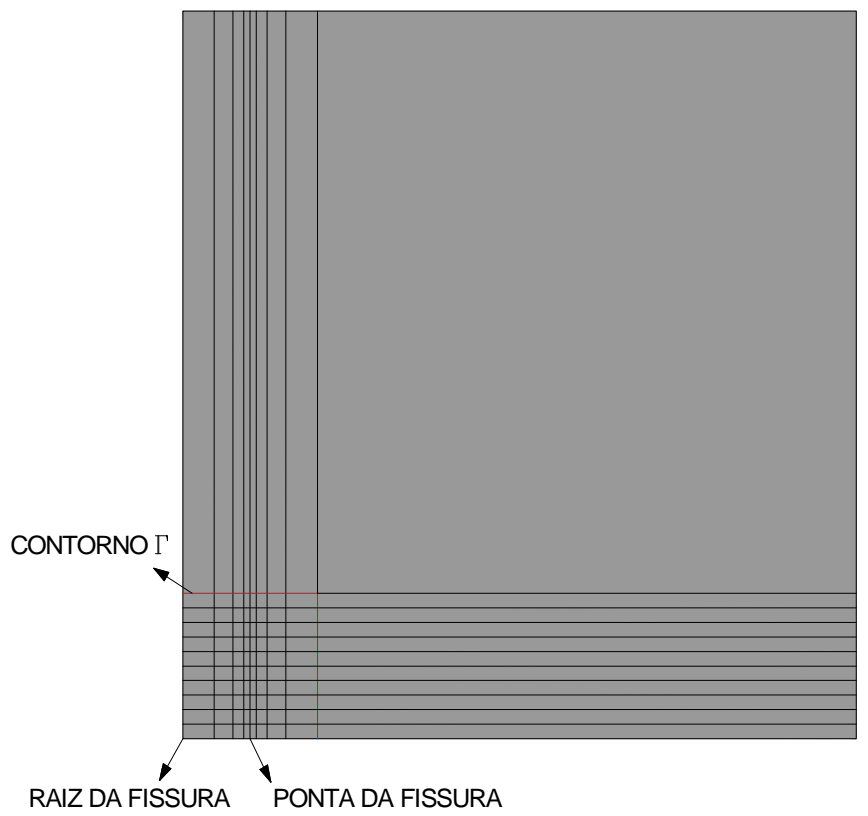

Figura 6.9 - Rede de elementos finitos híbrido-Trefftz do $2^{\circ}$ problema

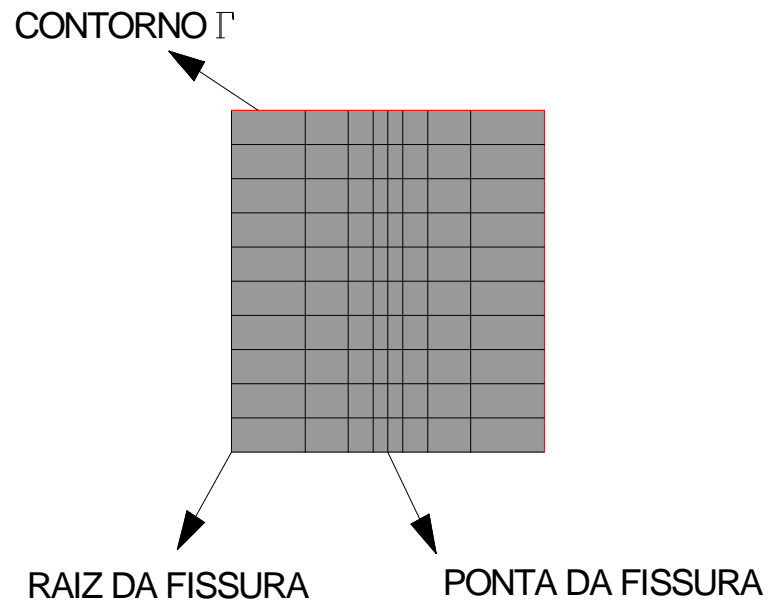

Figura 6.10 - Detalhe da região interna ao contorno gama

A seguir são detalhados cada um dos três casos do subproblema $P_{L}^{(k)}$.

\subsubsection{1- Carregamento uniforme aplicado na face da fissura}

Como comentado, o primeiro subproblema local é mostrado na Figura 6.11. 


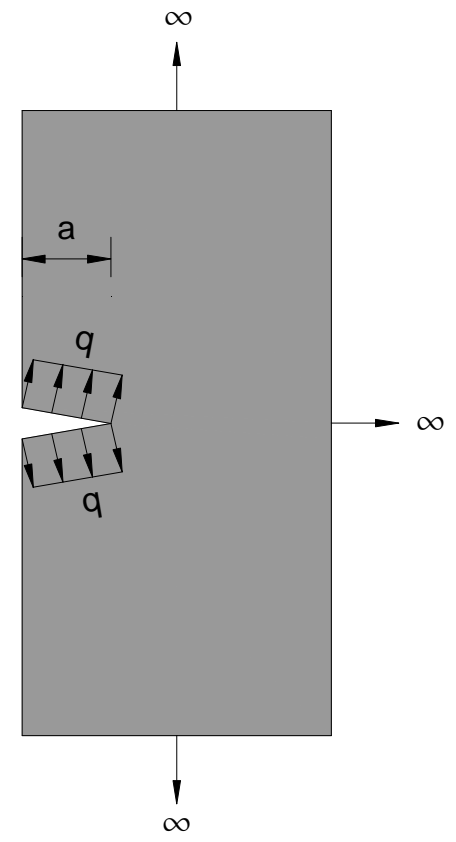

Figura 6.11 - Primeiro subproblema local.

O problema foi modelado explorando-se a simetria de acordo com a Figura 6.12:

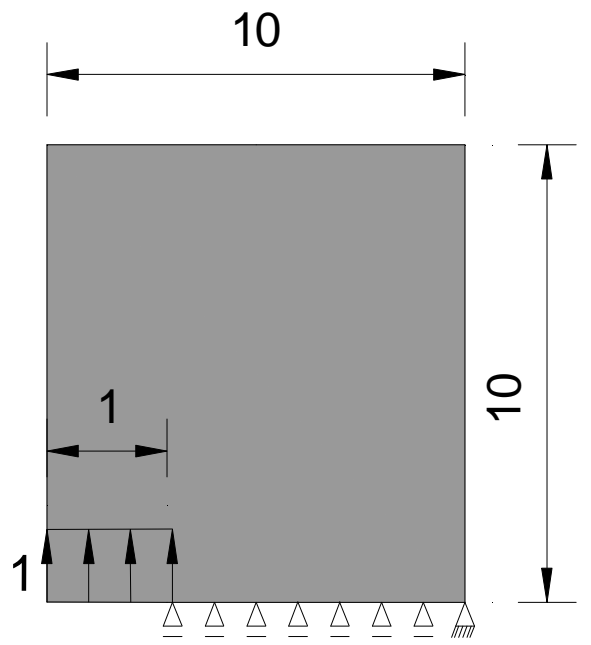

Figura 6.12 - Modelo para o primeiro subproblema local

A curva do $F I T_{i}$ x $r$ para o caso em que é utilizado o enriquecimento hierárquico com uma dimensão da base enriquecedora igual a cinco é apresentada na figura abaixo: 


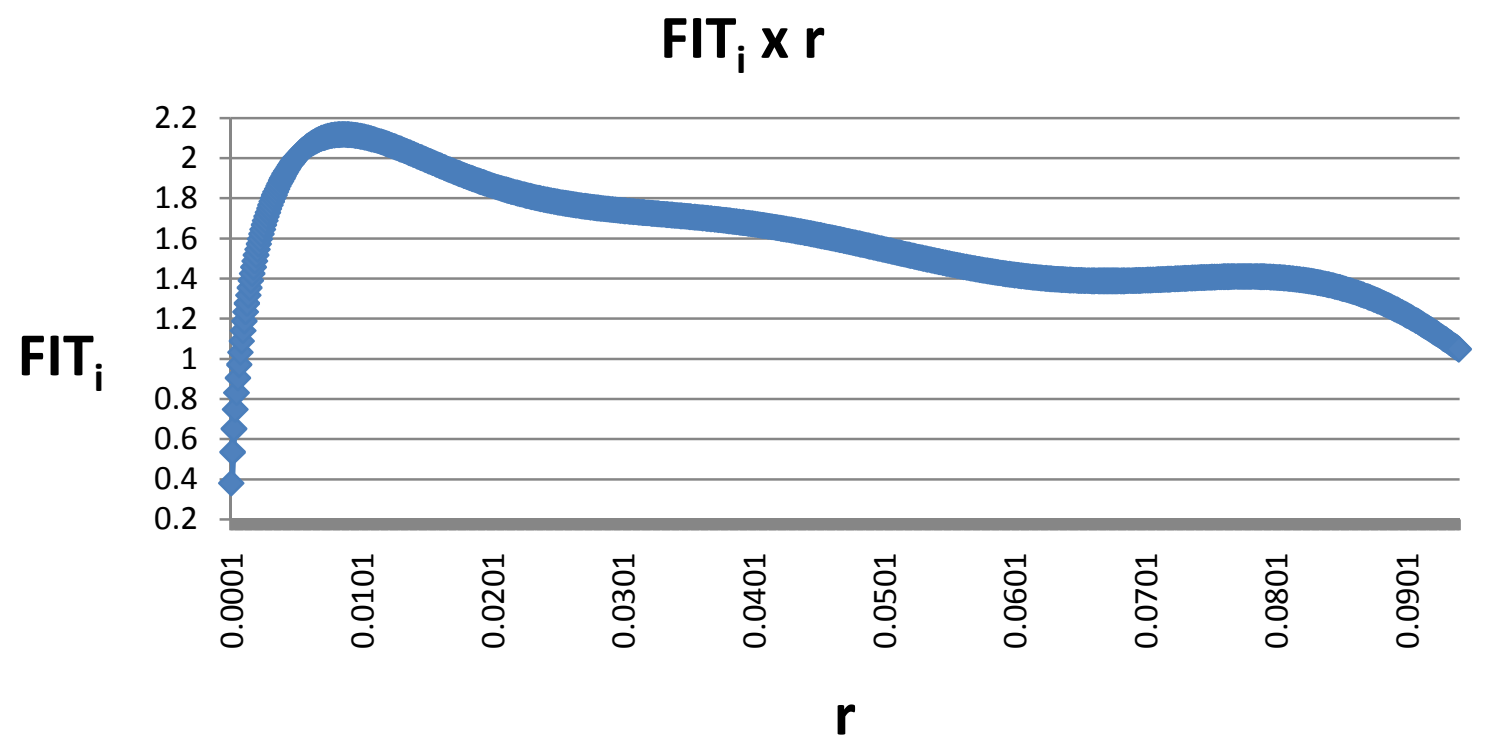

Figura 6.13 - Curva de FIT $_{i}$ x r para o primeiro subproblema local

Assim como na Figura 6.5, percebe-se do gráfico acima que os pontos mais próximos da ponta da fissura sofrem uma grande perturbação com relação aos valores de FIT $_{\mathrm{i}}$, pois o valor de $r$ tende a zero. Em pontos afastados, pode-se também perceber uma perturbação.

Portanto, para a extração do FIT, também foi calculada uma média utilizando mínimos quadrados no trecho entre $\mathrm{r}=0,01$ e $\mathrm{r}=0,08$, já que nessa faixa observamos uma estabilidade da curva. Ao todo, foram coletados valores de FIT $_{\mathrm{i}}$ em 700 pontos, sendo a distância entre eles igual a 0,001 . O valor do FIT, bem como a comparação com o valor analítico, é dado pelas Tabela 6.6 a Tabela 6.9:

Tabela 6.6 - Resultados do FIT sem enriquecimento

\begin{tabular}{c|c|c|c|c}
\hline \multicolumn{5}{c}{ SEM ENRIQUECIMENTO $\left(\beta_{0}\right)$} \\
\hline $\begin{array}{c}\text { Dimensão da } \\
\text { base } \\
\text { enriquecedora }\end{array}$ & FIT final & $\begin{array}{c}\text { FIT } \\
\text { referência }\end{array}$ & $\begin{array}{c}\text { Erro relativo } \\
(\%)\end{array}$ & $\begin{array}{c}\text { Graus de } \\
\text { liberdade } \\
\text { totais }\end{array}$ \\
\hline 0 & 0,634 & 2,098 & 69,78 & 1735 \\
\hline
\end{tabular}


Tabela 6.7 - Resultados do FIT com enriquecimento hierárquico

\begin{tabular}{c|c|c|c|c}
\hline \multicolumn{5}{c}{ ENRIQUECIMENTO HIERÁRQUICO $\left(\beta_{1}\right)$} \\
\hline $\begin{array}{c}\text { Dimensão da } \\
\text { base } \\
\text { enriquecedora }\end{array}$ & FIT final & $\begin{array}{c}\text { FIT } \\
\text { referência }\end{array}$ & $\begin{array}{c}\text { Erro } \\
\text { relativo } \\
(\%)\end{array}$ & $\begin{array}{c}\text { Graus de } \\
\text { liberdade } \\
\text { totais }\end{array}$ \\
\hline 1 & 1,278 & 2,098 & 39,08 & 1891 \\
\hline 2 & 1,642 & 2,098 & 21,73 & 2047 \\
\hline 3 & 1,800 & 2,098 & 14,20 & 2203 \\
\hline 4 & 1,929 & 2,098 & 8,06 & 2359 \\
\hline 5 & 2,101 & 2,098 & 0,14 & 2515 \\
\hline 6 & 2,226 & 2,098 & 6,10 & 2671 \\
\hline
\end{tabular}

Tabela 6.8 - Resultados do FIT com enriquecimento não-hierárquico

\begin{tabular}{c|c|c|c|c}
\hline \multicolumn{4}{c}{ ENRIQUECIMENTO NÃO-HIERÁRQUICO $\left(\beta_{2}\right)$} \\
\hline $\begin{array}{c}\text { Dimensão da } \\
\text { base } \\
\text { enriquecedora }\end{array}$ & FIT final & $\begin{array}{c}\text { FIT } \\
\text { referência }\end{array}$ & $\begin{array}{c}\text { Erro } \\
\text { relativo } \\
(\%)\end{array}$ & $\begin{array}{c}\text { Graus de } \\
\text { liberdade } \\
\text { totais }\end{array}$ \\
\hline 1 & 1,089 & 2,098 & 48,09 & 1891 \\
\hline 2 & 1,517 & 2,098 & 27,69 & 2047 \\
\hline 3 & 1,800 & 2,098 & 14,20 & 2203 \\
\hline 4 & 1,846 & 2,098 & 12,01 & 2359 \\
\hline 5 & 2,051 & 2,098 & 2,24 & 2515 \\
\hline 6 & 2,203 & 2,098 & 5,00 & 2671 \\
\hline
\end{tabular}

Tabela 6.9 - Resultados do FIT com enriquecimento trigonométrico

\begin{tabular}{c|c|c|c|c}
\hline \multicolumn{5}{c}{ ENRIQUECIMENTO TRIGONOMÉTRICO $\left(\beta_{3}\right)$} \\
\hline $\begin{array}{c}\text { Dimensão da } \\
\text { base } \\
\text { enriquecedora }\end{array}$ & FIT final & $\begin{array}{c}\text { FIT } \\
\text { referência }\end{array}$ & $\begin{array}{c}\text { Erro relativo } \\
(\%)\end{array}$ & $\begin{array}{c}\text { Graus de } \\
\text { liberdade } \\
\text { totais }\end{array}$ \\
\hline 1 & 1,255 & 2,098 & 40,18 & 1891 \\
\hline 2 & 1,540 & 2,098 & 26,60 & 2047 \\
\hline 3 & 1,747 & 2,098 & 16,73 & 2203 \\
\hline 4 & 1,881 & 2,098 & 10,34 & 2359 \\
\hline 5 & 2,073 & 2,098 & 1,19 & 2515 \\
\hline 6 & 2,217 & 2,098 & 5,67 & 2671 \\
\hline
\end{tabular}

Analisando os dados das tabelas verifica-se que os enriquecimentos hierárquicos e trigonométricos com uma dimensão da base enriquecedora igual a cinco fornecem os melhores resultados. Já o enriquecimento com a base não-hierárquica fornece resultados piores que os demais. 
Comparando os resultados da Tabela $6.6 \mathrm{com}$ as demais, verifica-se uma grande queda do erro relativo, comprovando a melhora na aproximação da solução ao utilizar o enriquecimento.

Observa-se que ao utilizar uma dimensão da base enriquecedora igual a seis o erro relativo volta a subir. Isso pode ser justificado pelo fato que o não atendimento da condição (3.45) causa uma instabilidade na aproximação da solução.

\subsubsection{2- Carregamento linear aplicado na face da fissura}

O segundo subproblema local é ilustrado na figura abaixo:

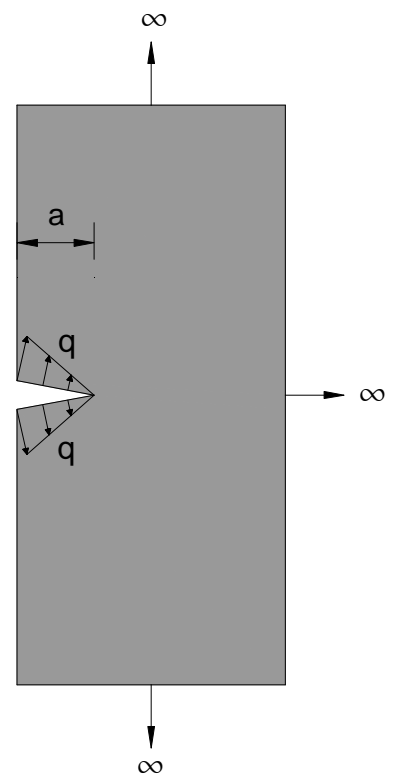

Figura 6.14 - Segundo subproblema local

O problema foi modelado explorando-se a simetria de acordo com a Figura 6.15. 


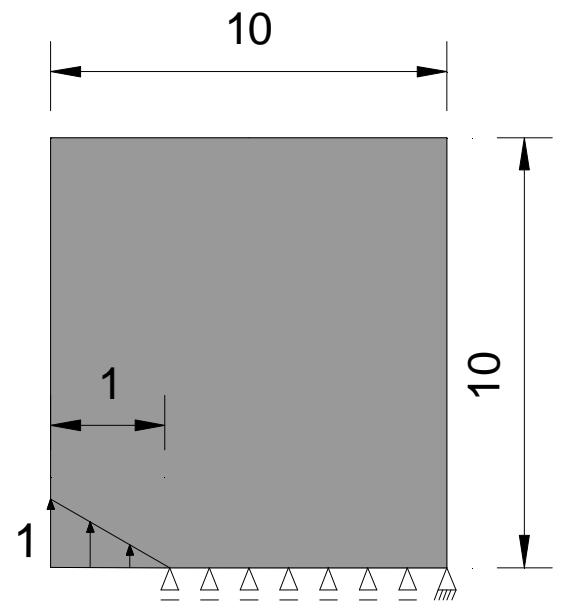

Figura 6.15 - Modelo do segundo subproblema local

A curva $F I T_{i}$ x $r$ para o caso em que é utilizado o enriquecimento trigonométrico com uma dimensão da base enriquecedora igual a quatro é apresentada na Figura 6.16:

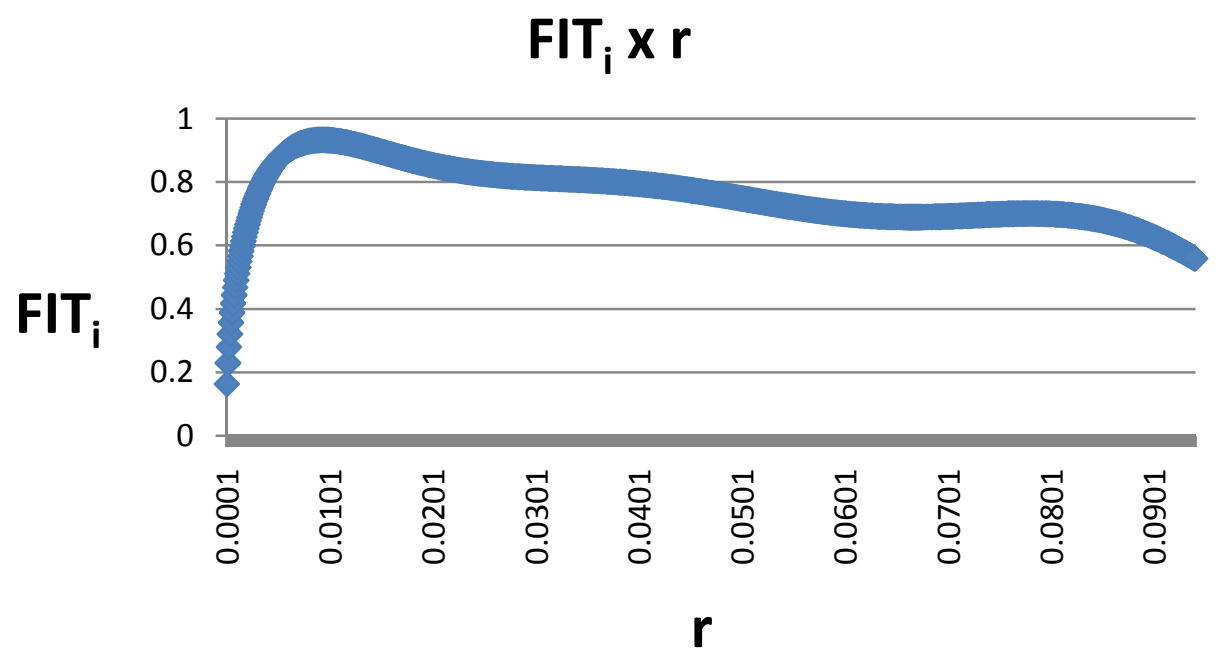

Figura 6.16 - Curva de FIT $_{i}$ x r para o segundo subproblema local

Assim como no subproblema anterior, a extração do FIT foi obtido calculando uma média utilizando o processo dos mínimos quadrados no trecho entre $r=0,01$ e $r=0,08$. Ao todo, foram coletados valores de $\mathrm{FIT}_{\mathrm{i}}$ em 700 pontos, sendo a distância entre eles igual a 0,001. O 
valor do FIT, bem como a comparação com o valor analítico é dado pelas Tabela 6.10 a Tabela 6.13:

Tabela 6.10 - Resultados do FIT sem enriquecimento

\begin{tabular}{c|c|c|c|c}
\hline \multicolumn{5}{c}{ SEM ENRIQUECIMENTO } \\
\hline $\begin{array}{c}\text { Dimensão da } \\
\text { base } \\
\text { enriquecedora }\end{array}$ & FIT final & $\begin{array}{c}\text { FIT } \\
\text { referência }\end{array}$ & $\begin{array}{c}\text { Erro relativo } \\
(\%)\end{array}$ & $\begin{array}{c}\text { Graus de } \\
\text { liberdade } \\
\text { totais }\end{array}$ \\
\hline 0 & 0,315 & 0,856 & 63,20 & 1735 \\
\hline
\end{tabular}

Tabela 6.11 - Resultados do FIT com enriquecimento hierárquico

\begin{tabular}{c|c|c|c|c}
\hline \multicolumn{5}{c}{ ENRIQUECIMENTO HIERÁRQUICO } \\
\hline $\begin{array}{c}\text { Dimensão da } \\
\text { base } \\
\text { enriquecedora }\end{array}$ & FIT final & $\begin{array}{c}\text { FIT } \\
\text { referência }\end{array}$ & $\begin{array}{c}\text { Erro } \\
\text { relativo } \\
(\%)\end{array}$ & $\begin{array}{c}\text { Graus de } \\
\text { liberdade } \\
\text { totais }\end{array}$ \\
\hline 1 & 0,594 & 0,856 & 30,61 & 1891 \\
\hline 2 & 0,752 & 0,856 & 12,15 & 2047 \\
\hline 3 & 0,814 & 0,856 & 4,91 & 2203 \\
\hline 4 & 0,869 & 0,856 & 1,52 & 2359 \\
\hline 5 & 0,938 & 0,856 & 9,58 & 2515 \\
\hline 6 & 0,991 & 0,856 & 15,77 & 2671 \\
\hline
\end{tabular}

Tabela 6.12 - Resultados do FIT com enriquecimento não-hierárquico

\begin{tabular}{c|c|c|c|c}
\hline \multicolumn{5}{c}{ ENRIQUECIMENTO NÃO-HIERÁRQUICO } \\
\hline $\begin{array}{c}\text { Dimensão da } \\
\text { base } \\
\text { enriquecedora }\end{array}$ & FIT final & $\begin{array}{c}\text { FIT } \\
\text { referência }\end{array}$ & $\begin{array}{c}\text { Erro } \\
\text { relativo } \\
(\%)\end{array}$ & $\begin{array}{c}\text { Graus de } \\
\text { liberdade } \\
\text { totais }\end{array}$ \\
\hline 1 & 0,510 & 0,856 & 40,42 & 1891 \\
\hline 2 & 0,704 & 0,856 & 17,76 & 2047 \\
\hline 3 & 0,825 & 0,856 & 3,62 & 2203 \\
\hline 4 & 0,834 & 0,856 & 2,57 & 2359 \\
\hline 5 & 0,918 & 0,856 & 7,24 & 2515 \\
\hline 6 & 0,982 & 0,856 & 14,72 & 2671 \\
\hline
\end{tabular}


Tabela 6.13 - Resultados do FIT com enriquecimento trigonométrico

\begin{tabular}{c|c|c|c|c}
\hline \multicolumn{5}{c}{ ENRIQUECIMENTO TRIGONOMÉTRICO } \\
\hline $\begin{array}{c}\text { Dimensão da } \\
\text { base } \\
\text { enriquecedora }\end{array}$ & FIT final & $\begin{array}{c}\text { FIT } \\
\text { referência }\end{array}$ & $\begin{array}{c}\text { Erro relativo } \\
(\%)\end{array}$ & $\begin{array}{c}\text { Graus de } \\
\text { liberdade } \\
\text { totais }\end{array}$ \\
\hline 1 & 0,587 & 0,856 & 31,43 & 1891 \\
\hline 2 & 0,710 & 0,856 & 17,06 & 2047 \\
\hline 3 & 0,792 & 0,856 & 7,48 & 2203 \\
\hline 4 & 0,849 & 0,856 & 0,82 & 2359 \\
\hline 5 & 0,924 & 0,856 & 7,91 & 2515 \\
\hline 6 & 0,987 & 0,856 & 15,30 & 2671 \\
\hline
\end{tabular}

Analisando os dados das tabelas verifica-se que os enriquecimentos hierárquicos e trigonométricos com uma dimensão da base enriquecedora igual a quatro fornecem os melhores resultados. Já o enriquecimento com a base não-hierárquica fornece resultados piores que os demais.

Comparando os resultados da Tabela 6.10 com as demais tabelas, verifica-se uma grande queda do erro relativo, comprovando a melhora na aproximação da solução ao utilizar o enriquecimento.

Observa-se que ao utilizar uma dimensão da base enriquecedora maior que quatro o erro relativo volta a subir. Mais uma vez, isso pode ser justificado pelo fato que o não atendimento do teste do "mosaico" está causando uma instabilidade na aproximação da solução.

\subsubsection{3- Carregamento quadrático aplicado na face da fissura}

O terceiro subproblema local está mostrado na Figura 6.17: 


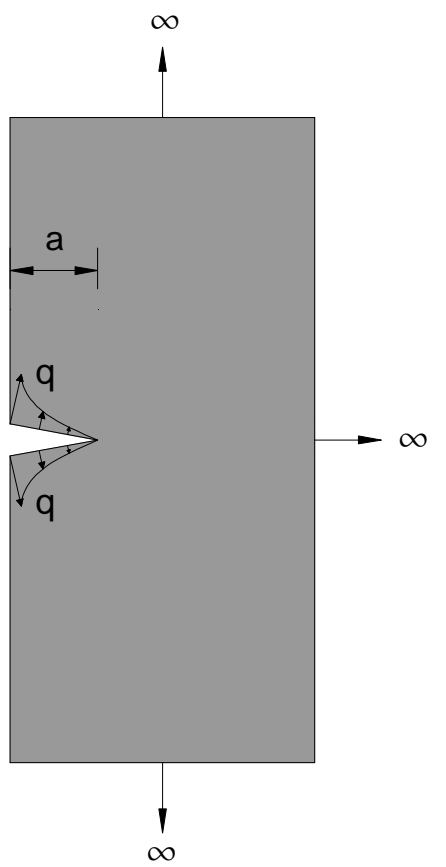

Figura 6.17 - Terceiro subproblema local

Novamente, a simetria foi explorada conforme representado na Figura 6.18.

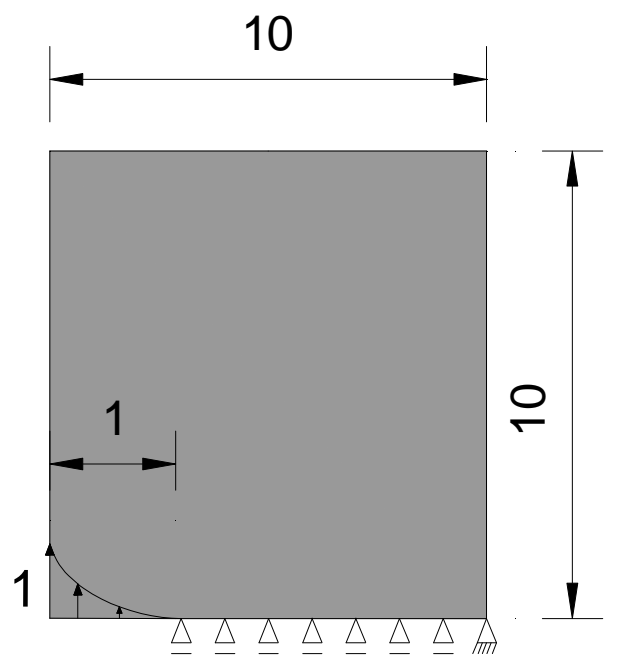

Figura 6.18 - Modelo do terceiro subproblema local

A curva $F I T_{i}$ x $r$ obtida para o caso em que é utilizado o enriquecimento hierárquico com uma dimensão da base enriquecedora igual a quatro está apresentada na Figura 6.19: 


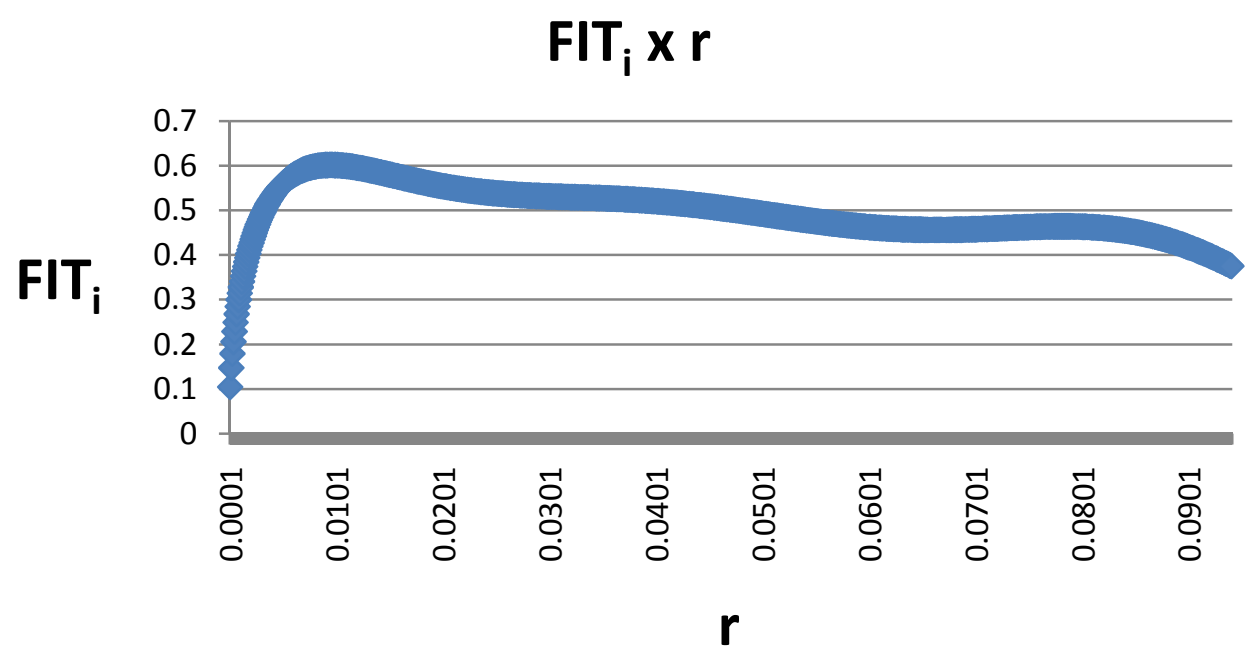

Figura 6.19 - Curva de FIT $_{\mathbf{i}}$ x r para o terceiro subproblema local

Assim como nos subproblemas anteriores, a extração do FIT foi obtida calculando uma média utilizando o processo dos mínimos quadrados no trecho entre $\mathrm{r}=0,01$ e $\mathrm{r}=0,08$. Ao todo, foram coletados valores de FIT $_{i}$ em 700 pontos, sendo a distância entre eles igual a 0,001. O valor do FIT, bem como a comparação com o valor analítico é dado pelas Tabela 6.14 a Tabela 6.17:

Tabela 6.14 - Resultados do FIT sem enriquecimento

\begin{tabular}{c|c|c|c|c}
\hline \multicolumn{5}{c}{ SEM ENRIQUECIMENTO } \\
\hline $\begin{array}{c}\text { Dimensão da } \\
\text { base } \\
\text { enriquecedora }\end{array}$ & FIT final & $\begin{array}{c}\text { FIT } \\
\text { referência }\end{array}$ & $\begin{array}{c}\text { Erro } \\
\text { relativo } \\
(\%)\end{array}$ & $\begin{array}{c}\text { Graus de } \\
\text { liberdade } \\
\text { totais }\end{array}$ \\
\hline 0 & 0,206 & 0,556 & 62,95 & 1735 \\
\hline
\end{tabular}


Tabela 6.15 - Resultados do FIT com enriquecimento hierárquico

\begin{tabular}{c|c|c|c|c}
\hline \multicolumn{5}{c}{ ENRIQUECIMENTO HIERÁRQUICO } \\
\hline $\begin{array}{c}\text { Dimensão da } \\
\text { base } \\
\text { enriquecedora }\end{array}$ & FIT final & $\begin{array}{c}\text { FIT } \\
\text { referência }\end{array}$ & $\begin{array}{c}\text { Erro } \\
\text { relativo } \\
(\%)\end{array}$ & $\begin{array}{c}\text { Graus de } \\
\text { liberdade } \\
\text { totais }\end{array}$ \\
\hline 1 & 0,386 & 0,556 & 30,58 & 1891 \\
\hline 2 & 0,488 & 0,556 & 12,23 & 2047 \\
\hline 3 & 0,529 & 0,556 & 4,86 & 2203 \\
\hline 4 & 0,562 & 0,556 & 1,08 & 2359 \\
\hline 5 & 0,606 & 0,556 & 8,99 & 2515 \\
\hline 6 & 0,640 & 0,556 & 15,11 & 2671 \\
\hline
\end{tabular}

Tabela 6.16 - Resultados do FIT com enriquecimento não-hierárquico

\begin{tabular}{c|c|c|c|c}
\hline \multicolumn{5}{c}{ ENRIQUECIMENTO NÃO-HIERÁRQUICO } \\
\hline $\begin{array}{c}\text { Dimensão da } \\
\text { base } \\
\text { enriquecedora }\end{array}$ & FIT final & $\begin{array}{c}\text { FIT } \\
\text { referência }\end{array}$ & $\begin{array}{c}\text { Erro } \\
\text { relativo } \\
(\%)\end{array}$ & $\begin{array}{c}\text { Graus de } \\
\text { liberdade } \\
\text { totais }\end{array}$ \\
\hline 1 & 0,330 & 0,556 & 40,65 & 1891 \\
\hline 2 & 0,457 & 0,556 & 17,81 & 2047 \\
\hline 3 & 0,535 & 0,556 & 3,78 & 2203 \\
\hline 4 & 0,540 & 0,556 & 2,88 & 2359 \\
\hline 5 & 0,593 & 0,556 & 6,65 & 2515 \\
\hline 6 & 0,633 & 0,556 & 13,85 & 2671 \\
\hline
\end{tabular}

Tabela 6.17 - Resultados do FIT com enriquecimento trigonométrico

\begin{tabular}{c|c|c|c|c}
\hline \multicolumn{5}{c}{ ENRIQUECIMENTO TRIGONOMÉTRICO } \\
\hline $\begin{array}{c}\text { Dimensão da } \\
\text { base } \\
\text { enriquecedora }\end{array}$ & FIT final & $\begin{array}{c}\text { FIT } \\
\text { referência }\end{array}$ & $\begin{array}{c}\text { Erro } \\
\text { relativo } \\
(\%)\end{array}$ & $\begin{array}{c}\text { Graus de } \\
\text { liberdade } \\
\text { totais }\end{array}$ \\
\hline 1 & 0,382 & 0,556 & 31,29 & 1891 \\
\hline 2 & 0,460 & 0,556 & 17,27 & 2047 \\
\hline 3 & 0,513 & 0,556 & 7,73 & 2203 \\
\hline 4 & 0,549 & 0,556 & 1,26 & 2359 \\
\hline 5 & 0,597 & 0,556 & 7,41 & 2515 \\
\hline 6 & 0,638 & 0,556 & 14,75 & 2671 \\
\hline
\end{tabular}

Analisando os dados das tabelas, verifica-se que os enriquecimentos hierárquicos e trigonométricos com uma dimensão da base enriquecedora igual a quatro fornecem os 
melhores resultados. Já o enriquecimento com a base não-hierárquica fornece resultados piores que os demais.

Comparando os resultados da Tabela 6.14 com as demais tabelas, verifica-se uma grande queda do erro relativo, comprovando a melhora na aproximação da solução ao utilizar o enriquecimento.

Observa-se, novamente, que ao utilizar uma dimensão da base enriquecedora maior que quatro, o erro relativo volta a subir. Isso pode ser justificado pelo fato que o não atendimento do teste do "mosaico" está causando uma instabilidade na aproximação da solução.

\subsubsection{4- Conclusões gerais da análise utilizando a formulações híbrido-Trefftz}

Ao analisar os três casos, observa-se que os melhores resultados são obtidos utilizando as bases enriquecedoras hierárquicas e trigonométricas até a dimensão igual a quatro. Os resultados obtidos com a base não-hierárquica não foram tão efetivos, talvez porque as funções dessa base não apresentam uma distribuição simétrica (Figura 3.4).

\subsection{2 - Análise utilizando o MEFG}

Para a modelagem dos três casos não foi utilizada a simetria horizontal do problema. Assim como na análise com a formulação híbrido-Trefftz, considerou-se a dimensão infinita da chapa como uma dimensão igual ao da chapa do $3^{\circ}$ problema (que será visto adiante), pois nele serão utilizados esses mesmos subproblemas $P_{L}^{(k)}$.

A rede de elementos finitos utilizada nos três casos pode ser visualizada na Figura 6.20. Ela contém um total de 717 nós e 656 elementos. Todos os nós da rede foram enriquecidos em ambas as direções ( $\mathrm{x}$ e $\mathrm{y}$ ), exceto aqueles vinculados. As funções enriquecedoras empregadas são: $\mathrm{x}, \mathrm{x}^{2}, \mathrm{x}^{3}, \mathrm{x}^{4}, \mathrm{x}^{5}, F x$ na direção $\mathrm{x}$ e y, $\mathrm{y}^{2}, \mathrm{y}^{3}, \mathrm{y}^{4}, \mathrm{y}^{5}, F y$ na direção y. Contando com o enriquecimento, totalizam 9870 graus de liberdade.

O detalhe do interior do contorno gama pode ser visto na Figura 6.21. 


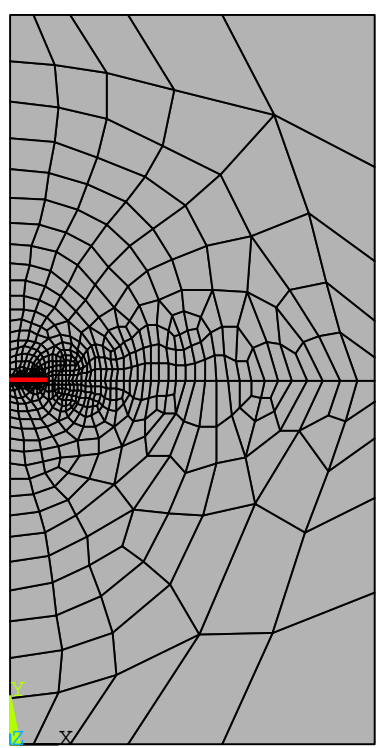

Figura 6.20 - Rede de elementos finitos do segundo problema utilizando o MEFG

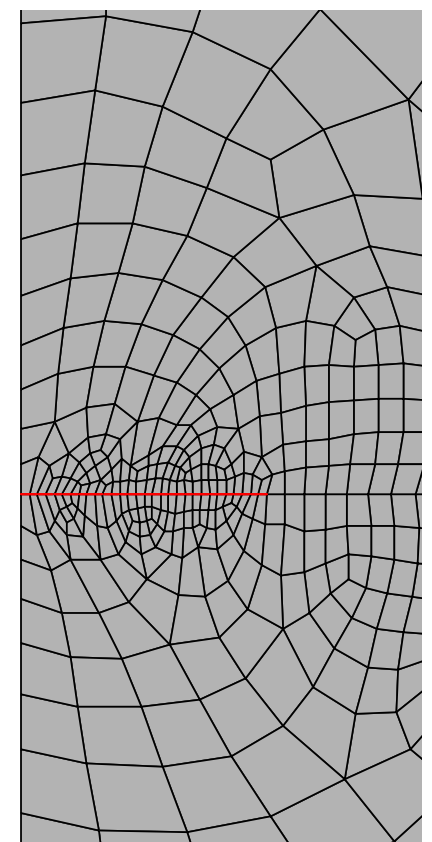

Figura 6.21 - Detalhe da região interna ao contorno gama 


\subsubsection{1 - Carregamento uniforme aplicado na face da fissura}

O primeiro subproblema local é dado pela Figura 6.11 e seu modelo é representado pela Figura 6.22.

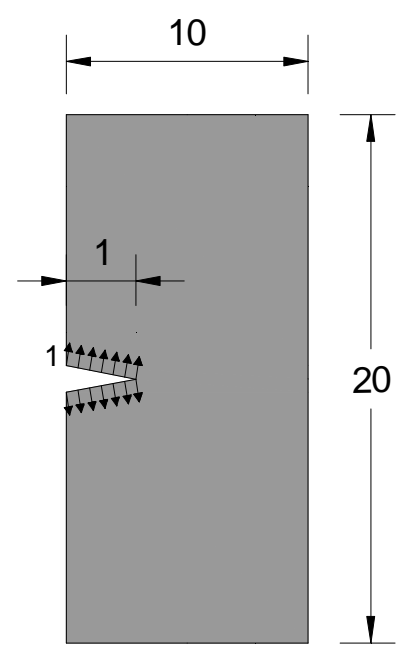

Figura 6.22 - Modelo para o primeiro subproblema local

A extração do FIT foi obtida utilizando a técnica de correlação dos deslocamentos. Os nós utilizados para a extração do FIT ficam em um trecho correspondente a $10 \%$ do comprimento da fissura. O resultado obtido pode ser visualizado na Tabela 6.18.

Tabela 6.18 - Resultados do FIT com MEFG

\begin{tabular}{c|c|c|c}
\hline FIT obtido & FIT referência & $\begin{array}{c}\text { Erro relativo } \\
(\%)\end{array}$ & $\begin{array}{c}\text { Graus de } \\
\text { liberdad } \\
\text { e totais }\end{array}$ \\
\hline 2,083 & 2,098 & 0,71 & 9870 \\
\hline
\end{tabular}

\subsubsection{2 - Carregamento linear aplicado na face da fissura}

O segundo subproblema local é o mesmo dado pela Figura 6.14 e reproduzido pela Figura 6.23 . 


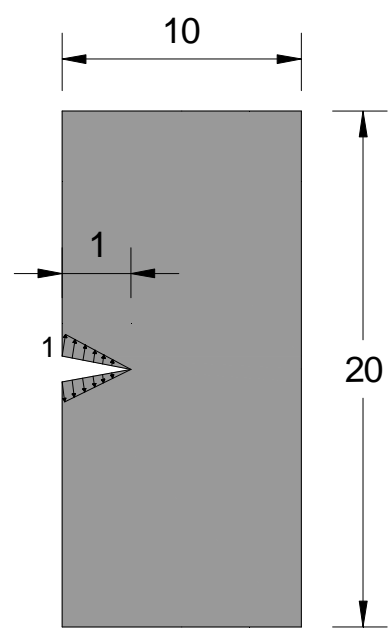

Figura 6.23 - Modelo para o segundo subproblema local

Assim como nos subproblemas anteriores, a extração do FIT foi obtida utilizando a técnica de correlação dos deslocamentos. Os nós utilizados para a extração do FIT ficam em um trecho correspondente a $10 \%$ do comprimento da fissura. O resultado obtido pode ser visualizado na Tabela 6.19 .

Tabela 6.19 - Resultados do FIT com MEFG

\begin{tabular}{c|c|c|c}
\hline FIT obtido & FIT referência & $\begin{array}{c}\text { Erro relativo } \\
(\%)\end{array}$ & $\begin{array}{c}\text { Graus de } \\
\text { liberdad } \\
\text { e totais }\end{array}$ \\
\hline 0,893 & 0,856 & 5,37 & 9870 \\
\hline
\end{tabular}

\subsubsection{3 - Carregamento quadrático aplicado na face da fissura}

O terceiro subproblema local é dado pela Figura 6.17. Seu modelo é representado pela Figura 6.24. 


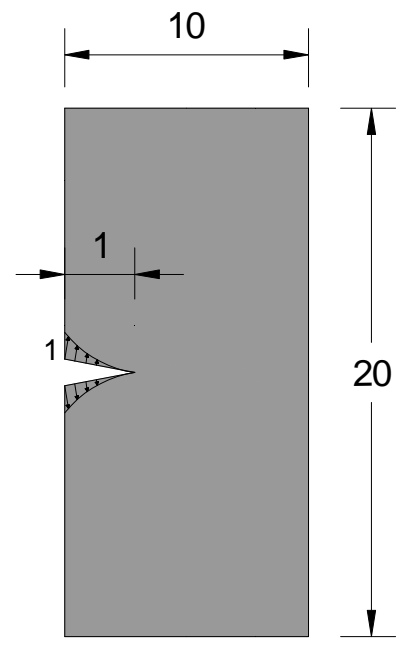

Figura 6.24 - Modelo para o terceiro subproblema local

Assim como nos subproblemas anteriores, a extração do FIT foi obtida utilizando a técnica de correlação dos deslocamentos. Os nós utilizados para a extração do FIT ficam a uma distância de $10 \%$ do comprimento da fissura.

Tabela 6.20 - Resultados do FIT com MEFG

\begin{tabular}{c|c|c|c}
\hline FIT obtido & FIT referência & $\begin{array}{c}\text { Erro relativo } \\
(\%)\end{array}$ & $\begin{array}{c}\text { Graus de } \\
\text { liberdad } \\
\text { e totais }\end{array}$ \\
\hline 0,569 & 0,556 & 6,35 & 9870 \\
\hline
\end{tabular}

\subsubsection{4- Conclusões gerais da análise utilizando o MEFG}

Observa-se que o valor do erro relativo indicado nas Tabela 6.19 e Tabela 6.20 está alto em relação a um critério em que $1 \%$ seria o erro desejável. Porém ao aplicar a sobreposição de todos os subproblemas propostos pelo Método da Partição, o erro relativo do FIT final das fissuras tende a diminuir. Isso será visto no terceiro problema. 


\section{4- 3o Problema}

O terceiro problema trata de uma chapa contendo duas fissuras horizontais, iguais e simétricas. A chapa está submetida a um carregamento de tração em ambas as bordas horizontais (Figura 6.25).

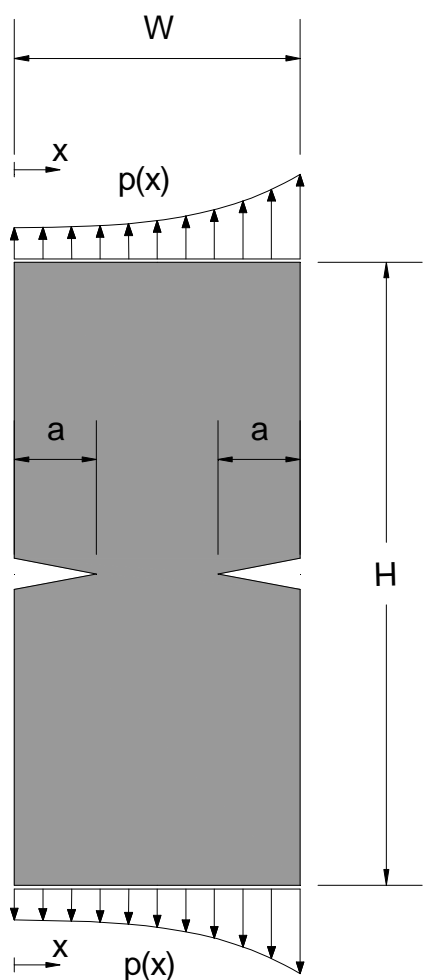

Figura 6.25 - Chapa com duas fissuras e carregamento não - uniforme

No dimensionamento, adotou-se a largura $W$ da chapa igual a 10, o comprimento $H$ da chapa igual a 20 e o comprimento "a" das fissuras igual a 1 . O carregamento $p(x)$ é descrito pela expressão:

$$
p(x)=1+\left(\frac{x}{10}\right)^{2}
$$




\subsection{1- Análise utilizando a formulação híbrido-Trefftz}

Para a modelagem foi explorada a simetria horizontal do problema nas redes dos subproblemas $\mathrm{P}_{\mathrm{G}}^{(0)}$ e $\mathrm{P}_{\mathrm{G}}^{(\mathrm{k})}$ (redes iguais) e na do subproblema $P_{L}^{(k)}$.

$\mathrm{O}$ modelo para os subproblemas $\mathrm{P}_{\mathrm{G}}^{(0)}$ e $\mathrm{P}_{\mathrm{G}}^{(\mathrm{k})}$ está representado pela Figura 6.26. Os mesmos subproblemas locais apresentados no segundo problema foram adotados para esse problema, com um total de três termos de aproximação. Seus modelos são aqueles representados nas figuras Figura 6.12, Figura 6.15 e Figura 6.18.

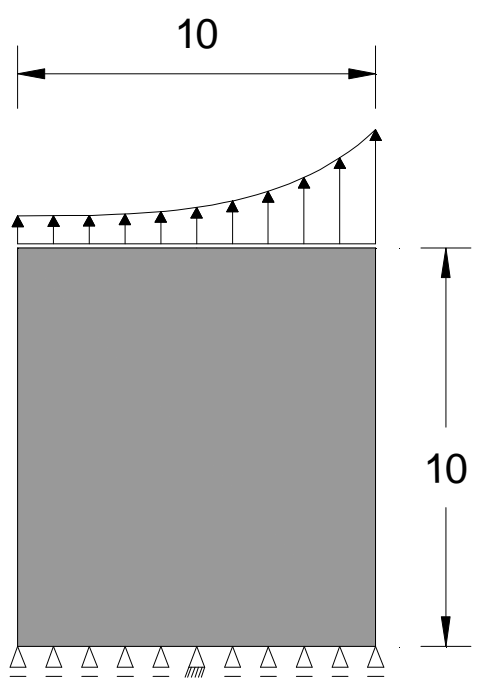

Figura 6.26 - Modelo para os subproblemas $P_{G}^{(0)}$ e $P_{G}^{(k)}$

A rede utilizada nos subproblemas $P_{G}^{(0)}$ e $P_{G}^{(k)}$ está representada na Figura 6.27, sendo composta por 391 nós e 352 elementos. Como nesses problemas não há presença de fissura, a análise foi feita pelo MEF clássico. 


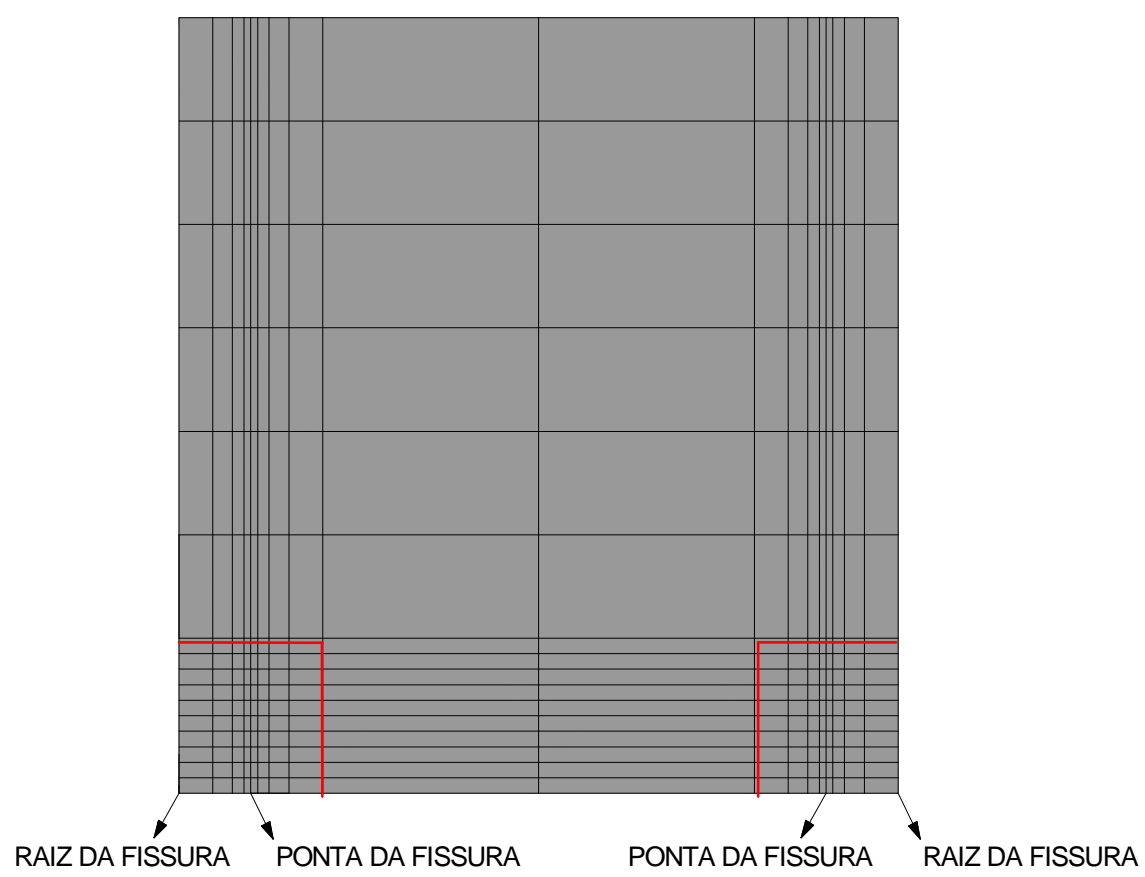

Figura 6.27 - Rede utilizada nos subproblemas $P_{G}^{(0)}$ e $P_{G}^{(k)}$

Na Figura 6.27 as linhas em vermelho representam o contorno gama. Vale lembrar que nesses dois subproblemas não há a presença de fissuras. Elas estão indicadas nessa figura apenas para ilustração.

Observa-se que a rede dos subproblemas $P_{G}^{(0)}$ e $P_{G}^{(k)}$ é mais refinada que a rede do subproblema $P_{L}^{(k)}$. Isso se deve ao fato que no subproblema $P_{L}^{(k)}$ apenas uma fissura é considerada para cada rede. Já nos subproblemas $P_{G}^{(0)}$ e $P_{G}^{(k)}$, deve-se ter linhas de nós que representem as duas fissuras e linhas de nós que representem o contorno gama.

Os resultados dos FIT finais obtidos com a sobreposição descrita no Método da Partição são apresentados nas Tabela 6.21 a Tabela 6.24. Inicialmente, apresenta-se na Tabela $6.21 \mathrm{o}$ resultado para a análise sem enriquecimento (base $\beta_{0}$ ). Em seguida são mostrados os resultados para os enriquecimentos utilizando as bases $\beta_{1}, \beta_{2}$ e $\beta_{3}$ variando a dimensão das bases enriquecedoras. Vale enfatizar que todos os trechos da rede foram enriquecidos, com exceção daqueles que estão vinculados. 
Tabela 6.21 - Resultados do $3^{\circ}$ problema sem enriquecimento

\begin{tabular}{c|c|c|c|c|c|c}
\hline \multicolumn{7}{c}{ SEM ENRIQUECIMENTO } \\
\hline $\begin{array}{c}\text { Dimensão da } \\
\text { base } \\
\text { enriquecedora }\end{array}$ & $\begin{array}{c}\text { FIT } \\
\text { final }\end{array}$ & $\begin{array}{c}\text { FIT } \\
\text { final }\end{array}$ & $\begin{array}{c}\text { FIT } \\
\text { fiss 1 } \\
\text { fiss 2 } \\
\text { fiss 1 }\end{array}$ & $\begin{array}{c}\text { FIT } \\
\text { referência } \\
\text { fiss 2 }\end{array}$ & $\begin{array}{c}\text { Erro } \\
\text { relativo } \\
\text { fiss 1 }(\%)\end{array}$ & $\begin{array}{c}\text { Erro } \\
\text { relativo } \\
\text { fiss 2 } \\
(\%)\end{array}$ \\
\hline 0 & 0,507 & 1,108 & 1,633 & 3,642 & 68,95 & 69,58 \\
\hline
\end{tabular}

Tabela 6.22 - Resultados do $3^{\circ}$ problema com enriquecimento hierárquico

\begin{tabular}{c|c|c|c|c|c|c}
\hline \multicolumn{7}{c}{ ENRIQUECIMENTO HIERÁRQUICO } \\
\hline $\begin{array}{c}\text { Dimensão da } \\
\text { base } \\
\begin{array}{c}\text { enriquecedor } \\
\text { a }\end{array}\end{array}$ & $\begin{array}{c}\text { FIT } \\
\text { final } \\
\text { fiss 1 }\end{array}$ & $\begin{array}{c}\text { FIT } \\
\text { final } \\
\text { fiss 2 }\end{array}$ & $\begin{array}{c}\text { FIT } \\
\text { referência } \\
\text { fiss 1 }\end{array}$ & $\begin{array}{c}\text { FIT } \\
\text { referência } \\
\text { fiss 2 }\end{array}$ & $\begin{array}{c}\text { Erro } \\
\text { relativo } \\
\text { fiss 1 } \\
(\%)\end{array}$ & $\begin{array}{c}\text { Erro } \\
\text { relativo } \\
\text { fiss 2 } \\
(\%)\end{array}$ \\
\hline 1 & 1,004 & 2,221 & 1,633 & 3,642 & 38,52 & 39,02 \\
\hline 2 & 1,279 & 2,848 & 1,633 & 3,642 & 21,68 & 21,80 \\
\hline 3 & 1,401 & 3,120 & 1,633 & 3,642 & 14,21 & 14,33 \\
\hline 4 & 1,501 & 3,343 & 1,633 & 3,642 & 8,08 & 8,21 \\
\hline 5 & 1,635 & 3,640 & 1,633 & 3,642 & 0,12 & 0,05 \\
\hline 6 & 1,732 & 3,856 & 1,633 & 3,642 & 6,06 & 5,88 \\
\hline
\end{tabular}

Tabela 6.23 - Resultados do $3^{\circ}$ problema com enriquecimento não-hierárquico

\section{ENRIQUECIMENTO NÃO-HIERÁRQUICO}

\begin{tabular}{c|c|c|c|c|c|c}
\hline $\begin{array}{c}\text { Dimensão da } \\
\text { base } \\
\text { enriquecedora }\end{array}$ & $\begin{array}{c}\text { FIT } \\
\text { final } \\
\text { fiss 1 }\end{array}$ & $\begin{array}{c}\text { FIT } \\
\text { final } \\
\text { fiss 2 }\end{array}$ & $\begin{array}{c}\text { FIT } \\
\text { referência } \\
\text { fiss 1 }\end{array}$ & $\begin{array}{c}\text { FIT } \\
\text { referência } \\
\text { fiss 2 }\end{array}$ & $\begin{array}{c}\text { Erro } \\
\text { relativo } \\
\text { fiss 1 } \\
(\%)\end{array}$ & $\begin{array}{c}\text { Erro } \\
\text { relativo } \\
\text { fiss 2 } \\
(\%)\end{array}$ \\
\hline 1 & 0,861 & 1,895 & 1,633 & 3,642 & 47,27 & 47,97 \\
\hline 2 & 1,180 & 2,632 & 1,633 & 3,642 & 27,74 & 27,73 \\
\hline 3 & 1,400 & 3,121 & 1,633 & 3,642 & 14,27 & 14,31 \\
\hline 4 & 1,437 & 3,200 & 1,633 & 3,642 & 12,00 & 12,14 \\
\hline 5 & 1,596 & 3,554 & 1,633 & 3,642 & 2,27 & 2,42 \\
\hline 6 & 1,714 & 3,817 & 1,633 & 3,642 & 4,96 & 4,81 \\
\hline
\end{tabular}


Tabela 6.24 - Resultados do $3^{\circ}$ problema com enriquecimento trigonométrico

\begin{tabular}{c|c|c|c|c|c|c}
\hline \multicolumn{7}{c}{ ENRIQUECIMENTO TRIGONOMÉTRICO } \\
\hline $\begin{array}{c}\text { Dimensão da } \\
\text { base } \\
\begin{array}{c}\text { enriquecedor } \\
\text { a }\end{array}\end{array}$ & $\begin{array}{c}\text { FIT } \\
\text { final } \\
\text { fiss 1 }\end{array}$ & $\begin{array}{c}\text { FIT } \\
\text { final } \\
\text { fiss 2 }\end{array}$ & $\begin{array}{c}\text { FIT } \\
\text { referência } \\
\text { fiss 1 }\end{array}$ & $\begin{array}{c}\text { FIT } \\
\text { referência } \\
\text { fiss 2 }\end{array}$ & $\begin{array}{c}\text { Erro } \\
\text { relativo } \\
\text { fiss 1 (\%) }\end{array}$ & $\begin{array}{c}\text { Erro } \\
\text { relativo } \\
\text { fiss 2 (\%) }\end{array}$ \\
\hline 1 & 0,988 & 2,183 & 1,633 & 3,642 & 39,50 & 40,06 \\
\hline 2 & 1,202 & 2,673 & 1,633 & 3,642 & 26,39 & 26,61 \\
\hline 3 & 1,361 & 3,029 & 1,633 & 3,642 & 16,66 & 16,83 \\
\hline 4 & 1,464 & 3,260 & 1,633 & 3,642 & 10,35 & 10,49 \\
\hline 5 & 1,613 & 3,591 & 1,633 & 3,642 & 1,22 & 1,40 \\
\hline 6 & 1,725 & 3,841 & 1,633 & 3,642 & 5,63 & 5,46 \\
\hline
\end{tabular}

Analisando os dados das tabelas acima verifica-se que os menores erros relativos para os FIT finais são atingidos quando se utiliza o enriquecimento hierárquico e o enriquecimento trigonométrico com uma dimensão da base enriquecedora igual a cinco. Já o enriquecimento com a base não-hierárquica fornece resultados piores que os demais.

Comparando os resultados da Tabela $6.21 \mathrm{com}$ as demais tabelas verifica-se uma grande queda do erro relativo, comprovando a melhora na aproximação da solução ao utilizar o enriquecimento.

Observa-se, novamente, que ao utilizar uma dimensão da base enriquecedora maior que cinco o erro relativo volta a subir. Isso pode ser justificado pelo fato que o não atendimento do teste do "mosaico" está causando uma instabilidade na aproximação da solução.

\subsection{2 - Análise utilizando o MEFG}

Para a modelagem com o MEFG não foi utilizada a simetria horizontal do problema. Assim como na análise com a formulação híbrido-Trefftz, os subproblemas $P_{L}^{(k)}$ são os mesmos utilizados no segundo problema. Assim, adotaram-se três termos de aproximação.

O modelo para os subproblemas $P_{G}^{(0)}$ e $P_{G}^{(k)}$ está representado pela Figura 6.28. Os modelos para os subproblemas $P_{L}^{(k)}$ são representados pelas Figura 6.22, Figura 6.23 e Figura 6.24 . 


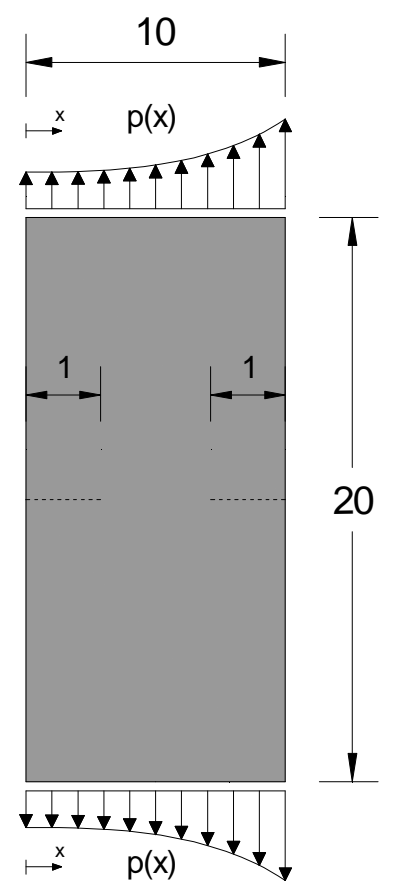

Figura 6.28 - Modelo para os subproblemas $P_{G}^{(0)}$ e $P_{G}^{(k)}$

A rede utilizada nos subproblemas $P_{G}^{(0)}$ e $P_{G}^{(k)}$ está representada na Figura 6.29. Ela é composta por 542 nós e 519 elementos. Como nesse problema não há presença de fissura, a análise foi feita pelo MEF clássico.

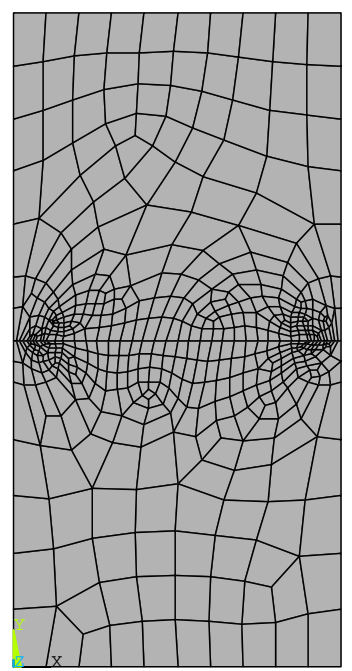

Figura 6.29 - Rede dos subproblemas $\mathrm{P}_{G}^{(0)}$ e $\mathrm{P}_{G}^{(k)}$ do $3^{\text {o }}$ problema 
Os resultados dos FIT finais obtidos são apresentados na Tabela 6.25.

Tabela 6.25 - Resultados do $3^{\circ}$ problema com o MEFG

\begin{tabular}{c|c|c|c|c|c}
\hline $\begin{array}{c}\text { FIT } \\
\text { final } \\
\text { fiss 1 }\end{array}$ & $\begin{array}{c}\text { FIT } \\
\text { final } \\
\text { fiss 2 }\end{array}$ & $\begin{array}{c}\text { FIT } \\
\text { referência } \\
\text { fiss 1 }\end{array}$ & $\begin{array}{c}\text { FIT } \\
\text { referência } \\
\text { fiss 2 }\end{array}$ & $\begin{array}{c}\text { Erro } \\
\text { relativo } \\
\text { fiss 1 } \\
(\%)\end{array}$ & $\begin{array}{c}\text { Erro } \\
\text { relativo } \\
\text { fiss 2 } \\
(\%)\end{array}$ \\
\hline 1,630 & 3,585 & 1,633 & 3,642 & 0,18 & 1,57 \\
\hline
\end{tabular}

Observa-se da Tabela 6.25 que o erro relativo do FIT referente à segunda fissura foi maior que a primeira. Isso se deve ao fato de que a concentração de tensão na segunda fissura é mais intensa devido à distribuição não-uniforme do carregamento nas bordas horizontais da chapa.

\section{5- 4음oblema}

O quarto problema trata de uma chapa contendo duas fissuras horizontais, iguais e simétricas, cuja origem está na borda de dois semi-círculos que idealizam furos de rebites. A chapa está submetida a um carregamento uniformemente distribuído em ambas as bordas horizontais (Figura 6.30). 


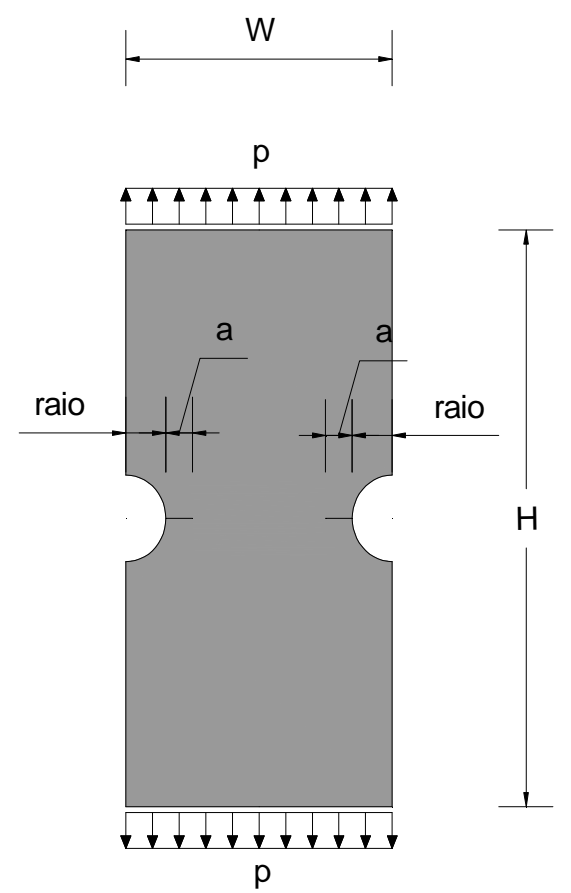

Figura $6.30-4^{\circ}$ problema

Em relação aos dados geométricos, adotou-se a largura $W$ da chapa igual a 10 , o comprimento $H$ igual a 20 e o comprimento "a" das fissuras igual a 1 . O carregamento $p$ vale 1.

Os valores de referência finais para os FIT de cada fissura foram obtidos no trabalho de Alves (2010). Para modelagem dos subproblemas $P_{L}^{(k)}$ utilizaram-se elementos finitos quadrilaterais de oito nós (deslocamentos em duas direções por nó), aproveitando a simetria horizontal do problema. Ao total foram 41742 nós e 13813 elementos (83484 graus de liberdades totais). A análise foi realizada utilizando a formulação do MEF clássico. Naquele mesmo trabalho foi observado que a presença dos furos causa uma perturbação adicional no campo de tensões do subproblema $P_{G}^{(0)}$. Assim, a base aproximativa polinomial exige muitos termos para capturar a solução exata dos subproblemas. Babuška e Andersson (2005) definem o espaço das funções que atendem a este tipo de problema:

$$
L_{\alpha, \beta}(\text { fiss })=\left\{Q \mid \int_{\text {fiss }} Q^{2}(\xi) \cdot(1-\xi)^{\alpha}(1+\xi)^{\beta} \partial \xi=\|Q\|_{L_{\alpha \beta(f i s s)}}^{2}<\infty,-1<\alpha, \beta\right\} \text { (6.4) }
$$


portanto, foram adotados valores de $\alpha$ e $\beta$ iguais a 0 e $-1 / 2$, respectivamente, obtendo as funções de aproximação para esse problema:

$$
\underline{Q}_{j}\left(\xi_{i} / a_{i}\right)=\frac{1}{\sqrt{1+\xi_{i}}}\left(\frac{\xi_{i}}{a_{i}}\right)^{j-1}
$$

\subsection{1 - Análise utilizando a formulação híbrido-Trefftz}

Para a modelagem foi explorada a simetria horizontal do problema nas redes dos subproblemas $P_{G}^{(0)}$ e $P_{G}^{(k)}$, bem como na do subproblema $P_{L}^{(k)}$. Adotou-se um total de cinco termos de aproximação dos campos de tensão nas faces da fissura.

O modelo para os subproblemas $P_{G}^{(0)}$ e $P_{G}^{(k)}$ é representado pela Figura 6.31 e o modelo para o subproblema $P_{L}^{(k)}$ é representado pela Figura 6.32

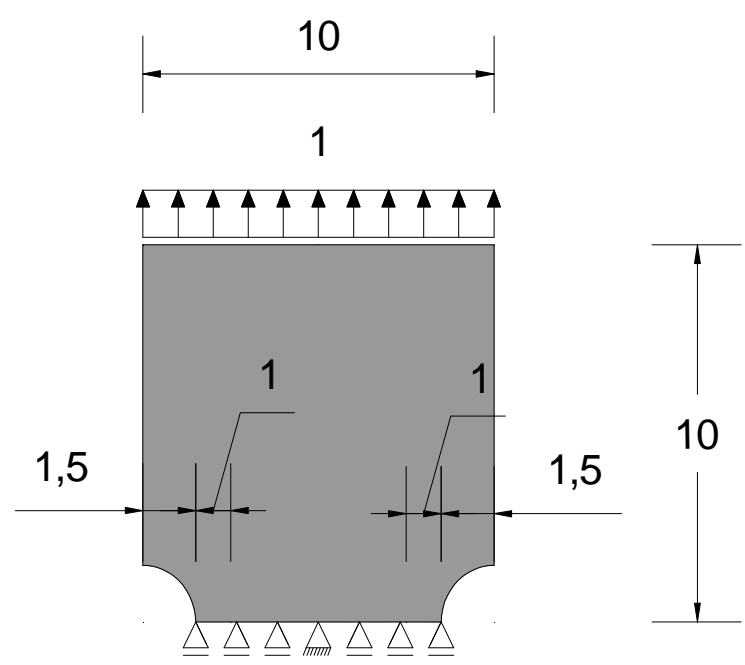

Figura 6.31 - Modelo dos subproblemas $P_{G}^{(0)}$ e $P_{G}^{(k)}$ do $4^{0}$ problema 


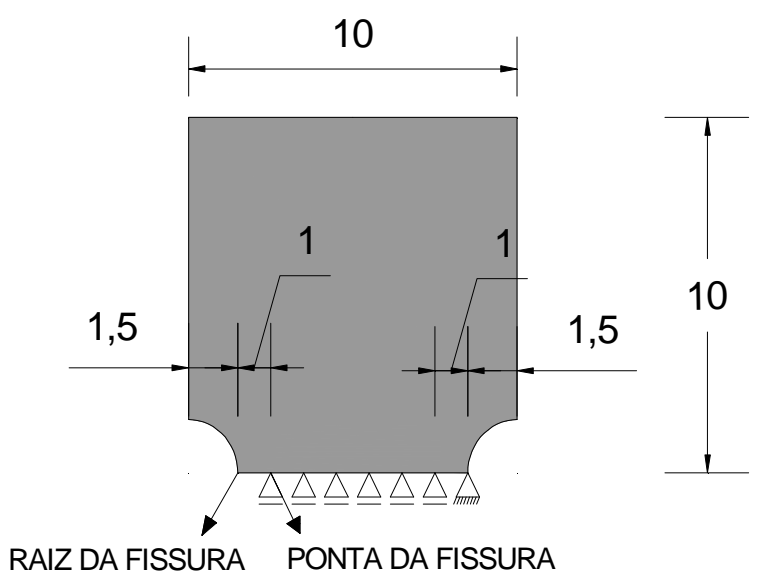

Figura 6.32 - Modelo do subproblema $P_{L}^{(k)}$ do $4^{0}$ problema

A rede utilizada nos subproblemas $P_{G}^{(0)}$ e $P_{G}^{(k)}$ está representada na Figura 6.33, sendo composta por 217 nós e 182 elementos. Como nesse problema não há presença de fissura, a análise foi feita pelo MEF clássico.

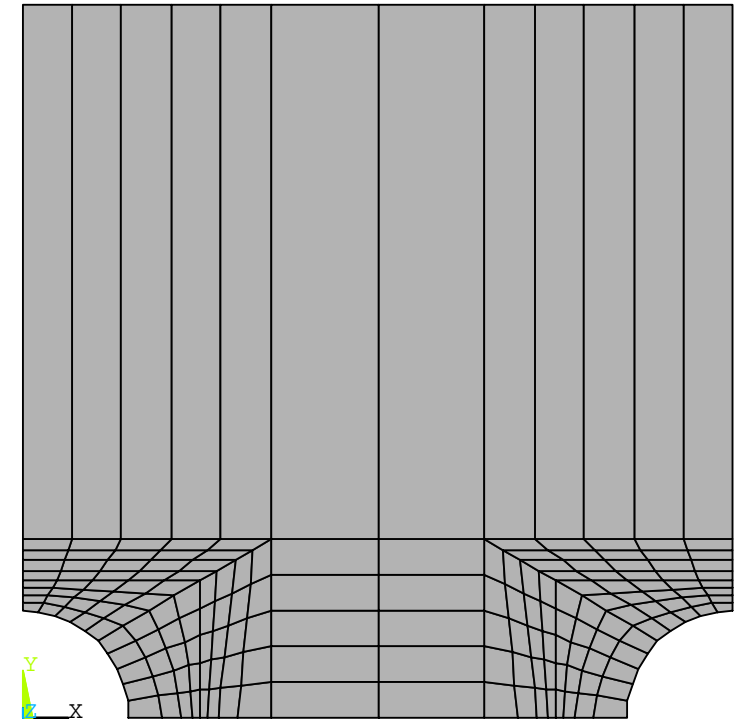

Figura 6.33 - Rede dos subproblemas $P_{G}^{(0)}$ e $P_{G}^{(k)}$ do $4^{0}$ problema

A rede do subproblema $P_{L}^{(k)}$ está representada na Figura 6.34 e a ampliação da região próximo à fissura é mostrada na Figura 6.35. A rede é composta por 128 nós e 104 elementos. 


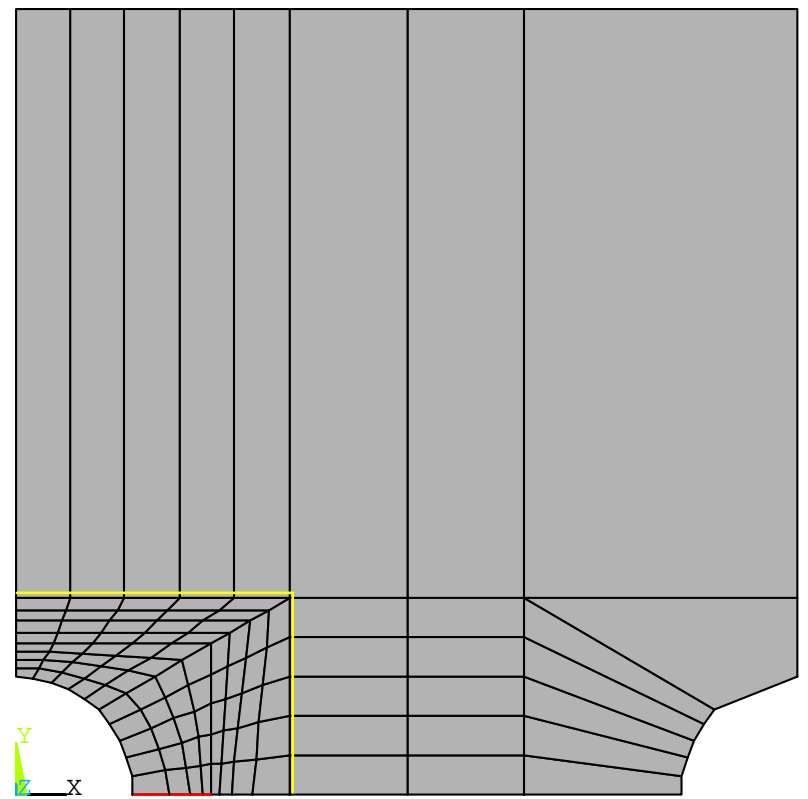

Figura 6.34 - Rede do subproblema $P_{L}^{(k)}$ do $4^{0}$ problema

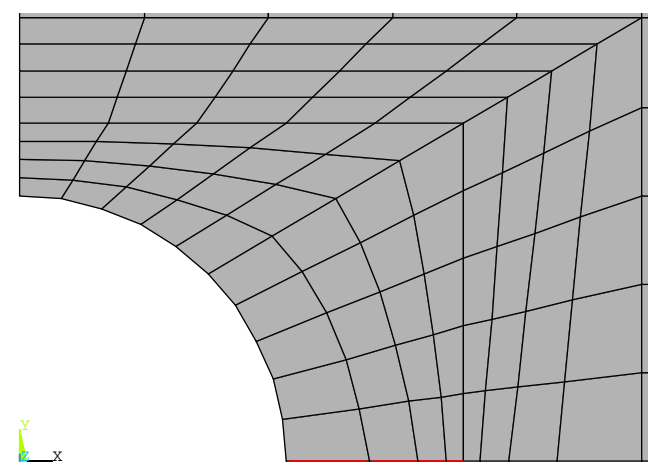

Figura 6.35 - Detalhe do contorno gama da rede do subproblema $P_{L}^{(k)}$ do $4^{0}$ problema

Na Figura 6.35 a linha em vermelho representa a fissura e as linhas amarelas representam o contorno gama.

O fato da rede dos subproblemas $P_{G}^{(0)}$ e $P_{G}^{(k)}$ ser mais refinada que a do subproblema $P_{L}^{(k)}$ segue a mesma idéia apresentada no $3^{\circ}$ problema.

Os resultados obtidos são reunidos nas Tabela 6.26 a Tabela 6.29. Inicialmente, na Tabela 6.26 apresenta-se o resultado para a análise sem enriquecimento (base $\beta_{0}$ ). Em seguida são mostrados os resultados para os enriquecimentos utilizando as bases $\beta_{1}, \beta_{2}$ e $\beta_{3}$ variando a dimensão das bases enriquecedoras. Vale enfatizar que todos os trechos da rede foram 
enriquecidos, com exceção daqueles que estão vinculados. Como as fissuras são iguais, simétricas e submetidas a um carregamento também simétrico, o valor do FIT final é o mesmo para ambas as fissuras.

Tabela 6.26 - Resultados do $4^{\circ}$ problema sem enriquecimento

\begin{tabular}{c|c|c|c|c}
\hline \multicolumn{5}{|c|}{ SEM ENRIQUECIMENTO } \\
\hline $\begin{array}{c}\text { Dimensão da } \\
\text { base } \\
\text { enriquecedora }\end{array}$ & FIT final & $\begin{array}{c}\text { FIT } \\
\text { referência }\end{array}$ & $\begin{array}{c}\text { Erro } \\
\text { relativo }(\%)\end{array}$ & $\begin{array}{c}\text { Graus de } \\
\text { liberdade } \\
\text { totais do } \\
P_{L}^{(k)}\end{array}$ \\
\hline 0 & 0,846 & 3,275 & 74,17 & 5135 \\
\hline
\end{tabular}

Tabela 6.27 - Resultados do $4^{\circ}$ problema com enriquecimento hierárquico

\begin{tabular}{c|c|c|c|c}
\hline \multicolumn{4}{c|}{ ENRIQUECIMENTO HIERÁRQUICO } \\
\hline $\begin{array}{c}\text { Dimensão da } \\
\text { base } \\
\text { enriquecedora }\end{array}$ & FIT final & $\begin{array}{c}\text { FIT } \\
\text { referência }\end{array}$ & $\begin{array}{c}\text { Erro } \\
\text { relativo } \\
(\%)\end{array}$ & $\begin{array}{c}\text { Graus de } \\
\text { liberdade } \\
\text { totais do } \\
P_{L}^{(k)}\end{array}$ \\
\hline 1 & 1,590 & 3,275 & 51,45 & 5159 \\
\hline 2 & 2,178 & 3,275 & 33,50 & 5183 \\
\hline 3 & 2,539 & 3,275 & 22,47 & 5207 \\
\hline 4 & 2,982 & 3,275 & 8,95 & 5231 \\
\hline 5 & 3,355 & 3,275 & 2,44 & 5255 \\
\hline 6 & 3,556 & 3,275 & 8,58 & 5279 \\
\hline
\end{tabular}

Tabela 6.28 - Resultados do $4^{\circ}$ problema com enriquecimento não-hierárquico

\begin{tabular}{c|c|c|c|c}
\hline \multicolumn{4}{c}{ ENRIQUECIMENTO NÃO-HIERÁRQUICO } \\
\hline $\begin{array}{c}\text { Dimensão da } \\
\text { base } \\
\text { enriquecedora }\end{array}$ & FIT final & $\begin{array}{c}\text { FIT } \\
\text { referência }\end{array}$ & $\begin{array}{c}\text { Erro } \\
\text { relativo } \\
(\%)\end{array}$ & $\begin{array}{c}\text { Graus de } \\
\text { liberdade } \\
\text { totais do } \\
P_{L}^{(k)}\end{array}$ \\
\hline 1 & 1,340 & 3,275 & 59,08 & 5159 \\
\hline 2 & 2,183 & 3,275 & 33,34 & 5183 \\
\hline 3 & 2,555 & 3,275 & 21,98 & 5207 \\
\hline 4 & 2,690 & 3,275 & 17,86 & 5231 \\
\hline 5 & 3,158 & 3,275 & 3,57 & 5255 \\
\hline 6 & 3,376 & 3,275 & 3,08 & 5279 \\
\hline
\end{tabular}


Tabela 6.29 - Resultados do $4^{\circ}$ problema com enriquecimento trigonométrico

\begin{tabular}{c|c|c|c|c}
\hline \multicolumn{5}{c}{ ENRIQUECIMENTO TRIGONOMÉTRICO } \\
\hline $\begin{array}{c}\text { Dimensão da } \\
\text { base } \\
\text { enriquecedora }\end{array}$ & FIT final & $\begin{array}{c}\text { FIT } \\
\text { referência }\end{array}$ & $\begin{array}{c}\text { Erro } \\
\text { relativo }(\%)\end{array}$ & $\begin{array}{c}\text { Graus de } \\
\text { liberdade } \\
\text { totais do } \\
P_{L}^{(k)}\end{array}$ \\
\hline 1 & 1,509 & 3,275 & 53,92 & 5159 \\
\hline 2 & 2,035 & 3,275 & 37,86 & 5183 \\
\hline 3 & 2,467 & 3,275 & 24,67 & 5207 \\
\hline 4 & 2,849 & 3,275 & 13,01 & 5231 \\
\hline 5 & 3,256 & 3,275 & 0,58 & 5255 \\
\hline 6 & 3,481 & 3,275 & 6,29 & 5279 \\
\hline
\end{tabular}

Analisando os dados das tabelas acima, verifica-se que o menor erro relativo para o FIT final é atingido quando se utiliza o enriquecimento trigonométrico (Tabela 6.29) com uma dimensão da base enriquecedora igual a cinco.

Comparando os resultados da Tabela 6.27 e Tabela 6.28, nota-se que o enriquecimento com a base não-hierárquica fornece resultados piores que os demais para esse caso. Finalmente, comparando os resultados da Tabela $6.26 \mathrm{com}$ as demais tabelas, verifica-se uma grande queda do erro relativo, comprovando a melhora na aproximação da solução ao utilizar o enriquecimento.

Observa-se, novamente, que ao empregar uma dimensão da base enriquecedora maior que cinco, o erro relativo volta a subir. Isso pode ser justificado pelo fato que o não atendimento do teste do "mosaico" está causando uma instabilidade na aproximação da solução.

\subsection{2 - Análise utilizando o MEFG}

Para a modelagem não foi utilizada a simetria horizontal do problema. Assim como na análise com a formulação híbrido-Trefftz, adotou-se a dimensão infinita da chapa como uma dimensão igual ao da chapa do subproblema $P_{G}^{(0)}$. Adotou-se um total de cinco termos de aproximação no problema. 
A rede de elementos finitos empregado nos cinco subproblemas $P_{L}^{(k)}$ pode ser visualizada na Figura 6.36. Ela contém um total de 1666 nós e 1573 elementos. 21 nós próximos a ponta da fissura foram enriquecidos em ambas as direções (x e y). As funções enriquecedoras são: $\mathrm{x}, \mathrm{x}^{2}, \mathrm{x}^{3}, F x$ na direção $\mathrm{x}$ e y, $\mathrm{y}^{2}, \mathrm{y}^{3}, F y$ na direção y. Contando com o enriquecimento, tem-se um total de 3500 graus de liberdade total.

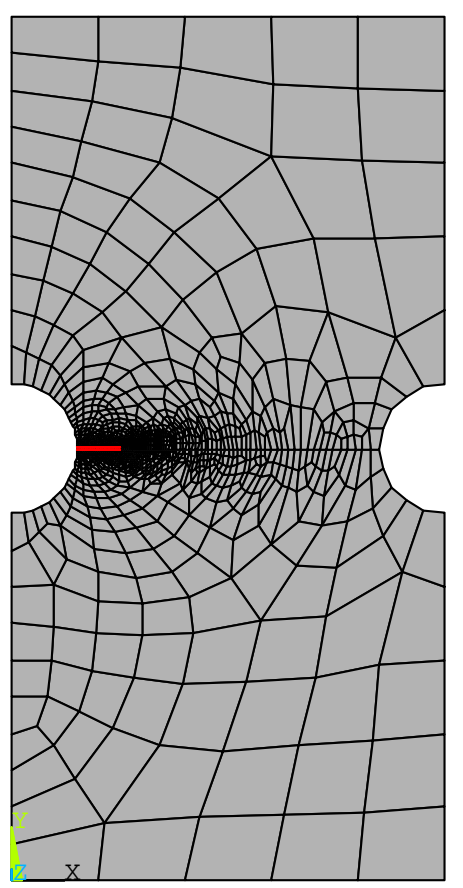

Figura 6.36 - Rede dos subproblemas $P_{L}^{(k)}$ do $4^{0}$ problema

A rede de elementos finitos utilizada nos subproblemas $P_{G}^{(0)}$ e $P_{G}^{(k)}$ é representada pela Figura 6.37. Ela contém um total de 436 nós e 408 elementos. Como não há presença de fissura, os dois subproblemas foram analisados utilizando o MEF clássico. 


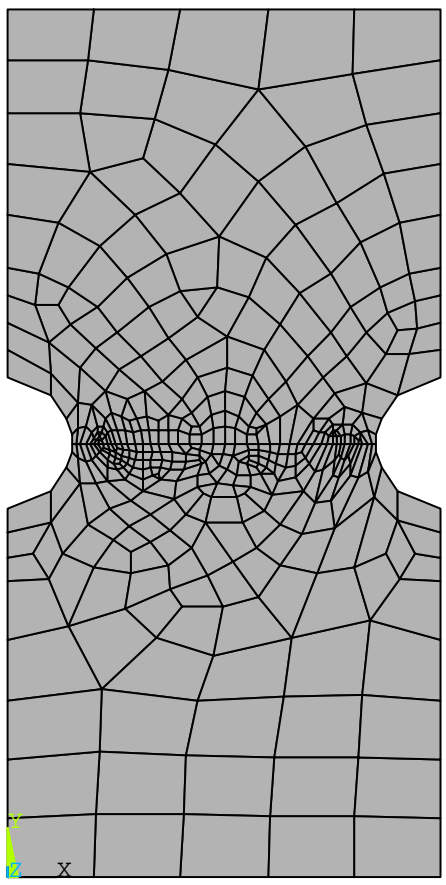

Figura 6.37 - Rede dos subproblemas $P_{G}^{(0)}$ e $P_{G}^{(k)}$ do $4^{0}$ problema

Os resultados dos FIT finais obtidos são apresentados na Tabela 6.30.

Tabela 6.30 - Resultados do $4^{\circ}$ problema com o MEFG

\begin{tabular}{c|c|c|c|c}
\hline Fissura & $\begin{array}{c}\text { FIT } \\
\text { referência }\end{array}$ & $\begin{array}{c}\text { FIT } \\
\text { calculado }\end{array}$ & $\begin{array}{c}\text { Erro } \\
\text { relativo } \\
(\%)\end{array}$ & $\begin{array}{c}\text { Graus de } \\
\text { liberdade } \\
\text { totais do } \\
P_{L}^{(k)}\end{array}$ \\
\hline 1 & 3,275 & 3,239 & 1,10 & \multirow{2}{*}{3500} \\
\hline 2 & 3,275 & 3,119 & 4,76 & \\
\hline
\end{tabular}

\section{6- Breve comentário sobre os resultados}

Observando os resultados dos problemas ilustrados nesse trabalho, nota-se que a formulação híbrido-Trefftz fornece valores de erros relativos mais baixos que o MEFG, além de permitir a utilização da simetria horizontal dos problemas, exigindo um menor número de graus de liberdade. 
No quarto problema, observa-se que o número de graus de liberdade utilizados no MEFG foi menor que na formulação híbrido-Trefftz, mesmo não explorando a simetria do problema. Porém, os valores dos erros relativos foram altos, principalmente para a segunda fissura. Talvez com uma rede mais refinada e/ou uma outra estratégia de enriquecimento esse erro relativo diminua e o número de graus de liberdade aumente.

Apesar disso, pode-se concluir que o emprego do MEFG no Método da Partição também proporciona bons resultados para esses problemas, porém eles podem ser melhorados. Uma maneira de reduzir erros de aproximação talvez seria a adimensionalização das funções para o enriquecimento, fazendo com que a aproximação independa do tamanho dos elementos, sendo, assim, mais eficiente. 


\section{7 - Considerações finais e conclusão}

O presente trabalho procurou fornecer uma contribuição à análise de problemas bidimensionais contendo múltiplas fissuras através do Método da Partição. Para isso, a fim de diminuir o custo computacional da análise sem comprometer o resultado final foram inseridas formulações não-convencionais de elementos finitos nos subproblemas em que a singularidade estava presente. As formulações para análise numérica empregadas neste trabalho foram a híbrido-Trefftz e o MEFG.

Ambas as formulações apresentaram um desempenho computacional melhor que o MEF clássico. Essa comparação foi feita levando-se em conta o número de graus de liberdade utilizados na análise.

Com a formulação híbrido-Trefftz, pôde-se explorar a simetria horizontal dos problemas. Assim, obteve-se um número de graus de liberdade menor que no MEFG, já que neste a simetria não foi explorada devido aos tipos de funções enriquecedoras adotados. Além disso, os valores dos erros relativos dos problemas ao utilizar a formulação híbrido-Trefftz foi menor que ao empregar o MEFG, demonstrando uma maior eficiência para aquela formulação. Isto, aliás, seria de se esperar, tendo-se em vista que a formulação de Trefftz explora a solução da equação de Navier.

Vale destacar que na formulação híbrido-Trefftz foram utilizadas funções polinomiais propostas por Muskhelishvili na aproximação do campo de tensões no domínio do elemento. Seria interessante testar alternativas de funções especiais, como as de Williams, utilizadas em Freitas e Ji (1996), para que a solução possa ser atingida com um número menor de elementos e ainda incrementar a precisão no cálculo dos fatores de intensidade de tensão.

Apesar de apresentar resultados piores em relação à outra formulação, o MEFG mostrou ser um método confiável para esses problemas. Essa diferença de precisão é relativa e pode ser consequência de diferentes fatores, como o fato de não terem sido empregadas funções enriquecedoras adimensionais, o que contribui para gerar certa sensibilidade na aproximação do problema a depender do tamanho do elemento a ser enriquecido. 
Finalmente, os resultados obtidos permitem projetar diferentes direções para a continuidade das investigações no tema. Entre elas destacam-se:

- O emprego de funções de Williams (FREITAS; JI, 1996), para a solução de Trefftz.

- Combinar o MEFG com a formulação híbrido-Trefftz na sua versão globallocal(DUARTE; KIM, 2008, DUARTE; KIM; BABUSKA, 2007.), que permite estender a análise de múltiplas fissuras em diferentes escalas.

- Realizar análises para o modo II de abertura de fissura.

- Analisar a propagação das fissuras.

- Implementar uma análise não-linear física na região próxima à fissura.

- Utilizar o recurso do processamento paralelo no Método da Partição a fím de comprovar a sua potencialidade no que diz respeito ao tempo de processamento. 


\section{Bibliografia}

ALIABADI, M. H., ROOKE, D. P. (1991). Numerical Fracture Mechanics. Southampton: Computational Mechanics Publications / Dordrecht: Kluwer Academic Publishers.

ALVES, M. M. (2010). Método da Partição na análise de múltiplas fissuras. Tese de Doutorado, Escola de Engenharia de São Carlos, Universidade de São Paulo.

Alves, M. M., ARGÔlo, H. S. D. de, \& PROENÇA, S. P. B. (2009). Método dos Elementos Finitos Generalizados na Análise Bidimensional de Sólidos com Múltiplas Fissuras. In: $30^{\circ}$ CILAMCE - Congresso Ibero Latino Americano de Métodos Computacionais em Engenharia, 2009, Armação de Búzios, RJ. Anais do 30 CILAMCE.

ANDERSSON, B., BABUŠKA, I., \& STEHLIN, P. (1998). Reliable Multiple-Site Damage Analysis of 3D Structures. FFA-TN-1998-18, The Aeronautical Research Institute of Sweden, Stockholm.

ASSAN, A. E. (2003). Método dos Elementos Finitos: primeiros passos. $2^{\mathrm{a}}$ ed., editora da UNICAMP. Campinas - SP.

BABUŠKA, I., \& ANDERSSON, B. (2005). The Splitting Method as a Tool for Multiple Damage Analysis. SIAM Journal on Scientific Computing., v. 26, n. 4, p. 1114-1145.

BARROS, F. B. (2002). Métodos Sem Rede e Método dos Elementos Finitos Generalizados em Análise Não-Linear de Estruturas. Tese de Doutrorado, Escola de Engenharia de São Carlos, Universidade de São Paulo.

BUECKNER, H. F. (1958). The Propagation of Cracks and the Energy of Elastic Deformation. ASME, Transaction, v.80, n. 6, p. 1225-1230. 
DUARTE, C. A., BABUŠKA, I., ODEN, J. T. (2000). Generalized finite element methods for three-dimensional structural mechanics problems. Computers and Structures, v. 77, p. 215232.

DUARTE, C. A., ODEN, J. T. (1996). An $h-p$ adaptive method using clouds. Computer Methods in Applied Mechanics and Engineering, v. 139, p. 237-262.

DUARTE, C. A., KIM, D. J. (2008). Analysis and applications of a generalized finite element method with global-local enrichment functions. Computer Methods in Applied Mechanics and Engineering, v. 197, p. 487-504.

DUARTE, C.A., KIM, D-J., BABUSKA, I. (2007). A Global-local Approach for the Construction of Enrichment Functions for the Generalized FEM and its Application to Threedimensional Cracks. In: ADVANCES IN MESHFREE TECHNIQUES. V.M.A. Leitão, C.J.S. Alves, Duarte, C.A., edts. Springer.

FREITAS, J. A. T. (1998). Formulation of elastostatic hybrid-Trefftz stress elements. Computer Methods in Applied Mechanics and Engineering, v. 153, p. 127-151.

FREITAS, J. A. T., ALMEIDA, J. P. B. M., PEREIRA, E. M. B. R. (1999). Non-conventional formulations for the finite element method. Computational Mechanics, v. 23, p. 488-501.

FREITAS, J. A. T., JI, Z. Y. (1996). Hybrid-Treffrz finite element formulation for simulation of singular stress fields. International Journal for Numerical Methods in Engineering, v. 39, p. 281-308.

GÓIS, W. (2004). Método dos elementos finitos generalizados em formulação variacional mista. Dissertação de Mestrado, Escola de Engenharia de São Carlos, Universidade de São Paulo. 
GÓIS, W. (2009). Elementos Finitos Híbridos e Híbrido-Mistos de Tensão com Enriquecimento Nodal. Tese de Doutorado, Escola de Engenharia de São Carlos, Universidade de São Paulo.

HOFFMAN, K., KUNZER, R. (1971). Álgebra Linear. Editora Polígono S.A.

JIROUSEK, J., LEON, N. (1977). A powerful finite element for plate bending. Computer Methods in Applied Mechanics and Engineering, v. 12, p. 77-96.

JIROUSEK, J. (1978). Basis for development of large finite elements locally satisfying all field equations. Computer Methods in Applied Mechanics and Engineering, v.14, p.65-92.

JIROUSEK, J., TEODORESCU, P. (1982). Large finite elements methods for the solution of problems in the theory of elasticity. Computers and Structures, v. 15, p. 575-587.

JIROUSEK, J., VENKATESH, A. (1992). Hybrid Trefftz plane elasticity elements with pmethod capabilities. Internacional Journal for Numerical Methods in Engineering, v. 35, p. 1443-1472.

JIROUSEK, J., WRÓBLEWSKI, A. (1996). T-elements: State of the Art and Future Trends. Archives of Computacional Methods in Engineering, v. 3, n. 4, p. 323-434.

LACHENBRUCH, A. H. (1961). Depth and Spacing of Tension Cracks. Journal of Geophysical Research, v. 66, n. 12, 4273-4292.

LEONEL, E. D. (2006). Método dos elementos de contorno aplicado à análise de corpos multi-fraturados. Dissertação de Mestrado, Escola de Engenharia de São Carlos, Universidade de São Paulo, São Carlos.

MELENK, J. M., BABUŠKA, I. (1996). The partition of unit finite element method: Basic theory and applications. Computer methods in applied mechanics and engineering, v. 139, p. 289-314. 
PETROLITO, J. (1996). Triangular thick plate elements based on a hybrid-Trefftz approach. Computers and Structures, v. 60, n. 6, p. 883-894.

PROENÇA, S. P. B. (2008). Introdução aos Métodos Numéricos. Notas de Aula. Escola de Engenharia de São Carlos, Universidade de São Paulo.

PROENÇA, S. P. B., TORRES, I. F. R. (2008). Generalized Finite Element Method for Nonlinear Three-Dimensional Analysis of Solids. International Journal of Computational Methods, v. 5, n. 1, p. 37-62.

SOUZA, C. O. de (2008). Formulação híbrido-Trefftz com enriquecimento seletivo: aplicação a problemas bidimensionais da elasticidade. Dissertação de Mestrado, Escola de Engenharia de São Carlos, Universidade de São Paulo, São Carlos.

SOUZA, C. O. de, PROENÇA, S. P. B. (2009). A hybrid-Trefftz formulation for plane elasticity with selective enrichment of the approximations. Communications in Numerical Methods in Engineering. Disponível em: www.interscience.wiley.com.

STROUBOULIS, T., BABUŠKA, I., COPPS, K. (2000). The design and analysis of the Generalized Finite Element Method. Computer Methods in Applied Mechanics and Engineering, v. 181, p. 43-69.

TIMOSHENKO, S. P., GOODIER, J. N. (1980). Teoria da Elasticidade. Guanabara Dois S.A.

TORRES, I. F. R., (2003). Desenvolvimento e aplicação do método dos elementos finitos generalizados em análise tridimensional não-linear de sólidos. Tese de Doutorado, Escola de Engenharia de São Carlos, Universidade de São Paulo, São Carlos.

ZIENKIEWICZ, O. C., QU, S., TAYLOR, R. L., NAKAZAWA, S. (1986). The patch test for mixed formulations. International Journal for Numerical Methods in Engineering, v. 23, p. $1873-1883$. 


\section{Anexo A - Matrizes utilizadas na aproximação dos campos de deslocamentos e tensões em domínios contínuos: Construção a partir da Equação de Navier}

As matrizes apresentadas a seguir foram retiradas de SOUZA (2008).

\section{- Matriz $\mathrm{N}_{\mathrm{c}}$ :}

Da $1^{\mathrm{a}}$ à $9^{\mathrm{a}}$ coluna:

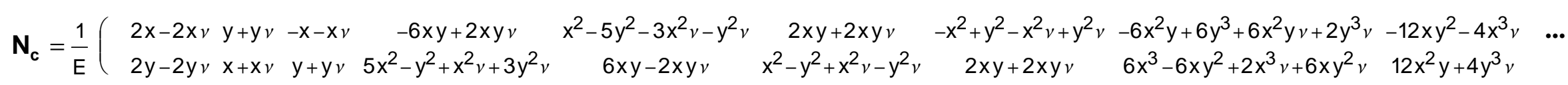

Da $10^{\mathrm{a}}$ à $13^{\mathrm{a}}$ coluna :

$$
\begin{array}{lllc}
3 x^{2} y-y^{3}+3 x^{2} y v-y^{3} v & -x^{3}+3 x y^{2}-x^{3} v+3 x y^{2} v & -4 x^{3} y+20 x y^{3}+12 x^{3} y v+4 x y^{3} v & -x^{4}-18 x^{2} y^{2}+7 y^{4}-5 x^{4} v+6 x^{2} y^{2} v+3 \ldots \\
x^{3}-3 x y^{2}+x^{3} v-3 x y^{2} v & 3 x^{2} y-y^{3}+3 x^{2} y v-y^{3} v & 7 x^{4}-18 x^{2} y^{2}-y^{4}+3 x^{4} v+6 x^{2} y^{2} v-5 y^{4} v & 20 x^{3} y-4 x y^{3}+4 x^{3} y v+12 x y^{3} v
\end{array}
$$

Da $14^{\mathrm{a}}$ à $17^{\mathrm{a}}$ coluna:

$$
\begin{array}{cccc}
4 \mathrm{x}^{3} \mathrm{y}-4 \mathrm{x} \mathrm{y}^{3}+4 \mathrm{x}^{3} \mathrm{y} v-4 \mathrm{xy}^{3} v & -\mathrm{x}^{4}+6 \mathrm{x}^{2} \mathrm{y}^{2}-\mathrm{y}^{4}-\mathrm{x}^{4} v+6 \mathrm{x}^{2} \mathrm{y}^{2} v-\mathrm{y}^{4} v & 40 \mathrm{x}^{2} \mathrm{y}^{3}-8 \mathrm{y}^{5}+20 \mathrm{x}^{4} \mathrm{y} v-4 \mathrm{y}^{5} v & -2 \mathrm{x}^{5}-20 \mathrm{x}^{3} \mathrm{y}^{2}+30 \mathrm{x} \mathrm{y}^{4}-6 \mathrm{x}^{5} v+20 \mathrm{x}^{3} \mathrm{y}^{2} v+10 \mathrm{x} . . . \\
\mathrm{x}^{4}-6 \mathrm{x}^{2} \mathrm{y}^{2}+\mathrm{y}^{4}+\mathrm{x}^{4} v-6 \mathrm{x}^{2} \mathrm{y}^{2} v+\mathrm{y}^{4} v & 4 \mathrm{x}^{3} \mathrm{y}-4 \mathrm{x} \mathrm{y}^{3}+4 \mathrm{x}^{3} \mathrm{y} v-4 \mathrm{xy}^{3} v & 8 \mathrm{x}^{5}-40 \mathrm{x}^{3} \mathrm{y}^{2}+4 \mathrm{x}^{5} v-20 \mathrm{x} \mathrm{y}^{4} v & 30 \mathrm{x}^{4} \mathrm{y}-20 \mathrm{x}^{2} \mathrm{y}^{3}-2 \mathrm{y}^{5}+10 \mathrm{x}^{4} \mathrm{y} v+20 \mathrm{x}^{2} \mathrm{y}^{3} v-6 !
\end{array}
$$


Da $18^{\mathrm{a}}$ à $20^{\mathrm{a}}$ coluna:

$$
\begin{aligned}
& 5 \mathrm{x}^{4} \mathrm{y}-10 \mathrm{x}^{2} \mathrm{y}^{3}+\mathrm{y}^{5}+5 \mathrm{x}^{4} \mathrm{y} v-10 \mathrm{x}^{2} \mathrm{y}^{3} v+\mathrm{y}^{5} v-\mathrm{x}^{5}+10 \mathrm{x}^{3} \mathrm{y}^{2}-5 \mathrm{x} \mathrm{y}^{4}-\mathrm{x}^{5} v+10 \mathrm{x}^{3} \mathrm{y}^{2} v-5 \mathrm{x} \mathrm{y}^{4} v \quad 6 \mathrm{x}^{5} \mathrm{y}+60 \mathrm{x}^{3} \mathrm{y}^{3}-42 \mathrm{x} \mathrm{y}^{5}+30 \mathrm{x}^{5} \mathrm{y} v-20 \mathrm{x}^{3} \mathrm{y}^{3} v-18 \mathrm{x} \mathrm{y}^{5} v \\
& \mathrm{x}^{5}-10 \mathrm{x}^{3} \mathrm{y}^{2}+5 \mathrm{x} \mathrm{y}^{4}+\mathrm{x}^{5} v-10 \mathrm{x}^{3} \mathrm{y}^{2} v+5 \mathrm{x} \mathrm{y}^{4} v \quad 5 \mathrm{x}^{4} \mathrm{y}-10 \mathrm{x}^{2} \mathrm{y}^{3}+\mathrm{y}^{5}+5 \mathrm{x}^{4} \mathrm{y} v-10 \mathrm{x}^{2} \mathrm{y}^{3} v+\mathrm{y}^{5} v \quad 9 \mathrm{x}^{6}-75 \mathrm{x}^{4} \mathrm{y}^{2}+15 \mathrm{x}^{2} \mathrm{y}^{4}+3 \mathrm{y}^{6}+5 \mathrm{x}^{6} v-15 \mathrm{x}^{4} \mathrm{y}^{2} v-45 \mathrm{x}^{2} \mathrm{y}^{4} v+7 \mathrm{y}^{6} v
\end{aligned}
$$

$21^{\mathrm{a}}$ e $22^{\mathrm{a}}$ colunas:

$$
\begin{array}{cr}
-3 x^{6}-15 x^{4} y^{2}+75 x^{2} y^{4}-9 y^{6}-7 x^{6} v+45 x^{4} y^{2} v+15 x^{2} y^{4} v-5 y^{6} v & 6 x^{5} y-20 x^{3} y^{3}+6 x y^{5}+6 x^{5} y v-20 x^{3} y^{3} v+6 x y^{5} v \ldots \\
42 x^{5} y-60 x^{3} y^{3}-6 x y^{5}+18 x^{5} y v+20 x^{3} y^{3} v-30 x y^{5} v & x^{6}-15 x^{4} y^{2}+15 x^{2} y^{4}-y^{6}+x^{6} v-15 x^{4} y^{2} v+15 x^{2} y^{4} v-
\end{array}
$$

$23^{a}$ e $24^{a}$ colunas:

$$
\begin{array}{cc}
-\mathrm{x}^{6}+15 \mathrm{x}^{4} \mathrm{y}^{2}-15 \mathrm{x}^{2} \mathrm{y}^{4}+\mathrm{y}^{6}-\mathrm{x}^{6} v+15 \mathrm{x}^{4} \mathrm{y}^{2} v-15 \mathrm{x}^{2} \mathrm{y}^{4} v+\mathrm{y}^{6} v & 14 \mathrm{x}^{6} \mathrm{y}+70 \mathrm{x}^{4} \mathrm{y}^{3}-126 \mathrm{x}^{2} \mathrm{y}^{5}+10 \mathrm{y}^{7}+42 \mathrm{x}^{6} \mathrm{y} v-70 \mathrm{x}^{4} \mathrm{y}^{3} v-42 \mathrm{x}^{2} \mathrm{y}^{5} v+6 \ldots \\
6 \mathrm{x}^{5} \mathrm{y}-20 \mathrm{x}^{3} \mathrm{y}^{3}+6 \mathrm{x} \mathrm{y}^{5}+6 \mathrm{x}^{5} \mathrm{y} v-20 \mathrm{x}^{3} \mathrm{y}^{3} v+6 \mathrm{x} \mathrm{y}^{5} v & 10 \mathrm{x}^{7}-126 \mathrm{x}^{5} \mathrm{y}^{2}+70 \mathrm{x}^{3} \mathrm{y}^{4}+14 \mathrm{x} \mathrm{y}^{6}+6 \mathrm{x}^{7} v-42 \mathrm{x}^{5} \mathrm{y}^{2} v-70 \mathrm{x}^{3} \mathrm{y}^{4} v+42 \mathrm{x}
\end{array}
$$

$25^{\mathrm{a}}$ e $26^{\mathrm{a}}$ colunas:

$$
\begin{array}{ll}
-4 \mathrm{x}^{7}+140 \mathrm{x}^{3} \mathrm{y}^{4}-56 \mathrm{x} \mathrm{y}^{6}-8 \mathrm{x}^{7} v+84 \mathrm{x}^{5} \mathrm{y}^{2} v-28 \mathrm{x} \mathrm{y}^{6} v & 7 \mathrm{x}^{6} \mathrm{y}-35 \mathrm{x}^{4} \mathrm{y}^{3}+21 \mathrm{x}^{2} \mathrm{y}^{5}-\mathrm{y}^{7}+7 \mathrm{x}^{6} \mathrm{y} v-35 \mathrm{x}^{4} \mathrm{y}^{3} v+21 \mathrm{x}^{2} \mathrm{y}^{5} v-\ldots \\
56 \mathrm{x}^{6} \mathrm{y}-140 \mathrm{x}^{4} \mathrm{y}^{3}+4 \mathrm{y}^{7}+28 \mathrm{x}^{6} \mathrm{y} v-84 \mathrm{x}^{2} \mathrm{y}^{5} v+8 \mathrm{y}^{7} v & \mathrm{x}^{7}-21 \mathrm{x}^{5} \mathrm{y}^{2}+35 \mathrm{x}^{3} \mathrm{y}^{4}-7 \mathrm{x} \mathrm{y}^{6}+\mathrm{x}^{7} v-21 \mathrm{x}^{5} \mathrm{y}^{2} v+35 \mathrm{x}^{3} \mathrm{y}^{4} v-7 \mathrm{x}
\end{array}
$$

\section{$27^{\mathrm{a}}$ e $28^{\mathrm{a}}$ colunas:}

$-x^{7}+21 x^{5} y^{2}-35 x^{3} y^{4}+7 x y^{6}-x^{7} v+21 x^{5} y^{2} v-35 x^{3} y^{4} v+7 x y^{6} v \quad 24 x^{7} y+56 x^{5} y^{3}-280 x^{3} y^{5}+72 x y^{7}+56 x^{7} y v-168 x^{5} y^{3} v-56 x^{3} y^{5} v+40 x y^{7} v$ $7 \mathrm{x}^{6} \mathrm{y}-35 \mathrm{x}^{4} \mathrm{y}^{3}+21 \mathrm{x}^{2} \mathrm{y}^{5}-\mathrm{y}^{7}+7 \mathrm{x}^{6} \mathrm{y} v-35 \mathrm{x}^{4} \mathrm{y}^{3} v+21 \mathrm{x}^{2} \mathrm{y}^{5} v-\mathrm{y}^{7} v \quad 11 \mathrm{x}^{8}-196 \mathrm{x}^{6} \mathrm{y}^{2}+210 \mathrm{x}^{4} \mathrm{y}^{4}+28 \mathrm{x}^{2} \mathrm{y}^{6}-5 \mathrm{y}^{8}+7 \mathrm{x}^{8} v-84 \mathrm{x}^{6} \mathrm{y}^{2} v-70 \mathrm{x}^{4} \mathrm{y}^{4} v+140 \mathrm{x}^{2} \mathrm{y}^{6} v-9 \mathrm{y}^{8} v$ 
$29^{\mathrm{a}}$ e $30^{\mathrm{a}}$ colunas:

$-5 \mathrm{x}^{8}+28 \mathrm{x}^{6} \mathrm{y}^{2}+210 \mathrm{x}^{4} \mathrm{y}^{4}-196 \mathrm{x}^{2} \mathrm{y}^{6}+11 \mathrm{y}^{8}-9 \mathrm{x}^{8} v+140 \mathrm{x}^{6} \mathrm{y}^{2} v-70 \mathrm{x}^{4} \mathrm{y}^{4} v-84 \mathrm{x}^{2} \mathrm{y}^{6} v+7 \mathrm{y}^{8} v \quad 8 \mathrm{x}^{7} \mathrm{y}-56 \mathrm{x}^{5} \mathrm{y}^{3}+56 \mathrm{x}^{3} \mathrm{y}^{5}-8 \mathrm{x} \mathrm{y}^{7}+8 \mathrm{x}^{7} \mathrm{y} v-56 \mathrm{x}^{5} \mathrm{y}^{3} v+56 \mathrm{x}^{3} \mathrm{y}^{5} v-8 \mathrm{x} \mathrm{y}^{7} v \quad \ldots$ $72 x^{7} y-280 x^{5} y^{3}+56 x^{3} y^{5}+24 x y^{7}+40 x^{7} y v-56 x^{5} y^{3} v-168 x^{3} y^{5} v+56 x y^{7} v \quad x^{8}-28 x^{6} y^{2}+70 x^{4} y^{4}-28 x^{2} y^{6}+y^{8}+x^{8} v-28 x^{6} y^{2} v+70 x^{4} y^{4} v-28 x^{2} y^{6} v+y^{8} v$

$31^{\mathrm{a}}$ e $32^{\mathrm{a}}$ colunas:

$-\mathrm{x}^{8}+28 \mathrm{x}^{6} \mathrm{y}^{2}-70 \mathrm{x}^{4} \mathrm{y}^{4}+28 \mathrm{x}^{2} \mathrm{y}^{6}-\mathrm{y}^{8}-\mathrm{x}^{8} v+28 \mathrm{x}^{6} \mathrm{y}^{2} v-70 \mathrm{x}^{4} \mathrm{y}^{4} v+28 \mathrm{x}^{2} \mathrm{y}^{6} v-\mathrm{y}^{8} v 36 \mathrm{x}^{8} \mathrm{y}-504 \mathrm{x}^{4} \mathrm{y}^{5}+288 \mathrm{x}^{2} \mathrm{y}^{7}-12 \mathrm{y}^{9}+72 \mathrm{x}^{8} \mathrm{y} v-336 \mathrm{x}^{6} \mathrm{y}^{3} v+144 \mathrm{x}^{2} \mathrm{y}^{7} v-8 \mathrm{y}^{9} v$ $8 x^{7} y-56 x^{5} y^{3}+56 x^{3} y^{5}-8 x y^{7}+8 x^{7} y v-56 x^{5} y^{3} v+56 x^{3} y^{5} v-8 x y^{7} v \quad 12 x^{9}-288 x^{7} y^{2}+504 x^{5} y^{4}-36 x y^{8}+8 x^{9} v-144 x^{7} y^{2} v+336 x^{3} y^{6} v-72 x y^{8} v$

$33^{\mathrm{a}}$ coluna:

$-6 x^{9}+72 x^{7} y^{2}+252 x^{5} y^{4}-504 x^{3} y^{6}+90 x y^{8}-10 x^{9} v+216 x^{7} y^{2} v-252 x^{5} y^{4} v-168 x^{3} y^{6} v+54 x y^{8} v$ $90 x^{8} y-504 x^{6} y^{3}+252 x^{4} y^{5}+72 x^{2} y^{7}-6 y^{9}+54 x^{8} y v-168 x^{6} y^{3} v-252 x^{4} y^{5} v+216 x^{2} y^{7} v-10 y^{9} v$

$34^{\mathrm{a}}$ coluna:

$9 \mathrm{x}^{8} \mathrm{y}-84 \mathrm{x}^{6} \mathrm{y}^{3}+126 \mathrm{x}^{4} \mathrm{y}^{5}-36 \mathrm{x}^{2} \mathrm{y}^{7}+\mathrm{y}^{9}+9 \mathrm{x}^{8} \mathrm{y} v-84 \mathrm{x}^{6} \mathrm{y}^{3} v+126 \mathrm{x}^{4} \mathrm{y}^{5} v-36 \mathrm{x}^{2} \mathrm{y}^{7} v+\mathrm{y}^{9} v$ $\mathrm{x}^{9}-36 \mathrm{x}^{7} \mathrm{y}^{2}+126 \mathrm{x}^{5} \mathrm{y}^{4}-84 \mathrm{x}^{3} \mathrm{y}^{6}+9 \mathrm{x} \mathrm{y}^{8}+\mathrm{x}^{9} v-36 \mathrm{x}^{7} \mathrm{y}^{2} v+126 \mathrm{x}^{5} \mathrm{y}^{4} v-84 \mathrm{x}^{3} \mathrm{y}^{6} v+9 \mathrm{x} \mathrm{y}^{8} v$

$35^{\text {a }}$ coluna:

$-\mathrm{x}^{9}+36 \mathrm{x}^{7} \mathrm{y}^{2}-126 \mathrm{x}^{5} \mathrm{y}^{4}+84 \mathrm{x}^{3} \mathrm{y}^{6}-9 \mathrm{x} \mathrm{y}^{8}-\mathrm{x}^{9} v+36 \mathrm{x}^{7} \mathrm{y}^{2} v-126 \mathrm{x}^{5} \mathrm{y}^{4} v+84 \mathrm{x}^{3} \mathrm{y}^{6} v-9 \mathrm{x} \mathrm{y}^{8} v$ $9 x^{8} y-84 x^{6} y^{3}+126 x^{4} y^{5}-36 x^{2} y^{7}+y^{9}+9 x^{8} y v-84 x^{6} y^{3} v+126 x^{4} y^{5} v-36 x^{2} y^{7} v+y^{9} v$ 
$36^{\text {a coluna: }}$

$50 x^{9} y-120 x^{7} y^{3}-756 x^{5} y^{5}+840 x^{3} y^{7}-110 x y^{9}+90 x^{9} y v-600 x^{7} y^{3} v+252 x^{5} y^{5} v+360 x^{3} y^{7} v-70 x y^{9} v$

$13 x^{10}-405 x^{8} y^{2}+1050 x^{6} y^{4}-210 x^{4} y^{6}-135 x^{2} y^{8}+7 y^{10}+9 x^{10} v-225 x^{8} y^{2} v+210 x^{6} y^{4} v+630 x^{4} y^{6} v-315 x^{2} y^{8} v+11 y^{10} v$

$37^{\mathrm{a}}$ coluna:

$$
\begin{gathered}
-7 x^{10}+135 x^{8} y^{2}+210 x^{6} y^{4}-1050 x^{4} y^{6}+405 x^{2} y^{8}-13 y^{10}-11 x^{10} v+315 x^{8} y^{2} v-630 x^{6} y^{4} v-210 x^{4} y^{6} v+225 x^{2} y^{8} v-9 y^{10} v \\
110 x^{9} y-840 x^{7} y^{3}+756 x^{5} y^{5}+120 x^{3} y^{7}-50 x y^{9}+70 x^{9} y v-360 x^{7} y^{3} v-252 x^{5} y^{5} v+600 x^{3} y^{7} v-90 x y^{9} v
\end{gathered}
$$

$38^{a}$ coluna:

$10 x^{9} y-120 x^{7} y^{3}+252 x^{5} y^{5}-120 x^{3} y^{7}+10 x y^{9}+10 x^{9} y v-120 x^{7} y^{3} v+252 x^{5} y^{5} v-120 x^{3} y^{7} v+10 x y^{9} v$ $\mathrm{x}^{10}-45 \mathrm{x}^{8} \mathrm{y}^{2}+210 \mathrm{x}^{6} \mathrm{y}^{4}-210 \mathrm{x}^{4} \mathrm{y}^{6}+45 \mathrm{x}^{2} \mathrm{y}^{8}-\mathrm{y}^{10}+\mathrm{x}^{10} v-45 \mathrm{x}^{8} \mathrm{y}^{2} v+210 \mathrm{x}^{6} \mathrm{y}^{4} v-210 \mathrm{x}^{4} \mathrm{y}^{6} v+45 \mathrm{x}^{2} \mathrm{y}^{8} v-\mathrm{y}^{10} v$

$39^{a}$ coluna:

$$
\begin{gathered}
-x^{10}+45 x^{8} y^{2}-210 x^{6} y^{4}+210 x^{4} y^{6}-45 x^{2} y^{8}+y^{10}-x^{10} v+45 x^{8} y^{2} v-210 x^{6} y^{4} v+210 x^{4} y^{6} v-45 x^{2} y^{8} v+y^{10} v \\
10 x^{9} y-120 x^{7} y^{3}+252 x^{5} y^{5}-120 x^{3} y^{7}+10 x y^{9}+10 x^{9} y v-120 x^{7} y^{3} v+252 x^{5} y^{5} v-120 x^{3} y^{7} v+10 x y^{9} v
\end{gathered}
$$

$40^{a}$ coluna:

$66 x^{10} y-330 x^{8} y^{3}-924 x^{6} y^{5}+1980 x^{4} y^{7}-550 x^{2} y^{9}+14 y^{11}+110 x^{10} y v-990 x^{8} y^{3} v+924 x^{6} y^{5} v+660 x^{4} y^{7} v-330 x^{2} y^{9} v+10 y^{11} v$ $14 x^{11}-550 x^{9} y^{2}+1980 x^{7} y^{4}-924 x^{5} y^{6}-330 x^{3} y^{8}+66 x y^{10}+10 x^{11} v-330 x^{9} y^{2} v+660 x^{7} y^{4} v+924 x^{5} y^{6} v-990 x^{3} y^{8} v+110 x y^{10} v$ 
$41^{\mathrm{a}}$ coluna:

$-8 x^{11}+220 x^{9} y^{2}-1848 x^{5} y^{6}+1320 x^{3} y^{8}-132 x y^{10}-12 x^{11} v+440 x^{9} y^{2} v-1320 x^{7} y^{4} v+660 x^{3} y^{8} v-88 x y^{10} v$ $132 x^{10} y-1320 x^{8} y^{3}+1848 x^{6} y^{5}-220 x^{2} y^{9}+8 y^{11}+88 x^{10} y v-660 x^{8} y^{3} v+1320 x^{4} y^{7} v-440 x^{2} y^{9} v+12 y^{11} v$

$42^{\mathrm{a}}$ coluna:

$11 x^{10} y-165 x^{8} y^{3}+462 x^{6} y^{5}-330 x^{4} y^{7}+55 x^{2} y^{9}-y^{11}+11 x^{10} y v-165 x^{8} y^{3} v+462 x^{6} y^{5} v-330 x^{4} y^{7} v+55 x^{2} y^{9} v-y^{11} v$ $x^{11}-55 x^{9} y^{2}+330 x^{7} y^{4}-462 x^{5} y^{6}+165 x^{3} y^{8}-11 x y^{10}+x^{11} v-55 x^{9} y^{2} v+330 x^{7} y^{4} v-462 x^{5} y^{6} v+165 x^{3} y^{8} v-11 x y^{10} v$

$43^{\mathrm{a}}$ coluna:

$-x^{11}+55 x^{9} y^{2}-330 x^{7} y^{4}+462 x^{5} y^{6}-165 x^{3} y^{8}+11 x y^{10}-x^{11} v+55 x^{9} y^{2} v-330 x^{7} y^{4} v+462 x^{5} y^{6} v-165 x^{3} y^{8} v+11 x y^{10} v$ $11 x^{10} y-165 x^{8} y^{3}+462 x^{6} y^{5}-330 x^{4} y^{7}+55 x^{2} y^{9}-y^{11}+11 x^{10} y v-165 x^{8} y^{3} v+462 x^{6} y^{5} v-330 x^{4} y^{7} v+55 x^{2} y^{9} v-y^{11} v$

44 coluna:

$84 x^{11} y-660 x^{9} y^{3}-792 x^{7} y^{5}+3960 x^{5} y^{7}-1980 x^{3} y^{9}+156 x y^{11}+132 x^{11} y v-1540 x^{9} y^{3} v+2376 x^{7} y^{5} v+792 x^{5} y^{7} v-1100 x^{3} y^{9} v+108 x y^{11} v$ $15 x^{12}-726 x^{10} y^{2}+3465 x^{8} y^{4}-2772 x^{6} y^{6}-495 x^{4} y^{8}+330 x^{2} y^{10}-9 y^{12}+11 x^{12} v-462 x^{10} y^{2} v+1485 x^{8} y^{4} v+924 x^{6} y^{6} v-2475 x^{4} y^{8} v+594 x^{2} y^{10} v-13 y^{12} v$

$45^{\mathrm{a}}$ coluna:

$-9 x^{12}+330 x^{10} y^{2}-495 x^{8} y^{4}-2772 x^{6} y^{6}+3465 x^{4} y^{8}-726 x^{2} y^{10}+15 y^{12}-13 x^{12} v+594 x^{10} y^{2} v-2475 x^{8} y^{4} v+924 x^{6} y^{6} v+1485 x^{4} y^{8} v-462 x^{2} y^{10} v+11 y^{12} v$ $156 x^{11} y-1980 x^{9} y^{3}+3960 x^{7} y^{5}-792 x^{5} y^{7}-660 x^{3} y^{9}+84 x y^{11}+108 x^{11} y v-1100 x^{9} y^{3} v+792 x^{7} y^{5} v+2376 x^{5} y^{7} v-1540 x^{3} y^{9} v+132 x y^{11} v$ 
$46^{\mathrm{a}}$ coluna:

$$
\begin{gathered}
12 x^{11} y-220 x^{9} y^{3}+792 x^{7} y^{5}-792 x^{5} y^{7}+220 x^{3} y^{9}-12 x y^{11}+12 x^{11} y v-220 x^{9} y^{3} v+792 x^{7} y^{5} v-792 x^{5} y^{7} v+220 x^{3} y^{9} v-12 x y^{11} v \\
x^{12}-66 x^{10} y^{2}+495 x^{8} y^{4}-924 x^{6} y^{6}+495 x^{4} y^{8}-66 x^{2} y^{10}+y^{12}+x^{12} v-66 x^{10} y^{2} v+495 x^{8} y^{4} v-924 x^{6} y^{6} v+495 x^{4} y^{8} v-66 x^{2} y^{10} v+y^{12} v
\end{gathered}
$$

47 coluna:

$$
\left.\begin{array}{c}
-x^{12}+66 x^{10} y^{2}-495 x^{8} y^{4}+924 x^{6} y^{6}-495 x^{4} y^{8}+66 x^{2} y^{10}-y^{12}-x^{12} v+66 x^{10} y^{2} v-495 x^{8} y^{4} v+924 x^{6} y^{6} v-495 x^{4} y^{8} v+66 x^{2} y^{10} v-y^{12} v \\
12 x^{11} y-220 x^{9} y^{3}+792 x^{7} y^{5}-792 x^{5} y^{7}+220 x^{3} y^{9}-12 x y^{11}+12 x^{11} y v-220 x^{9} y^{3} v+792 x^{7} y^{5} v-792 x^{5} y^{7} v+220 x^{3} y^{9} v-12 x y^{11} v
\end{array}\right)
$$

\section{- Matriz $S_{c}: S_{c}=D L_{c}{ }^{\top} N_{c}$}

Da $1^{\mathrm{a}}$ à $16^{\mathrm{a}}$ coluna:

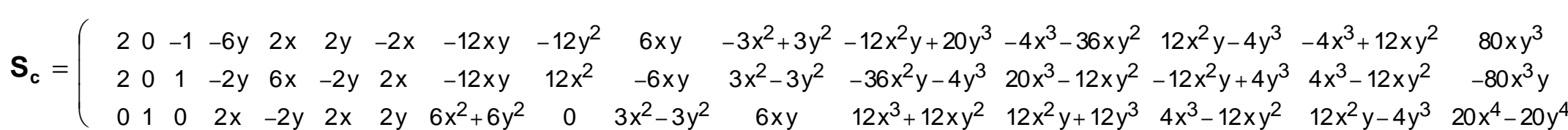

Da $17^{\mathrm{a}}$ à $22^{\mathrm{a}}$ coluna:

$$
\begin{array}{cccccc}
-10 x^{4}-60 x^{2} y^{2}+30 y^{4} & 20 x^{3} y-20 x y^{3} & -5 x^{4}+30 x^{2} y^{2}-5 y^{4} & 30 x^{4} y+180 x^{2} y^{3}-42 y^{5} & -18 x^{5}-60 x^{3} y^{2}+150 x y^{4} & 30 x^{4} y-60 x^{2} y^{3}+6 y^{5} \\
30 x^{4}-60 x^{2} y^{2}-10 y^{4} & -20 x^{3} y+20 x y^{3} & 5 x^{4}-30 x^{2} y^{2}+5 y^{4} & -150 x^{4} y+60 x^{2} y^{3}+18 y^{5} & 42 x^{5}-180 x^{3} y^{2}-30 x y^{4} & -30 x^{4} y+60 x^{2} y^{3}-6 y^{5} \\
40 x^{3} y+40 x y^{3} & 5 x^{4}-30 x^{2} y^{2}+5 y^{4} & 20 x^{3} y-20 x y^{3} & 30 x^{5}-60 x^{3} y^{2}-90 x y^{4} & 90 x^{4} y+60 x^{2} y^{3}-30 y^{5} & 6 x^{5}-60 x^{3} y^{2}+30 x y^{4}
\end{array}
$$


Da $23^{\mathrm{a}}$ à $27^{\mathrm{a}}$ coluna:

$$
\begin{array}{ccccc}
-6 x^{5}+60 x^{3} y^{2}-30 x y^{4} & 84 x^{5} y+280 x^{3} y^{3}-252 x y^{5} & -28 x^{6}+420 x^{2} y^{4}-56 y^{6} & 42 x^{5} y-140 x^{3} y^{3}+42 x y^{5} & -7 x^{6}+105 x^{4} y^{2}-105 x^{2} y^{4}+7 y^{6} \\
6 x^{5}-60 x^{3} y^{2}+30 x y^{4} & -252 x^{5} y+280 x^{3} y^{3}+84 x y^{5} & 56 x^{6}-420 x^{4} y^{2}+28 y^{6} & -42 x^{5} y+140 x^{3} y^{3}-42 x y^{5} & 7 x^{6}-105 x^{4} y^{2}+105 x^{2} y^{4}-7 y^{6} \\
30 x^{4} y-60 x^{2} y^{3}+6 y^{5} & 42 x^{6}-210 x^{4} y^{2}-210 x^{2} y^{4}+42 y^{6} & 168 x^{5} y-168 x y^{5} & 7 x^{6}-105 x^{4} y^{2}+105 x^{2} y^{4}-7 y^{6} & 42 x^{5} y-140 x^{3} y^{3}+42 x y^{5}
\end{array}
$$

Da $28^{\mathrm{a}}$ à $31^{\mathrm{a}}$ coluna:

$168 x^{6} y+280 x^{4} y^{3}-840 x^{2} y^{5}+72 y^{7}-40 x^{7}+168 x^{5} y^{2}+840 x^{3} y^{4}-392 x y^{6} \quad 56 x^{6} y-280 x^{4} y^{3}+168 x^{2} y^{5}-8 y^{7}-8 x^{7}+168 x^{5} y^{2}-280 x^{3} y^{4}+56 x y^{6}$ $-392 x^{6} y+840 x^{4} y^{3}+168 x^{2} y^{5}-40 y^{7} \quad 72 x^{7}-840 x^{5} y^{2}+280 x^{3} y^{4}+168 x y^{6}-56 x^{6} y+280 x^{4} y^{3}-168 x^{2} y^{5}+8 y^{7} \quad 8 x^{7}-168 x^{5} y^{2}+280 x^{3} y^{4}-56 x y^{6}$ $56 x^{7}-504 x^{5} y^{2}-280 x^{3} y^{4}+280 x y^{6} \quad 280 x^{6} y-280 x^{4} y^{3}-504 x^{2} y^{5}+56 y^{7} \quad 8 x^{7}-168 x^{5} y^{2}+280 x^{3} y^{4}-56 x y^{6} \quad 56 x^{6} y-280 x^{4} y^{3}+168 x^{2} y^{5}-8 y^{7}$

Da $32^{\mathrm{a}}$ à $35^{\mathrm{a}}$ coluna:

$288 x^{7} y-2016 x^{3} y^{5}+576 x y^{7} \quad-54 x^{8}+504 x^{6} y^{2}+1260 x^{4} y^{4}-1512 x^{2} y^{6}+90 y^{8} \quad 72 x^{7} y-504 x^{5} y^{3}+504 x^{3} y^{5}-72 x y^{7} \quad-9 x^{8}+252 x^{6} y^{2}-630 x^{4} y^{4}+252 x^{2} y^{6}-9 y^{8} \quad \ldots$ $-576 x^{7} y+2016 x^{5} y^{3}-288 x y^{7} \quad 90 x^{8}-1512 x^{6} y^{2}+1260 x^{4} y^{4}+504 x^{2} y^{6}-54 y^{8} \quad-72 x^{7} y+504 x^{5} y^{3}-504 x^{3} y^{5}+72 x y^{7} \quad 9 x^{8}-252 x^{6} y^{2}+630 x^{4} y^{4}-252 x^{2} y^{6}+9 y^{8}$ $72 x^{8}-1008 x^{6} y^{2}+1008 x^{2} y^{6}-72 y^{8} \quad 432 x^{7} y-1008 x^{5} y^{3}-1008 x^{3} y^{5}+432 x y^{7} \quad 9 x^{8}-252 x^{6} y^{2}+630 x^{4} y^{4}-252 x^{2} y^{6}+9 y^{8} \quad 72 x^{7} y-504 x^{5} y^{3}+504 x^{3} y^{5}-72 x y^{7}$

Da $36^{\mathrm{a}}$ à $38^{\mathrm{a}}$ coluna:

$450 x^{8} y-840 x^{6} y^{3}-3780 x^{4} y^{5}+2520 x^{2} y^{7}-110 y^{9}-70 x^{9}+1080 x^{7} y^{2}+1260 x^{5} y^{4}-4200 x^{3} y^{6}+810 x y^{8} \quad 90 x^{8} y-840 x^{6} y^{3}+1260 x^{4} y^{5}-360 x^{2} y^{7}+10 y^{9}$ $-810 x^{8} y+4200 x^{6} y^{3}-1260 x^{4} y^{5}-1080 x^{2} y^{7}+70 y^{9} \quad 110 x^{9}-2520 x^{7} y^{2}+3780 x^{5} y^{4}+840 x^{3} y^{6}-450 x y^{8}-90 x^{8} y+840 x^{6} y^{3}-1260 x^{4} y^{5}+360 x^{2} y^{7}-10 y^{9}$ $90 x^{9}-1800 x^{7} y^{2}+1260 x^{5} y^{4}+2520 x^{3} y^{6}-630 x y^{8} \quad 630 x^{8} y-2520 x^{6} y^{3}-1260 x^{4} y^{5}+1800 x^{2} y^{7}-90 y^{9} \quad 10 x^{9}-360 x^{7} y^{2}+1260 x^{5} y^{4}-840 x^{3} y^{6}+90 x y^{8}$ 
Da $39^{\mathrm{a}}$ à $41^{\mathrm{a}}$ coluna:
$-10 x^{9}+360 x^{7} y^{2}-1260 x^{5} y^{4}+840 x^{3} y^{6}-90 x y^{8}$
$660 x^{9} y-2640 x^{7} y^{3}-5544 x^{5} y^{5}+7920 x^{3} y^{7}-1100 x y^{9}$
$-88 x^{10}+1980 x^{8} y^{2}-9240 x^{4} y^{6}+3960 x^{2} y^{8}-132 y^{10}$ $10 x^{9}-360 x^{7} y^{2}+1260 x^{5} y^{4}-840 x^{3} y^{6}+90 x y^{8}$ $-1100 x^{9} y+7920 x^{7} y^{3}-5544 x^{5} y^{5}-2640 x^{3} y^{7}+660 x y^{9}$ $132 x^{10}-3960 x^{8} y^{2}+9240 x^{6} y^{4}-1980 x^{2} y^{8}+88 y^{10}$ $90 x^{8} y-840 x^{6} y^{3}+1260 x^{4} y^{5}-360 x^{2} y^{7}+10 y^{9} \quad 110 x^{10}-2970 x^{8} y^{2}+4620 x^{6} y^{4}+4620 x^{4} y^{6}-2970 x^{2} y^{8}+110 y^{10}$ $880 x^{9} y-5280 x^{7} y^{3}+5280 x^{3} y^{7}-880 x y^{9}$

$42^{\mathrm{a}}$ e $43^{\mathrm{a}}$ colunas:

$110 x^{9} y-1320 x^{7} y^{3}+2772 x^{5} y^{5}-1320 x^{3} y^{7}+110 x y^{9} \quad-11 x^{10}+495 x^{8} y^{2}-2310 x^{6} y^{4}+2310 x^{4} y^{6}-495 x^{2} y^{8}+11 y^{10}$ $-110 x^{9} y+1320 x^{7} y^{3}-2772 x^{5} y^{5}+1320 x^{3} y^{7}-110 x y^{9} \quad 11 x^{10}-495 x^{8} y^{2}+2310 x^{6} y^{4}-2310 x^{4} y^{6}+495 x^{2} y^{8}-11 y^{10}$ $11 x^{10}-495 x^{8} y^{2}+2310 x^{6} y^{4}-2310 x^{4} y^{6}+495 x^{2} y^{8}-11 y^{10} \quad 110 x^{9} y-1320 x^{7} y^{3}+2772 x^{5} y^{5}-1320 x^{3} y^{7}+110 x y^{9}$

$44^{\mathrm{a}}$ e $45^{\mathrm{a}}$ colunas:

$924 x^{10} y-5940 x^{8} y^{3}-5544 x^{6} y^{5}+19800 x^{4} y^{7}-5940 x^{2} y^{9}+156 y^{11}-108 x^{11}+3300 x^{9} y^{2}-3960 x^{7} y^{4}-16632 x^{5} y^{6}+13860 x^{3} y^{8}-1452 x y^{10}$ $-1452 x^{10} y+13860 x^{8} y^{3}-16632 x^{6} y^{5}-3960 x^{4} y^{7}+3300 x^{2} y^{9}-108 y^{11} \quad 156 x^{11}-5940 x^{9} y^{2}+19800 x^{7} y^{4}-5544 x^{5} y^{6}-5940 x^{3} y^{8}+924 x y^{10}$ $132 x^{11}-4620 x^{9} y^{2}+11880 x^{7} y^{4}+5544 x^{5} y^{6}-9900 x^{3} y^{8}+1188 x y^{10} \quad 1188 x^{10} y-9900 x^{8} y^{3}+5544 x^{6} y^{5}+11880 x^{4} y^{7}-4620 x^{2} y^{9}+132 y^{11}$

$46^{\mathrm{a}}$ e $47^{\mathrm{a}}$ colunas:

$132 x^{10} y-1980 x^{8} y^{3}+5544 x^{6} y^{5}-3960 x^{4} y^{7}+660 x^{2} y^{9}-12 y^{11}-12 x^{11}+660 x^{9} y^{2}-3960 x^{7} y^{4}+5544 x^{5} y^{6}-1980 x^{3} y^{8}+132 x y^{10}$ $-132 x^{10} y+1980 x^{8} y^{3}-5544 x^{6} y^{5}+3960 x^{4} y^{7}-660 x^{2} y^{9}+12 y^{11} 12 x^{11}-660 x^{9} y^{2}+3960 x^{7} y^{4}-5544 x^{5} y^{6}+1980 x^{3} y^{8}-132 x y^{10}$ $12 x^{11}-660 x^{9} y^{2}+3960 x^{7} y^{4}-5544 x^{5} y^{6}+1980 x^{3} y^{8}-132 x y^{10} \quad 132 x^{10} y-1980 x^{8} y^{3}+5544 x^{6} y^{5}-3960 x^{4} y^{7}+660 x^{2} y^{9}-12 y^{11}$ 


\section{Anexo B - Dedução do sistema linear final do Método da Partição}

Todo equacionamento que segue foi retirado de Alves (2010).

\section{O sistema linear para uma fissura}

Tem-se a seguinte equação que define a condição essencial do Método da Partição, expressando a nulidade das tensões ao longo da linha da fissura "i":

$$
\int_{0}^{a_{i}} \sum_{j=1}^{J}\left[b_{i, j}-\alpha_{j+(i-1) J}+\sum_{k=1}^{M} \alpha_{k} \cdot c_{i, j}^{(k)}\right] Q_{j}\left(\xi_{i} / a_{i}\right) \cdot Q_{j 2}\left(\xi_{i} / a_{i}\right) d \xi=0
$$

com $j, j 2=1, \ldots, J$

Considerando-se apenas uma única fissura $(j 2=1)$ e $n$ termos de aproximação $(J=n)$ tem-se que $\mathrm{M}=\mathrm{I}$ ( $\mathrm{n}^{\circ}$ de fissuras) $\mathrm{J}\left(\mathrm{n}^{\mathrm{o}}\right.$ de termos da aproximação $)=1$ x $n=n$.

Como só há uma fissura, o termo referente à iteração de fissuras se anula:

$$
\sum_{k=1}^{M} \alpha_{k} \cdot c_{i, j}^{(k)} \cdot Q_{j}\left(\xi_{i} / a_{i}\right)=0
$$

Portanto, a Equação (B.1) fica representada por:

$$
\int_{0}^{a_{1}} \sum_{j=1}^{n}\left[b_{1, j}-\alpha_{j+(1-1) n}\right] Q_{j}\left(\xi_{1} / a_{1}\right) \cdot Q_{j 2}\left(\xi_{1} / a_{1}\right) d \xi=0
$$

com $j=1, \ldots, n$ e $j 2=1$.

Desenvolvendo a Equação (B.3), obtêm-se o seguinte sistema linear:

$$
[I G] \cdot\{\alpha\}=\{r\}
$$


Ou seja:

$$
\begin{gathered}
{\left[\begin{array}{cccc}
\int_{0}^{a_{1}}-Q_{1} Q_{1} \partial \xi & \int_{0}^{a_{1}}-Q_{2} Q_{1} \partial \xi & \ldots & \int_{0}^{a_{1}}-Q_{n} Q_{1} \partial \xi \\
\int_{0}^{a_{1}}-Q_{1} Q_{2} \partial \xi & \int_{0}^{a_{1}}-Q_{2} Q_{2} \partial \xi & \ldots & \int_{0}^{a_{1}}-Q_{n} Q_{2} \partial \xi \\
\vdots & \vdots & \ddots & \vdots \\
\int_{0}^{a_{1}}-Q_{1} Q_{n} \partial \xi & \int_{0}^{a_{1}}-Q_{2} Q_{n} \partial \xi & \ldots & \int_{0}^{a_{1}}-Q_{n} Q_{n} \partial \xi
\end{array}\right] \cdot\left[\begin{array}{c}
\alpha_{1} \\
\alpha_{2} \\
\vdots \\
\alpha_{n}
\end{array}\right]} \\
=\left[\begin{array}{c}
-\int_{0}^{a_{1}}\left(b_{1,1} Q_{1} Q_{1}+b_{1,2} Q_{2} Q_{1}+\ldots+b_{1, n} Q_{n} Q_{1}\right) \partial \xi \\
-\int_{0}^{a_{1}}\left(b_{1,1} Q_{1} Q_{2}+b_{1,2} Q_{2} Q_{2}+\ldots+b_{1, n} Q_{n} Q_{2}\right) \partial \xi \\
\vdots \\
-\int_{0}^{a_{1}}\left(b_{1,1} Q_{1} Q_{n}+b_{1,2} Q_{2} Q_{n}+\ldots+b_{1, n} Q_{n} Q_{n}\right) \partial \xi
\end{array}\right]
\end{gathered}
$$

Lembrando que este sistema é utilizado somente para a determinação do FIT para problemas contendo uma única fissura, porém aproximado por $J$ termos.

\section{O sistema linear para várias fissuras}

Para o desenvolvimento do sistema linear, consideram-se os seguintes dados:

- Número de termos da aproximação adotados é igual a "m" ( $J=\mathrm{m})$;

- Número de fissuras é igual a "n" (I=n e $j 2=n)$;

portanto $M=J . I=m . n$.

Definidos os dados do problema, considera-se então a equação do Método da Partição que define a condição de nulidade das tensões na linha da fissura "i": 


$$
\int_{0}^{a_{i}} \sum_{j=1}^{J}\left[b_{i, j}-\alpha_{j+(i-1) J}+\sum_{k=1}^{M} \alpha_{k} \cdot c_{i, j}^{(k)}\right] Q_{j}\left(\xi_{i} / a_{i}\right) \cdot Q_{j 2}\left(\xi_{i} / a_{i}\right) d \xi=0
$$

$\operatorname{com} j, j 2=1, \ldots, J$.

Para $n$ fissuras, temos então o seguinte conjunto de equações:

$$
\begin{aligned}
& \int_{0}^{a_{1}} \sum_{j=1}^{J}\left[b_{1, j}-\alpha_{j}+\sum_{k=1}^{M} \alpha_{k} \cdot c_{1, j}^{(k)}\right] Q_{j}\left(\xi_{1} / a_{1}\right) \cdot Q_{j 2}\left(\xi_{1} / a_{1}\right) d \xi=0 \\
& \int_{0}^{a_{2}} \sum_{j=1}^{J}\left[b_{2, j}-\alpha_{j+J}+\sum_{k=1}^{M} \alpha_{k} \cdot c_{2, j}^{(k)}\right] Q_{j}\left(\xi_{2} / a_{2}\right) \cdot Q_{j 2}\left(\xi_{2} / a_{2}\right) d \xi=0 \\
& \vdots \\
& \int_{0}^{a_{n}} \sum_{j=1}^{J}\left[b_{n, j}-\alpha_{j+(n-1) J}+\sum_{k=1}^{M} \alpha_{k} \cdot c_{n, j}^{(k)}\right] Q_{j}\left(\xi_{n} / a_{n}\right) \cdot Q_{j 2}\left(\xi_{n} / a_{n}\right) d \xi=0
\end{aligned}
$$

com $j, j 2=1, \ldots, J$.

Tomando por referência a primeira equação do conjunto de Equações (B.7), observa-se que esta se desdobra em um novo conjunto de equações, sendo o número de equações deste novo conjunto igual ao número de termos da aproximação $(j 2=1, \ldots, J$ com $J=1, \ldots, m)$ :

$$
\begin{aligned}
& \int_{0}^{a_{1}} \sum_{j=1}^{J}\left[b_{1, j}-\alpha_{j}+\sum_{k=1}^{M} \alpha_{k} \cdot c_{1, j}^{(k)}\right] Q_{j}\left(\xi_{1} / a_{1}\right) \cdot Q_{1}\left(\xi_{1} / a_{1}\right) d \xi=0 \\
& \int_{0}^{a_{1}} \sum_{j=1}^{J}\left[b_{1, j}-\alpha_{j}+\sum_{k=1}^{M} \alpha_{k} \cdot c_{1, j}^{(k)}\right] Q_{j}\left(\xi_{1} / a_{1}\right) \cdot Q_{2}\left(\xi_{1} / a_{1}\right) d \xi=0 \\
& \vdots \\
& \int_{0}^{a_{1}} \sum_{j=1}^{J}\left[b_{1, j}-\alpha_{j}+\sum_{k=1}^{M} \alpha_{k} \cdot c_{1, j}^{(k)}\right] Q_{j}\left(\xi_{1} / a_{1}\right) \cdot Q_{m}\left(\xi_{1} / a_{1}\right) d \xi=0
\end{aligned}
$$

$\operatorname{com} j=1, \ldots, J$.

Sendo assim, desenvolvendo todas as equações do conjunto acima para cada fissura, chega-se ao seguinte sistema linear:

$$
[I G] \cdot\{\alpha\}=\{r\}
$$

onde a matriz $[I G]$ pode ser decomposta em duas: 


$$
[I G]=[I G]_{L}+[I G]_{G}
$$

sendo que $[I G]_{L}$ é uma matriz que possui as integrais responsáveis pelas tensões devido à $P_{L}^{(k)}$ e $[I G]_{G}$ possui as integrais responsáveis pelo efeito de interação entre as fissuras.

A matriz $[I G]_{L} M \times M$ é dada da seguinte forma:

$$
[I G]_{L}=\left[\begin{array}{cccc}
{\left[Q^{(1)}\right]} & {[0]} & \cdots & {[0]} \\
{[0]} & {\left[Q^{(2)}\right]} & \cdots & {[0]} \\
\vdots & \vdots & \ddots & \vdots \\
{[0]} & {[0]} & \cdots & {\left[Q^{(n)}\right]}
\end{array}\right]_{M x M}
$$

onde [0] são sub-matrizes $m \times m$ nulas e $\left[Q^{(i)}\right]$ são sub-matrizes $m \times m$ para $i=1, \ldots, n$, representadas por:

$$
\left[Q^{(i)}\right]=-\left[\begin{array}{cccc}
\int_{0}^{a_{i}} Q_{1} Q_{1} \partial \xi & \int_{0}^{a_{i}} Q_{2} Q_{1} \partial \xi & \cdots & \int_{0}^{a_{i}} Q_{m} Q_{1} \partial \xi \\
\int_{0}^{a_{i}} Q_{1} Q_{2} \partial \xi & \int_{0}^{a_{i}} Q_{2} Q_{2} \partial \xi & \cdots & \int_{0}^{a_{i}} Q_{m} Q_{2} \partial \xi \\
\vdots & \vdots & \ddots & \vdots \\
\int_{0}^{a_{i}} Q_{1} Q_{m} \partial \xi & \int_{0}^{a_{i}} Q_{2} Q_{m} \partial \xi & \cdots & \int_{0}^{a_{i}} Q_{m} Q_{m} \partial \xi
\end{array}\right]
$$

Por outro lado, $[I G]_{G}$ é dada da seguinte forma:

$$
[I G]_{G}=\left[\begin{array}{cccc}
{\left[c Q_{1}^{(1)}\right]} & {\left[c Q_{2}^{(1)}\right]} & \cdots & {\left[c Q_{m}^{(1)}\right]} \\
{\left[c Q_{1}^{(2)}\right]} & {\left[c Q_{2}^{(2)}\right]} & \cdots & {\left[c Q_{m}^{(2)}\right]} \\
\vdots & \vdots & \ddots & \vdots \\
{\left[c Q_{1}^{(n)}\right]} & {\left[c Q_{2}^{(n)}\right]} & \cdots & {\left[c Q_{m}^{(n)}\right]}
\end{array}\right]_{M \times M}
$$

onde $\left[c Q_{j}^{(i)}\right]$ são sub-matrizes $m \times m$ para $i=1, \ldots, n$ e $j=1, \ldots, m$, representadas por: 


$$
\begin{aligned}
& {\left[c Q_{j}^{(i)}\right]=-\left[\begin{array}{ccc}
\int_{0}^{a_{i}} c_{i, 1}^{(1+s)} Q_{1} Q_{1}+\ldots+c_{i, m}^{(1+s)} Q_{m} Q_{1} \partial \xi & \int_{0}^{a_{i}} c_{i, 1}^{(2+s)} Q_{1} Q_{1}+\ldots+c_{i, m}^{(2+s)} Q_{m} Q_{1} \partial \xi & \ldots \\
\int_{0}^{a_{i}} c_{i, 1}^{(1+s)} Q_{1} Q_{2}+\ldots+c_{i, m}^{(1+s)} Q_{m} Q_{2} \partial \xi & \int_{0}^{a_{i}} c_{i, 1}^{(2+s)} Q_{1} Q_{2}+\ldots+c_{i, m}^{(2+s)} Q_{m} Q_{2} \partial \xi & \ldots \\
\vdots & \vdots & \ddots \\
\int_{0}^{a_{i}} c_{i, 1}^{(1+s)} Q_{1} Q_{m}+\ldots+c_{i, m}^{(1+s)} Q_{m} Q_{m} \partial \xi & \int_{0}^{a_{i}} c_{i, 1}^{(2+s)} Q_{1} Q_{m}+\ldots+c_{i, m}^{(2+s)} Q_{m} Q_{m} \partial \xi & \ldots
\end{array}\right.} \\
& \left.\int_{0}^{a_{i}} c_{i, 1}^{(m+s)} Q_{1} Q_{1}+\ldots+c_{i, m}^{(m+s)} Q_{m} Q_{1} \partial \xi\right] \\
& \int_{0}^{a_{i}} c_{i, 1}^{(m+s)} Q_{1} Q_{2}+\ldots+c_{i, m}^{(m+s)} Q_{m} Q_{2} \partial \xi \\
& \int_{0}^{a_{i}} c_{i, 1}^{(m+s)} Q_{1} Q_{m}+\ldots+c_{i, m}^{(m+s)} Q_{m} Q_{m} \partial \xi
\end{aligned}
$$

$\operatorname{com} s=(j-1) m$.

Importante ressaltar que o objetivo da matriz $[I G]_{G}$ é realizar a interação entre as fissuras. Sendo assim, os termos da diagonal principal desta matriz devem ser eliminados, uma vez que não tem sentido algum considerar-se o efeito da fissura sobre ela mesma, o que acarretaria duplicidade de efeitos. Uma outra opção para a não consideração deste efeito é adicionar o somatório abaixo na equação (B.6):

$$
\int_{0}^{a_{i}}-\sum_{j=1}^{J}\left[\sum_{l=1}^{J} \alpha_{l+(i-1) J} \cdot c_{i, j}^{[l+(i-1) J]} \cdot Q_{j}\right] Q_{j 2} \partial \xi
$$

$\operatorname{com} j, j 2=1, \ldots, J$.

Definida então a matriz $[I G]$ resta-se destacar o vetor-solução $\{\alpha\}$ :

$$
\{\alpha\}=\left\{\begin{array}{c}
\alpha_{1} \\
\alpha_{2} \\
\vdots \\
\alpha_{M}
\end{array}\right\}
$$


assim como o vetor $\{r\}$, responsável pelas integrais das tensões em $P_{G}^{(0)}$, apresentado da seguinte maneira:

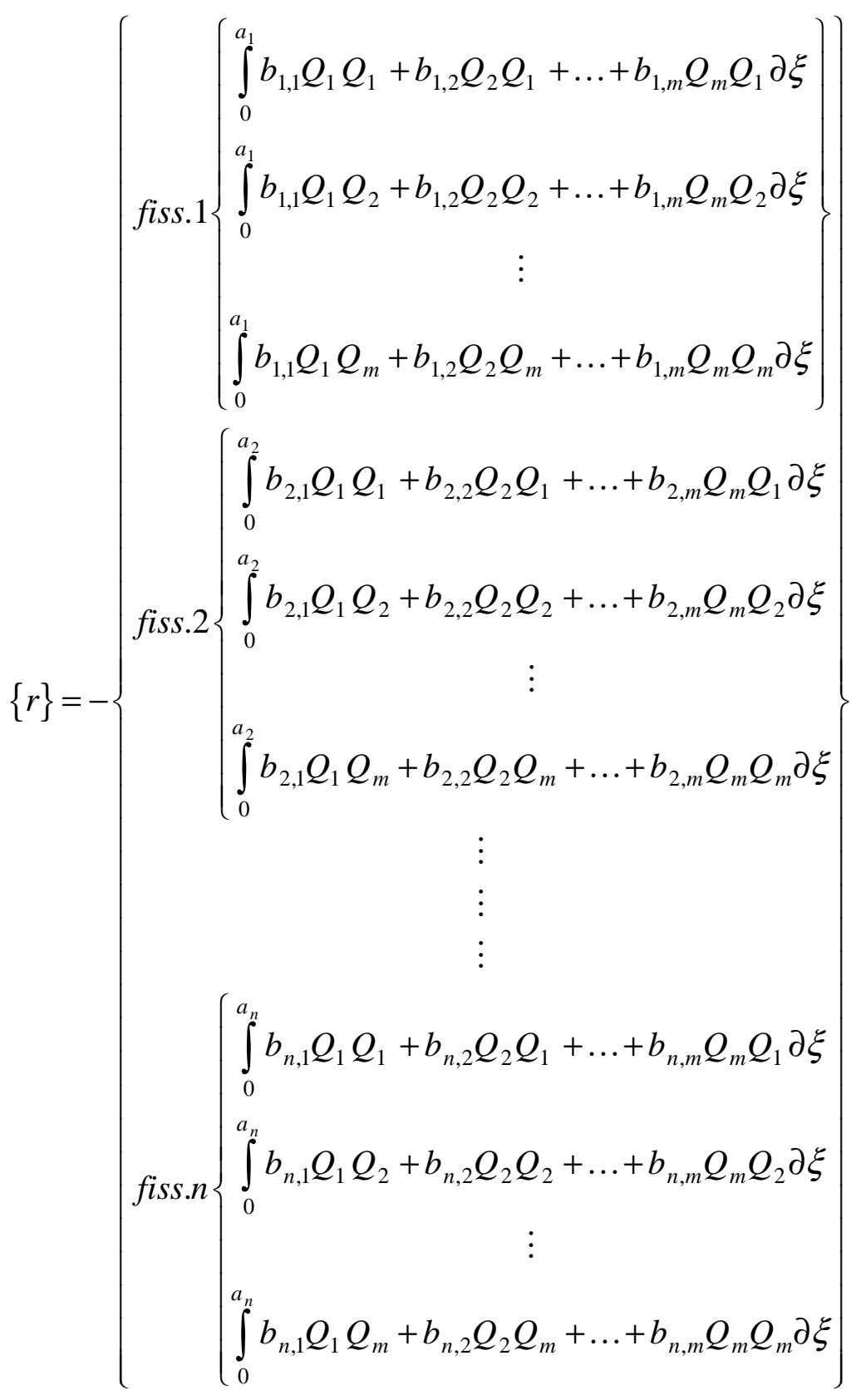

